\title{
Proliferation Risk Characterization Model Prototype Model-User and Programmer Guidelines
}

\author{
J. S. Dukelow \\ D. Whitford
}

December 1998

Prepared for

the U.S. Department of Energy

under Contract DE-AC0.6-76RLO 1830

Pacific Northwest National Laboratory

Richland, Washington 99352 


\section{DISCLAIMER}

This report was prepared as an account of work sponsored by an agency of the United States Government. Neither the United States Government nor any agency thereof, nor any of their employees, make any warranty, express or implied, or assumes any legal liability or responsibility for the accuracy, completeness, or usefulness of any information, apparatus, product, or process disclosed, or represents that its use would not infringe privately owned rights. Reference herein to any specific commercial product, process, or service by trade name, trademark, manufacturer, or otherwise does not necessarily constitute or imply its endorsement, recommendation, or favoring by the United States Government or any agency thereof. The views and opinions of authors expressed herein do not necessarily state or reflect those of the United States Government or any agency thereof. 


\section{DISCLAIMER}

Portions of this document may be illegible in electronic image products. Images are produced from the best available original document. 


\section{Summary}

This report documents completion of a methodology for characterizing nuclear material proliferation risk. The research was conducted for the Department of Energy, Office of Intelligence (DOE/NN) under the Fiscal Year 1997 Nuclear Energy and Material program guidance.

The project required development of a risk model and application of the resultant model to several real-world scenarios. Results were used to validate the approach and make program management decisions. The model was developed and refined through a number of scenarios during 1997 and 1998 using single-user and group-analysis situations; it proved to be a valuable tool for comparative risk analysis.

The U.S. Department of Energy (DOE), Office of Intelligence, identified the need for a tool for collecting information about the risks of diversion of weapons-capable material that would capture information from many sources: open literature, intelligence sources, and the judgment of experts. The tool should provide a method for comparing judgments about particular threat and site vulnerability, building a foundation for a consistent and comparable judgment about vulnerability and threat combinations-ultimately leading to assessment of proliferation risk.

A model for estimating the risk of diversion of weapons-capable materials was developed. The model represents both the THREAT of diversion and site VULNERABILITY as a product of a small number of variables, each of which can take on a small number of qualitatively defined (but quantitatively implemented) values. The values of the overall threat and vulnerability variables are computed as products of the sub-variables, which are then converted to threat and vulnerability categories. The threat and vulnerability categories are used to define the LIKELIHOOD of diversion, also defined categorically. The evaluator supplies an estimate of the CONSEQUENCES of a diversion, defined categorically, but with the categories based on the International Atomic Energy Agency (IAEA) Attractiveness levels.

LIKELIHOOD and CONSEQUENCES categories are used to define the RISK, also defined categorically. The threat, vulnerability, and consequences inputs provided by the evaluator contain a representation of uncertainty in each variable assignment, which is propagated all the way through to the calculation of the Risk categories.

The resultant model was tested in several realistic scenarios and was subsequently used to assess relative risk for program options. The results were satisfactory and supported user needs. The model stimulated interaction when used with multiple participants, and allowed identification of information voids and divergent views. Intelligence shortfalls were readily apparent in use of the model. 
Though analytically sound and viable, the routine application of the model for site characterization was time consuming and required expertise beyond the capacity of a single analyst. The best value in use of the model was found in group-interaction with a focus on assessment of particular scenarios requiring risk quantification.

Nominal case studies replace classified material for this report. Multiple files are contained in Appendix G. These files contain executable code for the scenarios discussed in the report, a summary of the project, and worksheets for the scenarios. 


\title{
Acknowledgments and Project Participants
}

\author{
Development of Conceptual Model \\ David Seaver \\ William A. Hesser \\ GariAnne Gelston \\ Rudy Hanson \\ James Dukelow
}

Model Implementation and Review

Richard Hanlen

Charles Willingham

Keri Robinson

John Loftis

James Dukelow

Dan Whitford

Richard Gilbert 


\section{Contents}

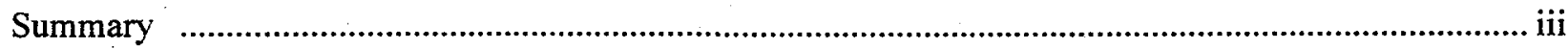

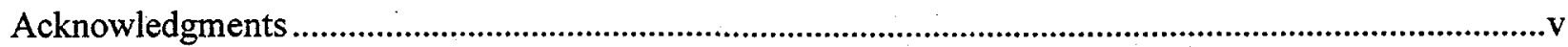

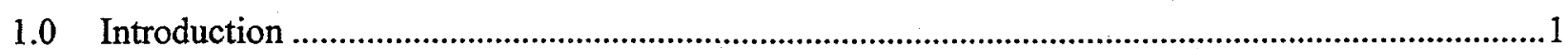

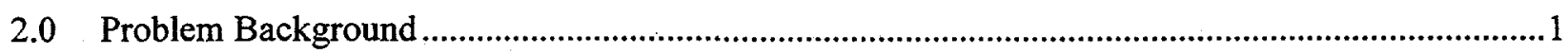

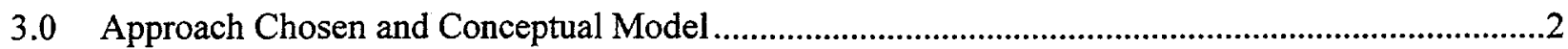

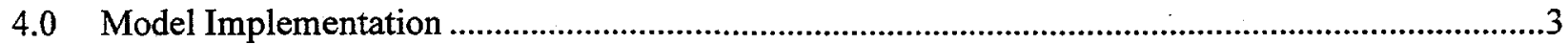

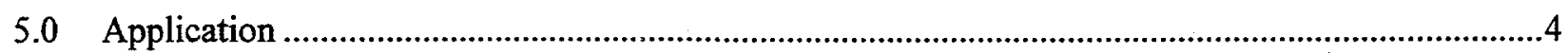

6.0 Strengths, Weaknesses, and Suggestions for Further Development ........................................6

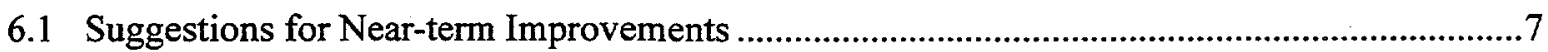

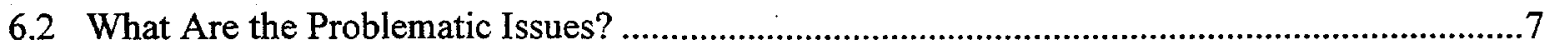

6.3 Longer Term Fixes and Modifications to the Evaluation Program .......................................

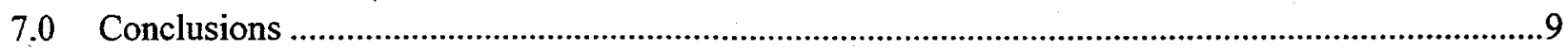

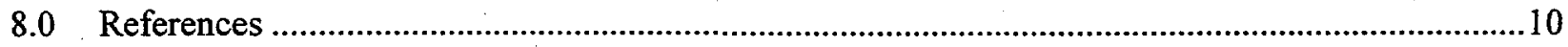

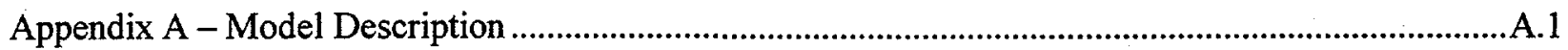

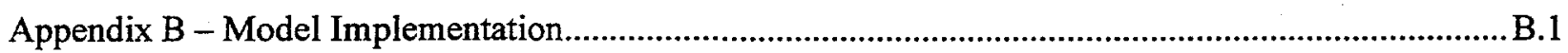

Appendix C - Basic Concepts of Probability Theory …........................................................................

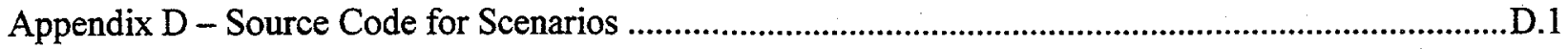

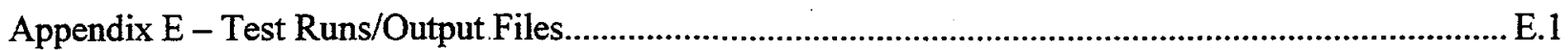

Appendix F - Sample Calculation for a Simplified Model .............................................................. F.1

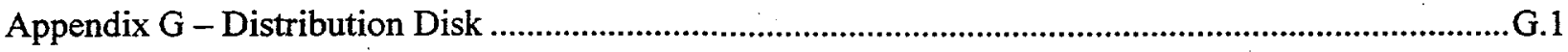




\subsection{Introduction}

The U.S. Department of Energy (DOE), Office of Intelligence, identified the need for a tool for collecting information about the risks of diversion of weapons-capable material that would capture information from many sources: open literature, intelligence sources, and the judgment of experts. The tool should provide a method for comparing the judgments of different experts about a particular threat and site vulnerability and a method for a single expert to express internally consistent and comparable judgments about a number of different site vulnerability and threat combinations.

The main focus of this effort was the estimation of risk of diversion of fissile and other nuclear weapons-capable materials from sites in the former Soviet Union. However, the conceptual model for the tool developed should be equally applicable to diversion of chemical and biological warfare materials.

Section 2.0 describes some earlier work on this problem. Section 3.0 gives the approach chosen and provides a general description of the conceptual model used. Appendix A provides a more detailed description of the conceptual model. Section 4.0 gives a general description of implementation of the conceptual model, with more detail provided in Appendix B. Section 5.0 describes a real-world test of the model. Section 6.0 gives an assessment of strengths and weaknesses of the approach chosen and its implementation, and gives some suggestions for further development of the model. Appendix C describes the concepts from probability used to incorporated propagation of uncertainties into the implementation of the model. Appendix $D$ gives the source code for the five programs that implement the model for the five generic diversion scenarios. Appendix E gives the test output from three generic test sets used in tuning the models for each of the five diversion scenarios (fifteen output files in all). Appendix $\mathrm{F}$ is a sample calculation of a simplified model of the program intended to help the reader understand the operation of the program. Appendix $\mathrm{G}$ contains electronic files containing analyst worksheets, executable code for the scenarios, and an overview of the project.

\subsection{Problem Background}

Some earlier efforts to estimate risks were considered during the conceptual design of this model. One attempt to estimate risk of weapons-material diversions used a questionnaire of approximately ninety questions, to be answered "Yes" or "No" and associated a risk estimate with the number of "Yes" answers to the questions. Users of the questionnaire were unwilling to answer many of the questions, because of uncertainty or lack of knowledge, leading to difficulty in interpreting the results of the questionnaire.

A white paper, A Procedure for Risk Ranking for Federal Risk Management Agencies (Morgan et al. 1994), describes two distinct types of risk estimation models. The first, the holistic model, uses general

discussion of risk characteristics and stakeholder values, followed by a process of ranking and/or binning 
the entities (waste sites, technologies, policy choices, etc.) all at once and then dividing them into a few general classes (most rank high, most rank medium, most rank low, ambiguous ranking), based on the aggregation of the rankings of different risk estimators.

The second type of risk estimation model is the attribute-weighting procedure. For each entity being evaluated, numerical values are assigned by risk estimators to each of a set of risk attributes. Finally, the risk score for the entity is calculated as a weighted average of the assigned values and the risk ranking or prioritization is based on the weighted averages.

The model we are proposing is an attribute-weighting model, with the additional capability of builtin uncertainty propagation. A somewhat similar attribute-weighting model is described by Dreiser and Rutherford (1996). The Dreiser and Rutherford paper was reviewed by the PNNL team that developed the conceptual model described in this report. Dreiser and Rutherford used an additive measure of risk and did not incorporate a scheme for handling uncertainties.

\subsection{Approach Chosen and Conceptual Model}

Our model attempts to provide a robust and stable method that experts in the areas of threat and vulnerability assessment can use to estimate risks associated with attempts to divert weapons or weapons-capable materials from research, fabrication, and storage sites around the world. By robust and stable, we mean that estimates by the same experts can be compared across sites, and, perhaps with greater difficulty, estimates by different experts of the risk for a particular site can be compared and the basis for any differences analyzed.

We were asked to develop risk estimation models for four generic risk diversion scenarios. Somewhat later, we were asked to develop a model for diversion of material during transportation.

- The Insider Theft scenario involves theft or diversion by one or a small number of facility insiders, perhaps over an extended period of time.

- The Outsider Theft scenario involves a covert attack on the facility by one or a small group of outsiders, perhaps with insider collusion.

- The Outsider Attack scenario involves an overt attack on a facility by a small to large outsider attack group, perhaps with insider collusion.

- The State Transfer scenario involves the decision by all or part of the host government to transfer material out of the host country in violation of non-proliferation agreements.

- The Transportation Diversion scenario involves an overt or covert attack on material while moving from a host country facility to another facility inside or outside the host country. 
The current model defines the threat and vulnerability measures as products of a few variables that are approximately independent of each other. These products are converted to threat and vulnerability categories (Negligible, Low, High, and Very High). Each combination of a threat category and a vulnerability category corresponds to one of five Likelihood categories (Negligible, Low, Medium, High, and Very High, qualitatively describing the likelihood of a successful diversion). The evaluator is asked to estimate the consequences of a successful diversion, also defined qualitatively as one of five categories (but with the qualitative categories defined in terms of the IAEA Attractiveness Levels). Finally, each combination of a Likelihood category and a Consequence category corresponds to one of five Risk categories. The Consequence and Risk measures use the same categories as the Likelihood measure. At each stage of the calculation, the evaluator's uncertainties in estimates of threats, vulnerabilities, and consequences are propagated through the calculation to the final definition of the Risk categories.

Details of the conceptual model are given in Appendix A.

\subsection{Model Implementation}

This model has been implemented in five computer programs, one for each of five different diversion scenario types. For each scenario type considered and for a particular site/facility and threat combination, the program solicits input from evaluators and calculates an aggregate threat measure and an aggregate vulnerability measure for the combination of a particular site/facility and some threat group (of the collection of threat groups). The Threat and Vulnerability measures are both products of sets of submeasures. The likelihood of a successful diversion is then calculated as a function of the threat and vulnerabilities. An estimate of the consequences of a diversion is then solicited from the evaluator and the associated risk from diversion is calculated as a function of the Likelihood and Consequence measures. This relationship between threat, vulnerability, likelihood, consequences, and risk is consistent with current practice in risk analysis.

An important feature of the model is that it explicitly incorporates information about the expert evaluator's estimate uncertainties. At each stage, the program requires that the evaluators estimate their uncertainty about the measures used and propagates those uncertainties through the risk calculation using standard techniques from probability theory for manipulating random variables.

There are two possible sources for evaluator uncertainty. The evaluator might not have enough information about a particular site and threat group to make a confident estimate for a particular variable. The other possibility is that there is some essential uncertainty about the value to be assigned to a particular variable. An example of the second source of uncertainty would be the evaluation of the vulnerability sub-variable Y6, Access Controls, in the situation where a site has excellent access controls installed, but they are only manned part of the time on a schedule that wasn't accessible to the intelligence capability of the threat group.

Details of implementation of the conceptual model are given in Appendix B. 


\subsection{Application}

This project required application and testing of the model using "real world" scenarios and comparison with risk determinations developed by other techniques. The results were comparable and provided insights not available using other techniques. Particular value was found in critical examination of absolute results, examination of why those results were obtained, and further examination of the model for variable sensitivities and reasons for outcome variance.

Additional opportunities to apply the model emerged during development and after completion of the model presented in this paper. The model was applied to multiple scenarios and analysts critiqued the results. Classification constraints preclude full discussion of the specific scenarios. Several examples are presented in Appendix $\mathrm{G}$ to demonstrate model use, along with nominal worksheets to support data entry.

During the developmental work and practical applications, the investigators found that success was predicated on preliminary work prior to applying the models. We found that it was necessary to ensure that

- the models were sufficient to consider the problem being addressed

- the participants agreed the model was analytically adequate (or agreed to provide inputs without fully understanding the model details)

- the inputs were relatively free of particular biases - e.g., proprietary, personal, or business interests

- the results could be conveyed in terms relevant to the end-user.

Several potential means for applying the model evolved and were used in developmental and practical applications:

- single user

- group user.

We found that the broad range of information required to adequately analyze the scenarios addressed by the model were beyond the range of most individual users. As discussed elsewhere, full use of the model requires expertise in political, economic, and technical fields. Few users had the range of expertise to exploit the model. Individual users examined a large number of scenarios. In many cases, default answers were apparent because the users did not have conclusive informed opinions that allowed assigning definitive values. Conclusion: this model is not a good technique for individual users.

The "Group-user" technique proved more productive. Several variations were examined. As the model depends on a single value for each of the variable determinants, the issue became how to obtain a 
single value (or set of values), how to combine them, and how to examine the output of a single or several runs for the model. This, in turn, evolved into an expanded effort related to decision theory and group dynamics that exceeds the task scope. In summary, several techniques proved adequate:

- averaging inputs

- weighting inputs

- interactive examination.

Examining each of these areas separately, we find that

- averaging inputs (the variables) has the inherent flaw of diluting the most representative (and possibly correct) inputs.

- prescribed weighting of inputs requires assessment of each contributors' value and puts a burden on the analyst assessing use of the model.

- interactive examination requires complete examination of group dynamics, an analytically concise construct of the evaluation process, and a set of rules for formulating use of the model.

An unexpected problem was encountered in representing model outputs. As designed, the model provides a probability (a numerical value) associated with each of categories of risk ranging from Negligible to Very High. While this presentation was useful, an absolute numerical representation of risk (without regard to risk category) proved more useful in comparing various scenarios or iterative runs of the same scenario. In order to generate an absolute number, weighted values of the probability of risk in each category were developed. For example, in Table 1, a weighted value for risk was determined as 3.3, on a scale of 1 to 5. For this scenario, risk would be considered as Medium to High.

In summary, the best outcome was achieved in the "Group-user" use of the model. Early definition of the allowed group-dynamics and "ground-rules" for use of the model facilitated the process. Presentation of results was refined in conjunction with tests and application to practical scenarios. Overall, the model proved adequate for "real-world" use. 
Table 1. Risk Range Calculation. Each category of risk is assigned a numerical weighting factor. The expert fills in (italics) the estimated probability of the risk falling into each category. These probabilities must total 1.0. The probabilities are multiplied by their respective weighing factors to give a weighted value for each category. The sum of the weighted values is equated to a qualitative risk by using the categories and weighing factors. In this example, the 3.3 total weighted value equates to a high to medium risk.

\begin{tabular}{|l|c|c|c|l|}
\hline Category & Weighting Factor & Probability & Weighted Value & \multicolumn{1}{|c|}{ Risk Range } \\
\hline Very High & 5 & 1 & 5 & High-to-Medium Risk \\
\cline { 1 - 4 } High & 4 & 3 & 1.2 & \\
\hline Medium & 3 & 4 & 1.2 & \\
\hline Low & 2 & 2 & 4 & \\
\hline Negligible & 1 & 0 & 0 & \\
\hline Total & & 1.0 & 3.3 & \\
\hline
\end{tabular}

\subsection{Strengths, Weaknesses, and Suggestions for Further Development}

The proposed model, when properly tuned or calibrated by comparing its output with the explicit knowledge and intuition of the best experts on these threat and vulnerability issues, may provide a mechanism for leveraging that knowledge into a tool that can be used by a wide variety of personnel. Since the calculations are explicit, an audit trail can be produced that provides evaluators with a basis for discussing in detail their differing assessments of diversion risks. The input required of the expert evaluators is relatively simple, consisting of assigning probabilities or weights to the two to four values of each of several (two to eight) threat and vulnerability sub-measures. In addition, the evaluator is asked to provide probabilities or weights for each of the five consequence categories (which are defined using the IAEA classification of quantities and types of weapons-usable materials). The uncertaintyhandling scheme provides a measure of the impact of evaluator uncertainty about the input concerning the diversion risk.

Because of the tuning/calibration process required for the model to produce reasonable results (that is, results consistent with the knowledge and intuition of experts), there is subjectivity in the model that is buried in the implementation details. We consider this to be unavoidable because there aren't any "first principles" in the sense of physical or chemical laws that would permit a first-principles model of diversion risk.

Another major implementation detail that requires further validation is the assumption that the threat and vulnerability sub-measures and the threat, vulnerability; and consequence categorical random variables are all probabilistically independent. This assumption implies that their probabilities can be computed using the usual product-of-probabilities rule for independent events. Although, this assumption is 
arguable, we consider it defensible. Not assuming independence would require the evaluators to provide estimates not only of the values of the threat and vulnerability sub-measures and the consequence categories, but also estimates of how all of those variables were correlated with each other. That would greatly expand the amount of input the evaluators had to provide. In addition, estimating the correlation or dependency between variables seems like a much more difficult task than simply estimating values of the variables and our uncertainty of our knowledge of those variables.

Although, the threat and vulnerability sub-measures were chosen to be more or less independent of each other, we recognize that there is, in fact, some dependency between them. Part of the effort in the model tuning and calibration process was in modifying the structure of particular sub-measures and how the threat and vulnerability variables were computed from the sub-measure values, for the purpose of minimizing the impact of residual correlation or dependency between the variables.

\subsection{Suggestions for Near-term Improvements}

- Fix the handling of single- and double-precision variables and the output print formatting so that no meaningless output (for example, the last eight to nine digits of some of the output variables) is presented.

- Enable input from a file so that a user won't have to reenter the same input multiple times.

- Provide a simple graphical representation of the histogram output.

- Print the threat and vulnerability category probabilities to the output file.

- Modify input solicitation screens to eliminate clutter.

\subsection{What Are the Problematic Issues?}

The particular choices of threat and vulnerability sub-measures and the consequence categories are derived from the conceptual model development done in July and August 1997 by a team of PNNL personnel. They are not set in stone, however. We may want to delete or modify some of the sub-measures or add new ones as the program is used/tested by subject matter experts. A major review and vetting exercise by project staff in November 1997 resulted in several changes to threat and vulnerability sub-measures and to the likelihood and risk matrices.

The major potential skeleton in the closet of the model and the prototype evaluation programs is that some of the threat and vulnerability sub-measures are correlated, but the model and the program treat them as if they are mutually independent. The result is that the probabilities assigned to combinations of sub-measures that correspond to high (or low) overall threat or vulnerability may be either too low or too high depending on the situation. We intentionally chose to treat these as statistically independent 
variables, because the alternative (treating the variables as correlated and using conditional probabilities) would greatly increase the complexity of the models and the amount and complexity of input required from the expert user of the models.

We have used several approaches to mitigate this problem. First, the division points used to convert the threat and vulnerability measure numerical values to categorical values (i.e., negligible, low, etc.) can be placed in the threat/vulnerability measure numerical range in such a way as to partially counteract the effects of correlation of the sub-measures. To some extent this was done when the division points used in the prototype program were established, as can be seen by looking at the table of numerical division points given in Appendix B.

Second, the threat/vulnerability sub-measures can be reworked (which would mainly mean coalescing correlated sub-measures into one or, perhaps, two sub-measures). The goal would be to arrive at a set of sub-measures that were actually mutually independent. Several of the changes resulting from the November review/vetting session were aimed at increasing the statistical independence of the model variables.

Third, also arising from the November 1997 review, for some of the scenario types and some of the vulnerability sub-measures, the product of a couple of the sub-measures is replaced by the maximum of the two measures. This effectively assumes that one of the measures is a direct consequence of the other, i.e., we assume an extreme form of statistical dependence between the two variables. This is done with the pair $Y_{5}$ and $Y_{6}$, and the pair $Y_{7}$ and $Y_{8}$ in Outsider Theft and Outsider Attack.

Finally, the assignment of probabilities to Threat, Vulnerability, and Consequence measure random variables by the expert evaluator implies a definition of "probability" that some students of probability and statistics do not agree with. The assignment may represent a true variability; for instance, we may assign probabilities of

$$
\mathrm{P}\left(\mathrm{Y}_{4}=0.5\right)=0.5, \mathrm{P}\left(\mathrm{Y}_{4}=1.0\right)=0 \text {, and } \mathrm{P}\left(\mathrm{Y}_{4}=2.0\right)=0.5
$$

to the intrusion detection Vulnerability sub-measure. This would mean that half the time intrusion detection was excellent and half the time it was poor, because we had firm information that the overall intrusion detection system was only functional half the time.

We might also assign these same probabilities to $Y_{4}$ if we knew there was an excellent intrusion detection system being implemented at a site, but were unaware whether installation and testing was complete. In this second case, our assignment of probabilities represents our "degree of belief" that a certain state of the world is true or not. Within probability and statistics, those whose believe in "degree of belief" assignments of probability are called subjectivists or "Bayesians" and those who disagree are called classicists, or "frequentists". The implementor of our model is a believer in the Kolmogorov axioms for probability theory and is comfortable with either "degree of belief" assignments or with using 
probabilities to represent true variability. At least one of our internal reviewers is a classicist and prefers to call those assignments "weights", rather than probabilities, but otherwise seems comfortable with the model.

\subsection{Longer-Term Fixes and Modifications to the Evaluation Program}

The current model was intentionally designed as a conceptually simple model that could be quickly implemented and tested. A major reason for this preference for simplicity was the experience with. earlier efforts to build such evaluation models, which required much detailed input from the subject matter experts and lacked any mechanism for dealing with situations where the expert was not comfortable responding to the detailed questions. The present model simplifies the required input and incorporates into the model a mechanism (based on probability theory) for accommodating expert uncertainty about required input.

It would be desirable to improve the user interface of the evaluation program. This might involve implementing it in Visual Basic or some other language for developing programs in a Windows-style GUI. Other possibilities would be to implement it as a spreadsheet or as a Java applet running in the same Web page that has the site/facility database. Spreadsheet implementation would require the user to have access to the same spreadsheet program. If the spreadsheet included uncertainty modeling and propagation, then the user would also have to have access to the uncertainty add-in program (probably (a) Risk or Crystal Ball) used to do that modeling.

Finally, depending on the results of testing of the models by expert users, it may become necessary replace the current treatment of threat and vulnerability sub-measures as statistically independent variables, by a more rigorous treatment using correlation and conditional probabilities. This would introduce a complexity to both the calculations and the information required of the experts that, at this time, doesn't seem desirable, but which may become necessary.

\subsection{Conclusions}

A model for the estimation of the risk of diversion of weapons-capable materials was developed. It represents both the threat of diversion and site vulnerability as a product of a small number of variables (two to eight), each of which can take on a small number (two to four) of qualitatively defined (but quantitatively implemented) values. The values of the overall threat and vulnerability variables are then converted to threat and vulnerability categories. The threat and vulnerability categories are used to define the likelihood of diversion, also defined categorically. The evaluator supplies an estimate of the consequences of a diversion, defined categorically, but with the categories based on the IAEA Attractiveness levels. Likelihood and Consequences categories are used to define the Risk, also defined categorically. The threat, vulnerability, and consequences input provided by the evaluator contains a representation of his/her uncertainty in each variable assignment, which is propagated all the way through to the calculation of the Risk categories. 


\subsection{References}

Dreiser, J. C. and D. Rutherford. 1966. "Fissile Material Proliferation Risk," Journal of Nuclear Materials Management, 24(5):30-35.

Morgan, G., B. Fischhoff, L. Lave, P. Fischbeck, S. Byran, K. Jenni, G. Louis, S. McBride, L. Painton, S. Siegel, and N. Welch. 1994. A Procedure for Risk Ranking for Federal Risk Management Agencies, white paper, Carnegie-Mellon University, Philadelphia, Pennsylvania. 


\section{Appendix A}

\section{Model Description}




\section{APPENDIX A - Model Description}

\section{Details of the Conceptual Model}

The threat and vulnerability sub-measures used in this scheme for estimating the risk of diversion of nuclear materials are all treated as random variables, requiring that probabilities (or weights) summing to 1 must be assigned to each of the numerical values taken on by that sub-measure. The input routines enforce this requirement.

In addition, the assignment of probabilities to the sub-measure can be used to represent the evaluator's uncertainty about the correct value of the sub-measure. For instance, in the absence of any information about the true value of the sub-measure (i.e., complete uncertainty), the evaluator should assign equal weights to all of the sub-measure values.

Threat Sub-measures

Existence of a Long Standing Conflict

$\mathrm{X}_{1}=0.5$, no underlying conflict

$X_{1}=1.0$, recently developed conflict (decades-long duration)

$X_{1}=2.0$, long standing conflict (centuries-long duration)

"Long Standing Conflict" is shorthand for aspects of intensity and long duration in the motivation of the threat groups. It can derive from extreme religious prejudice, language/cultural differences, and/or from a long history of oppression, suppression, or warfare between two groups of people. Examples include Irish Catholics vs. Irish Protestants and the British or the Basques vs. the Spanish, and Islamic fundamentalists vs. secular Moslem governments.

Political/ldeological Motivations

$\mathrm{X}_{2}=0.5$, no political/ideological basis

$X_{2}=1.0$, political/ideological motivations

Examples would be support for terrorism as a component of the cold war struggle between Communism and the West. 


\section{Religious Motivations}

$X_{3}=0.5$, no religious animosity

$X_{3}=1.0$, some religious animosity

$X_{3}=2.0$, strong religious animosity

The intent here is to capture aspects of motivation distinct from possible religious components of the "long-standing conflict" measure above.

The threat sub-variable $X_{3}$ is no longer being used, since we judged that the properties it was measuring were adequately covered by $X_{1}$ and $X_{2}$

\section{Perception of target vulnerability}

$X_{4}=0.5$, facility not perceived as vulnerable

$X_{4}=1.5$, facility perceived as vulnerable

The reasoning here is that, even for a threat group predisposed to action, if a particular facility is perceived as not vulnerable, they will direct their attention elsewhere.

\section{Availability of resources}

$X_{5}=0.0$, no resources available

$X_{5}=0.5$, modest, locally collected resources

$X_{5}=1.0$, intermediate, with outside support

$X_{5}=2.0$, large, state-supported

This is a measure of the resource base of the threat group.

For the Insider Theft case, we modified Subroutine 150 so that $X_{5}$ takes on only the values 0.5 and 1.5 . For simplicity, we simply set the probabilities of the 0.0 and 2.0 values to zero and changed the 1.0 value to 1.5 . The user is then solicited to provide probabilities summing to 1.0 for the 0.5 and 1.5 values of $X 5 \mathrm{~V}$. The rationale for this change is that the insider is never completely without resources and is also not likely to have the resources of a nation state.

\section{Training and leadership}

$\mathrm{X}_{6}=0.5$, poor training and leadership

$X_{6}=1.0$, intermediate training and leadership

$X_{6}=2.0$, well-trained and led

This is a measure of the quality of training and leadership of the threat organization. 
Knowledge and intelligence

$X_{7}=0.5$, poor intelligence capabilities

$X_{7}=1.5$, good intelligence capabilities

This is a measure of the access of the threat organization to information about the potential target and their ability to use that information.

For the Outsider Attack and Outsider Theft models, the larger of the two $X_{7}$ values is set at 2.0, instead of 1.5. The rationale here is that intelligence capabilities are more important to outsiders that to insiders.

Site/facility socio-economic conditions

$X_{8}=0.75$, good conditions

$X_{8}=1.0$, not-so-good

$X_{8}=1.5$, extremely difficult conditions

This attempts to measure the likelihood that the threat organization can subvert/co-opt insiders to support its diversion attempt or that insiders will themselves attempt diversion.

Vulnerability Sub-measures

Material Surveillance

$Y_{1}=0.5$, excellent system of material surveillance

$Y_{1}=1.0$, good system of material surveillance

$Y_{1}=2.0$, poor system of material surveillance

This measures the material accounting and surveillance infrastructure that enables facility management to detect attempted or successful diversion.

\section{Transfer Controls}

$Y_{2}=0.5$, excellent system of transfer control

$Y_{2}=1.0$, good system of transfer control

$Y_{2}=2.0$, poor system of transfer control

This measures the security of material in transit from one storage/utilization location to another (within the same facility). 


\section{Protective Forces}

$Y_{3}=0.5$, excellent protective forces

$Y_{3}=1.0$, good protective forces

$Y_{3}=2.0$, poor protective forces

This is a measure of the ability of the facility guard forces to prevent or delay a diversion.

Intrusion Detection

$Y_{4}=0.5$, excellent intrusion detection

$Y_{4}=1.0$, good intrusion detection

$\mathrm{Y}_{4}=2.0$, poor intrusion detection

This measures infrastructure provisions for the detection of outsider attack or theft attempts.

\section{Barriers/Delays}

$Y_{5}=0.5$, excellent barriers/delays

$Y_{5}=1.0$, good barriers/delays

$Y_{5}=2.0$, poor barriers/delays

This measures facility design and operation features that tend to delay a diversion attempt long enough for response of off-site protection forces.

Access Controls

$Y_{6}=0.5$, excellent access controls

$Y_{6}=1.0$, good access controls

$Y_{6}=2.0$, poor access controls

This measures controls aimed at preventing outsider access to material storage areas and to constraining the ability of insiders to gradually divert materials.

\section{Site Location}

$Y_{7}=0.5$, site with none or few good escape routes

$Y_{7}=1.5$, site with many good escape routes

This measures the ability of group or individual that has successfully diverted material to successfully remove it to a secure location and/or transfer it to the end-user. 


\section{Emergency Response}

$Y_{8}=0.5$, site with excellent emergency response resources

$Y_{8}=1.0$, site with some emergency response

$Y_{8}=2.0$, site with none or poor emergency response

This measures the ability of off-site emergency response to interdict a forcible diversion in progress, given timely notification. Note that measures $Y_{4}, Y_{5}$, and $Y_{7}$ also condition the likelihood of successful interdiction of a diversion in progress.

Overall Capability of Host Country Safeguards Measures

$Y_{9}=0.5$, robust safeguards measures

$Y_{9}=1.0$, safeguards with some weaknesses in design or implementation

$Y_{9}=2.0$, weak or non-existent safeguards

This area measures the overall capability of safeguards measures to detect/alarm attempted state transfer of weapons material to unauthorized state- or sub-state organizations.

\section{Socio-political stability}

$Y_{10}=0.5$, stable government with good foreign relations, checks and balances $Y_{10}=1.0$, some instability, some foreign enemies, some checks and balances $Y_{10}=2.0$, significant instability, many factions, many enemies, no checks and balances

This attempts to measure the likelihood that all or part of the relevant government organizations will formulate and implement or will fail to impede a state transfer diversion attempt.

\section{Location Monitoring and Attack Detection}

$\mathrm{Y}_{11}=0.5$, excellent location monitoring and attack detection

$Y_{11}=1.0$, good location monitoring and attack detection

$Y_{11}=2.0$, poor location monitoring and attack detection

This measures the existence or lack of real-time systems for monitoring the location of the shipment and detecting an attack on the shipment. This information is needed to enable emergency response by protection forces other than those accompanying the shipment. 


\section{Accompanying Protection Forces}

$Y_{12}=0.5$, adequate protection forces accompanying shipment

$Y_{12}=1.0$, some accompanying forces

$Y_{12}=2.0$, no significant accompanying forces

This measures those protection forces accompanying the shipment and available for immediate response to an attack on the shipment.

\section{Use of Deception and Indirection}

$Y_{13}=0.75$, well-executed deception and indirection

$Y_{13}=1.0$, some use of deception and indirection

$Y_{13}=1.5$, no use of deception and indirection

This measures the use of deception and indirection to disguise the time, planned itinerary, and planned protective systems for the shipment.

\section{Immobilization and Delay}

$Y_{14}=0.5$, excellent systems for delaying the attackers and immobilizing the nuclear materials

$Y_{14}=1.0$, some systems for delaying the attackers and immobilizing the nuclear materials

$Y_{14}=1.25$, no systems for delaying the attackers and immobilizing the nuclear materials

This measures the systems for delaying the progress of an attack on the shipment or for physically immobilizing the nuclear materials. Good immobilization can thwart an attack. Delays provide more time for the response of non-accompanying protection forces.

\section{Attack Sites and Escape Routes}

$Y_{15}=0.5$, few attack sites and few escape routes

$Y_{15}=1.0$, intermediate mixture of attack sites and escape routes

$Y_{15}=2.0$, many attack sites and many escape routes

This measures the ease with which a shipment can be attacked and the variety of escape routes available after a successful attack. 


\section{Non-accompanying Emergency Response Forces}

$Y_{16}=0.5$, excellent array of protection forces available to respond to an attack in progress

$Y_{16}=1.0$, some protection forces available to respond to respond to an attack in progress

$Y_{16}=2.0$, no significant protection forces available to respond to an attack in progress

This measures the additional forces that can be brought to bear upon an attack in progress or used to interdict the movement of diverted material from the attack site to its intended customer.

Variables $X_{1}$ and $X_{2}$ are only used in the Outsider Attack and Transportation Diversion models. Variables $X_{4}, X_{5}, X_{7}$, and $X_{8}$ are used in the models for all scenario types.

Variable $X_{6}$ is used in the Outsider Attack, State Transfer, and Transportation Diversion scenarios. Variable $X_{3}$ remains in the source code, but is no longer used; it was judged to be too statistically dependent on variables $X_{1}$ and $X_{2}$.

Variables $Y_{1}$ and $Y_{2}$ mainly measure vulnerability to insider diversion. Variables $Y_{3}$ through $Y_{6}$ mainly measures vulnerability to attack by an outside group. Variables $Y_{7}$ and $Y_{8}$ mainly measure recoverability after a successful diversion, but before the material can be transported to the end user. Variable $Y_{9}$ mainly measures internal checks and balances that might thwart an attempt by part of the host government to divert materials to a non-permitted recipient. Variable $Y_{10}$ mainly measures willingness of the state or sub-state groups to attempt a state-transfer of material to non-permitted recipients.

Variables $Y_{11}, Y_{12}, Y_{13}, Y_{14}, Y_{15}$, and $Y_{16}$ are specific to the Transportation Diversion scenario.

\section{Description of the Likelihood and Risk Matrices and of the Threat, Vulnerability, Likelihood, Consequence, and Risk Categories}

The values of the Threat and Vulnerability variables are defined using ordinary multiplication of the values of the threat and vulnerability sub-variables. The uncertainties of the Threat and Vulnerability variables are calculated using ordinary random variable arithmetic from probability theory (Ahmed et al. 1982). The Threat and Vulnerability variables are then converted from their numerical values to categorical values by a "binning" or histogram process. 
The $4 \times 4$ Likelihood matrix provides a sort of arithmetic on the Threat and Vulnerability categories, giving the rules defining the Likelihood category value associated with each combination a Threat category and a Vulnerability category. The process described in Ahmed (1982) is adapted to propagate the Threat and Vulnerability uncertainties through to the Likelihood categories.

LIKELIHOOD $=$ F(THREAT, VULNERABILITY $)=$ Likelihood of successful diversion

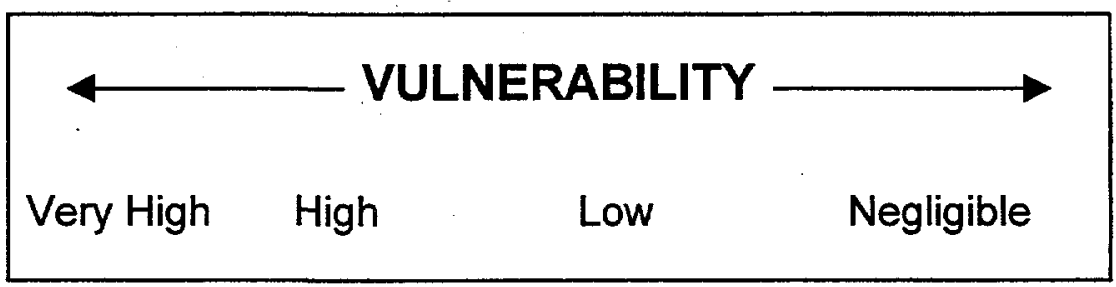

\begin{tabular}{|c|c|}
\hline$\varphi$ & Very High \\
\hline 字 & High \\
\hline 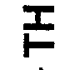 & Low \\
\hline$\nabla$ & Negligible \\
\hline
\end{tabular}

\begin{tabular}{|llll|}
\hline Very High & High & Medium & Low \\
Very High & High & Medium & Low \\
High & Medium & Low & Negligible \\
Low & Negligible & Negligible & Negligible \\
\hline
\end{tabular}

Notes of Threat Categories

\begin{tabular}{|l|l|}
\hline Very High & $\begin{array}{l}\text { Some organization or individual has intent and capabilities to plan and } \\
\text { execute an attack or diversion attempt }\end{array}$ \\
\hline High & $\begin{array}{l}\text { Some organization or individual has intent but with deficiencies in their } \\
\text { ability to plan and execute the attack or diversion }\end{array}$ \\
\hline Low & $\begin{array}{l}\text { Some organization or individual has intent but with no significant } \\
\text { capability to plan and execute the attack or diversion }\end{array}$ \\
\hline Negligible & $\begin{array}{l}\text { No organization or individual has the intent to plan and execute an attack } \\
\text { or diversion attempt }\end{array}$ \\
\hline
\end{tabular}


Notes on Vulnerability Categories

\begin{tabular}{|l|l|}
\hline Very High & $\begin{array}{l}\text { Site/Facility location, physical protections, and monitoring have significant } \\
\text { and known deficiencies that render it vulnerable to an attack or diversion } \\
\text { attempt }\end{array}$ \\
\hline High & $\begin{array}{l}\text { Site/Facility location, physical protections, and monitoring have significant } \\
\text { but not well-known deficiencies that render it vulnerable to an attack or } \\
\text { diversion attempt, or less significant known deficiencies }\end{array}$ \\
\hline Low & $\begin{array}{l}\text { Site/Facility location, physical protections, and monitoring have less } \\
\text { significant and not well-known deficiencies that render it vulnerable an } \\
\text { attack or diversion attempt }\end{array}$ \\
\hline Negligible & $\begin{array}{l}\text { Site/Facility location, physical protections, and monitoring have no } \\
\text { significant deficiencies }\end{array}$ \\
\hline
\end{tabular}

The $5 \times 5$ Risk matrix provides the "arithmetic" for converting each combination of Likelihood category and Consequence category to a corresponding Risk category. As before, the Ahmed (1982) process is adapted to propagate Likelihood and

Consequence category uncertainties through to the Risk category output of the model.

RISK = F(LIKELIHOOD, CONSEQUENCE) $=$ Overall risk associated with diversion attempts

\begin{tabular}{|c|c|}
\hline 4 & Very High \\
\hline 0 & High \\
\hline I & Medium \\
\hline $\bar{\Xi}$ & Low \\
\hline$\frac{1}{\nabla}+x-1$ & Negligible \\
\hline
\end{tabular}

\begin{tabular}{|ccccc|}
\hline & CONSEQUENCE & \\
Very High & High & Medium & Low & Negligible \\
\hline
\end{tabular}

\begin{tabular}{|lllll|}
\hline Very High & High & Medium & Low & Negligible \\
Very High & High & Medium & Low & Negligible \\
High & Medium & Low & Low & Negligible \\
High & Medium & Low & Low & Negligible \\
Medium & Low & Negligible & Negligible & Negligible \\
\hline
\end{tabular}


Notes on Consequence Categories

\begin{tabular}{|l|l|}
\hline Very High & Theft/Diversion of assembled weapons (IAEA Attractiveness Level A) \\
\hline High & $\begin{array}{l}\text { Theft/Diversion of large amounts of fissile material easily convertible } \\
\text { to weapons (IAEA Attractiveness Level B \& C, Categories I, II, or III) }\end{array}$ \\
\hline Medium & $\begin{array}{l}\text { Theft/Diversion of small amounts of easily convertible or large } \\
\text { amounts of difficult to convert material }\end{array}$ \\
\hline Low & Theft/Diversion of small amounts of difficult to convert material \\
\hline Negligible & Theft/Diversion of fission products or other non-weapons material \\
\hline
\end{tabular}

Notes of Likelihood Categories

\begin{tabular}{|l|l|}
\hline Likelihood Category & $\begin{array}{l}\text { Approximate Probability of Successful Attack during a } \\
\text { given Year }\end{array}$ \\
\hline Very High & 0.1 \\
\hline High & 0.01 \\
\hline Medium & 0.001 \\
\hline Low & 0.0001 \\
\hline Negligible & 0.00003 \\
\hline
\end{tabular}

\section{References}

Ahmed, Shahid, R. E. Clark, and D. R. Metcalf. 1982. "A Method for Propagating Uncertainty in Probabilistic Risk Assessment." Nuclear Technology, 59(2):238-245. 
Appendix B

\section{Model Implementation}




\section{APPENDIX B - Model Implementation}

\section{Details of the Model Implementation}

The current model attempts to describe the threats and vulnerabilities for each of the generic diversion scenarios in terms of a few variables that are approximately independent of each other. Each of the variables can take on a small number of values (two to four) which are defined qualitatively by the input screens presented to the evaluator. The variables are treated formally as random variables, so the evaluator is required to assign "probabilities" or "weights" to each of the possible values of the variables. The values of all of the threat sub-variables are multiplied together to define the overall threat variable; the values of the vulnerability sub-variables are multiplied together to define the vulnerability variable. The "multiplications" are done using convolution of the underlying sub-variables following the standard definition of multiplication of random variables in probability theory.

Let $X_{i}$ be one of the properties or sub-measures we are using to characterize a threat or, similarly, let $Y_{i}$ be a site/facility vulnerability sub-measure. We will assign a value from the interval $\left[a_{i}, b_{i}\right]$ to $X_{i}$ or $Y_{i}$, where $a_{i}<b_{i}$. In our model, $a_{i}$ is always non-negative, but may be equal to zero, and $b_{i}$ is always less than or equal to 3 . Then we define the overall aggregate measures of threat or vulnerability to be

$$
X=X_{1}{ }^{*} X_{2}{ }^{*} \ldots{ }^{*} X_{n},
$$

and

$$
Y=Y_{1} * Y_{2} * \ldots{ }^{*} Y_{m}
$$

The value of $X$ will lie between $A$ and $B$ where

$$
\begin{aligned}
& A=a_{1}{ }^{*} a_{2}{ }^{*} \ldots{ }^{*} a_{n} \text {, the product of the interval minimum values, and } \\
& B=b_{1}{ }^{*} b_{2}{ }^{*} \ldots{ }^{*} b_{n} \text {, product of the maximum values, and }
\end{aligned}
$$

similarly, the value of $Y$ will lie between $C$ and $D$ where

$$
\mathrm{C}=\mathrm{C}_{1}{ }^{*} \mathrm{C}_{2} * \ldots{ }^{*} \mathrm{C}_{\mathrm{m}}
$$

and

$$
D=d_{1}{ }^{*} d_{2} * \ldots{ }^{*} d_{m} \text {, and }
$$

the $c_{j}$ 's and the $d_{i}$ 's are the corresponding interval minimum and maximum values for the vulnerability sub-measures. 
Larger values of the $X_{i}$ 's and $X$ will correspond to larger threats, and larger values of the $Y_{i}{ }^{\prime}$ s and $Y$ will correspond to higher vulnerabilities. See Appendix $A$ for detailed descriptions of the threat and vulnerability sub-measures and their use within the models for the various scenario types.

The range of possible values of $X$, i.e., the interval [ $A, B$ ], and the range of possible values of $Y$, the interval $[C, D$.$] , will each be partitioned into four categorical sub-$ intervals, labeled Negligible, Low, High, and Very High. A four by four matrix will associate with each threat category and vulnerability category pair, one of five likelihood categories, labeled Negligible, Low, Medium, High, and Very High. The evaluator will directly provide an estimate of the likely consequences as one of five categories, also labeled Negligible, Low, Medium, High, and Very High. Finally, a five by five matrix will take each likelihood and consequences category pair and return one of five risk categories, also labeled Negligible, Low, Medium, High, and Very High. See Appendix A for a description of the likelihood and risk matrices and approximate definitions of the threat, vulnerability, likelihood, and consequence categories.

If a threat characteristic $X_{i}$ is absolutely required for the development of intent, for planning, obtaining resources, and execution of the attack/diversion, then the lack of that characteristic can be assigned the value $X_{i}=a_{i}=0$, which will have the effect of zeroing out the aggregate threat measure.

If a threat characteristic $X_{i}$ is relatively more important in determining the likelihood of successful diversion, then the maximum value $b_{i}$ assignable to $X_{i}$ should be larger than the $b_{j}$ 's for other characteristics.

If the lack of a particular vulnerability characteristic $Y_{i}$ is absolutely protective against a successful diversion, then that vulnerability can be assigned the value $Y_{i}=c_{i}=0$, which again will have the effect of zeroing out the aggregate vulnerability of the site/facility.

If a vulnerability characteristic $Y_{i}$ is relatively more important, then the maximum value $d_{i}$ assignable to $Y_{i}$ should be larger than the $d_{j}$ 's for other vulnerability characteristics.

\section{Propagation of Input Uncertainties}

One of the basic features of this model is the consistent propagation of input uncertainties through the calculation to the final estimate of the risk of successful diversion. Each of the threat and vulnerability sub-measures, and the likelihood, consequences, and risk measures are treated as random variables. Expert user input defines the threat and vulnerability sub-measures and the consequence measure random variables. The probability distributions of the likelihood and risk measures are computed from representation of uncertainty in the expert user input for the other variables. 
Appendix $\mathrm{D}$ has a brief discussion of the concepts from probability theory used in the implementation of this model.

The expert user defines the random variable $\mathrm{X}_{4}$, for instance, by providing probabilities or "weights" for both of the allowed values of that variable. Since they are to be probabilities, they must lie between 0 and 1 and must add up to 1 . The input solicitation subroutines enforce the second requirement (but, currently, not the first). Once the threat and vulnerability sub-measure random variables have been defined, the threat and vulnerability measures (also random variables) are calculated using the usual rules for random variable arithmetic. Those values are converted to categorical random variables (with values "negligible", "low", etc.) and the likelihood matrix is used to calculate the likelihood categorical random variable. Expert user input is solicited to define the Consequences categorical random variable and the values of the Likelihood and Consequences categorical random variables are plugged into the risk matrix to compute the Risk categorical random variable.

Our use of the phrase "categorical random variable" needs a bit of explanation. The usual definition of a random variable $X$ is that $X$ is a function from some probability space (i.e., a sample space on which a probability function satisfying the usual axioms is defined). An equivalent definition is that a random variable $X$ is an assignment of probabilities to some set of real numbers that satisfies the usual axioms for probability functions. By "categorical random variable", we mean a set of categories (in our case, four or five categories) to which we have assigned non-negative numbers that add up to one.

We use two "categorical" matrices to define what amounts to an arithmetic or an algebra on the threat, vulnerability, likelihood, consequence, and risk categories. For instance the $4 \times 4$ Likelihood matrix tells us which of the 5 likelihood categories corresponds to each of the 16 combinations of one of the 4 threat categories and one of the 4 vulnerability categories. The probability assigned one of the 16 entries in the Likelihood matrix will be the product of the probabilities of the corresponding threat and vulnerability categories. Finally, we calculate the probability of each of the Likelihood categories by adding the calculated probabilities for the Likelihood matrix entries for that category.

The uncertainty-handling scheme can even handle the case where the expert has no knowledge with which to choose between various values of the threat or vulnerability sub-measure or the consequences categorical measure. In that case, the expert simply assigns equal probabilities to all allowed values of the random variable.

\section{Interpretation of Results}

The basic output of the model is an assignment of probabilities to each of the five Risk categories. If most of the probability (i.e., $75 \%$ or more) is assigned to one or two categories that should suggest that the impact of evaluator uncertainties is acceptably 
low for us to put some credence in the risk estimation. If significant probability is assigned to three or more of the five categories, that should suggest that residual uncertainty is great. One response might be collection of information to reduce that residual uncertainty.

\section{Implementation Notes and Lessons Learned from Development of Prototype Risk Evaluation Program}

This Section contains a brief conceptual description of the diversion risk model, a brief description of the structure of the prototype evaluation programs and crucial implementation details. Additionally developmental problems and known model problems are discussed. Some suggestions regarding the possible next versions of the evaluation program are provided.

\section{Conceptual Description of the Diversion Risk Model}

The prototype evaluation programs implement a diversion risk model. Briefly, for each of the five scenario types (insider theft, outsider theft, outsider attack, state transfer, transportation attack), there is a set of threat sub-measures, $\left\{X_{1}, X_{2}, \ldots, X_{k}\right\}$, and a set of vulnerability sub-measures, $\left\{Y_{1}, Y_{2}, \ldots, Y_{n}\right\}$. Both the threat measure $X$ and the vulnerability measure $Y$ are multiplicative, that is,

$$
\begin{aligned}
& X=X_{1}{ }^{*} X_{2}{ }^{*} \ldots{ }^{*} X_{k} \text { and } \\
& Y=Y_{1}{ }^{*} Y_{2}{ }^{*} \ldots \ldots{ }^{*} Y_{n} .
\end{aligned}
$$

Each threat sub-measure $X_{i}$ and each vulnerability sub-measure $Y_{j}$ is assigned one of a discrete set of values in the range $0<=X_{i}<=X_{i}$ max (and similarly for $Y_{j}$ ). In addition, if the property measured by $X_{i}$ is absolutely essential to the existence of a legitimate threat, then the assignment of $X_{i}=0$ will indicate that that property is not present and, because of the multiplicative form of $X, X$ will also be equal to zero and the overall threat will be non-existent (or negligible). Similarly, if a particular vulnerability property is absolutely protective against diversion, then the assignment $Y_{j}=0$ has the effect of zeroing out $Y$ and setting the vulnerability $Y=0$ (or negligible).

The ranges of possible numerical values for $X$ and $Y$ are each split into four subintervals, corresponding to qualitative categories: Negligible, Low, High, and Very High. A $4 \times 4$ matrix is used to convert the threat and vulnerability categories into five likelihood categories: Negligible, Low, Medium, High, and Very High. The user of the evaluation program assigns one of five categories (also Negligible, Low, Medium, High, and Very High) to diversion consequences based on the type and quantity of material diverted. Finally, a $5 \times 5$ matrix is used to convert the likelihood and consequence categories to a site/facility diversion risk category (also Negligible, Low, Medium, High, and Very High). 


\section{Prototype Evaluation Program Implementation Notes}

The prototype evaluation programs were written in Microsoft QBasic and compiled using Borland TurboBasic. They have a relatively clunky text-based user interface. They implement the proposed threat/vulnerability measure aggregation scheme, including handling of evaluator uncertainty. Evaluator uncertainty is handled by requiring the evaluator to assign a probability, representing his/her degree of belief, to each of the possible discrete values that a threat or vulnerability or consequence measure can take on. Because of memory limitations of QBasic, five separate programs were developed, one for each of the scenario types. Also because of the memory limitations, some variables which were originally double precision were changed to single precision, with the eventual result that although much of the output is shown in double precision, only the first 6 or 7 digits are meaningful.

Initially, programs were developed for the Insider Theft, Outsider Theft, Outsider Attack, and State. Transfer scenario types. Somewhat later, the development of the Transportation Attack model required the creation of additional vulnerability submeasures $Y_{11}$ through $Y_{16}$. Input screens for these measures appear in the source code XP_EVAL4.ASC for this model, but not in the source code of the four earlier programs.

Each of the five programs consists of a short main program, four major subroutines, and 18 threat/vulnerability input soliciting subroutines ( 24 input subroutines for the Transport Attack model). The main program declares and dimensions variables, initializes some of the variables, requests the output filename and the site/facility name, and then branches to subroutine 1000 .

Subroutine 1000 calls the appropriate input solicitation subroutines. All input provided by the user is echoed to the output file. When all of the threat and vulnerability submeasure weights have been inputted by the user, subroutine 1000 calculates the submeasure weight (or probability) corresponding to each subset of sub-measure values. Of course, each set of sub-measure values will determine a particular value of the measure (with its associated sub-measure weight).

For example, for the insider theft program, the threat sub-measures are $X_{4}, X_{5}, X_{7}$, and $X_{8}$. The corresponding sub-measure value variables are

$$
\begin{aligned}
& X_{4} \vee(1)=0.0 \text { and } X_{4} V(2)=1.0 \\
& X_{5} V(1)=0.0, X_{5} \vee(2)=0.5, X_{5} \vee(3)=1.0 \text {, and } X_{5} V(4)=2.0 \\
& X_{7} V(1)=0.5 \text { and } X_{7} \vee(2)=1.0 \\
& X_{8} \vee(1)=0.5, X_{8} \vee(2)=1.0, \text { and } X_{8} \vee(3)=2.0
\end{aligned}
$$


The subroutines $140,150,170$, and 180 asks the evaluator to provide the corresponding weights (i.e., probabilities) by assigning values to the variables

$$
\begin{aligned}
& X_{4}(1) \text { and } X_{4}(2) ; \\
& X_{5}(1), X_{5}(2), X_{5}(3), \text { and } X_{5}(4) ; \\
& X_{7}(1) \text { and } X_{7}(2) ; \text { and } \\
& X_{8}(1), X_{8}(2), \text { and } X_{8}(3) .
\end{aligned}
$$

Inside the nested loop

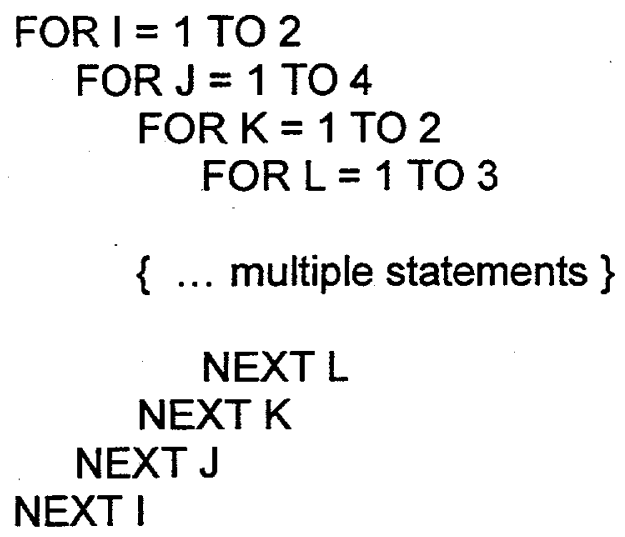

the variables ITTHREATV\#(I,J,K,L) and ITTHREATP\#(I,J,K,L) are calculated.

ITTHREATV\#(I,J,K,L) is the value of the threat variable $X=X_{4}{ }^{*} X_{5}{ }^{*} X_{7}{ }^{*} X_{8}$

for the $I, J, K, L$ set of threat sub-measure values. It is computed as the product of the threat sub-measure values for the I,J,K,L set of threat sub-measure values.

ITTHREATP\# $(I, J, K, L)$ is the corresponding probability assigned to that threat variable value. It is computed as the product of the user-supplied weights for the $\mathrm{I}, \mathrm{J}, \mathrm{K}, \mathrm{L}$ set of threat sub-measure values.

Then the probability ITTHREATP\# associated with the threat variable value ITTHREATV\# is added to all of the probabilities previously assigned to the same threat variable value. This cumulative probability value is assigned to the variable ITTHREATPROB\#, which is a dimension 101 vector variable. This calculation essentially constructs a histogram from the probabilities for the same or approximately the same threat variable values. This particular routine for collecting probabilities for approximately-equal threat variable values uses a quirk of $Q B a s i c$ and TurboBasic. If $X$ is a 101 dimension vector, QBasic will take the statement $X(2.3)=0.02$ and assign the value 0.02 to $X(2) ; X(1.6)=0.04$ will assign the value 0.04 to $X(2)$. Thus, if we have computed a probability ITTHREATP\# $(2,2,1,2)=0.0432$ for the threat variable value 0.25 , the statement 
ITTHREATPROB\#(ITTHREATV\# $\left.(2,2,1,2)^{*} 25+1\right)=$

ITTHREATPROB\#(ITTHREATV\# $\left.(2,2,1,2){ }^{*} 25+1\right)+$ ITTHREATP\# $(2,2,1,2)$

will add the probability 0.0432 to previously accumulated value of

ITTHREATPROB\#(7), since ITTHREATV\# $(2,2,1,2)=0.25$ and $25^{*} 0.25+1=7.25$, which rounds to 7 .

A couple of statements printing these intermediate calculations to the output file are commented out, since they aren't needed in the debugged program and consume a lot of paper.

Similar calculations of the vulnerability variables are also performed inside their own nested loops.

Finally, after all of the calculations in the nested loops have been performed, the cumulated (histogram) probabilities are printed out to the output files. These statements are now commented out, again to save paper.

With the cumulated probability values of the threat and vulnerability variables now calculated, subroutine 1000 calls subroutine 5000 .

Subroutine 5000 collects the cumulated probability values for the threat variable into four classes, which are then used to assign probabilities to the four threat categories (negligible, low, high, and very high). Similarly, the cumulated probability values for the vulnerability variable are collected into for classes and assigned to the four vulnerability categories (also negligible, low, high, and very high). Control is returned to subroutine 1000 that immediately calls subroutine 5100 .

The following table summarizes the numerical division points between the threat and vulnerability categories used in the prototype evaluation programs.

The establishment of these numerical division points is part of the process of tuning/calibrating the model so that it emulates the expert estimates of risk.

Subroutine 5100 computes the Likelihood matrix probability values and accumulates them into the five Likelihood category probabilities and prints the category probabilities out to the output file. Subroutine 5100 then returns control to subroutine 1000 .

Subroutine 1000 calls subroutine 5200 , which solicits input from the evaluator for the Consequence measure, given as weights/probabilities assigned to each of the five Consequence categories. This input is echoed to output. Finally, Risk Matrix probabilities are calculated and then accumulated into the five Risk category probabilities, which are printed out to the output file. Control is returned to subroutine 1000 and then to the main program, which then immediately terminates normally. 


\begin{tabular}{|c|c|c|c|c|c|c|c|c|c|c|}
\hline \multirow[t]{2}{*}{ CATEGORY } & \multicolumn{2}{|c|}{ IT_EVAL } & \multicolumn{2}{|c|}{ OT_EVAL } & \multicolumn{2}{|c|}{ OA_EVAL } & \multicolumn{2}{|c|}{ ST_EVAL } & \multicolumn{2}{|c|}{ XP_EVAL } \\
\hline & Threat & Vulnerability & Threat & Vulnerability & Threat & Vulnerability & Threat & Vulnerability & Threat & Vulnerability \\
\hline & 0.00 & 0.00 & 0.00 & 0.00 & 0.00 & 0.00 & 0.00 & 0.00 & 0.00 & 0.00 \\
\hline \multicolumn{11}{|l|}{ Negligible } \\
\hline & 0.62 & 0.62 & 0.62 & 2.50 & 0.83 & 1.25 & 0.875 & 0.31 & 0.50 & 0.83 \\
\hline \multicolumn{11}{|l|}{ Low } \\
\hline & 1.26 & 1.26 & 1.26 & 4.50 & 1.83 & 2.25 & 2.375 & 0.83 & 1.83 & 1.50 \\
\hline \multicolumn{11}{|l|}{ High } \\
\hline & 2.46 & 2.46 & 2.46 & 8.50 & 3.17 & 4.25 & 4.292 & 1.23 & 3.17 & 2.83 \\
\hline \multicolumn{11}{|l|}{ Very High } \\
\hline & 4.00 & 4.00 & 4.00 & 96.00 & 32.00 & 48.00 & 8.00 & 2.00 & 33.30 & 33.30 \\
\hline
\end{tabular}

\section{B. 8}


Appendix C

\section{Basic Concepts of Probability Theory}




\section{APPENDIX C -Basic Concepts of Probability Theory}

Consider some set of possible outcomes of an experiment. For instance, the experiment might be to flip a coin three times. There are eight possible outcomes of this experiment: the set $S=\{\mathrm{HHH}, \mathrm{HHT}, \mathrm{HTH}, \mathrm{HTT}, \mathrm{THH}, \mathrm{THT}, \mathrm{TTH}, \mathrm{TT}\}$. If the coin is honest, that is, if the probability of a head on a single flip is 0.5 , then each of the eight outcomes of our experiment can be assigned a probability of $1 / 8=0.125$. The probability of getting 2 heads and 1 tail in the three flips is calculated by adding the probabilities of the three different ways of getting 2 Heads and 1 Tail:

$$
P(2 \text { Heads })=P(H H T)+P(H T H)+P(T H H)=3 / 8=0.375
$$

In general, we will call any subset $E$ of $S$ an event and will compute its probability by adding up all of the probabilities of the primitive outcomes that comprise the event.

Even more generally, any time we have a sample space $S$ and a collection of subsets $E$, the events, of the sample space, we can define a probability space on $S$ and the collection of events by defining a function $\mathrm{P}$ that assigns probabilities to each event, which satisfy the three Kolmogorov axioms:

$$
\text { Axiom } 1-0<=P(E)<=1
$$

Axiom $2-P(S)=1$

Axiom 3 -- If $E_{1}, E_{2}, \ldots$ is a possibly infinite sequence of mutually exclusive events, then the probability of the union of the $E_{i}$ 's is equal to the sum of the probabilities of the $E_{i}$ 's.

A mathematical nicety left out of the description above is that the collection of events has to form a sigma-algebra of subsets of $\mathrm{S}$. Roughly speaking this means that finite intersections of events and possibly infinite unions of events must also be events (i.e., must also belong to the collection). This condition is necessary for Axiom 3 to make sense.

In our application, the sample spaces are very simple. They consist of the two, three, or four discrete numerical values of each of the Threat and Vulnerability sub-measures or of the four or five "categorical" values of the overall Threat, Vulnerability, Likelihood, Consequence, and Risk measures.

A random variable $X$ is a function from the set of events to the real numbers. It can also be considered to be an assignment of probabilities to some subset of the real numbers, which satisfies the Kolmogorov axioms. We broaden the usual definition of a random 
variable to include "categorical" random variables, which mean an assignment of probabilities satisfying the axioms to some set of categories.

If two random variables $X_{1}$ and $X_{2}$ are independent, then the joint probability that $X_{1}=2$ and $X_{2}=3$ is given by

$$
P\left(X_{1}=2 \text { and } X_{2}=3\right)=P\left(X_{1}=2\right)^{*} P\left(X_{2}=3\right),
$$

the multiplication rule for independent events. On the other hand, if they are not independent, then

$$
P\left(X_{1}=2 \text { and } X_{2}=3\right)=P\left(X_{1}=2\right)^{\star} P\left(X_{2}=3 \mid X_{1}=2\right) \text {, }
$$

where the second probability on the right hand side is the conditional probability that $X_{2}=3$, given that $X^{1}$ is known to be equal to 2 .

The conditional probability $P\left(X_{2}=3 \mid X_{1}=2\right)$ is formally defined to be

$$
P\left(X_{2}=3 \mid X_{1}=2\right)=P\left(X_{1}=2 \text { and } X_{2}=3\right) / P\left(X_{1}=2\right) \text {, }
$$

which clearly requires that $P\left(X_{1}=2\right)$ not be equal to zero.

If we abandoned the model implementation assumption that the various threat and vulnerability sub-variables are independent, then the evaluators would have to estimate not only the values of the variables (as probabilities), but also conditional probabilities for combinations of values of the variables. This would greatly increase the amount of input required and it would probably be more difficult for the evaluators to provide reasonable estimates of the conditional probabilities.

If $X_{1}$ and $X_{2}$ are random variables as before, then the probability that the product $X_{1}{ }^{*} X_{2}=d$ is given by the convolution sum

$$
P\left(X_{1}{ }^{*} X_{2}=d\right)=\text { Sum of terms } P\left(X_{1}=c\right)^{\star} P\left(X_{2}=d / c\right),
$$

where the sum is taken over all values of $\mathrm{c}$ for which both probabilities on the right hand side make sense.

Our programs uses a slightly different and somewhat simpler way of implementing an approximate convolution of the two random variables. We calculate all probabilities of the form

$$
P\left(X_{1}{ }^{*} X_{2}=a^{*} b\right)=P\left(X_{1}=a\right)^{*} P\left(X_{2}=b\right)
$$


and then bin together or form a histogram that collects all of the values of probabilities for $a^{*} b$ approximately equal to $c$. That is,

$P\left(X_{1}{ }^{*} X_{2}=c\right)=$ Sum of terms of the form $P\left(X_{1}=a\right)^{*} P\left(X_{2}=b\right)$, with the sum being taken over all pairs of values $a$ and $b$ with $a * b$ approximately equal to $c$. 
Appendix D

Source Code for Scenarios 


\section{APPENDIX D - Source Code for Scenarios}

\section{Source Code for the Five Diversion Scenarios}

Appendix B contains a description of how the source code for the five diversion scenarios is organized.

\section{Source code for the Insider Theft Scenario}

This is a prototype, with a text-based user interface. It implements

- the proposed threat/vulnerability measure aggregation scheme,

- including handling of evaluator uncertainty

- The program will begin displaying the relevant threat and

- vulnerability sub-measures one by one

- and prompting the evaluator for his/her estimates of the weights (or probabilities) to be assigned to each of the possible sub-measure values. For each sub-measure, these weights should sum to 1 . The program will solicit weights for the consequence measure, which is based on the quantity and attractiveness of the nuclear materials available for diversion at the site.

When the input for all relevant sub-measures has been entered, the program will compute the aggregate values, will assign categorical values to the threat and vulnerability measures, and will compute the corresponding diversion risk measure. The evaluator-assigned uncertainties will be propagated all the way through to the diversion risk measure.

\section{DEFSNG A-H, O-Z}

DEFINT I-N

OPTION BASE 1

EPS $=.001$

DIM X1(3) ' Long Standing Conflict - WEIGHTS

DIM X2(2) 'Political/ideological motivations - WEIGHTS

DIM X3(3) 'Religious motivations -- WEIGHTS

DIM X4(2) 'Perception of target vulnerability -- WEIGHTS

DIM X5(4) 'Availability of resources - WEIGHTS

DIM X6(3) 'Training and leadership - WEIGHTS

DIM X7(2) 'Knowledge and intelligence -- WEIGHTS

DIM X8(3) 'Site/facility socio-economic conditions - WEIGHTS

DIM Y1(3) ' Material surveillance - WEIGHTS 


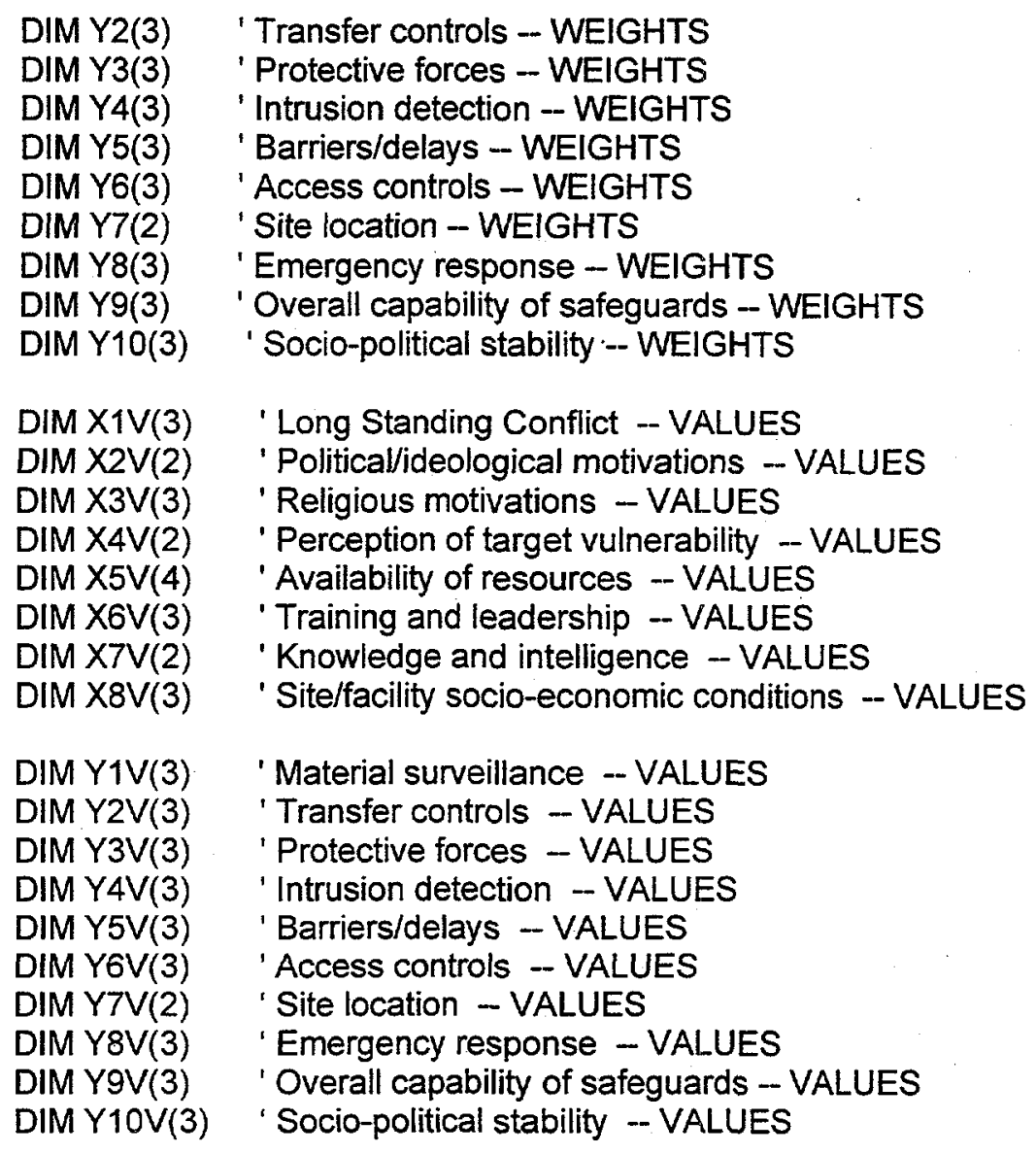

FOR I = 1 TO 3: READ X1V(I): NEXT I

DATA $0.5,1.0,2.0$

FOR I = 1 TO 2: READ X2V(I): NEXT I

DATA $0.5,1.0$

FOR I = 1 TO 3: READ X3V(I): NEXT I

DATA $0.5,1.0,2.0$

FOR I = 1 TO 2: READ X4V(I): NEXT I

DATA $0.5,1.5$

FOR I = 1 TO 4: READ X5V(I): NEXT I

DATA $0.0,0.5,1.0,2.0$

FOR I = 1 TO 3: READ X6V(I): NEXT I

DATA $0.5,1.0,2.0$

FOR I = 1 TO 2: READ XTV(I): NEXT I

DATA $0.5,1.5$

FOR I = 1 TO 3: READ X8V(I): NEXT I

DATA $0.75,1.0,1.5$

FOR I = 1 TO 3: READ Y1V(I): NEXT I

DATA $0.5,1.0,2.0$

FOR I = 1 TO 3: READ Y2V(I): NEXT I

DATA $0.5,1.0,2.0$

FOR I = 1 TO 3: READ Y3V(I): NEXT I

DATA $0.5,1.0,2.0$ 
FOR I = 1 TO 3: READ Y4V(I): NEXT I

DATA $0.5,1.0,2.0$

FOR I = 1 TO 3: READ Y5V(I): NEXT I

DATA 0.5, 1.0, 2.0

FOR I = 1 TO 3: READ Y6V(I): NEXT I

DATA 0.5, 1.0, 2.0

FOR I = 1 TO 2: READ YTV(I): NEXT I

DATA $0.5,1.5$

FOR I = 1 TO 3: READ Y8V(I): NEXT I

DATA $0.5,1.0,2.0$

FOR I = 1 TO 3: READ Y9V(1): NEXT I

DATA $0.5,1.0,2.0$

FOR I = 1 TO 3: READ Y10V(I): NEXT I

DATA $0.5,1.0,2.0$

DIM ITTHREATPROB!(101)

DIM ITVULNPROB!(101)

DIM THREAT(4) 'Threat category probabilities

DIM VULN(4) 'Vulnerability category probabilities

DIM LHOOD!(5) 'Likelihood category probabilities

DIM LMAT! $(4,4)$ 'Likelihood matrix probabilities

DIM CONSEQ(5) 'Consequence category probabilities

DIM RISK $(5,5)$ 'Risk matrix probabilities

DIM RISKCAT(5) 'Risk category probabilities

DIM scentype\$(4) ' Scenario type names

FOR I = 1 TO 4: READ scentype\$(I): NEXT I

DATA "Insider theft", "Outsider theft", "Outsider attack", "State transfer"

INPUT "Enter name of output file "; outfile\$

OPEN outfile\$ FOR OUTPUT AS \#1

' Define printout formats

a2\$ = "\&\#\#\# \&\#.\#"

a3\$ = "\&\#.\#\# \&\#.\#\# \& \#.\#\#"

a4\$ = "\&\#.\#\# \& \#.\#\# \&\#.\#\# \& \#.\#"

tV $\$=$ "\& \#\#.\# \&\#.\#"

$C \$=" \& \#$

IC $\$=" \& \# \& \#$

INPUT "Enter site/facility name "; sitename\$

PRINT \#1, "Evaluation of site/facility: "; sitename\$

PRINT \#1, "For scenario type: $\quad$ "; scentype\$(1)

PRINT \#1, "Output file name: "; outfile\$

PRINT \#1, "Program and version: Version 4 of IT_EVAL"

PRINT \#1,

gosub 1000 
STOP

' Subroutines 110 through 290 solicit evaluator input for Threat and

' Vulnerability sub-measures. Subroutine 100 explains that the Threat

' and Vulnerability sub-measures are all treated as random variables,

' requiring that probabilities (or weights) summing to 1 must be assigned

' to each of the numerical values taken on by that sub-measure.

\section{CLS}

PRINT "The Threat and Vulnerability sub-measures used in this scheme" PRINT "for estimating the risk of diversion of nuclear materials are" PRINT "all treated as random variables, requiring that probabilities" PRINT "(or weights) summing to 1 must be assigned to each of the" PRINT "numerical values taken on by that sub-measure. The input routines" PRINT "will enforce this requirement."

PRINT '"'

PRINT "In addition, the assignment of probabilities to the sub-measure" PRINT "can be used to represent the evaluators uncertainty about the" PRINT "correct value of the sub-measure. For instance, in the absence of" PRINT "any information about the true value of the sub-measure" PRINT "(i.e., complete uncertainty), the evaluator should assign equal" PRINT "weights to all of the sub-measure values."

PRINT "'!

PRINT "Touch any key to continue"

DO

LOOP UNTIL INKEY\$ $<>$ '"'

RETURN

110 CLS

PRINT "Existence of a Long Standing Conflict "

PRINT "'"

PRINT " $\quad X_{-} 1=0.5$, no underlying conflict"

PRINT " $\quad X_{-1}^{-1}=1.0$, recently developed conflict (decades-long duration)"

PRINT " X_1 $=2.0$, long standing conflict (centuries-long duration)"

PRINT "'

PRINT "*Long Standing Conflict* is shorthand for aspects of intensity " PRINT "and long duration in the motivation of the threat groups. It can " PRINT "derive from extreme religious prejudice, language/cultural " PRINT "differences, and/or from a long history of oppression, " PRINT "suppression, or warfare between two groups of people. Examples" PRINT "include Catholic Irish vs. Irish Protestants and the British"

PRINT "or the Basques vs. the Spanish or Islamic fundamentals vs. " PRINT "secular Moslem governments. "

PRINT "'"

INPUT "Input three X_1 weights "; X1(1), X1(2), X1(3)

IF ABS $(X 1(1)+X 1(2)+X 1(3)-1 !)>$ EPS THEN

PRINT "Your weights for $X \_1$ should add up to 1.0."

PRINT "Please repeat the weight assignment."

PRINT "Touch any key to continue"

DO 


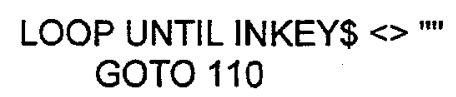

END IF

PRINT \#1, "Variable $X 1$ is Existence of a Long Standing Conflict"

PRINT \#1, USING a3\$; "X_1 values are "; X1V(1); " "; X1V(2); " "; X1V(3)

PRINT \#1, USING a3\$; "X_1 weights are "; X1(1); " "; X1(2); " "; X1(3)

PRINT \#1,

\section{RETURN}

120 CLS

PRINT "Political//deological Motivations"

PRINT "'"'

PRINT " X_2 $=0.5$, no political/ideological basis "

PRINT " X_2 $=1.0$, political/ideological motivations"

PRINT "'!

PRINT "Examples would be support for terrorism as a component "

PRINT "of the cold war struggle between Communism and the West."

PRINT "'!

INPUT "Input two $X \_2$ weights "; $X 2(1), X 2(2)$

IF ABS $(X 2(1)+X 2(\overline{2})-1$ !) $>$ EPS THEN

PRINT "Your weights for $X \_2$ should add up to 1.0."

PRINT "Please repeat the weight assignment."

PRINT "Touch any key to continue"

DO

LOOP UNTIL INKEYS <> '"'

GOTO 120

END IF

PRINT \#1, "Variable $X 2$ is Political/ldeological Motivations"

PRINT \#1, USING a2\$; "X_2 values are "; X2V(1); " "; X2V(2)

PRINT \#1, USING a2\$; "X_2 weights are "; X2(1); " "; X2(2)

PRINT \#1,

\section{RETURN}

- The threat sub-variable $X \_3$ is no longer being used, since we judged

' it to be statistically dependent with X_1 and X_2

130 CLS

PRINT "Religious Motivations"

PRINT " "

PRINT " $X \_3=0.5$, no religious animosity"

PRINT " $X 3=1.0$, some religious animosity"

PRINT " $\quad X \_3=2.0$, strong religious animosity"

PRINT "'"

PRINT "The intent here is to capture aspects of motivation distinct "

PRINT "from possible religious components of the "; blood; feud; " measure "

PRINT "above."

PRINT "'!

INPUT "Input three $X \_3$ weights "; $X 3(1), X 3(2), X 3(3)$

IF ABS(X3(1) + X3(2) + X3(3) - 1!) > EPS THEN

PRINT "Your weights for $X \_3$ should add up to 1.0." 
PRINT "Please repeat the weight assignment."

PRINT "Touch any key to continue"

DO

LOOP UNTIL INKEY\$ <> "'"

GOTO 130

END IF

PRINT \#1, "Variable X3 is Religious Motivations"

PRINT \#1, USING a3\$; "X_3 values are "; X3V(1); " "; X3V(2); " "; X3V(3)

PRINT \#1, USING a3\$; "X_3 weights are "; X3(1); " "; X3(2); " "; X3(3)

PRINT \#1,

RETURN

140 CLS

PRINT "Perception of target vulnerability "

PRINT "'"

PRINT " X_4 $=0.5$, facility not perceived as vulnerable "

PRINT " $X \_4=1.5$, facility perceived as vulnerable"

PRINT "'"

PRINT "The reasoning here is that, even for a threat group predisposed "

PRINT "to action, if a particular facility is perceived as not vulnerable,"

PRINT "they will direct their attention elsewhere."

PRINT "'"

INPUT "Input two X_4 weights "; X4(1), X4(2)

IF ABS $(X 4(1)+X 4(\overline{2})-1 !)>$ EPS THEN

PRINT "Your weights for $X \_4$ should add up to 1.0."

PRINT "Please repeat the weight assignment."

PRINT "Touch any key to continue"

DO

LOOP UNTIL INKEY\$ <> "'"

GOTO 140

END IF

PRINT \#1, "Variable X4 is Perception of Target Vulnerability"

PRINT \#1, USING a2\$; "X_4 values are "; X4V(1); " "; X4V(2)

PRINT \#1, USING a2\$; "X_4 weights are "; $X 4(1)$; " "; $X 4(2)$

PRINT \#1,

\section{RETURN}

- For the Insider Theft case we have modified Subroutine 150 so that

- X 5 takes on only the values 0.5 and 1.5 . For simplicity, we have simply

- set the probabilities of the 0.0 and 2.0 values to zero and changed the

- 1.0 value to 1.5 . The user is now solicited to provide probabilities

summing to 1.0 for the 0.5 and 1.5 values of $X 5 \mathrm{~V}$. The rationale is that

' the insider is never completely without resources and is also not likely

' to have the resources of a nation state.

150 CLS

PRINT "Availability of resources "

PRINT "'!

PRINT " $X 5=0.5$, modest, locally collected resources"

PRINT " $\quad X \_5=1.5$, intermediate, with outside support" 


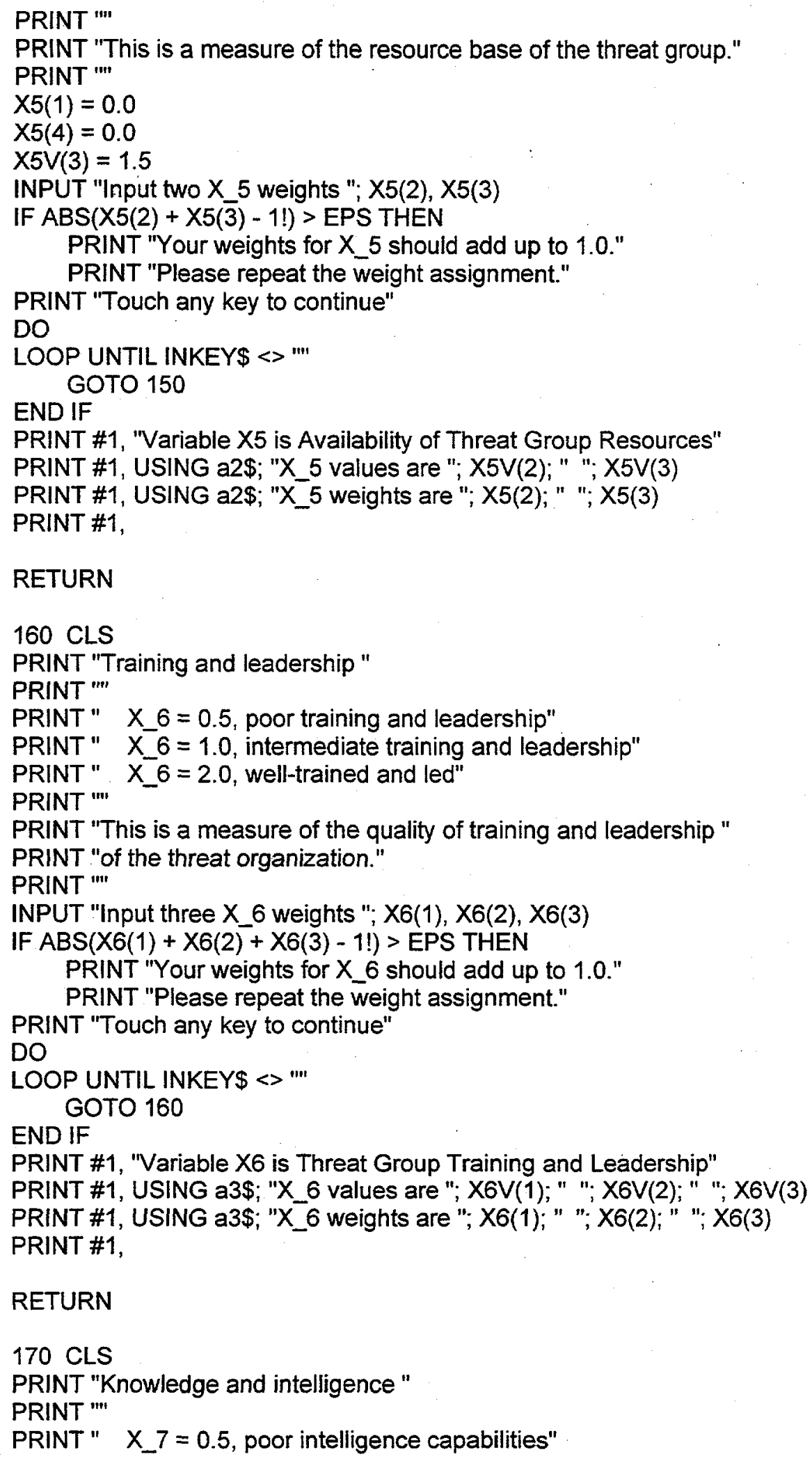




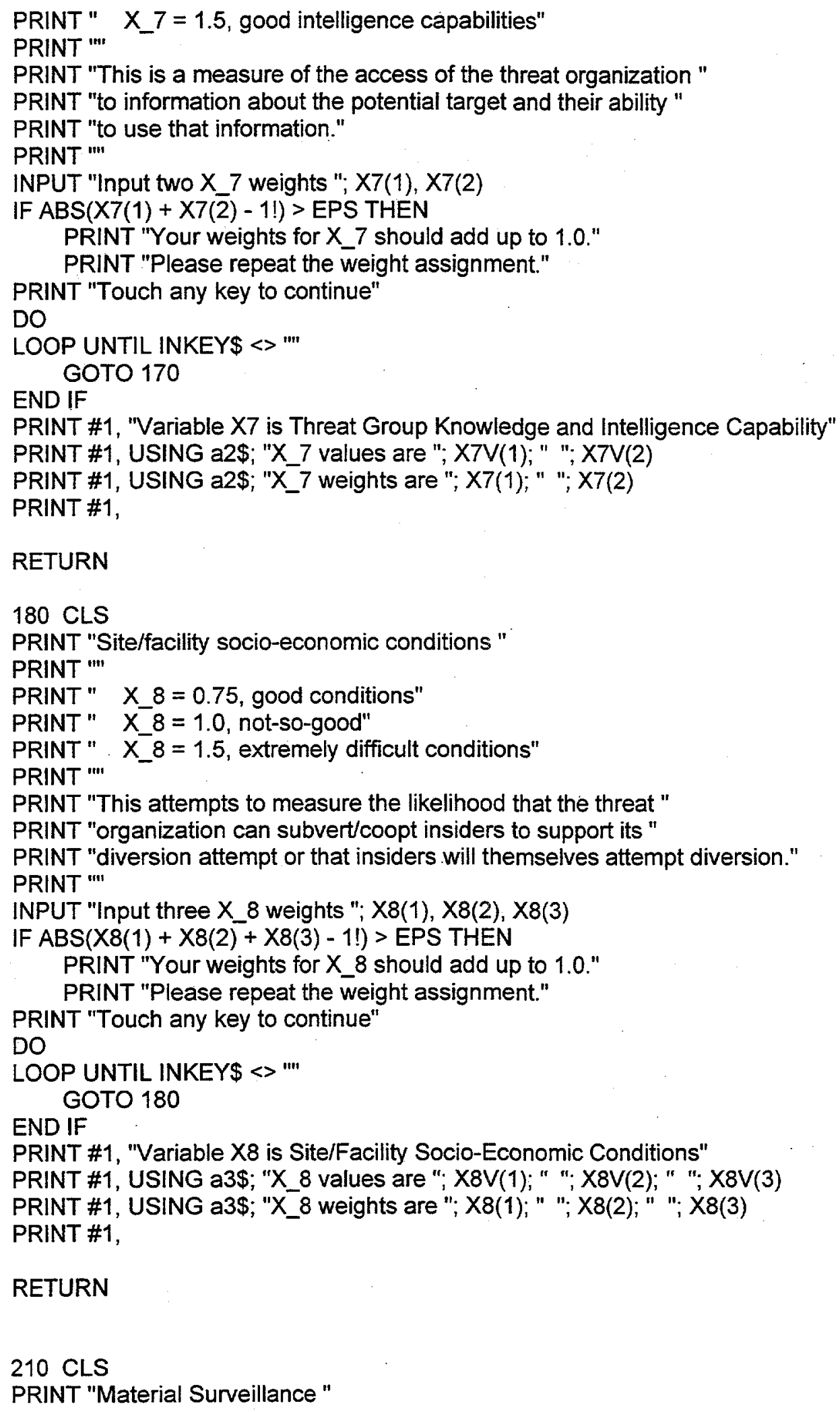

\section{RETURN}

180 CLS

PRINT "Site/facility socio-economic conditions "

PRINT '"'

PRINT " X_8 $=0.75$, good conditions"

PRINT" X_8 $=1.0$, not-so-good"

PRINT" $X \_8=1.5$, extremely difficult conditions"

PRINT "'"

PRINT "This attempts to measure the likelihood that the threat "

PRINT "organization can subvert/coopt insiders to support its "

PRINT "diversion attempt or that insiders will themselves attempt diversion."

PRINT "'!

INPUT "Input three $X \_8$ weights "; $X 8(1), X 8(2), X 8(3)$

IF ABS(X8(1) +X8(2) + X8(3) - 1!) > EPS THEN

PRINT "Your weights for $X \_8$ should add up to 1.0."

PRINT "Please repeat the weight assignment."

PRINT "Touch any key to continue"

DO

LOOP UNTIL INKEY\$ <> "'"

GOTO 180

END IF

PRINT \#1, "Variable X8 is Site/Facility Socio-Economic Conditions"

PRINT \#1, USING a3\$; "X_8 values are "; X8V(1); " "; X8V(2); " "; X8V(3)

PRINT \#1, USING a3\$; "X_8 weights are "; X8(1); " "; X8(2); " "; X8(3)

PRINT \#1,

RETURN

210 CLS

PRINT "Material Surveillance " 


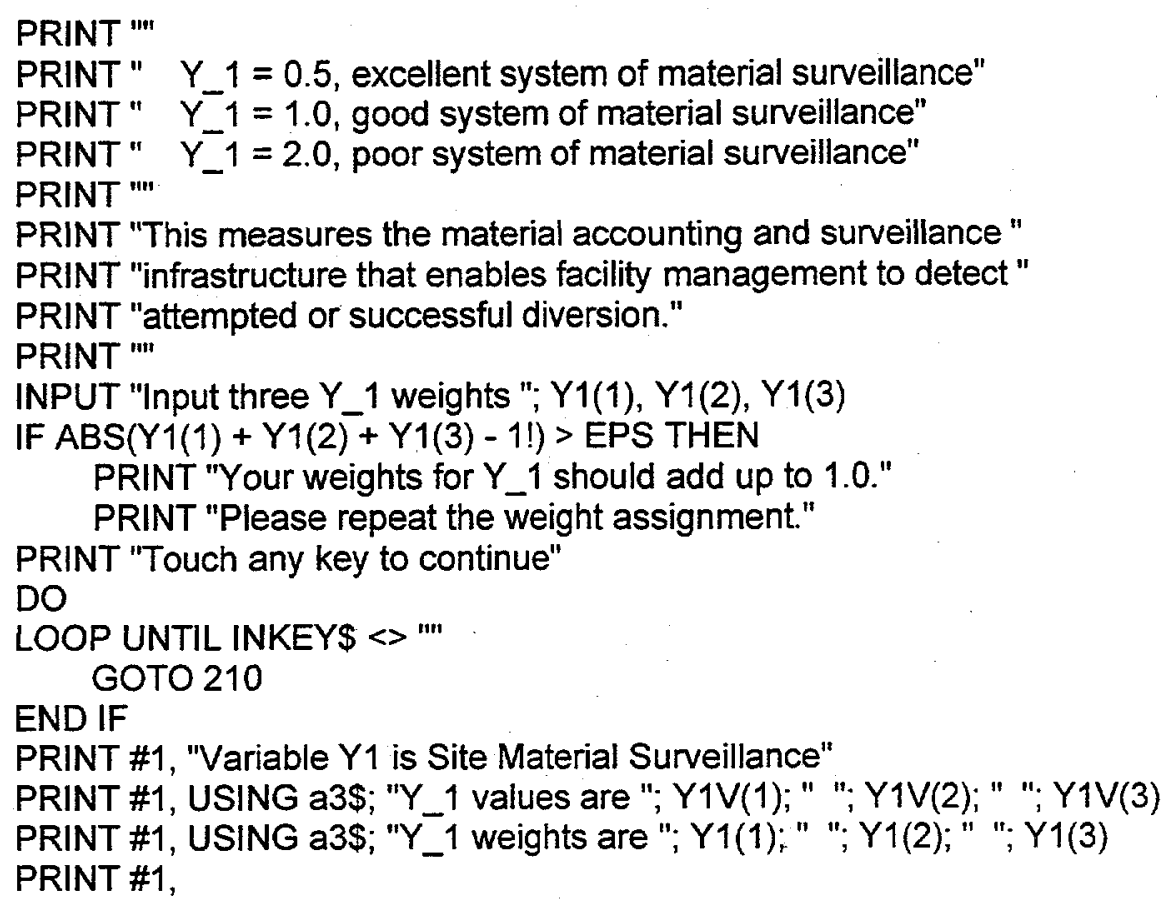

\section{RETURN}

220 CLS

PRINT "Transfer Controls "

PRINT "'!

PRINT " $Y_{2} 2=0.5$, excellent system of transfer control"

PRINT " Y_2 $=1.0$, good system of transfer control"

PRINT " $Y_{2} 2=2.0$, poor system of transfer control"

PRINT '"'

PRINT "This measures the security of material in transit from one "

PRINT "storage/utilization location to another."

PRINT "m!

INPUT "Input three $Y_{-} 2$ weights "; Y2(1), Y2(2), Y2(3)

IF ABS $(Y 2(1)+Y 2(2)+Y 2(3)-1$ !) $>$ EPS THEN

PRINT "Your weights for $Y \_2$ should add up to 1.0."

PRINT "Please repeat the weight assignment."

PRINT "Touch any key to continue"

DO

LOOP UNTIL INKEY\$ <> "'"

END IF GOTO 220

PRINT \#1, "Variable Y2 is Site Transfer Controls"

PRINT \#1, USING a3\$; "Y_2 values are "; Y2V(1); " "; Y2V(2); " "; Y2V(3)

PRINT \#1, USING a3\$; "Y_2 weights are "; Y2(1); " "; Y2(2); " "; Y2(3)

PRINT \#1,

\section{RETURN}

230 CLS 


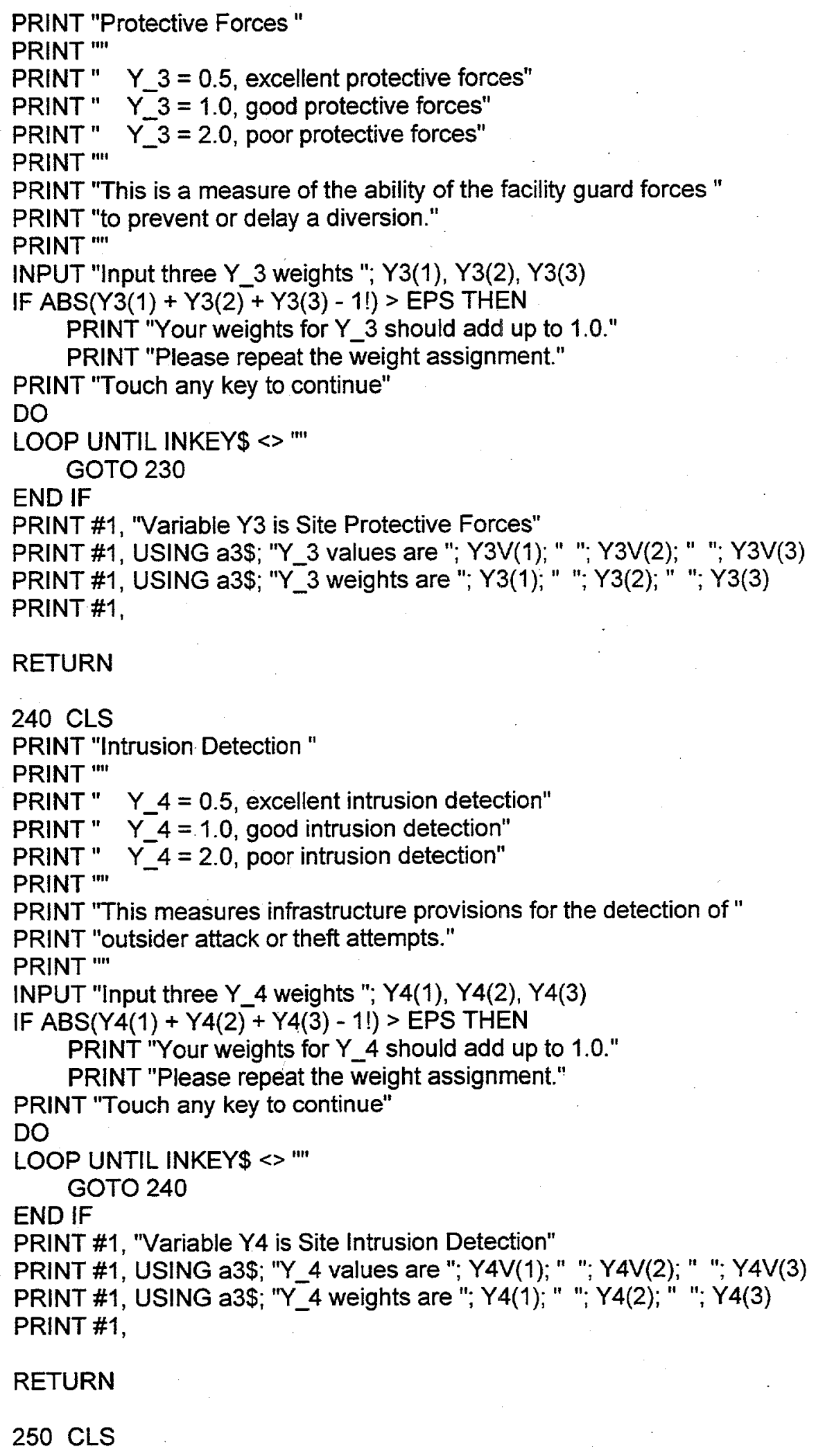




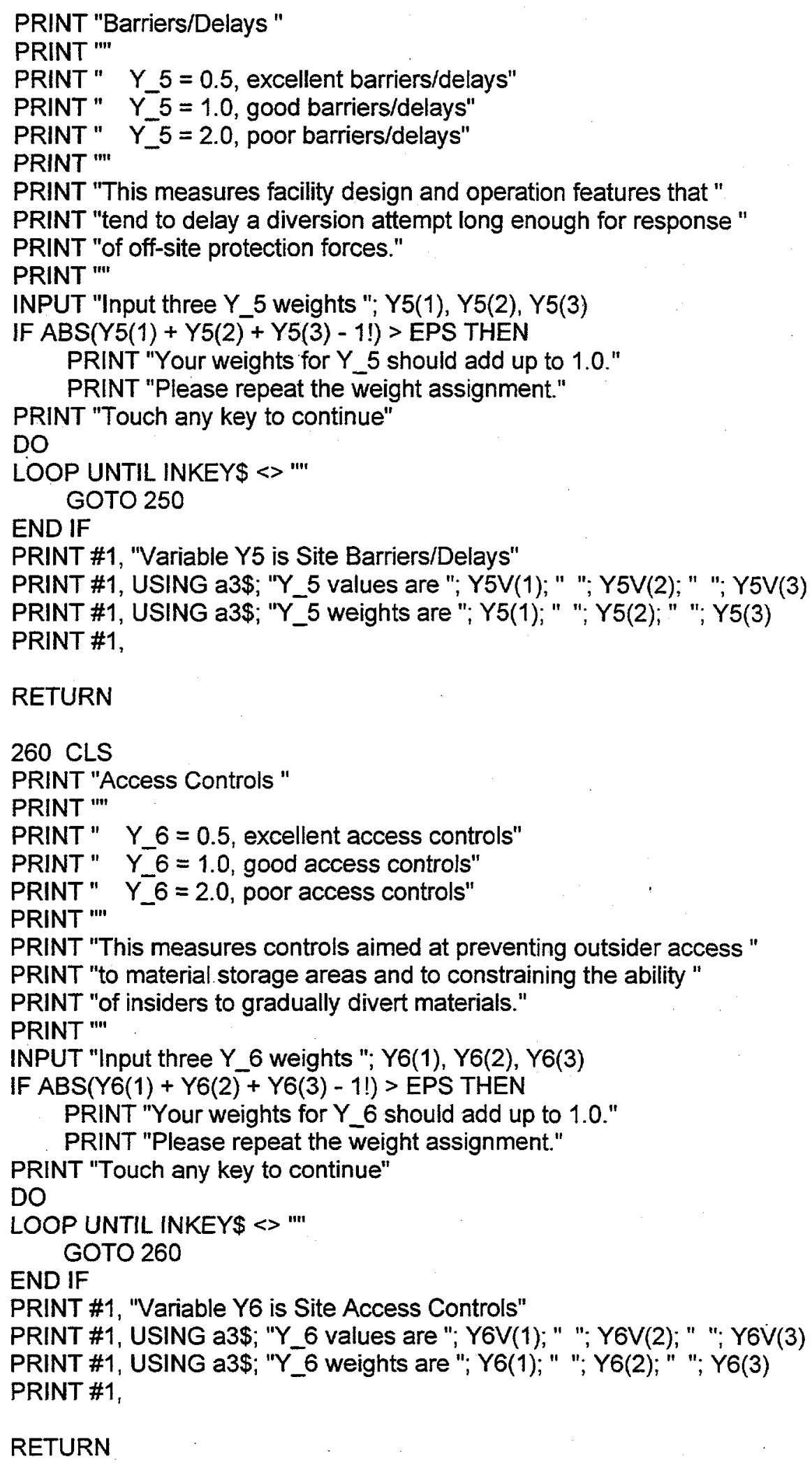


270 CLS

PRINT "Site Location "

PRINT "'"

PRINT " $\quad Y_{-} 7=0.5$, site with none or few good escape routes"

PRINT " $\quad Y_{-}^{-7}=1.5$, site with many good escape paths"

PRINT "'"

PRINT "This measures the ability of group or individual that "

PRINT "has successfully diverted material to successfully remove "

PRINT "it to a secure location and/or transfer it to the end-user. "

PRINT "'!

INPUT "Input two $Y \_7$ weights "; $Y 7(1), Y 7(2)$

IF ABS(Y7(1) + Y $7(\overline{2})-1$ !) > EPS THEN

PRINT "Your weights for $Y_{-} 7$ should add up to 1.0."

PRINT "Please repeat the weight assignment."

PRINT "Touch any key to continue"

DO

LOOP UNTIL INKEY\$ <> "'"

GOTO 270

END IF

PRINT \#1, "Variable Y7 is Site Location (as it affects interdiction of "

PRINT \#1, " diverted materials)"

PRINT \#1, USING a2\$; "Y_7 values are "; Y7V(1); " "; Y7V(2)

PRINT \#1, USING a2\$; "Y_7 weights are "; Y7(1); " "; Y7(2)

PRINT \#1,

\section{RETURN}

280. CLS

PRINT "Emergency Response "

PRINT "'"'

PRINT " $\quad Y_{8} 8=0.5$, site with excellent emergency response resources"

PRINT " Y_8 $=1.0$, site with some emergency response"

PRINT " $Y_{-} 8=2.0$, site with none or poor emergency response"

PRINT "'!

PRINT "This measures the ability of off-site emergency response to "

PRINT "interdict a forcible diversion in progress, given timely "

PRINT "notification. Note that measures $Y_{-} 4, Y_{-} 5$, and $Y_{-} 7$ also "

PRINT "condition the likelihood of successful interdiction of $a$ ".

PRINT "diversion in progress."

PRINT '"'

INPUT "Input three $Y \_8$ weights "; $Y 8(1), Y 8(2), Y 8(3)$

IF ABS(Y8(1) + Y8(2) + Y8(3) - 1!) > EPS THEN

PRINT "Your weights for $Y_{-} 8$ should add up to 1.0."

PRINT "Please repeat the weight assignment."

PRINT "Touch any key to continue"

DO

LOOP UNTIL INKEY\$ <> "'!

END IF

GOTO 280

PRINT \#1, "Variable Y8 is Site Emergency Response (as it affects "

PRINT \#1, " interdiction of diverted materials)" 
PRINT \#1, USING a3\$; "Y_8 values are "; Y8V(1); " "; Y8V(2); " "; Y8V(3)

PRINT \#1, USING a3\$; "Y_8 weights are "; Y8(1); " "; Y8(2); " "; Y8(3)

PRINT \#1,

\section{RETURN}

285 CLS

PRINT "Overall Capability of Host Country Safeguards Measures"

PRINT "'"

PRINT " Y_9 $=0.5$, Robust safeguards measures "

PRINT " Y_9 $=1.0$, Safeguards with some weaknesses in design "

PRINT " or implementation "

PRINT " Y_9 $=2.0$, Weak or non-existent safeguards "

PRINT "'

PRINT "This measures the overall capability of safeguards measures "

PRINT "to detect/alarm attempted state transfer of weapons material "

PRINT "to unauthorized state- or sub-state-organizations."

PRINT "'!

INPUT "Input three $Y$ 9 weights "; $Y 9(1), Y 9(2), Y 9(3)$

IF ABS(Y9(1) + Y9(2) + Y9(3) - 1!) > EPS THEN

PRINT "Your weights for $Y_{-}$9 should add up to 1.0."

PRINT "Please repeat the weight assignment."

PRINT "Touch any key to continue"

DO

LOOP UNTIL INKEY\$ <> "'!

GOTO 285

END IF

PRINT \#1, "Variable Y9 is Overall Capability of Host Country Safeguards"

PRINT \#1, USING a3\$; "Y_9 values are "; Y9V(1); " "; Y9V(2); " "; Y9V(3)

PRINT \#1, USING a3\$; "Y_9 weights are "; Y9(1); " "; Y9(2); " "; Y9(3)

PRINT \#1,

\section{RETURN}

290 CLS

PRINT "Socio-political stability "

PRINT '"!'

PRINT " Y_10 $=0.5$, stable government with good foreign "

PRINT " $\quad$ relations, checks and balances"

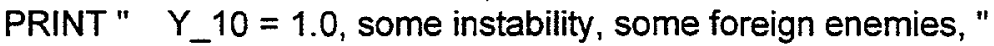

PRINT " - some checks and balances"

PRINT " Y Y $10=2.0$, significant instability, many factions, "

PRINT " - many enemies, no checks and balances"

PRINT 'm!

PRINT "This attempts to measure the likelihood that all or part of "

PRINT "the relevant government organizations will formulate and "

PRINT "implement or will fail to impede a state transfer diversion"

PRINT "attempt. "

PRINT "'!"

INPUT "Input three $Y$ 10 weights "; $Y 10(1), Y 10(2), Y 10(3)$

IF ABS $(Y 10(1)+Y 10(2)+Y 10(3)-1$ !) > EPS THEN

PRINT "Your weights for $Y_{-} 10$ should add up to 1.0." 
PRINT "Please repeat the weight assignment."

PRINT "Touch any key to continue"

DO

LOOP UNTIL INKEY\$ <> "'!

GOTO 290

END IF

PRINT \#1, "Variable Y10 is Host Country Socio-political Stability"

PRINT \#1, USING a3\$; "Y_10 values are "; Y10V(1); " "; Y10V(2); " "; Y10V(3)

PRINT \#1, USING a3\$; "Y_10 weights are "; Y10(1); " "; Y10(2); " "; Y10(3)

PRINT \#1,

\section{RETURN}

- Subroutine 1000 calculates Threat and Vulnerability Probabilities

- for the Insider Theft scenario type

1000 GOSUB 100

GOSUB 140

GOSUB 150

GOSUB 170

GOSUB 180

GOSUB 210

GOSUB 220

DIM ITTHREATV! $(2,4,2,3)$

DIM ITTHREATP! $(2,4,2,3)$

DIM ITVULNV! $(3,3)$

DIM ITVULNP! $(3,3)$

FORI $=1$ TO 2

FOR $\mathrm{J}=1 \mathrm{TO} 4$

FOR K $=1$ TO 2

FOR L $=1$ TO 3

ITTHREATV! $(\mathrm{I}, \mathrm{J}, \mathrm{K}, \mathrm{L})=\mathrm{X} 4 \mathrm{~V}(\mathrm{I}) * \times 5 \mathrm{~V}(\mathrm{~J}){ }^{*} \times 7 \mathrm{~V}(\mathrm{~K}){ }^{*} \mathrm{X8V}(\mathrm{L})$

ITTHREATP! $(\mathrm{I}, \mathrm{J}, \mathrm{K}, \mathrm{L})=\mathrm{X} 4(\mathrm{l}){ }^{*} \mathrm{X} 5(\mathrm{~J}){ }^{*} \mathrm{X} 7(\mathrm{~K}){ }^{*} \mathrm{X} 8(\mathrm{~L})$

ITTHREATPROB!((ITTHREATV! $(I, J, K, L)$ * 10) + 1) = ITTHREATPROB!((ITTHREATV! $(I, J, K, L)$ * 10) + 1) + ITTHREATP! (I, J, K, L)

' PRINT \#1, "ITTHREATV!(-,-,-,"; I; ","; J; ",-,"; L; ","; L; ") = "; ITTHREATV!(I, J, K, L)

' PRINT \#1, "ITTHREATP!(-,-,-,; ; ; ",,"; J; ",-,"; L; ","; L; ") = "; ITTHREATP!(I, J, K, L)

'PRINT \#1,

NEXT L

NEXT K

NEXT J

NEXT I

FOR I = 1 TO 3

FOR $J=1$ TO 3

ITVULNV! $(I, J)=Y 1 V(I) * Y 2 V(J)$

ITVULNP! $(I, J)=Y 1(I) * Y 2(J)$

ITVULNPROB!((ITVULNV!(I, J) * 25) + 1) = ITVULNPROB!(ITVULNV!(I, J) * 25) + 1) + ITVULNP!(I, J)

- PRINT \#1, "ITVULNV!("; I; ","; J; ") = "; ITVULNV!(I, J)

- PRINT \#1, "ITVULNP!("; I; ","; J; ") = "; ITVULNP!(I, J)

- PRINT \#1, NEXT J

NEXT I 
' $F O R I=1$ TO 101

' PRINT \#1, USING tv\$; "For Threat value = "; $(I-1) / 10$; " the probability is "; ITTHREATPROB!(I)

' NEXT I

PRINT \#1,

' FOR I = 1 TO 101

' PRINT \#1, USING tv\$; "For Vulnerability value = "; $(1-1) / 25$; " the probability is "; ITVULNPROB!(I)

' NEXT I

GOSUB 5000

GOSUB 5100

GOSUB 5200

RETURN

- Subroutine 5000 accumulates the Threat probabilities values into

' four Threat category probability values for the Insider Theft

- scenario type; it also accumulates Vulnerability probabilities into

- Vulnerability category probabilities for the Insider Theft scenario

' type.

5000 FOR I $=1$ TO 3

NEXT I

THREAT $(1)=$ THREAT $(1)+$ ITTHREATPROB! $(1)$

FOR I $=4$ TO 9

NEXT I

THREAT(2) = THREAT(2) + ITTHREATPROB!(1)

FOR $1=10$ TO 30

THREAT(3) $=$ THREAT $(3)+$ ITTHREATPROB! $(1)$

NEXT I

FOR I = 31 TO 101

NEXT 1

THREAT(4) = THREAT $(4)+$ ITTHREATPROB! $(1)$

print \#1,

PRINT \#1, USING c\$; "For Threat category Negligible, the probability is ";THREAT(1)

PRINT \#1, USING c\$; "For Threat category Low, the probability is ";THREAT(2)

PRINT \#1, USING c\$; "For Threat category High, the probability is ";THREAT(3)

PRINT \#1, USING c\$; "For Threat category Very High, the probability is ";THREAT(4)

print \#1,

FOR I $=1$ TO 10

NEXT I

VULN $(1)=$ VULN $(1)+$ ITVULNPROB! $(1)$

FOR I = 11 TO 22

NEXT I

$\operatorname{VULN}(2)=\operatorname{VULN}(2)+$ ITVULNPROB!(I)

FOR I $=23$ TO 40

NEXT I

VULN $(3)=\operatorname{VULN}(3)+$ ITVULNPROB! $(1)$

FOR I $=41$ TO 101

NEXT I

VULN(4) $=$ VULN(4) + ITVULNPROB!(I)

print \#1,

PRINT \#1, USING c\$; "For Vulnerability category Negligible, the probability is ";VULN(1)

D.15 
PRINT \#1, USING $c \$$; "For Vulnerability category Low, the probability is ";VULN(2) PRINT \#1, USING c\$; "For Vulnerability category High, the probability is ";VULN(3) PRINT \#1, USING C\$; "For Vulnerability category Very High, the probability is ";VULN(4) print \#1,

RETURN

- Subroutine 5100 computes the Likelihood matrix probability values and - accumulates them into the five Likelihood category probabilities

5100 FORI $=1$ TO 4

FOR $J=1$ TO 4

LMAT! $(\mathrm{I}, \mathrm{J})=$ THREAT $(\mathrm{l})$ * VULN $(\mathrm{J})$

NEXT I NEXT J

LHOOD! $(1)=$ LMAT! $(1,1)+\operatorname{LMAT}(1,2)+\operatorname{LMAT} !(1,3)+\operatorname{LMAT} !(2,1)$

LHOOD! $(2)=$ LMAT! $(1,4)+\operatorname{LMAT} !(2,2)+\operatorname{LMAT} !(3,1)+\operatorname{LMAT} !(4,1)$

LHOOD! $(3)=$ LMAT! $(2,3)+\operatorname{LMAT} !(3,2)+\operatorname{LMAT} !(4,2)$

LHOOD! $(4)=\operatorname{LMAT} !(2,4)+\operatorname{LMAT} !(3,3)+\operatorname{LMAT} !(4,3)$

LHOOD! $(5)=\operatorname{LMAT!}(3,4)+\operatorname{LMAT} !(4,4)$

PRINT \#1,

PRINT \#1, USING c\$; "Probability for Likelihood category Negligible = "; LHOOD!(1)

PRINT \#1, USING c\$; "Probability for Likelihood category Low = "; LHOOD!(2)

PRINT \#1, USING C\$; "Probability for Likelihood category Medium = "; LHOOD!(3)

PRINT \#1, USING c\$; "Probability for Likelihood category High = "; LHOOD!(4)

PRINT \#1, USING c\$; "Probability for Likelihood category Very High = "; LHOOD!(5)

\section{RETURN}

- Subroutine 5200 solicits input from the evaluator for the Consequence

- measure, given as weights/probabilities assigned to each of the five

- Consequence categories. This input is echoed to output. Finally,

- Risk Matrix probabilities are calculated and then accumulated into

' the five Risk category probabilities

5200 CLS

PRINT "Enter estimate of sitelfacility diversion consequences."

PRINT "Enter five weights/probabilities associated with the categories:"

PRINT " Negligible = Fission products or other non-weapons material"

PRINT " (IAEA Attractiveness Level E or less)"

PRINT "'

PRINT " Low = Small amounts of difficult to convert materials"

PRINT " - $\quad$ (IAEA Attractiveness Level D, Category IV)"

PRINT '”'

PRINT"

PRINT "

PRINT "

PRINT "

PRINT "'"

PRINT "

print"

Medium = Small amounts of easily convertible or large amounts"

of difficult to convert materials"

(IAEA Attractiveness Level D, Categories I,II, or III"

and Attractiveness Levels B and C, Category IV)"

PRINT " $\quad$ (IAEA Attractiveness Level B and C, " 


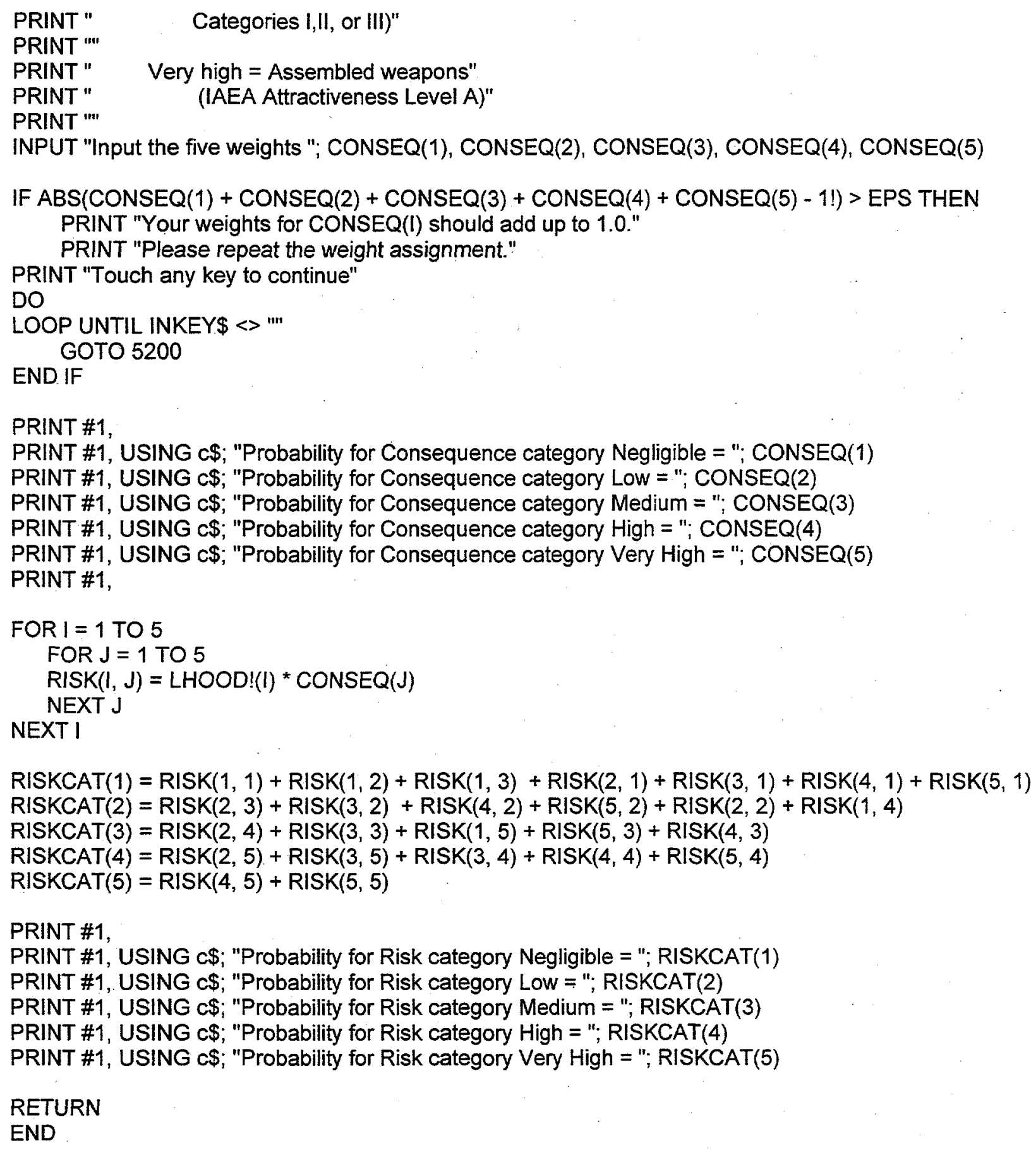




\section{Source code for the Outsider Attack Scenario}

\footnotetext{
This is a prototype, with a text-based user interface. It implements the proposed threat/vulnerability measure aggregation scheme, including handling of evaluator uncertainty

The program will begin displaying the relevant threat and vulnerability sub-measures one by one and prompting the evaluator for his/her estimates of the weights (or probabilities) to be assigned to each of the possible sub-measure values. For each sub-measure, these weights should sum to 1 . The program will solicit weights for the consequence measure, which is based on the quantity and attractiveness of the nuclear materials available for diversion at the site.

When the input for all relevant sub-measures has been entered, the 1 program will compute the aggregate values, will assign categorical 1 values to the threat and vulnerability measures, and will compute the ' corresponding diversion risk measure. The evaluator-assigned

' uncertainties will be propagated all the way through to the ' diversion risk measure.
}

DEFDBL A-H, O-Z

DEFINT I-N

OPTION BASE 1

EPS $=.001$
DIM X1(3) ' Blood feud - WEIGHTS
DIM X2(2) ' Political/ideological motivations -- WEIGHTS
DIM X3(3) 'Religious motivations -- WEIGHTS
DIM X4(2) 'Perception of target vulnerability - WEIGHTS
DIM X5(4) ' Availability of resources - WEIGHTS
DIM X6(3) ' Training and leadership -- WEIGHTS
DIM X7(2) ' Knowledge and intelligence - WEIGHTS
DIM X8(3) ' Site/facility socio-economic conditions -- WEIGHTS
DIM Y1(3) ' Material surveillance -- WEIGHTS
DIM Y2(3) ' Transfer controls - WEIGHTS
DIM Y3(3) '. Protective forces -- WEIGHTS
DIM Y4(3) ' Intrusion detection -- WEIGHTS
DIM Y5(3) ' Barriers/delays -- WEIGHTS
DIM Y6(3) ' Access controls - WEIGHTS 


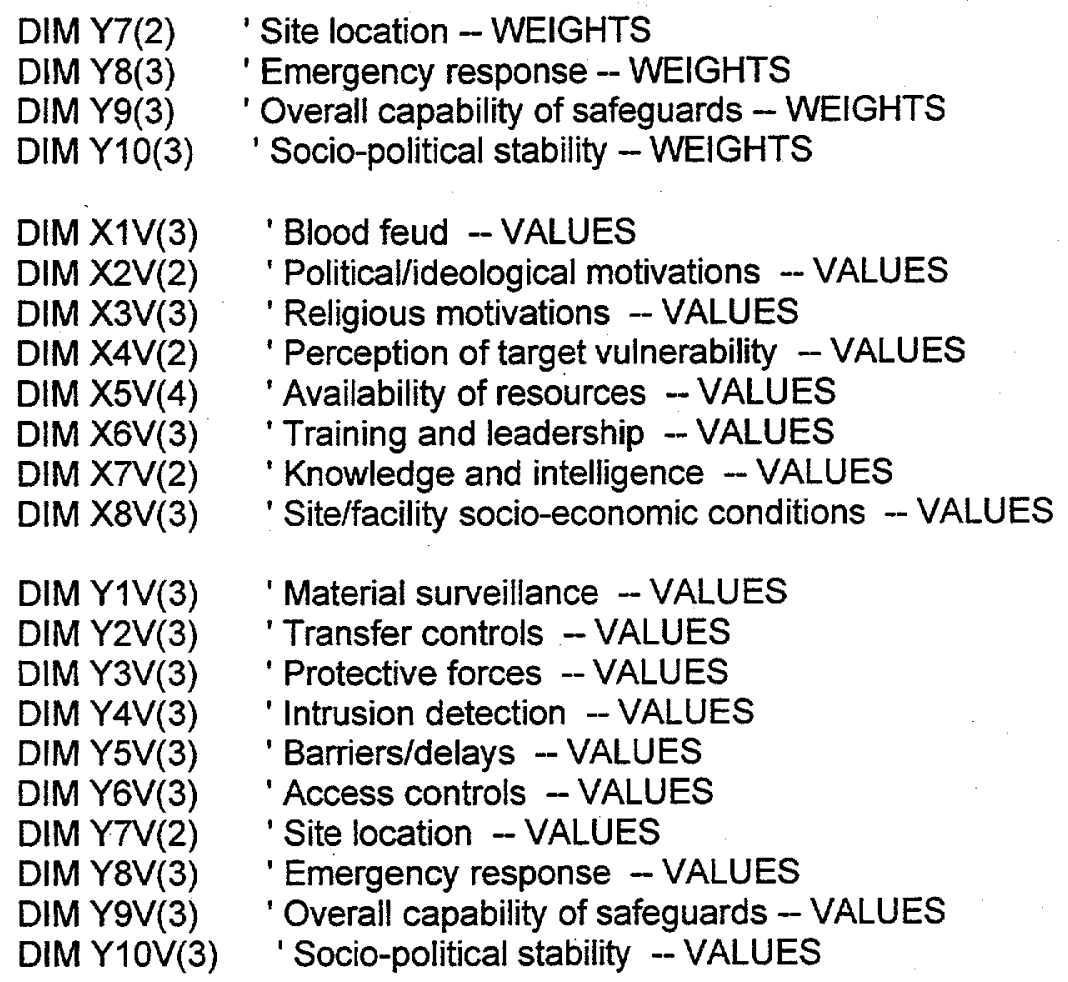


DATA $0.5,1.0,2.0$

FOR I = 1 TO 2: READ YTV(I): NEXT I

DATA $0.5,1.5$

FOR I = 1 TO 3: READ Y8V(I): NEXT I

DATA $0.5,1.0,2.0$

FOR I = 1 TO 3: READ Y9V(I): NEXT I

DATA $0.5,1.0,2.0$

FOR I = 1 TO 3: READ Y10V(I): NEXT I

DATA $0.5,1.0,2.0$

DIM OATHREATPROB!(101)

DIM OAVULNPROB!(101)

DIM THREAT(4) 'Threat category probabilities

DIM VULN(4) Vulnerability category probabilities

DIM LHOOD!(5) 'Likelihood category probabilities

DIM LMAT! $(4,4)$ 'Likelihood matrix probabilities

DIM CONSEQ(5) 'Consequence category probabilities

DIM RISK $(5,5)$ 'Risk matrix probabilities

DIM RISKCAT(5) 'Risk category probabilities

DIM scentype\$(4) ' Scenario type names

FOR I = 1 TO 4: READ scentype\$(I): NEXT I

DATA "Insider theft", "Outsider theft", "Outsider attack", "State transfer"

' Define printout formats

a2\$ = "\&\#.\#\# \&\#.\#"

a3\$ $=" \& \# . \#$ \&\#.\# \&\#.\#"

a4\$ $=$ "\&\#.\#\#\&\#.\# \&\#.\#\#\&\#.\#"

tv\$ $=$ "\& \#\#\# \&\#.\#"

$\mathrm{C} \$=$ "\&\#.\#"

$1 c \$=" \& \# \& \#$ \#\#"

debug1\$="\&\# \&\#\&\#\&\#\&\#\&\#\&\#\#\#"

debug2\$ = "\&\#.\#\#.\#\#.\#\#.\#"

INPUT "Enter name of output file "; outfile\$

OPEN outfile\$ FOR OUTPUT AS \#1

INPUT "Enter site/facility name "; sitename\$

PRINT \#1, "Evaluation of site/facility: "; sitename\$

PRINT \#1, "For scenario type: "; scentype\$(3)

PRINT \#1, "Output file name: $\quad$ "; outfile\$

PRINT \#1, "Program and Version: Version 4 of OA_EVAL"

PRINT \#1,

GOSUB 3000

STOP 
' Subroutines 110 through 290 solicit evaluator input for Threat and

' Vulnerability sub-measures. Subroutine 100 explains that the Threat ' and Vulnerability sub-measures are all treated as random variables, ' requiring that probabilities (or weights) summing to 1 must be assigned ' to each of the numerical values taken on by that submeasure.

100 CLS

PRINT "The Threat and Vulnerability sub-measures used in this scheme"

PRINT "for estimating the risk of diversion of nuclear materials are"

PRINT "all treated as random variables, requiring that probabilities"

PRINT "(or weights) summing to 1 must be assigned to each of the"

PRINT "numerical values taken on by that submeasure. The input routines"

PRINT "will enforce this requirement."

PRINT "'!

PRINT "In addition, the assignment of probabilities to the sub-measure"

PRINT "can be used to represent the evaluators uncertainty about the"

PRINT "correct value of the sub-measure. For instance, in the absence of"

PRINT "any information about the true value of the sub-measure"

PRINT "(i.e., complete uncertainty), the evaluator should assign equal"

PRINT "weights to all of the sub-measure values."

PRINT "'!

PRINT "Touch any key to continue"

DO

LOOP UNTIL INKEY\$ <> "'

RETURN

110 CLS

PRINT "Existence of a Long Standing Conflict "

PRINT "'"

PRINT " X_1 $=0.5$, no underlying conflict"

PRINT " $\quad X \_1=1.0$, recently developed conflict (decades-long duration)"

PRINT " $X_{-}^{-1}=2.0$, long standing conflict (centuries-long duration)"

PRINT "'!

PRINT "*Long Standing Conflict* is shorthand for aspects of intensity "

PRINT "and long duration in the motivation of the threat groups. It can "

PRINT "derive from extreme religious prejudice, language/cultural "

PRINT "differences, and/or from a long history of oppression,"

PRINT "suppression, or warfare between two groups of people. Examples "

PRINT "include Catholic Irish vs. Irish Protestants and the British"

PRINT "or the Basques vs. the Spanish or Islamic fundamentals vs. "

PRINT "secular Moslem governments. "

PRINT '"'

INPUT "Input three X_1 weights "; X1(1), X1(2), X1(3)

IF ABS $(X 1(1)+X 1(2)+X 1(3)-1 !)>$ EPS THEN

PRINT "Your weights for $X \_1$ should add up to $1.0 . "$

PRINT "Please repeat the weight assignment."

PRINT "Touch any key to continue"

DO

LOOP UNTIL INKEY\$ $<>$ "'"

GOTO 110 
END IF

PRINT \#1, "Variable $X 1$ is Existence of a Long Standing Conflict"

PRINT \#1, USING a3\$; "X_1 values are "; X1V(1); " "; X1V(2); " "; X1V(3)

PRINT \#1, USING a3\$; "X_1 weights are "; X1(1); " "; X1(2); " "; X1(3)

PRINT \#1,

\section{RETURN}

$120 \mathrm{CLS}$

PRINT "Political//deological Motivations "

PRINT "'"

PRINT " X_2 $=0.5$, no political/ideological basis "

PRINT " $\quad X_{-} 2=1.0$, political/ideological motivations"

PRINT "'!

PRINT "Examples would be support for terrorism as a component "

PRINT "of the cold war struggle between Communism and the West."

PRINT "'!

INPUT "Input two X_2 weights "; $X 2(1), X 2(2)$

IF ABS $(X 2(1)+X 2(\overline{2})-1$ !) $>$ EPS THEN

PRINT "Your weights for $X \_2$ should add up to 1.0."

PRINT "Please repeat the weight assignment."

PRINT "Touch any key to continue"

DO

LOOP UNTIL INKEY\$ <> "'"

GOTO 120

END IF

PRINT \#1, "Variable X2 is Political//deological Motivations"

PRINT \#1, USING a2\$; "X_2 values are "; X2V(1); " "; X2V(2)

PRINT \#1, USING a2\$; "X_2 weights are "; X2(1); " "; X2(2)

PRINT \#1,

\section{RETURN}

130 CLS

PRINT "Religious Motivations "

PRINT " "

PRINT " $\quad X 3=0.5$, no religious animosity"

PRINT " $\quad X-3=1.0$, some religious animosity"

PRINT " $\quad X_{3} 3=2.0$, strong religious animosity"

PRINT "'"

PRINT "The intent here is to capture aspects of motivation distinct "

PRINT "from possible religious components of the "; blood; feud; " measure "

PRINT "above."

PRINT "*!

INPUT "Input three $X \_3$ weights "; $X 3(1), X 3(2), X 3(3)$

IF ABS(X3(1) + X3(2) + X3(3) - 1!) > EPS THEN

PRINT "Your weights for $X \_3$ should add up to 1.0."

PRINT "Please repeat the weight assignment."

PRINT "Touch any key to continue"

DO

LOOP UNTIL INKEY\$ $<>$ "'!

GOTO 130 
END IF

PRINT \#1, "Variable X3 is Religious Motivations"

PRINT \#1, USING a3\$; "X_3 values are "; X3V(1); " "; X3V(2); " "; X3V(3)

PRINT \#1, USING a3\$; "X_3 weights are "; X3(1); " "; X3(2); " "; X3(3)

PRINT \#1,

\section{RETURN}

140 CLS

PRINT "Perception of target vulnerability "

PRINT "'"

PRINT " $X 44=0.5$, facility not perceived as vulnerable "

PRINT " X_4 $=1.5$, facility perceived as vulnerable"

PRINT "'"

PRINT "The reasoning here is that, even for a threat group predisposed "

PRINT "to action, if a particular facility is perceived as not vulnerable, "

PRINT "they will direct their attention elsewhere."

PRINT "'!

INPUT "Input two $X 44$ weights "; $X 4(1), X 4(2)$

IF ABS $(X 4(1)+X 4(\overline{2})-1$ !) $>$ EPS THEN

PRINT "Your weights for $X \_4$ should add up to 1.0."

PRINT "Please repeat the weight assignment."

PRINT "Touch any key to continue"

DO

LOOP UNTIL INKEY\$ <> "'"

GOTO 140

END IF

PRINT \#1, "Variable X4 is Perception of Target Vulnerability"

PRINT \#1, USING a2\$; "X_4 values are "; X4V(1); " "; X4V(2)

PRINT \#1, USING a2\$; "X_4 weights are "; $X 4(1)$; " "; $X 4(2)$.

PRINT \#1,

\section{RETURN}

150 CLS

PRINT "Availability of resources "

PRINT "'!

PRINT " X_5 $=0.0$, no resources available"

PRINT " $X 5=0.5$, modest, locally collected resources"

PRINT " X_5 = 1.0, intermediate, state-supported"

PRINT " X_5 = 2.0, large, state-supported"

PRINT '"'

PRINT "This is a measure of the resource base of the threat group."

PRINT "'!

INPUT "Input four $X 5$ weights "; X5(1), X5(2), X5(3), X5(4)

IF ABS $(X 5(1)+X 5(\overline{2})+X 5(3)+X 5(4)-1$ !) $>$ EPS THEN

PRINT "Your weights for $X \_5$ should add up to 1.0."

PRINT "Please repeat the weight assignment."

PRINT "Touch any key to continue"

DO

LOOP UNTIL INKEY\$ <> "'!

GOTO 150 
END IF

PRINT \#1, "Variable X5 is Availability of Threat Group Resources"

PRINT \#1, USING a4\$; "X_5 values are "; X5V(1);" "; X5V(2); " "; X5V(3); " "; X5V(4)

PRINT \#1, USING a4\$; "X_5 weights are "; X5(1); " "; X5(2); " "; X5(3); " "; X5(4)

PRINT \#1,

\section{RETURN}

160 CLS

PRINT "Training and leadership "

PRINT "'"

PRINT " X_6 $=0.5$, poor training and leadership"

PRINT " $X-6=1.0$, intermediate training and leadership"

PRINT " X_6 $=2.0$, well-trained and led"

PRINT "'!

PRINT "This is a measure of the quality of training and leadership "

PRINT "of the threat organization."

PRINT "'"

INPUT "Input three X_6 weights "; X6(1), X6(2), X6(3)

IF ABS $(X 6(1)+X 6(2)+X 6(3)-1 !)>$ EPS THEN

PRINT "Your weights for $X \_6$ should add up to 1.0."

PRINT "Please repeat the weight assignment."

PRINT "Touch any key to continue"

DO

LOOP UNTIL INKEY\$ <> "'!

GOTO 160

END IF

PRINT \#1, "Variable X6 is Threat Group Training and Leadership"

PRINT \#1, USING a3\$; "X_6 values are "; X6V(1); " "; X6V(2); " "; X6V(3)

PRINT \#1, USING a3\$; "X_6 weights are "; X6(1); " "; X6(2); " "; X6(3)

PRINT \#1,

\section{RETURN}

170 CLS

PRINT "Knowledge and intelligence "

PRINT "'"

PRINT " $\quad X \_7=0.5$, poor intelligence capabilities"

PRINT " $X \_7=2.0$, good intelligence capabilities"

PRINT "'

PRINT "This is a measure of the access of the threat organization "

PRINT "to information about the potential target and their ability "

PRINT "to use that information."

PRINT '"!

INPUT "Input two $X \_7$ weights "; $X 7(1), X 7(2)$

IF ABS $(X 7(1)+X 7(\overline{2})-1$ !) > EPS THEN

PRINT "Your weights for $X \_7$ should add up to 1.0."

PRINT "Please repeat the weight assignment."

PRINT "Touch any key to continue"

DO

LOOP UNTIL INKEY\$ <> "'"

GOTO 170 
END IF

PRINT \#1, "Variable X7 is Threat Group Knowledge and Intelligence Capability"

PRINT \#1, USING a2\$; "X 7 values are "; X7V(1);" "; X7V(2)

PRINT \#1, USING a2\$; "X_7 weights are "; $X 7(1)$; " "; $X 7(2)$

PRINT \#1

\section{RETURN}

180 CLS

PRINT "Site/facility socio-economic conditions "

PRINT "'!

PRINT ". X_8 $=0.75$, good conditions"

PRINT " X_8 $=1.0$, not-so-good"

PRINT " X_8 $=1.5$, extremely difficult conditions"

PRINT "'

PRINT "This attempts to measure the likelihood that the threat "

PRINT "organization can subvert/coopt insiders to support its"

PRINT "diversion attempt or that insiders will themselves attempt diversion."

PRINT "'!

INPUT "Input three $X \_8$ weights "; $X 8(1), X 8(2), X 8(3)$

IF ABS(X8(1) + X8(2) + X8(3) - 1!) > EPS THEN

PRINT "Your weights for $X \_8$ should add up to 1.0."

PRINT "Please repeat the weight assignment."

PRINT "Touch any key to continue"

DO

LOOP UNTIL INKEY\$ <> "'"

END IF

GOTO 180

PRINT \#1, "Variable X8 is Site/Facility Socio-Economic Conditions"

PRINT \#1, USING a3\$; "X_8 values are "; X8V(1); " "; X8V(2); " "; X8V(3)

PRINT \#1, USING a3\$; "X_8 weights are "; X8(1); " "; $X 8(2)$; " "; $X 8(3)$

PRINT \#1,

RETURN

210 CLS

PRINT "Material Surveillance "

PRINT "'!'

PRINT " $Y_{-} 1=0.5$, excellent system of material surveillance"

PRINT " $Y 1=1.0$, good system of material surveillance"

PRINT " $Y_{-1}^{-1}=2.0$, poor system of material surveillance"

PRINT '"'

PRINT "This measures the material accounting and surveillance "

PRINT "infrastructure that enables facility management to detect "

PRINT "attempted or successful diversion."

PRINT "'"

INPUT "Input three $Y$ 1 weights "; $Y 1(1), Y 1(2), Y 1(3)$

IF ABS(Y1(1) + Y1(2) + Y1(3) - 1!) > EPS THEN

PRINT "Your weights for $Y_{-}$1 should add up to 1.0."

PRINT "Please repeat the weight assignment."

PRINT "Touch any key to continue" 
DO

LOOP UNTIL INKEY\$ <> "'"

GOTO 210

END IF

PRINT \#1, "Variable $Y 1$ is Site Material Surveillance"

PRINT \#1, USING a3\$; "Y_1 values are "; Y1V(1); " "; Y1V(2); " "; Y1V(3)

PRINT \#1, USING a3\$; "Y_1 weights are "; Y1(1); " "; Y1(2); " "; Y1(3)

PRINT \#1,

\section{RETURN}

\section{CLS}

PRINT "Transfer Controls "

PRINT "'"

PRINT " $\quad Y_{2} 2=0.5$, excellent system of transfer control"

PRINT " Y_2 = 1.0, good system of transfer control"

PRINT " $Y_{-} 2=2.0$, poor system of transfer control"

PRINT "'"

PRINT "This measures the security of material in transit from one "

PRINT "storage/utilization location to another."

PRINT "'!

INPUT "Input three Y_2 weights "; Y2(1), Y2(2), Y2(3)

IF ABS $(Y 2(1)+Y 2(2)+Y 2(3)-1$ !) $>$ EPS THEN

PRINT "Your weights for $Y \_2$ should add up to 1.0."

PRINT "Please repeat the weight assignment."

PRINT "Touch any key to continue"

DO

LOOP UNTIL INKEY\$ $<>$ "'!

GOTO 220

END IF

PRINT \#1, "Variable Y2 is Site Transfer Controls"

PRINT \#1, USING a3\$; "Y_2 values are "; Y2V(1); " "; Y2V(2); " "; Y2V(3)

PRINT \#1, USING a3\$; "Y_2 weights are "; Y2(1); " "; Y2(2); " "; Y2(3)

PRINT \#1,

\section{RETURN}

230 CLS

PRINT "Protective Forces "

PRINT "'"

PRINT " Y_3 $=0.5$, excellent protective forces"

PRINT " Y_3 = 1.0, good protective forces"

PRINT " $Y_{-}^{-3}=2.0$, poor protective forces"

PRINT "'"

PRINT "This is a measure of the ability of the facility guard forces "

PRINT "to prevent or delay a diversion."

PRINT "'

INPUT "Input three Y_3 weights "; $Y 3(1), Y 3(2), Y 3(3)$

IF ABS $(Y 3(1)+Y 3(2)+Y 3(3)-1$ !) $>$ EPS THEN

PRINT "Your weights for $Y_{-} 3$ should add up to 1.0."

PRINT "Please repeat the weight assignment."

PRINT "Touch any key to continue" 


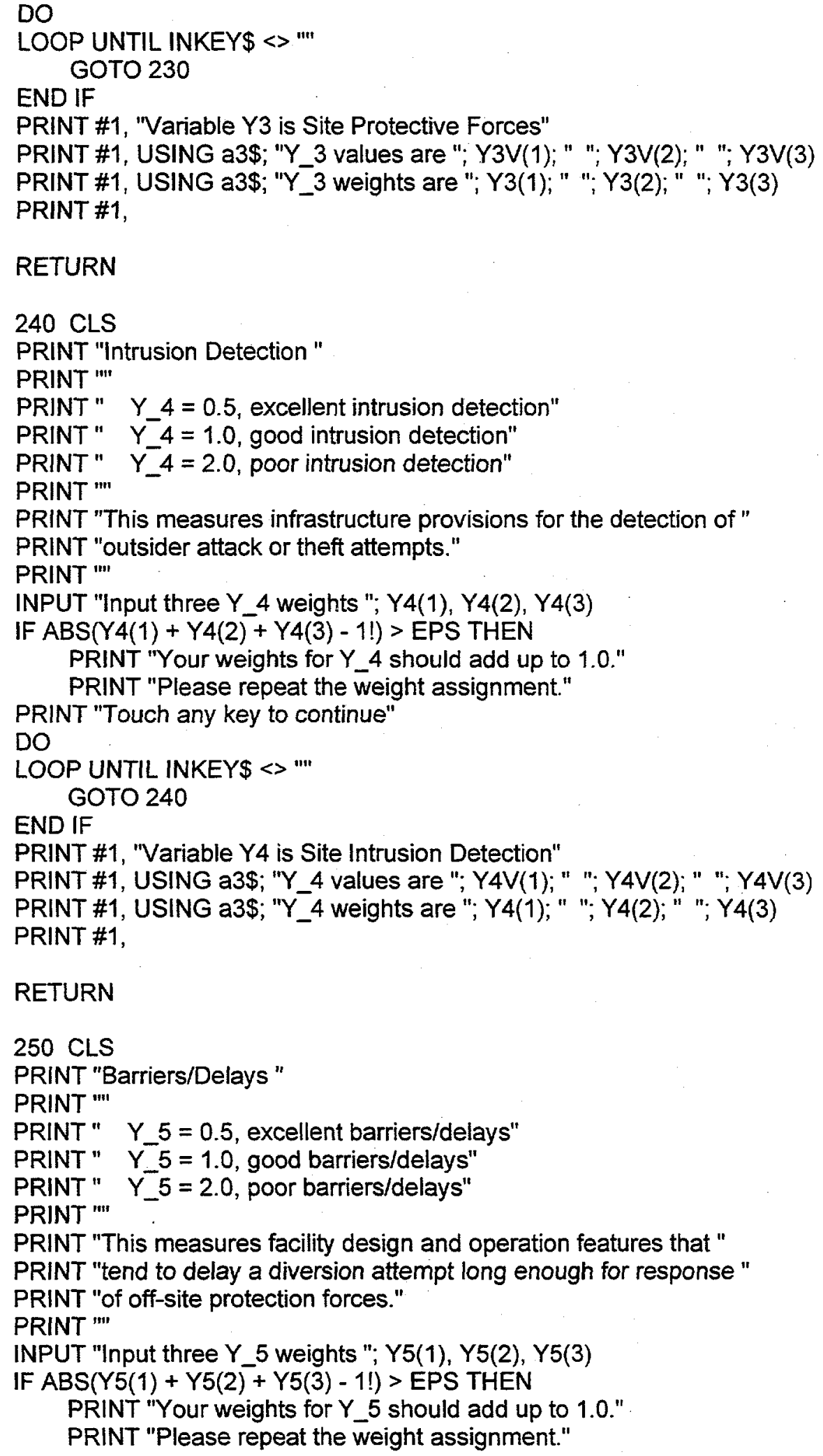


PRINT "Touch any key to continue"

DO

LOOP UNTIL INKEY\$ $<>$ "'!

GOTO 250

END IF

PRINT \#1, "Variable Y5 is Site Barriers/Delays"

PRINT \#1, USING a3\$; "Y_5 values are "; Y5V(1); " "; Y5V(2); " "; Y5V(3)

PRINT \#1, USING a3\$; "Y_5 weights are "; Y5(1); " "; Y5(2); " "; Y5(3)

PRINT \#1,

\section{RETURN}

260 CLS

PRINT "Access Controls "

PRINT "'"

PRINT " Y_6 $=0.5$, excellent access controls"

PRINT " Y $6=1.0$, good access controls"

PRINT" Y_6 $=2.0$, poor access controls"

PRINT '"'

PRINT "This measures controls aimed at preventing outsider access "

PRINT "to material storage areas and to constraining the ability "

PRINT "of insiders to gradually divert materials."

PRINT "'!

INPUT "Input three $Y$ 6 weights "; $Y 6(1), Y 6(2), Y 6(3)$

IF ABS $(Y 6(1)+Y 6(2)+Y 6(3)-1$ !) $>$ EPS THEN

PRINT "Your weights for $Y_{-} 6$ should add up to 1.0."

PRINT "Please repeat the weight assignment."

PRINT "Touch any key to continue"

DO

LOOP UNTIL INKEY\$ <> "'"

GOTO 260

END IF

PRINT \#1, "Variable Y6 is Site Access Controls"

PRINT \#1, USING a3\$; "Y_6 values are "; Y6V(1); " "; Y6V(2); " "; Y6V(3)

PRINT \#1, USING a3\$; "Y_6 weights are "; Y6(1); " "; Y6(2); " "; Y6(3)

PRINT \#1,

\section{RETURN}

270 CLS

PRINT "Site Location"

PRINT "'!

PRINT " $Y 7=0.5$, site with none or few good escape routes"

PRINT " $Y_{-7} 7=1.5$, site with many good escape paths"

PRINT "'"

PRINT "This measures the ability of group or individual that "

PRINT "has successfully diverted material to successfully remove "

PRINT "it to a secure location and/or transfer it to the end-user. "

PRINT "'"

INPUT "Input two $Y_{-} 7$ weights "; $Y 7(1), Y 7(2)$

IF ABS $(Y 7(1)+Y 7(\overline{2})-1$ !) $>$ EPS THEN

PRINT "Your weights for $Y_{-} 7$ should add up to 1.0." 
PRINT "Please repeat the weight assignment."

PRINT "Touch any key to continue"

DO

LOOP UNTIL INKEY\$ <> "'"

GOTO 270

END IF

PRINT \#1, "Variable Y7 is Site Location (as it affects interdiction of "

PRINT \#1, " diverted materials)"

PRINT \#1, USING a2\$; "Y_7 values are "; Y7V(1); " "; Y7V(2)

PRINT \#1, USING a2\$; "Y_7 weights are "; Y7(1); " "; Y7(2)

PRINT \#1,

\section{RETURN}

280 CLS

PRINT "Emergency Response "

PRINT "'!

PRINT " $Y_{-} 8=0.5$, site with excellent emergency response resources"

PRINT " Y $8=1.0$, site with some emergency response"

PRINT " $Y_{-} 8=2.0$, site with none or poor emergency response"

PRINT "'

PRINT "This measures the ability of off-site emergency response to "

PRINT "interdict a forcible diversion in progress, given timely "

PRINT "notification. Note that measures $Y_{-} 4, Y_{-} 5$, and $Y_{-} 7$ also"

PRINT "condition the likelihood of successful interdiction of $a$ "

PRINT "diversion in progress. "

PRINT "'!

INPUT "Input three $Y \_8$ weights "; $Y 8(1), Y 8(2), Y 8(3)$

IF ABS(Y8(1) + Y8(2) + Y8(3) - 1!) > EPS THEN

PRINT "Your weights for $Y \_8$ should add up to 1.0."

PRINT "Please repeat the weight assignment."

PRINT "Touch any key to continue"

DO

LOOP UNTIL INKEY\$ <> "'!

GOTO 280

END IF

PRINT \#1, "Variable Y8 is Site Emergency Response (as it affects "

PRINT \#1, " interdiction of diverted materials)"

PRINT \#1, USING a3\$; "Y_8 values are "; Y8V(1); " "; Y8V(2); " "; Y8V(3)

PRINT \#1, USING a3\$; "Y_8 weights are "; $Y 8(1) ;$; "; $Y 8(2)$; " "; $Y 8(3)$

PRINT \#1,

\section{RETURN}

\section{CLS}

PRINT "Overall Capability of Host Country Safeguards Measures"

PRINT "'!

PRINT " Y_9 $=0.5$, Robust safeguards measures "

PRINT " $Y_{-} 9=1.0$, Safeguards with some weaknesses in design "

PRINT " or implementation "

PRINT " Y_9 $=2.0$, Weak or non-existent safeguards "

PRINT "'! 
PRINT "This measures the overall capability of safeguards measures " PRINT "to detect/alarm attempted state transfer of weapons material " PRINT "to unauthorized state- or sub-state-organizations."

PRINT "'!

INPUT "Input three $Y$ 9 weights "; $Y 9(1), Y 9(2), Y 9(3)$

IF ABS(Y9(1) + Y $9(2)+Y 9(3)-1$ !) $>$ EPS THEN

PRINT "Your weights for $Y_{-} 9$ should add up to 1.0."

PRINT "Please repeat the weight assignment."

PRINT "Touch any key to continue"

DO

LOOP UNTIL INKEY\$ $<>$

GOTO 285

END IF

PRINT \#1, "Variable Y9 is Overall Capability of Host Country Safeguards"

PRINT \#1, USING a3\$; "Y_9 values are "; Y9V(1); " "; Y9V(2); " "; Y9V(3)

PRINT \#1, USING a3\$; "Y_9 weights are "; Y9(1); " "; Y9(2); " "; $Y 9(3)$

PRINT \#1,

\section{RETURN}

290 CLS

PRINT "Socio-political stability "

PRINT "'!'

PRINT" Y_10 $=0.5$, stable government with good foreign "

PRINT " $\quad$ relations, checks and balances"

PRINT" Y Y 10 = 1.0, some instability, some foreign enemies, "

PRINT" . some checks and balances"

PRINT " Y_10 $=2.0$, significant instability, many factions, "

PRINT " - many enemies, no checks and balances"

PRINT "'!

PRINT "This attempts to measure the likelihood that all or part of "

PRINT "the relevant government organizations will formulate and "

PRINT "implement or will fail to impede a state transfer diversion "

PRINT "attempt. "

PRINT "'!

INPUT "Input three $Y \_10$ weights "; $Y 10(1), Y 10(2), Y 10(3)$

IF ABS $(Y 10(1)+Y 10(2)+Y 10(3)-1$ !) > EPS THEN

PRINT "Your weights for $Y_{-} 10$ should add up to 1.0."

PRINT "Please repeat the weight assignment."

PRINT "Touch any key to continue"

DO

LOOP UNTIL INKEY\$ <> "'"

GOTO 290

END IF

PRINT \#1, "Variable $Y 10$ is Host Country Socio-political Stability"

PRINT \#1, USING a3\$; "Y_10 values are "; Y10V(1); " "; Y10V(2); " "; Y10V(3)

PRINT \#1, USING a3\$; "Y_10 weights are "; Y10(1); " "; Y10(2); " "; Y10(3)

PRINT \#1,

\section{RETURN}


- Subroutine 3000 calculates Threat and Vulnerability Probabilities

' for the Outsider Attack scenario type

3000 GOSUB 100

GOSUB 110

GOSUB 120

GOSUB 140

GOSUB 150

GOSUB 160

GOSUB 170

GOSUB 180

GOSUB 230

GOSUB 240

GOSUB 250

GOSUB 260

GOSUB 270

GOSUB 280

DIM OATHREATV! $(3,2,2,4,3,2,3)$

DIM OATHREATP! $(3,2,2,4,3,2,3)$

DIM OAVULNV! $(3,3,3,3,2,3)$

DIM OAVULNP! $(3,3,3,3,2,3)$

FOR $11=1$ TO 3

FOR I2 $=1$ TO 2

FOR $14=1$ TO 2

FOR I5 $=1$ TO 4

FOR $16=1$ TO 3

FOR $17=1$ TO 2

FOR $18=1$ TO 3

OATHREATV! $(11,12,14,15,16,17,18)=X 1 V(11) * X 2 V(12) * X 4 V(14) * X 5 V(15) * X 6 V(16) * X 7 V(17)$ * $\mathrm{X} 8 \mathrm{~V}(18)$

OATHREATP! $(11,12,14,15,16,17,18)=X 1(11) * X 2(12) * X 4(14) * X 5(15) * X 6(16) * X 7(17) * X 8(18)$

OATHREATPROB! ((OATHREATV! $(11,12,14,15,16,17,18) * 2)+1)=$

OATHREATPROB!((OATHREATV!(11, 12, 14, 15, 16, 17, 18)*2) + 1) + OATHREATP! $(11,12,14,15,16,17,18)$

'PRINT \#1 USING C\$, "OATHREATV!("; 11; ","; 12; ","; 14; ","; 15; ","; 16; ","; 17; ","; 18; ") = ";

OATHREATV!(11, 12, 14, 15, 16, 17, 18)

'PRINT \#1 USING c\$, "OATHREATP!("; 11; ","; 12; ","; 14; ","; 15; ","; 16; ","; 17; ","; 18; ") = ";

OATHREATP!(11, 12, 14, 15, 16, 17, 18)

' PRINT \#1,

NEXT 18

NEXT 17

NEXT 16

NEXT 15

NEXT 14

NEXT 12

NEXT 11

FOR $13=1$ TO 3

FOR I4 $=1$ TO 3

FOR I5 $=1$ TO 3

FOR $16=1$ TO 3

FOR $17=1$ TO 2

FOR $18=1$ TO 3

IF $Y 5 V(15)>Y 6 V(16)$ THEN MAX1! = Y5V(15) ELSE MAX1! = Y6V(16) 
IF Y7V(17)>Y8V(18) THEN MAX2! = Y7V(17) ELSE MAX2! = Y8V(18)

OAVULNV! $(13,14,15,16,17,18)=Y 3 V(13) * Y 4 V(14) *$ MAX1! * MAX2!

OAVULNP! $(13,14,15,16,17,18)=Y 3(13) * Y 4(14) * Y 5(15) * Y 6(16) * Y 7(17) * Y 8(18)$

OAVULNPROB! $((O A V U L N V !(13,14,15,16,17,18) * 6)+1)=$ OAVULNPROB!((OAVULNV! $(13,14,15,16$, $17,18) * 6)+1)+$ OAVULNP! $(13,14,15,16,17,18)$

' PRINT \#1, USING debug2\$; "Vulnerability value factors are "; Y3V(I3); Y4V(14); MAX2!; MAX2!

' PRINT \#1, USING debug1\$; "OAVULNV!(-,-,"; 13; ","; 14; ","; I5; ","; 16; ","; 17; ","; 18; ") = "; OAVULNV!(13, $14,15,16,17,18)$

' PRINT \#1, USING debug1\$; "OAVULNP!(-,-,"; 13; ","; 14; ","; 15; ","; 16; ","; 17; ", "; 18; ") = "; OAVULNP!(13, $14,15,16,17,18$ )

'PRINT\#1,

NEXT 18

NEXT 17

NEXT 16

NEXT 15

NEXT 14

NEXT 13

' FOR I = 1 TO 101

- PRINT\#1, USING tv\$; "For Threat value = "; $(I-1) / 2 ;$ " the probability is "; OATHREATPROB!(I)

' NEXT I

PRINT \#1,

' FOR I = 1 TO 101

"PRINT \#1, USING tv\$; "For Vulnerability value = "; $(1-1) / 6$; " the probability is "; OAVULNPROB!(l)

' NEXT I

GOSUB 5000

GOSUB 5100

GOSUB 5200

\section{RETURN}

- Subroutine 5000 accumulates the Threat probabilities values into

- four Threat category probability values for the Outsider Attack

- scenario type; it also accumulates the Vulnerability probability

- values into four Vulnerability category probability values for the

' Outsider Attach scenario type.

5000 FOR I $=1$ TO 1

NEXT I

$$
\operatorname{THREAT}(1)=\text { THREAT(1) + OATHREATPROB!(1) }
$$

FOR $1=2$ TO 2

NEXT I

THREAT(2) $=$ THREAT $(2)+$ OATHREATPROB!(I)

FOR $1=3$ TO 6

THREAT(3) = THREAT $(3)+$ OATHREATPROB! $(1)$

\section{NEXT I}

FOR I = 7 TO 101 NEXT

THREAT(4) = THREAT $(4)+$ OATHREATPROB! $(1)$

PRINT \#1,

PRINT \#1, USING c\$; "For Threat category Negligible, the probability is ";THREAT(1)

PRINT \#1, USING c\$; "For Threat category Low, the probability is ";THREAT(2) 
PRINT \#1, USING c\$; "For Threat category High, the probability is ";THREAT(3)

PRINT \#1, USING c\$; "For Threat category Very High, the probability is ",THREAT(4)

PRINT \#1,

FOR $\mid=1$ TO 6

NEXT I

VULN $(1)=$ VULN $(1)+$ OAVULNPROB! $(1)$

FOR I = 7 TO 11

NEXT I

VULN(2) = VULN(2) + OAVULNPROB!(I)

FOR I $=12$ TO 21

NEXT I

VULN(3) $=$ VULN $(3)+$ OAVULNPROB!(I)

FOR $I=22$ TO 101

NEXT I

VULN(4) = VULN(4) + OAVULNPROB!(I)

PRINT \#1,

PRINT \#1, USING c\$; "For Vulnerability category Negligible, the probability is ";VULN(1)

PRINT \#1, USING C\$; "For Vulnerability category Low, the probability is "; VULN(2)

PRINT \#1, USING c\$; "For Vulnerability category High, the probability is ";VULN(3)

PRINT \#1, USING c\$; "For Vulnerability category Very High, the probability is ";VULN(4)

PRINT \#1,

RETURN

' Subroutine 5100 computes the Likelihood matrix probability values and

' accumulates them into the five Likelihood category probabilities

5100. FOR I $=1$ TO 4

FOR $\mathrm{J}=1$ TO 4

LMAT! $(I, J)=$ THREAT $(I) *$ VULN $(J)$

NEXT 1

NEXT J

LHOOD!(1) = LMAT! $(1,1)+\operatorname{LMAT!}(1,2)+\operatorname{LMAT!}(1,3)+\operatorname{LMAT}(2,1)$

LHOOD! $(2)=$ LMAT! $(1,4)+$ LMAT! $(2,2)+$ LMAT! $(3,1)+$ LMAT! $(4,1)$

LHOOD! $(3)=$ LMAT! $(2,3)+$ LMAT! $(3,2)+$ LMAT! $(4,2)$

LHOOD! (4) = LMAT! $(2,4)+\operatorname{LMAT!}(3,3)+\operatorname{LMAT!}(4,3)$

LHOOD! $(5)=$ LMAT! $(3,4)+$ LMAT! $(4,4)$

PRINT \#1,

PRINT \#1, USING c\$; "Probability for Likelihood category Negligible = "; LHOOD!(1)

PRINT \#1, USING c\$; "Probability for Likelihood category Low = "; LHOOD!(2)

PRINT \#1, USING c\$; "Probability for Likelihood category Medium = "; LHOOD!(3)

PRINT \#1, USING C\$; "Probability for Likelihood category High = "; LHOOD!(4)

PRINT \#1, USING C\$; "Probability for Likelihood category Very High = "; LHOOD!(5)

PRINT \#1,

\section{RETURN}

' Subroutine 5200 solicits input from the evaluator for the Consequence

' measure, given as weights/probabilities assigned to each of the five

- Consequence categories. This input is echoed to output. Finally,

' Risk Matrix probabilities are calculated and then accumulated into

' the five Risk category probabilities 
5200 CLS

PRINT "Enter estimate of site/facility diversion consequences."

PRINT "Enter five weights/probabilities associated with the categories:"

PRINT " Negligible = Fission products or other non-weapons material"

PRINT " (IAEA Attractiveness Level $E$ or less)

PRINT "m!

PRINT " Low = Small amounts of difficult to convert materials"

PRINT " $\quad$ (IAEA Attractiveness Level D, Category IV)"

PRINT "'!

PRINT "

PRINT "

Medium = Small amounts of easily convertible or large " amounts of difficult to convert materials"

PRINT "

PRINT "

PRINT "'

PRINT "

PRINT "

PRINT "

PRINT "

PRINT "'"

PRINT "

PRINT "

PRINT "'!

(IAEA Attractiveness Level D, Categories I,II, or III"

and Attractiveness Levels B and C, Category IV)"

High = Large amounts of fissile material easily convertible" to weapons"

(IAEA Attractiveness Level B and C, "

Categories I,II, or III)"

INPUT "Input the five weights "; CONSEQ(1), CONSEQ(2), CONSEQ(3), CONSEQ(4), CONSEQ(5)

IF ABS(CONSEQ(1) + CONSEQ(2) + CONSEQ(3) + CONSEQ(4) + CONSEQ(5) - 1!) > EPS THEN

PRINT "Your weights for CONSEQ(I) should add up to 1.0."

PRINT "Please repeat the weight assignment."

PRINT "'"

PRINT "Touch any key to continue"

DO

LOOP UNTIL INKEY\$ <> "'"

GOTO 290

END IF

PRINT \#1,

PRINT \#1, USING c\$; "Probability for Consequence category Negligible = "; CONSEQ(1)

PRINT \#1, USING c\$; "Probability for Consequence category Low = "; CONSEQ(2)

PRINT \#1, USING c\$; "Probability for Consequence category Medium = "; CONSEQ(3)

PRINT \#1, USING c\$; "Probability for Consequence category High = "; CONSEQ(4)

PRINT \#1, USING c\$; "Probability for Consequence category Very High = "; CONSEQ(5)

PRINT \#1,

FOR I = 1 TO 5

FOR $\mathrm{J}=1$ TO 5

$\operatorname{RISK}(I, J)=\operatorname{LHOOD!}(I){ }^{*} \operatorname{CONSEQ}(J)$

NEXT I

NEXT $J$

$\operatorname{RISKCAT}(1)=\operatorname{RISK}(1,1)+\operatorname{RISK}(1,2)+\operatorname{RISK}(1,3)+\operatorname{RISK}(2,1)+\operatorname{RISK}(3,1)+\operatorname{RISK}(4,1)+\operatorname{RISK}(5,1)$

$\operatorname{RISKCAT}(2)=\operatorname{RISK}(2,3)+\operatorname{RISK}(3,2)+\operatorname{RISK}(4,2)+\operatorname{RISK}(5,2)+\operatorname{RISK}(2,2)+\operatorname{RISK}(1,4)$

$\operatorname{RISKCAT}(3)=\operatorname{RISK}(2,4)+\operatorname{RISK}(3,3)+\operatorname{RISK}(1,5)+\operatorname{RISK}(5,3)+\operatorname{RISK}(4,3)$

$\operatorname{RISKCAT}(4)=\operatorname{RISK}(2,5)+\operatorname{RISK}(3,5)+\operatorname{RISK}(3,4)+\operatorname{RISK}(4,4)+\operatorname{RISK}(5,4)$ 
$\operatorname{RISKCAT}(5)=\operatorname{RISK}(4,5)+\operatorname{RISK}(5,5)$

PRINT \#1,

PRINT \#1, USING c\$; "Probability for Risk Category Negligible = "; RISKCAT(1)

PRINT \#1, USING c\$; "Probability for Risk Category Low = "; RISKCAT(2)

PRINT \#1, USING c\$; "Probability for Risk Category Medium = "; RISKCAT(3)

PRINT \#1, USING c\$; "Probability for Risk Category High = "; RISKCAT(4)

PRINT \#1, USING C\$; "Probability for Risk Category Very High = "; RISKCAT(5)

PRINT \#1,

\section{RETURN}

END 


\section{Source code for the Outsider Theft Scenario}

DEFSNG A-H, O-Z

DEFINT I-N

OPTION BASE 1

$\mathrm{EPS}=.001$

$\operatorname{DIM} \times 1(3)$

DIM X2(2)

$\operatorname{DIM} \times 3(3)$

DIM X4(2)

DIM X5(4)

DIM X6(3)

DIM X7(2)

DIM X8(3)

DIM Y1(3)

DIM Y2(3)

DIM Y3(3)

DIM Y4(3)

DIM Y5(3)

DIM Y6(3)

DIM Y7(2)

DIM Y8(3)
' Blood feud -- WEIGHTS

' Political/ideological motivations - WEIGHTS

' Religious motivations -- WEIGHTS

- Perception of target vulnerability - WEIGHTS

' Availability of resources - WEIGHTS

- Training and leadership - WEIGHTS

' Knowledge and intelligence - WEIGHTS

' Site/facility socio-economic conditions - WEIGHTS

' Material surveillance -- WEIGHTS

- Transfer controls -- WEIGHTS

- Protective forces -- WEIGHTS

- Intrusion detection -- WEIGHTS

' Barriers/delays - WEIGHTS

' Access controls - WEIGHTS

' Site location -- WEIGHTS

' Emergency response -- WEIGHTS 


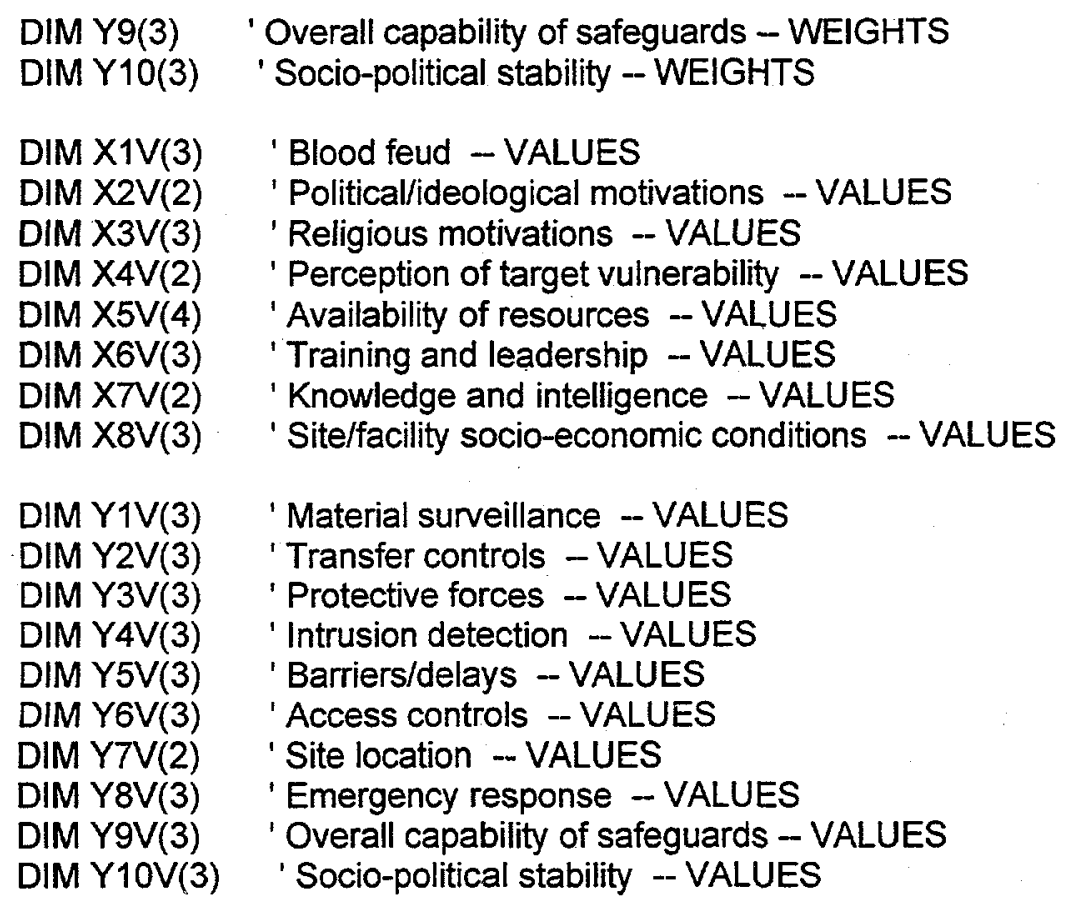


DATA $0.5,1.5$

FOR I = 1 TO 3: READ Y8V(I): NEXT I

DATA $0.5,1.0,2.0$

FOR I = 1 TO 3: READ Y9V(I): NEXT I

DATA $0.5,1.0,2.0$

FOR I = 1 TO 3: READ Y10V(I): NEXT I

DATA $0.5,1.0,2.0$

DIM OTTHREATPROB!(101)

DIM OTVULNPROB!(101)

DIM THREAT(4) 'Threat category probabilities

DIM VULN(4) 'Vulnerability category probabilities

DIM LHOOD!(5) 'Likelihood category probabilities

DIM LMAT! $(4,4)$ 'Likelihood matrix probabilities

DIM CONSEQ(5) 'Consequence category probabilities

DIM RISK $(5,5)$ 'Risk matrix probabilities

DIM RISKCAT(5) 'Risk category probabilities

DIM scentype\$(4) ' Scenario type names

FOR I = 1 TO 4: READ scentype\$(I): NEXT I

DATA "Insider theft", "Outsider theft", "Outsider attack", "State transfer"

INPUT "Enter name of output file "; outfile\$

OPEN outfile\$ FOR OUTPUT AS \#1

' Define printout formats

a2\$ = "\&\#.\#\# \&\#.\#"

a3\$ $=$ "\&\#.\#\# \&\#.\#\# \&\#.\#"

a4\$ = "\&\#.\#\# \&\#.\#\# \& \#.\#\# \&\#.\#\#"

tv\$ = "\& \#\#\#\# \&\#.\#"

$C \$=" \& \#$

IC\$ = "\&\#\&\#.\#"

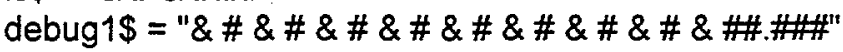

debug2\$ = "\&\#.\#\#.\#\#\#.\#\#.\#\#.\#\#"

INPUT "Enter site/facility name "; sitename\$

PRINT \#1, "Evaluation of site/facility: "; sitename\$

PRINT \#1, "For scenario type: $\quad$ "; scentype\$(2)

PRINT \#1, "Output file name: $\quad$ "; outfile\$

PRINT \#1, "Program and Version: Version 4 of OT_EVAL"

PRINT \#1,

GOSUB 2000

STOP

' Subroutines 110 through 290 solicit evaluator input for Threat and

' Vulnerability sub-measures. Subroutine 100 explains that the Threat

' and Vulnerability sub-measures are all treated as random variables, 
' requiring that probabilities (or weights) summing to 1 must be assigned

' to each of the numerical values taken on by that sub-measure.

100 CLS

PRINT "The Threat and Vulnerability sub-measures used in this scheme"

PRINT "for estimating the risk of diversion of nuclear materials are"

PRINT "all treated as random variables, requiring that probabilities"

PRINT "(or weights) summing to 1 must be assigned to each of the"

PRINT "numerical values taken on by that sub-measure. The input routines"

PRINT "will enforce this requirement."

PRINT 'm!

PRINT "In addition, the assignment of probabilities to the sub-measure"

PRINT "can be used to represent the evaluators uncertainty about the"

PRINT "correct value of the sub-measure. For instance, in the absence of"

PRINT "any information about the true value of the sub-measure"

PRINT "(i.e., complete uncertainty), the evaluator should assign equal"

PRINT "weights to all of the sub-measure values."

PRINT 'm!

PRINT "Touch any key to continue"

DO

LOOP UNTIL INKEY\$ <> "'

RETURN

110 CLS

PRINT "Existence of a Long Standing Conflict "

PRINT "'"

PRINT " X_1 $=0.5$, no underlying conflict"

PRINT " $\quad X \_1=1.0$, recently developed conflict (decades-long duration)"

PRINT " $\quad X_{-} 1=2.0$, long standing conflict (centuries-long duration)"

PRINT "'!

PRINT "*Long Standing Conflict" is shorthand for aspects of intensity "

PRINT "and long duration in the motivation of the threat groups. It can "

PRINT "derive from extreme religious prejudice, language/cultural "

PRINT "differences, and/or from a long history of oppression,"

PRINT "suppression, or warfare between two groups of people. Examples "

PRINT "include Catholic Irish vs. Irish Protestants and the British "

PRINT "or the Basques vs. the Spanish or Islamic fundamentals vs. "

PRINT "secular Moslem governments. "

PRINT "'!

INPUT "Input three X_1 weights "; X1(1), X1(2), X1(3)

IF ABS $(X 1(1)+X 1(2)+X 1(3)-1 !)>$ EPS THEN

PRINT "Your weights for $X \_1$ should add up to 1.0."

PRINT "Please repeat the weight assignment."

PRINT "Touch any key to continue"

DO

LOOP UNTIL INKEY\$ <> "'"

GOTO 110

END IF

PRINT \#1, "Variable $X 1$ is Existence of a Long Standing Conflict"

PRINT \#1, USING a3\$; "X_1 values are "; X1V(1); " "; X1V(2); " "; X1V(3)

PRINT \#1, USING a3\$; "X_1 weights are "; X1(1); " "; X1(2); " "; $X 1(3)$ 
PRINT \#1,

RETURN

120 CLS

PRINT "Political//deological Motivations "

PRINT "'!

PRINT " X_2 $=0.5$, no political/ideological basis "

PRINT " X_2 $=1.0$, political/ideological motivations"

PRINT "'"

PRINT "Examples would be support for terrorism as a component "

PRINT "of the cold war struggle between Communism and the West."

PRINT "'

INPUT "Input two X_2 weights "; $X 2(1), X 2(2)$

IF ABS $(X 2(1)+X 2(\overline{2})-1$ !) $>$ EPS THEN

PRINT "Your weights for $X \_2$ should add up to 1.0."

PRINT "Please repeat the weight assignment."

PRINT "Touch any key to continue"

DO

LOOP UNTIL INKEY\$ $<>" '$

GOTO 120

END IF

PRINT \#1, "Variable $X 2$ is Political/ldeological Motivations"

PRINT \#1, USING a2\$; "X_2 values are "; X2V(1); " "; X2V(2)

PRINT \#1, USING a2\$; "X_2 weights are "; X2(1); " "; X2(2)

PRINT \#1,

RETURN

130 CLS

PRINT "Religious Motivations "

PRINT" "

PRINT " X_3 $=0.5$, no religious animosity"

PRINT" $\quad X \_3=1.0$, some religious animosity"

PRINT " $\quad X \_3=2.0$, strong religious animosity"

PRINT "'"

PRINT "The intent here is to capture aspects of motivation distinct "

PRINT "from possible religious components of the "; blood; feud; " measure "

PRINT "above."

PRINT "'!

INPUT "Input three X_3 weights "; X3(1), X3(2), X3(3)

IF ABS $(X 3(1)+X 3(2)+X 3(3)-1$ !) $>$ EPS THEN

PRINT "Your weights for $X \_3$ should add up to 1.0."

PRINT "Please repeat the weight assignment."

PRINT "Touch any key to continue"

DO

LOOP UNTIL INKEY\$ <> "'"

GOTO 130

END IF

PRINT \#1, "Variable X3 is Religious Motivations"

PRINT \#1, USING a3\$; "X_3 values are "; X3V(1); " "; X3V(2); " "; X3V(3) 
PRINT \#1, USING a3\$; "X_3 weights are "; X3(1); " "; X3(2); " "; X3(3)

PRINT \#1,

\section{RETURN}

140 CLS

PRINT "Perception of target vulnerability "

PRINT "'!

PRINT" $\quad X \_4=0.5$, facility not perceived as vulnerable "

PRINT " X_4 = 1.5, facility perceived as vulnerable"

PRINT "'"

PRINT "The reasoning here is that, even for a threat group predisposed "

PRINT "to action, if a particular facility is perceived as not vulnerable, "

PRINT "they will direct their attention elsewhere."

PRINT '"!

INPUT "Input two $X \_4$ weights "; $X 4(1), X 4(2)$

IF ABS $(X 4(1)+X 4(\overline{2})-1$ !) $>$ EPS THEN

PRINT "Your weights for $X \_4$ should add up to 1.0."

PRINT "Please repeat the weight assignment.".

PRINT "Touch any key to continue"

DO

LOOP UNTIL INKEY\$ <> "'"

GOTO 140

END IF

PRINT \#1, "Variable X4 is Perception of Target Vulnerability"

PRINT \#1, USING a2\$; "X_4 values are "; X4V(1); " "; X4V(2)

PRINT \#1, USING a2\$; "X_4 weights are "; X4(1); " "; X4(2)

PRINT \#1,

\section{RETURN}

150 CLS

PRINT "Availability of resources "

PRINT "'"

PRINT " X_5 $=0.0$, no resources available"

PRINT " X_5 $=0.5$, modest, locally collected resources"

PRINT " X_5 =1.0, intermediate, state-supported"

PRINT " X_5 $=2.0$, large, state-supported"

PRINT "'!

PRINT "This is a measure of the resource base of the threat group."

PRINT '"'

INPUT "Input four X_5 weights "; X5(1), X5(2), X5(3), X5(4)

IF ABS $(X 5(1)+X 5(\overline{2})+X 5(3)+X 5(4)-1$ !) $>$ EPS THEN

PRINT "Your weights for $X \_5$ should add up to 1.0."

PRINT "Please repeat the weight assignment."

PRINT "Touch any key to continue"

DO

LOOP UNTIL INKEYS <> "'"

END IF

GOTO 150

PRINT \#1, "Variable X5 is Availability of Threat Group Resources"

PRINT \#1, USING a4\$; "X_5 values are "; X5V(1); "; X5V(2); " "; X5V(3); " "X5V(4) 
PRINT \#1, USING a4\$; "X_5 weights are "; X5(1); " "; X5(2); " "; X5(3); " "; X5(4)

PRINT \#1,

\section{RETURN}

$160 \mathrm{CLS}$

PRINT "Training and leadership "

PRINT "'!

PRINT " X_6 $=0.5$, poor training and leadership"

PRINT " X_6 $=1.0$, intermediate training and leadership"

PRINT " X_6 $=2.0$, well-trained and led"

PRINT '"'

PRINT "This is a measure of the quality of training and leadership "

PRINT "of the threat organization."

PRINT "'"

INPUT "Input three X_6 weights "; X6(1), X6(2), X6(3)

IF ABS(X6(1)+X6(2) + X6(3) - 1!) > EPS THEN

PRINT "Your weights for $X \_6$ should add up to 1.0."

PRINT "Please repeat the weight assignment."

PRINT "Touch any key to continue"

DO

LOOP UNTIL INKEY\$ <> "'!

GOTO 160

END IF

PRINT \#1, "Variable X6 is Threat Group Training and Leadership"

PRINT \#1, USING a3\$; "X_6 values are "; X6V(1); " "; X6V(2); " "; X6V(3)

PRINT\#1, USING a3\$; "X_6 weights are "; X6(1); " "; X6(2); " "; X6(3)

PRINT \#1,

\section{RETURN}

170 CLS

PRINT "Knowledge and intelligence "

PRINT "'"

PRINT " $\quad X \_7=0.5$, poor intelligence capabilities"

PRINT " $\quad X_{-}^{-7}=2.0$, good intelligence capabilities"

PRINT "'"

PRINT "This is a measure of the access of the threat organization "

PRINT "to information about the potential target and their ability "

PRINT "to use that information."

PRINT "'"

INPUT "Input two $X \_7$ weights "; $X 7(1), X 7(2)$

IF ABS $(X 7(1)+X 7(\overline{2})-1$ !) $>$ EPS THEN

PRINT "Your weights for $X \_7$ should add up to 1.0."

PRINT "Please repeat the weight assignment."

PRINT "Touch any key to continue"

DO

LOOP UNTIL INKEY\$ <> "'"

END IF

GOTO 170

PRINT \#1, "Variable $X 7$ is Threat Group Knowledge and Intelligence Capability"

PRINT \#1, USING a2\$; "X_7 values are "; X7V(1); " "; X7V(2) 
PRINT \#1, USING a2\$; "X_7 weights are "; $X 7(1) ;$ " "; $X 7(2)$

PRINT \#1,

\section{RETURN}

180 CLS

PRINT "Site/facility socio-economic conditions "

PRINT "'r

PRINT " X_8 $=0.75$, good conditions"

PRINT " X_8 $=1.0$, not-so-good"

PRINT " X_8 $=1.5$, extremely difficult conditions"

PRINT "'"

PRINT "This attempts to measure the likelihood that the threat "

PRINT "organization can subvert/coopt insiders to support its "

PRINT "diversion attempt or that insiders will themselves attempt diversion."

PRINT '"'

INPUT "Input three $X \_8$ weights "; $X 8(1), X 8(2), X 8(3)$

IF ABS(X8(1) + X8(2) + X8(3) - 1!) > EPS THEN

PRINT "Your weights for $X \_8$ should add up to 1.0."

PRINT "Please repeat the weight assignment."

PRINT "Touch any key to continue"

DO

LOOP UNTIL INKEYS <> "'

GOTO 180

END IF

PRINT \#1, "Variable X8 is Site/Facility Socio-Economic Conditions"

PRINT \#1, USING a3\$; "X_8 values are "; X8V(1); " "; X8V(2); " "; X8V(3)

PRINT \#1, USING a3\$; "X_8 weights are "; $X 8(1) ; "$ "; $X 8(2)$; " "; $X 8(3)$

PRINT \#1,

\section{RETURN}

210 CLS

PRINT "Material Surveillance "

PRINT '"'

PRINT " $\quad Y \_1=0.5$, excellent system of material surveillance"

PRINT " $Y_{-1}^{-1}=1.0$, good system of material surveillance"

PRINT " $Y_{-} 1=2.0$, poor system of material surveillance"

PRINT "'"

PRINT "This measures the material accounting and surveillance "

PRINT "infrastructure that enables facility management to detect "

PRINT "attempted or successful diversion."

PRINT "'!

INPUT "Input three $Y_{-} 1$ weights "; $Y 1(1), Y 1(2), Y 1(3)$

IF ABS(Y1(1) + Y1(2) + Y1(3) - 1!) > EPS THEN

PRINT "Your weights for $Y_{-}$1 should add up to 1.0."

PRINT "Please repeat the weight assignment."

PRINT "Touch any key to continue"

DO

LOOP UNTIL INKEY\$ <> "'"

GOTO 210 


\section{END IF}

PRINT \#1, "Variable $Y 1$ is Site Material Surveillance"

PRINT \#1, USING a3\$; "Y_1 values are "; Y1V(1); " "; Y1V(2); " "; Y1V(3)

PRINT \#1, USING a3\$; "Y_1 weights are "; Y1(1); " "; Y1(2); " "; Y1(3)

PRINT \#1,

\section{RETURN}

220 CLS

PRINT "Transfer Controls"

PRINT '"'

PRINT " Y Y $2=0.5$, excellent system of transfer control"

PRINT " $Y_{2} 2=1.0$, good system of transfer control"

PRINT " Y $Y_{-2}=2.0$, poor system of transfer control"

PRINT "'

PRINT "This measures the security of material in transit from one "

PRINT "storage/utilization location to another."

PRINT '"'

INPUT "Input three $Y \_2$ weights "; Y2(1), Y2(2), Y2(3)

IF ABS(Y2(1) + Y2(2) + Y2(3) - 1!) > EPS THEN

PRINT "Your weights for $Y_{2} 2$ should add up to 1.0."

PRINT "Please repeat the weight assignment."

PRINT "Touch any key to continue"

DO

LOOP UNTIL INKEY\$ $<>$ "'

GOTO 220

END IF

PRINT \#1, "Variable Y2 is Site Transfer Controls"

PRINT \#1, USING a3\$; "Y_2 values are "; Y2V(1); " "; Y2V(2); " "; Y2V(3)

PRINT \#1, USING a3\$; "Y_2 weights are "; Y2(1); " "; Y2(2); " "; Y2(3)

PRINT \#1,

\section{RETURN}

230 CLS

PRINT "Protective Forces"

PRINT "'!

PRINT " Y_3 $=0.5$, excellent protective forces"

PRINT " Y_ $3=1.0$, good protective forces"

PRINT " Y_3 $=2.0$, poor protective forces"

PRINT "'"

PRINT "This is a measure of the ability of the facility guard forces "

PRINT "to prevent or delay a diversion."

PRINT "m

INPUT "Input three $Y \_3$ weights "; $Y 3(1), Y 3(2), Y 3(3)$

IF ABS $(Y 3(1)+Y 3(2)+Y 3(3)-1$ !) $>$ EPS THEN

PRINT "Your weights for $Y_{3} 3$ should add up to 1.0."

PRINT "Please repeat the weight assignment."

PRINT "Touch any key to continue"

DO

LOOP UNTIL INKEY\$ $<>$ "'

GOTO 230 


\section{END IF}

PRINT \#1, "Variable Y3 is Site Protective Forces"

PRINT \#1, USING a3\$; "Y_3 values are "; Y3V(1); " "; Y3V(2); " "; Y3V(3)

PRINT \#1, USING a3\$; "Y_3 weights are "; Y3(1); " "; Y3(2); " "; Y3(3)

PRINT \#1,

\section{RETURN}

240 CLS

PRINT "Intrusion Detection "

PRINT 'm!

PRINT " Y $4=0.5$, excellent intrusion detection"

PRINT " Y_4 $=1.0$, good intrusion detection"

PRINT " Y_4 $=2.0$, poor intrusion detection"

PRINT '"'

PRINT "This measures infrastructure provisions for the detection of "

PRINT "outsider attack or theft attempts."

PRINT "'"

INPUT "Input three $Y \_4$ weights "; $Y 4(1), Y 4(2), Y 4(3)$

IF ABS $(Y 4(1)+Y 4(2)+Y 4(3)-1$ !) $>$ EPS THEN

PRINT "Your weights for $Y_{-} 4$ should add up to 1.0."

PRINT "Please repeat the weight assignment."

PRINT "Touch any key to continue"

DO

LOOP UNTIL INKEY\$ <> "'!

END IF

GOTO 240

PRINT \#1, "Variable Y4 is Site Intrusion Detection"

PRINT \#1, USING a3\$; "Y_4 values are "; Y4V(1); " "; Y4V(2); " "; Y4V(3)

PRINT \#1, USING a3\$; "Y_4 weights are "; Y4(1); " "; Y4(2); " "; Y4(3)

PRINT \#1,

\section{RETURN}

250 CLS

PRINT "Barriers/Delays "

PRINT "'"

PRINT " Y_5 $=0.5$, excellent barriers/delays"

PRINT " Y $5=1.0$, good barriers/delays"

PRINT " Y_5 $=2.0$, poor barriers/delays"

PRINT "'"

PRINT "This measures facility design and operation features that "

PRINT "tend to delay a diversion attempt long enough for response "

PRINT "of off-site protection forces."

PRINT "'!

INPUT "Input three $Y \_5$ weights "; $Y 5(1), Y 5(2), Y 5(3)$

IF ABS(Y5(1) + Y5(2) + Y5(3) - 1!) > EPS THEN

PRINT "Your weights for $Y \_5$ should add up to 1.0."

PRINT "Please repeat the weight assignment."

PRINT "Touch any key to continue"

DO

LOOP UNTIL INKEY\$ <> '"' 


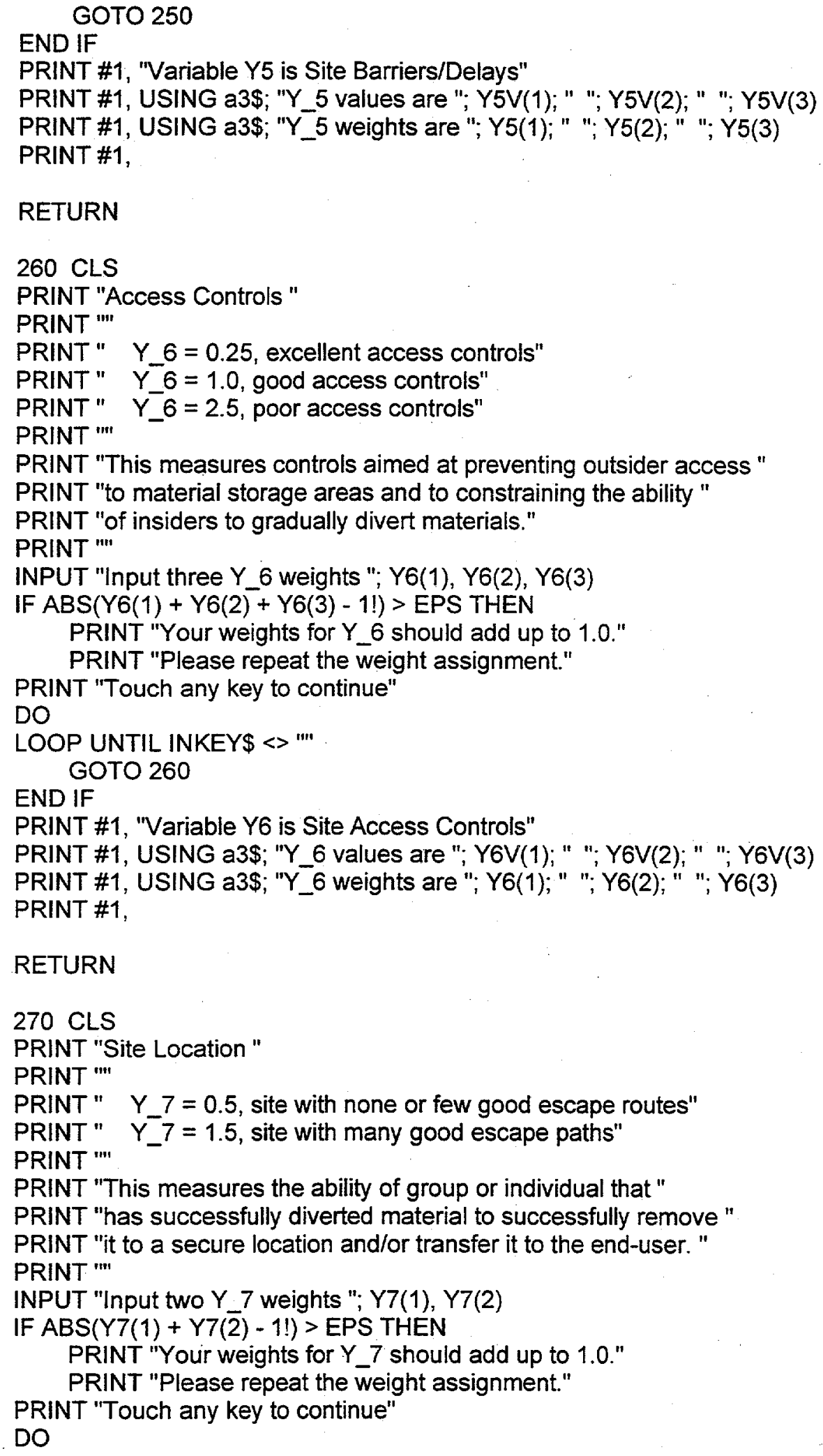




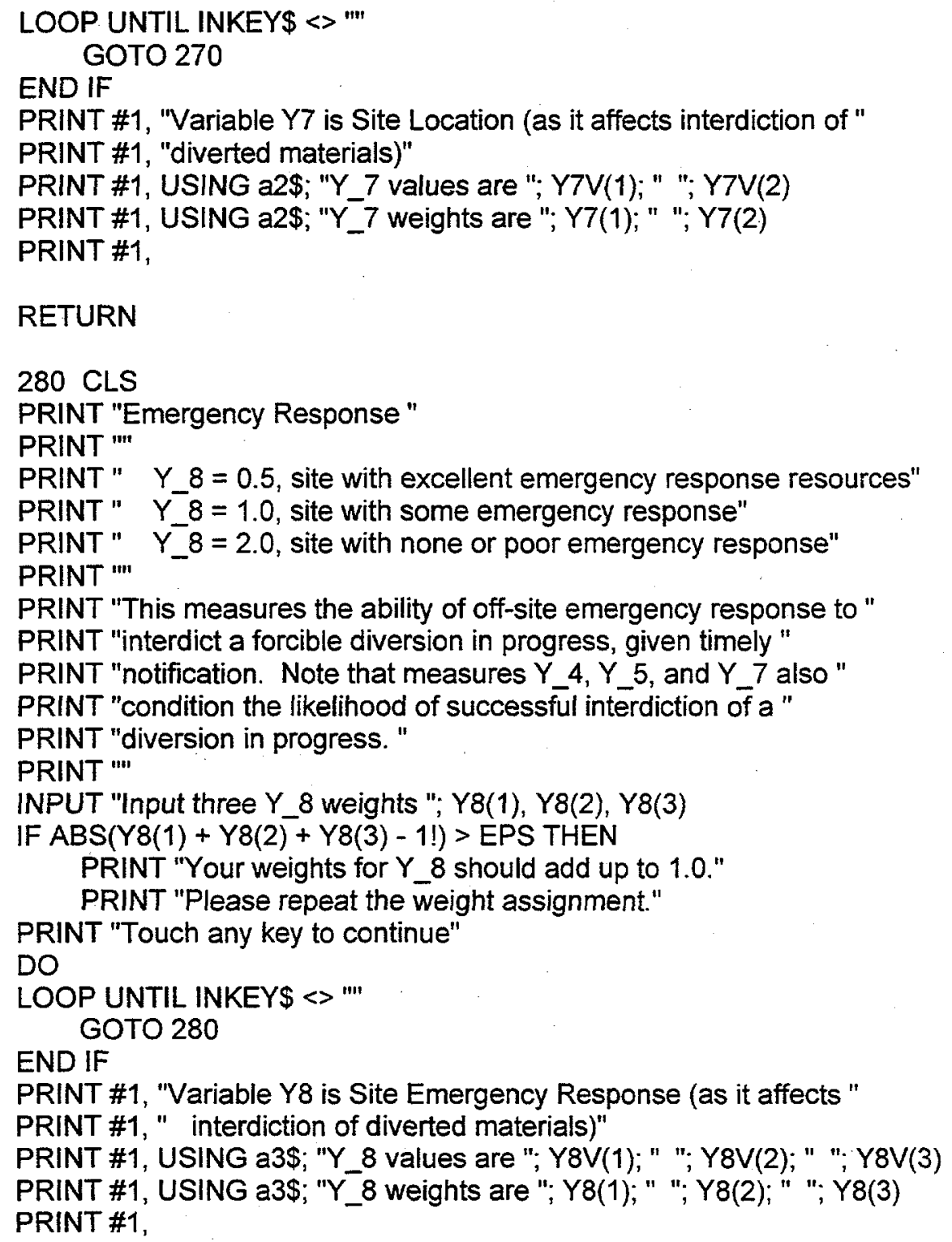

PRINT "This measures the overall capability of safeguards measures " PRINT "to detect/alarm attempted state transfer of weapons material " PRINT "to unauthorized state- or sub-state-organizations." 
PRINT "'

INPUT "Input three $Y$ 9 weights "; $Y 9(1), Y 9(2), Y 9(3)$

IF ABS $(Y 9(1)+Y 9(2)+Y 9(3)-1$ !) > EPS THEN

PRINT "Your weights for $Y \_9$ should add up to 1.0."

PRINT "Please repeat the weight assignment."

PRINT "Touch any key to continue"

DO

LOOP UNTIL INKEY\$ <> "'!

GOTO 285

END IF

PRINT \#1, "Variable $Y 9$ is Overall Capability of Host Country Safeguards"

PRINT \#1, USING a3\$; "Y_9 values are "; Y9V(1); " "; Y9V(2); " "; Y9V(3)

PRINT \#1, USING a3\$; "Y_9 weights are "; Y9(1); " "; Y9(2); " "; Y9(3)

PRINT \#1,

RETURN

290 CLS

PRINT "Socio-political stability "

PRINT "'"

PRINT" Y_10 $=0.5$, stable government with good foreign "

PRINT " $\quad$ relations, checks and balances"

PRINT " Y_10 $=1.0$, some instability, some foreign enemies, "

PRINT " - some checks and balances"

PRINT" $Y_{-} 10=2.0$, significant instability, many factions, "

PRINT " - many enemies, no checks and balances"

PRINT "'!

PRINT "This attempts to measure the likelihood that all or part of "

PRINT "the relevant government organizations will formulate and "

PRINT "implement or will fail to impede a state transfer diversion "

PRINT "attempt. "

PRINT "'!

INPUT "Input three $Y_{-} 10$ weights "; $Y 10(1), Y 10(2), Y 10(3)$

IF ABS $(Y 10(1)+Y 10 \overline{(2)}+Y 10(3)-1$ !) > EPS THEN

PRINT "Your weights for $Y_{-} 10$ should add up to 1.0."

PRINT "Please repeat the weight assignment."

PRINT "Touch any key to continue"

$\mathrm{DO}$

LOOP UNTIL INKEY\$ <> "'"

GOTO 290

END IF

PRINT \#1, "Variable Y10 is Host Country Socio-political Stability"

PRINT \#1, USING a3\$; "Y_10 values are "; Y10V(1); " "; Y10V(2); " "; Y10V(3)

PRINT\#1, USING a3\$; "Y_10 weights are "; Y10(1);" "; Y10(2);" "; Y10(3)

PRINT \#1,

RETURN

Subroutine 2000 calculates Threat and Vulnerability Probabilities

' for the Outsider Theft scenario type 
2000 GOSUB 100

GOSUB 140

GOSUB 150

GOSUB 170

GOSUB 180

GOSUB 220

GOSUB 230

GOSUB 240

GOSUB 250

GOSUB 260

GOSUB 270

GOSUB 280

DIM OTTHREATV! $(2,4,2,3)$

DIM OTTHREATP! $(2,4,2,3)$

' DIM OTVULNV! $(3,3,3,3,3,2,3)$

' DIM OTVULNP! $(3,3,3,3,3,2,3)$

FOR I $=1$ TO 2

FOR $\mathrm{J}=1$ TO 4

FOR $K=1$ TO 2

FOR L $=1$ TO 3

OTTHREATV! $(\mathrm{I}, \mathrm{J}, \mathrm{K}, \mathrm{L})=\mathrm{X} 4 \mathrm{~V}(\mathrm{I}) * \mathrm{X} 5 \mathrm{~V}(\mathrm{~J}) * \mathrm{X7V}(\mathrm{K}) * \mathrm{X} 8 \mathrm{~V}(\mathrm{~L})$

OTTHREATP! $(I, J, K, L)=X 4(I) * X 5(J) * X 7(K) * X 8(L)$

OTTHREATPROB!((OTTHREATV! $(I, J, K, L) * 5)+1)=$ OTTHREATPROB!((OTTHREATV!(I, J, K, L)

* 5) + 1) + OTTHREATP! (I, J, K, L)

' PRINT \#1, "OTTHREATV!(-,-,-,"; l; ","; J; ",-,"; L; ",",; L; ") = "; OTTHREATV!(I, J, K, L)

' PRINT \#1, "OTTHREATP!(-,-,-,"; I; ","; J; ",-,"; L; ","; L; ") = "; OTTHREATP!(I, J, K, L)

'PRINT \#1,

NEXT L

NEXTK

NEXT J

NEXT I

FOR $12=1$ TO 3

FOR I $3=1$ TO 3

FOR $14=1$ TO 3

FOR $15=1$ TO 3

FOR $16=1$ TO 3

FOR $17=1$ TO 2

FOR $18=1$ TO 3

IF Y5V(I5)>Y6V(16) THEN MAX1! = Y5V(15) ELSE MAX1! = Y6V(16)

IF $Y 7 V(17)>Y 8 V(18)$ THEN MAX2! = Y7V(17) ELSE MAX2! = Y8V(I8)

OTVULNV! $=Y 2 V(12) * Y 3 V(13) * Y 4 V(14) * M A X 1 ! * M A X 2 !$

OTVULNP! $=Y 2(12) * Y 3(13) * Y 4(14) * Y 5(15) * Y 6(16) * Y 7(17) * Y 8(18)$

OTVULNPROB!((OTVULNV! * 2.5) + 1) = OTVULNPROB! ((OTVULNV! * 2.5) + 1) + OTVULNP!

- OTVULNV! $(12,13,14,15,16,17,18)=\mathrm{Y} 2 \mathrm{~V}(12){ }^{*} \mathrm{Y} 3 \mathrm{~V}(13){ }^{*} \mathrm{Y} 4 \mathrm{~V}(14)$ * MAX1! * MAX2!

' OTVULNP! $(12,13,14,15,16,17,18)=Y 2(12) * Y 3(13) * Y 4(14) * Y 5(15) * Y 6(16) * Y 7(17)$ * $Y 8(18)$

' OTVULNPROB!((OTVULNV!(12, 13,14, 15, 16,17,18)*2.5) + 1) = OTVULNPROB!((OTVULNV!(12, 13, $14,15,16,17,18) * 2.5)+1)+$ OTVULNP! $(12,13,14,15,16,17,18)$

'PRINT \#1, USING debug2\$; "Vulnerability value factors are "; Y2V(I2); Y3V(I3); Y4V(14); MAX1!; MAX2! ' PRINT \#1, USING debug1\$; "OTVULNV!(-,"; 11; ","; I2; ","; I3; ",", 14; ","; 15; ","; 16; ","; 17; ","; 18; " ) = "; OTVULNV!

'PRINT \#1, USING debug1\$; "OTVULNP!(-,"; 11; ","; 12; ","; 13; ","; 14; ","; 15; ","; 16; ","; 17; ","; 18; ",-) = "; OTVULNP! 


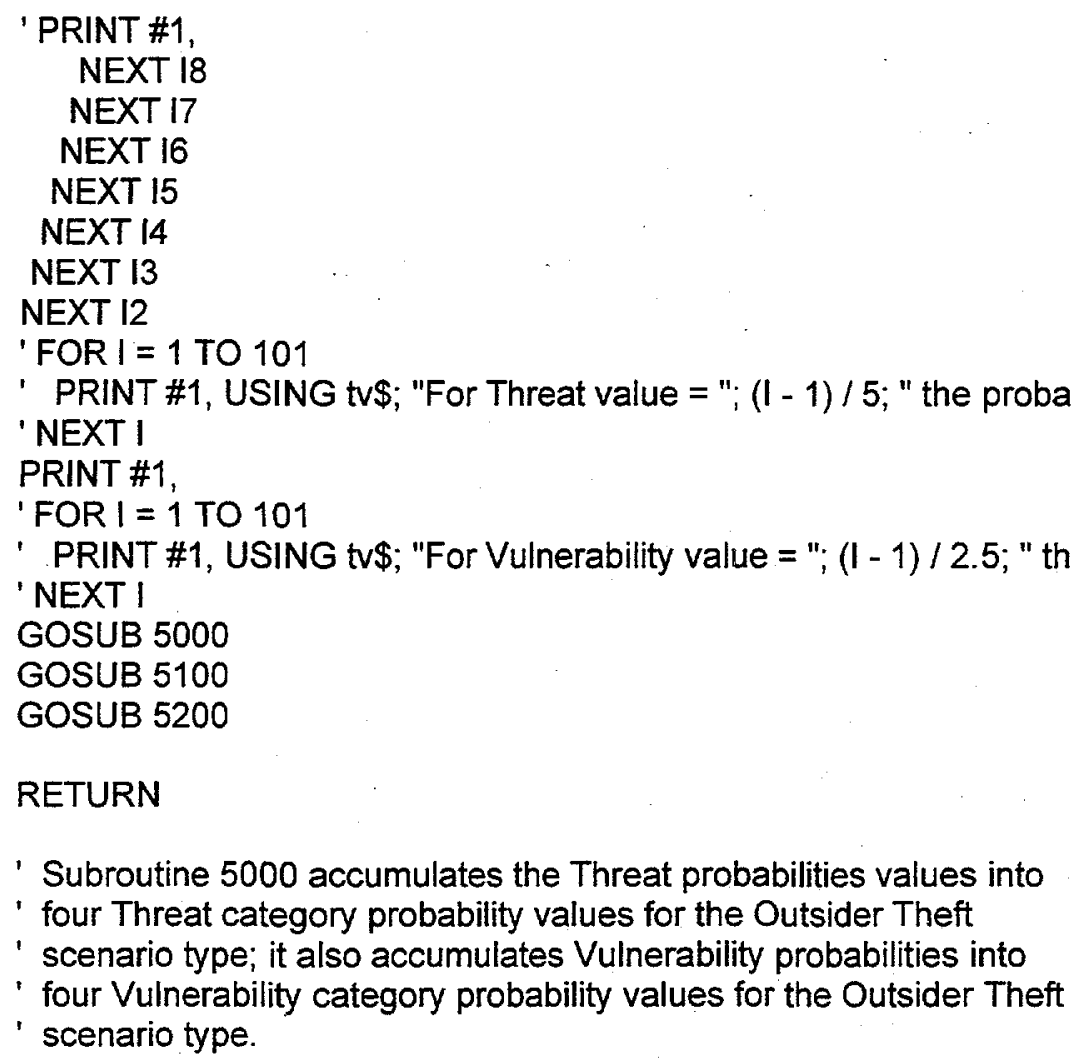
' NEXT 1

\section{RETURN}

' Subroutine 5000 accumulates the Threat probabilities values into

' four Threat category probability values for the Outsider Theft

' scenario type; it also accumulates Vulnerability probabilities into

' four Vulnerability category probability values for the Outsider Theft

' scenario type.

' PRINT \#1, USING tv\$; "For Vulnerability value = "; (I - 1) / 2.5; " the probability is "; OTVULNPROB!(I)

5000 FORI $=1$ TO 2

$$
\begin{aligned}
& \text { THREAT }(1)=\text { THREAT }(1)+\text { OTTHREATPROB! }(1) \\
& \text { NEXT I }
\end{aligned}
$$$$
\text { FOR I }=3 \text { TO } 5
$$

$$
\begin{aligned}
& \text { THREAT }(2)=\text { THREAT }(2)+\text { OTTHREATPROB! }(1) \\
& \text { NEXT I }
\end{aligned}
$$$$
\text { FOR I }=6 \text { TO } 12
$$

$$
\begin{aligned}
& \text { THREAT }(3)=\text { THREAT(3) + OTTHREATPROB! }(1) \\
& \text { NEXT I }
\end{aligned}
$$

$$
\text { FOR I = } 13 \text { TO } 101
$$

print \#1,

$$
\text { THREAT(4) = THREAT(4) + OTTHREATPROB!(I) }
$$

PRINT \#1, USING c\$; "For Threat category Negligible, the probability is ";THREAT(1)

PRINT \#1, USING c\$; "For Threat category Low, the probability is ";THREAT(2)

PRINT \#1, USING c\$; "For Threat category High, the probability is ";THREAT(3)

PRINT \#1, USING c\$; "For Threat category Very High, the probability is ";THREAT(4) print \#1,

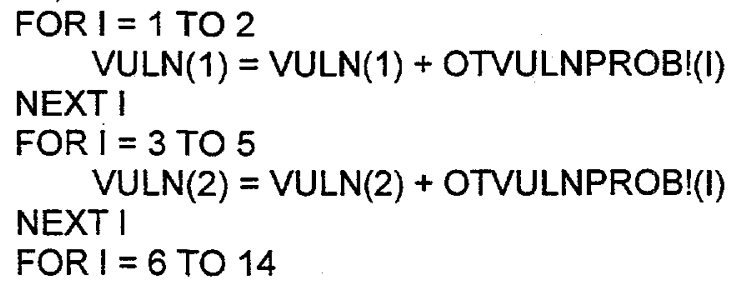


NEXT I

$$
\operatorname{VULN}(3)=\text { VULN(3) + OTVULNPROB!(I) }
$$

FOR I = 15 TO 101

$$
\text { VULN(4) = VULN(4) + OTVULNPROB!(I) }
$$

\section{NEXT I}

print \#1,

PRINT \#1, USING c\$; "For Vulnerability category Negligible, the probability is ";VULN(1)

PRINT \#1, USING c\$; "For Vulnerability category Low, the probability is ";VULN(2)

PRINT \#1, USING c\$; "For Vulnerability category High, the probability is ";VULN(3)

PRINT \#1, USING C\$; "For Vulnerability category Very High, the probability is "; VULN(4) print \#1,

RETURN

- Subroutine 5100 computes the Likelihood matrix probability values and

- accumulates them into the five Likelihood category probabilities

5100

FOR I $=1$ TO 4

FOR $\mathrm{J}=1 \mathrm{TO} 4$

LMAT! $(I, J)=$ THREAT $(\mathrm{l})$.$* VULN (\mathrm{J})$

NEXT I NEXT J

LHOOD! $(1)=$ LMAT! $(1,1)+\operatorname{LMAT}(1,2)+\operatorname{LMAT} !(1,3)+\operatorname{LMAT} !(2,1)$

LHOOD! $(2)=\operatorname{LMAT}(1,4)+\operatorname{LMAT} !(2,2)+\operatorname{LMAT}(3,1)+\operatorname{LMAT}(4,1)$

$\operatorname{LHOOD!}(3)=\operatorname{LMAT} !(2,3)+\operatorname{LMAT} !(3,2)+\operatorname{LMAT} !(4,2)$

LHOOD! $(4)=$ LMAT $!(2,4)+\operatorname{LMAT!}(3,3)+\operatorname{LMAT} !(4,3)$

LHOOD! $(5)=$ LMAT! $(3,4)+$ LMAT! $(4,4)$

PRINT \#1,

PRINT \#1, USING c\$; "Probability for Likelihood Category Negligible = "; LHOOD!(1)

PRINT \#1, USING c\$; "Probability for Likelihood Category Low = "; LHOOD!(2)

PRINT \#1, USING c\$; "Probability for Likelihood Category Medium = "; LHOOD!(3)

PRINT \#1, USING C\$; "Probability for Likelihood Category High = "; LHOOD!(4)

PRINT \#1, USING c\$; "Probability for Likelihood Category Very High = "; LHOOD!(5)

PRINT \#1,

\section{RETURN}

' Subroutine 5200 solicits input from the evaluator for the Consequence

' measure, given as weights/probabilities assigned to each of the five

- Consequence categories. This input is echoed to output. Finally,

' Risk Matrix probabilities are calculated and then accumulated into

' the five Risk category probabilities

\section{CLS}

PRINT "Enter estimate of site/facility diversion consequences."

PRINT "Enter five weights/probabilities associated with the categories:"

PRINT " Negligible = Fission products or other non-weapons material"

PRINT " (IAEA Attractiveness Level E or less)"

PRINT "'!

PRINT " Low = Small amounts of difficult to convert materials"

PRINT " $\quad$ (IAEA Attractiveness Level D, Category IV)"

PRINT "' 


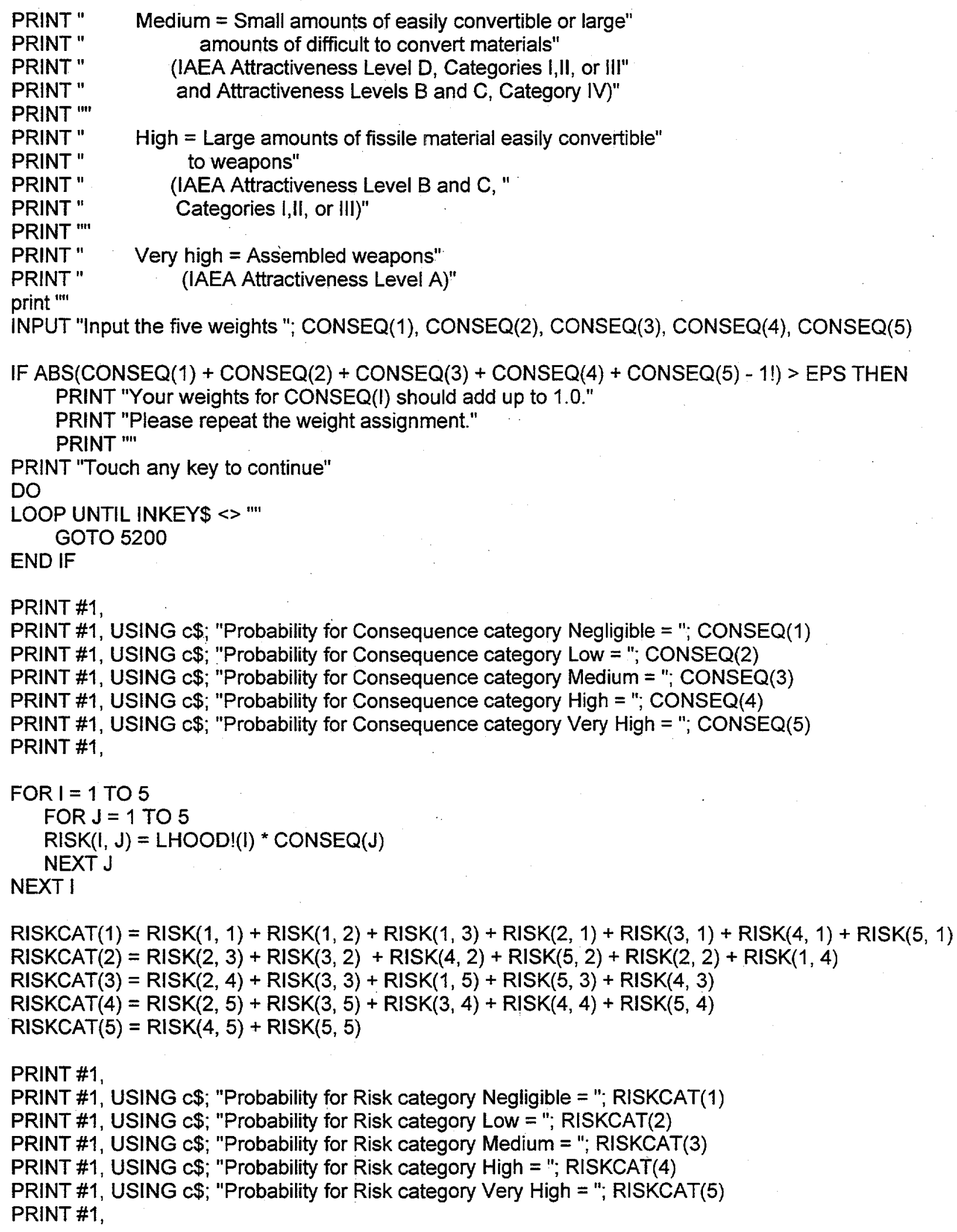

PRINT \#1,

PRINT \#1, USING c\$; "Probability for Risk category Negligible = "; RISKCAT(1)

PRINT \#1, USING c\$; "Probability for Risk category Low = "; RISKCAT(2)

PRINT \#1, USING C\$; "Probability for Risk category Medium = "; RISKCAT(3)

PRINT \#1, USING c\$; "Probability for Risk category High = "; RISKCAT(4)

PRINT \#1, USING c\$; "Probability for Risk category Very High = "; RISKCAT(5)

PRINT \#1, 


\section{レールー}

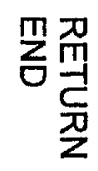

ơ 


\title{
Source code for the State Transfer Scenario
}

\author{
This is a prototype, with a text-based user interface. It implements \\ the proposed threat/vulnerability measure aggregation scheme, \\ including handling of evaluator uncertainty \\ The program will begin displaying the relevant threat and \\ vulnerability sub-measures one by one \\ and prompting the evaluator for his/her estimates of the weights (or \\ probabilities) to be assigned to each of the possible sub-measure \\ values. For each sub-measure, these weights should sum to 1 . The \\ program will solicit weights for the consequence measure, which \\ is based on the quantity and attractiveness of the nuclear materials \\ available for diversion at the site. \\ When the input for all relevant sub-measures has been entered, the \\ program will compute the aggregate values, will assign categorical \\ values to the threat and vulnerability measures, and will compute the \\ corresponding diversion risk measure. The evaluator-assigned \\ uncertainties will be propagated all the way through to the \\ diversion risk measure.
}

\section{DEFSNG A-H, O-Z \\ DEFINT I-N \\ OPTION BASE 1}

$\mathrm{EPS}=.001$

DIM X1(3) ' Long Standing Conflict - WEIGHTS

DIM X2(2) 'Political/ideological motivations - WEIGHTS

DIM X3(3) 'Religious motivations -- WEIGHTS

DIM X4(2) 'Perception of target vulnerability - WEIGHTS

DIM X5(4) 'Availability of resources - WEIGHTS

DIM X6(3) ' Training and leadership -- WEIGHTS

DIM X7(2) ' Knowledge and intelligence -- WEIGHTS

DIM X8(3) 'Site/facility socio-economic conditions - WEIGHTS

DIM Y1(3) ' Material surveillance -- WEIGHTS

DIM Y2(3) ' Transfer controls -- WEIGHTS

DIM Y3(3). ' 'Protective forces - WEIGHTS

DIM Y4(3) ' Intrusion detection - WEIGHTS

DIM Y5(3) ' Barriers/delays -- WEIGHTS

DIM Y6(3) 'Access controls - WEIGHTS

DIM Y7(2) ' Site location - WEIGHTS 


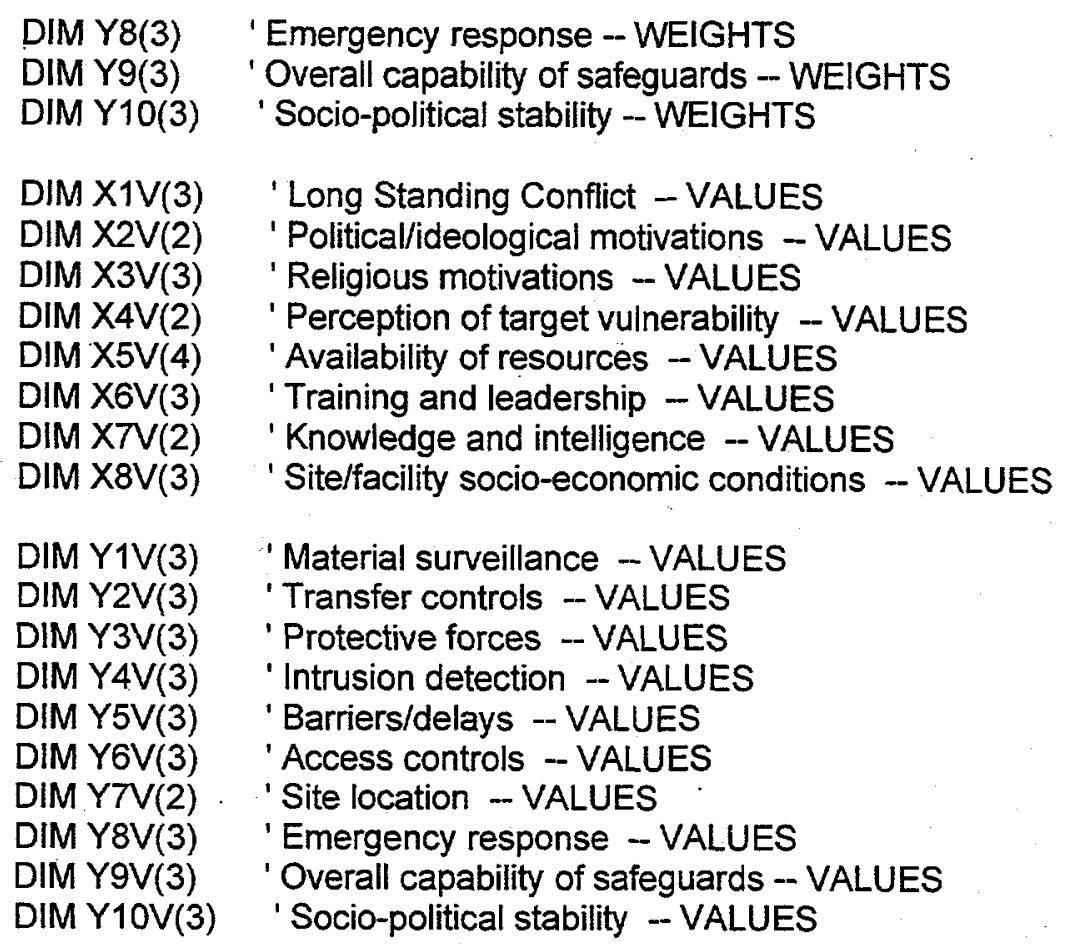

FOR I = 1 TO 3: READ X1V(I): NEXT I DATA $0.5,1.0,2.0$

FOR I = 1 TO 2: READ X2V(I): NEXT I DATA $0.5,1.0$

FOR I = 1 TO 3: READ X3V(l): NEXT I DATA $0.5,1.0,2.0$

FOR I = 1 TO 2: READ X4V(I): NEXT I DATA $0.5,1.5$

FOR I = 1 TO 4: READ X5V(I): NEXT I DATA 0.0, 0.5, 1.0, 2.0

FOR I = 1 TO 3: READ X6V(I): NEXT I DATA 0.5, 1.0, 2.0

FOR I = 1 TO 2: READ X7V(I): NEXT I DATA $0.5,1.5$

FOR I = 1 TO 3: READ X8V(I): NEXT I DATA $0.75,1.0,1.5$

FOR I = 1 TO 3: READ Y1V(I): NEXT I DATA $0.5,1.0,2.0$

FOR I = 1 TO 3: READ Y2V(I): NEXT I DATA $0.5,1.0,2.0$

FOR I = 1 TO 3: READ Y3V(I): NEXT I DATA $0.5,1.0,2.0$

FOR I = 1 TO 3: READ Y4V(l): NEXT I DATA $0.5,1.0,2.0$

FOR I = 1 TO 3: READ Y5V(l): NEXT I DATA 0.5, 1.0, 2.0

FOR I = 1 TO 3: READ YGV(I): NEXT I

DATA 0.5, 1.0, 2.0 
FOR I = 1 TO 2: READ Y7V(I): NEXT I

DATA $0.5,1.5$

FOR I = 1 TO 3: READ Y8V(I): NEXT I

DATA $0.5,1.0,2.0$

FOR I = 1 TO 3: READ Y9V(I): NEXT I

DATA $0.5,1.0,2.0$

FOR I = 1 TO 3: READ Y 1OV(I): NEXT I

DATA $0.5,1.0,2.0$

DIM STTHREATPROB!(101)

DIM STVULNPROB!(101)

DIM THREAT(4) 'Threat category probabilities

DIM VULN(4) 'Vulnerability category probabilities

DIM LHOOD!(5) 'Likelihood category probabilities

DIM LMAT! $(4,4)$ 'Likelihood matrix probabilities

DIM CONSEQ(5) 'Consequence category probabilities

DIM RISK $(5,5)$ 'Risk matrix probabilities

DIM RISKCAT(5) 'Risk category probabilities

DIM scentype\$(4) ' Scenario type names

FOR I = 1 TO 4: READ scentype\$(I): NEXT I

DATA "Insider theft", "Outsider theft", "Outsider attack", "State transfer"

INPUT "Enter name of output file "; outfile\$

OPEN outfile\$ FOR OUTPUT AS \#1

INPUT "Enter site/facility name "; sitename\$

' Define printout formats

$a 2 \$=" \& \# . \# \& \#$

a3\$ $=$ "\&\#.\#\# \&\#.\#\#\&\#.\#"

a4\$ $=$ "\&\#.\#\# \&\#.\#\#\&\#.\#\# \&\#.\#"

tv\$ = "\& \#\#\# \&\#.\#"

$\mathrm{C} \$=" \& \#$

lCS $=$ "\&\# \&\#.\#"

PRINT \#1,

PRINT \#1, "Evaluation of site/facility: "; sitename\$

PRINT \#1, "For scenario type: $\quad$ "; scentype\$(4)

PRINT \#1, "Output file name: "; outfile\$

PRINT \#1, "Program and Version: Version 4 of ST_EVAL"

PRINT \#1,

GOSUB 4000

STOP 
' Subroutines 110 through 290 solicit evaluator input for Threat and

' Vulnerability sub-measures. Subroutine 100 explains that the Threat

' and Vulnerability sub-measures are all treated as random variables,

' requiring that probabilities (or weights) summing to 1 must be assigned

' to each of the numerical values taken on by that sub-measure.

100 CLS

PRINT "The Threat and Vulnerability sub-measures used in this scheme"

PRINT "for estimating the risk of diversion of nuclear materials are"

PRINT "all treated as random variables, requiring that probabilities"

PRINT "(or weights) summing to 1 must be assigned to each of the"

PRINT "numerical values taken on by that sub-measure. The input routines"

PRINT "will enforce this requirement."

PRINT "'

PRINT "In addition, the assignment of probabilities to the sub-measure"

PRINT "can be used to represent the evaluators uncertainty about the"

PRINT "correct value of the sub-measure. For instance, in the absence of"

PRINT "any information about the true value of the sub-measure"

PRINT "(i.e., complete uncertainty), the evaluator should assign equal"

PRINT "weights to all of the sub-measure values."

PRINT "'"

PRINT "Touch any key to continue"

DO

LOOP UNTIL INKEY\$ $<>$ '"'

RETURN

110 CLS

PRINT "Existence of a Long Standing Conflict "

PRINT "'"

PRINT " $X 1=0.5$, no underlying conflict"

PRINT " X_1 $=1.0$, recently developed conflict (decades-long duration)"

PRINT " X_1 $=2.0$, long standing conflict (centuries-long duration)"

PRINT "m

PRINT "*Long Standing Conflict* is shorthand for aspects of intensity "

PRINT "and long duration in the motivation of the threat groups. It can "

PRINT "derive from extreme religious prejudice, language/cultural "

PRINT "differences, and/or from a long history of oppression, "

PRINT "suppression, or warfare between two groups of people. Examples "

PRINT "include Catholic Irish vs. Irish Protestants and the British "

PRINT "or the Basques vs. the Spanish or Islamic fundamentals vs. "

PRINT "secular Moslem governments. "

PRINT '"'

INPUT "Input three $X \_1$ weights "; $X 1(1), X 1(2), X 1(3)$

IF ABS $(X 1(1)+X 1(2)+X 1(3)-1$ !) $>$ EPS THEN

PRINT "Your weights for $X \_1$ should add up to 1.0."

PRINT "Please repeat the weight assignment."

PRINT "Touch any key to continue"

DO

LOOP UNTIL INKEY\$ <> "'!

GOTO 110

END IF 
PRINT \#1, "Variable $X 1$ is Existence of a Long Standing Conflict"

PRINT \#1, USING a3\$; "X_1 values are "; X1V(1); " "; X1V(2); " "; X1V(3)

PRINT \#1, USING a3\$; "X_1 weights are "; X1(1);" "; X1(2); " "; X1(3)

PRINT \#1,

RETURN

120 CLS

PRINT "Political//deological Motivations "

PRINT "'"

PRINT " X_2 $=0.5$, no political/ideological basis "

PRINT " $X 2=1.0$, political/ideological motivations"

PRINT "'!

PRINT "Examples would be support for terrorism as a component "

PRINT "of the cold war struggle between Communism and the West."

PRINT "'r

INPUT "Input two X_2 weights "; X2(1), X2(2)

IF ABS $(X 2(1)+X 2(\overline{2})-1$ !) > EPS THEN

PRINT "Your weights for $X \_2$ should add up to 1.0."

PRINT "Please repeat the weight assignment."

PRINT "Touch any key to continue"

DO

LOOP UNTIL INKEY\$ <> "'"

GOTO 120

END IF

PRINT \#1, "Variable X2 is Political/ldeological Motivations"

PRINT \#1, USING a2\$; "X_2 values are "; X2V(1); " "; X2V(2)

PRINT \#1, USING a2\$; "X_2 weights are "; X2(1); " "; X2(2)

PRINT \#1,

\section{RETURN}

130 CLS

PRINT "Religious Motivations "

PRINT"

PRINT " $\quad X 3=0.5$, no religious animosity"

PRINT " $\quad X-3=1.0$, some religious animosity"

PRINT " $X-3=2.0$, strong religious animosity"

PRINT '"'

PRINT "The intent here is to capture aspects of motivation distinct "

PRINT "from possible religious components of the "; blood; feud; " measure "

PRINT "above."

PRINT '":"

INPUT "Input three X_3 weights "; X3(1), X3(2), X3(3)

IF ABS $(X 3(1)+X 3(2)+X 3(3)-1$ !) $>$ EPS THEN

PRINT "Your weights for $X \_3$ should add up to 1.0."

PRINT "Please repeat the weight assignment."

PRINT "Touch any key to continue"

DO

LOOP UNTIL INKEY\$ $<>$ '"'

GOTO 130 
END IF

PRINT \#1, "Variable X3 is Religious Motivations"

PRINT \#1, USING a3\$; "X_3 values are "; X3V(1); " "; X3V(2); " "; X3V(3)

PRINT \#1, USING a3\$; "X_3 weights are "; X3(1); " "; X3(2); " "; X3(3)

PRINT \#1,

RETURN

140 CLS

PRINT "Perception of target vulnerability "

PRINT "'!

PRINT " X_4 $=0.5$, facility not perceived as vulnerable "

PRINT " X_4 $=1.5$, facility perceived as vulnerable"

PRINT "m!

PRINT "The reasoning here is that, even for a threat group predisposed "

PRINT "to action, if a particular facility is perceived as not vulnerable, "

PRINT "they will direct their attention elsewhere."

PRINT 'm!

INPUT "Input two $X \_4$ weights "; $X 4(1), X 4(2)$

IF ABS $(X 4(1)+X 4(\overline{2})-1$ !) $>$ EPS THEN

PRINT "Your weights for $X \_4$ should add up to 1.0."

PRINT "Please repeat the weight assignment."

PRINT "Touch any key to continue"

DO

LOOP UNTIL INKEY\$ <> "'

GOTO 140

END IF

PRINT \#1, "Variable X4 is Perception of Target Vulnerability"

PRINT \#1, USING a2\$; "X_4 values are "; X4V(1); " "; X4V(2)

PRINT \#1, USING a2\$; "X_4 weights are "; X4(1); " "; X4(2)

PRINT \#1,

\section{RETURN}

150 CLS

PRINT "Availability of resources "

PRINT "'"

PRINT " X_5 $=0.0$, no resources available"

PRINT" X_5 $=0.5$, modest, locally collected resources"

PRINT " X_5 = 1.0, intermediate, state-supported"

PRINT " X_5 = 2.0, large, state-supported"

PRINT "'!

PRINT "This is a measure of the resource base of the threat group."

PRINT "'

INPUT "Input four $X \_5$ weights "; $X 5(1), X 5(2), X 5(3), X 5(4)$

IF ABS $(X 5(1)+X 5(2)+X 5(3)+X 5(4)-1$ !) $>$ EPS THEN

PRINT "Your weights for $X \_5$ should add up to 1.0."

PRINT "Please repeat the weight assignment."

PRINT "Touch any key to continue"

DO

LOOP UNTIL INKEY\$ <> "'"

GOTO 150 


\section{END IF}

PRINT \#1, "Variable X5 is Availability of Threat Group Resources"

PRINT \#1, USING a4\$; "X_5 values are "; X5V(1); " "; X5V(2); " "; X5V(3); " "; X5V(4)

PRINT \#1, USING a4\$; "X_5 weights are "; X5(1); " "; X5(2); " "; X5(3); " "; X5(4)

PRINT \#1,

\section{RETURN}

160 CLS

PRINT "Training and leadership "

PRINT "'!

PRINT " $X \_6=0.5$, poor training and leadership"

PRINT " X_6 $6=1.0$, intermediate training and leadership"

PRINT " X_6 $=2.0$, well-trained and led"

PRINT "'!

PRINT "This is a measure of the quality of training and leadership "

PRINT "of the threat organization."

PRINT "'"

INPUT "Input three X_6 weights "; X6(1), X6(2), X6(3)

IF ABS(X6(1) + X6(2) +X6(3) - 1!) > EPS THEN

PRINT "Your weights for X_6 should add up to 1.0."

PRINT "Please repeat the weight assignment."

PRINT "Touch any key to continue"

DO

LOOP UNTIL INKEYS <> "'"

GOTO 160

\section{END IF}

PRINT \#1, "Variable X6 is Threat Group Training and Leadership"

PRINT \#1, USING a3\$; "X_6 values are "; X6V(1); " "; X6V(2); " "; X6V(3)

PRINT \#1, USING a3\$; "X_6 weights are "; X6(1); " "; X6(2); " "; X6(3)

PRINT \#1,

\section{RETURN}

170 CLS

PRINT "Knowledge and intelligence"

PRINT "'"

PRINT " X_7 $=0.5$, poor intelligence capabilities"

PRINT " X_7 =1.5, good intelligence capabilities"

PRINT "m

PRINT "This is a measure of the access of the threat organization "

PRINT "to information about the potential target and their ability "

PRINT "to use that information."

PRINT "'!

INPUT "Input two $X \_7$ weights "; $X 7(1), X 7(2)$

IF ABS $(X 7(1)+X 7(\overline{2})-1$ !) $>$ EPS THEN

'PRINT "Your weights for $X \_7$ should add up to 1.0."

PRINT "Please repeat the weight assignment."

PRINT "Touch any key to continue"

DO

LOOP UNTIL INKEY\$ $<>$ '"'

GOTO 170 
END IF

PRINT \#1, "Variable $X 7$ is Threat Group Knowledge and Intelligence Capability"

PRINT\#1, USING a2\$; "X_7 values are "; X7V(1);" "; X7V(2)

PRINT \#1, USING a2\$; "X_7 weights are "; $X 7(1)$; " "; $X 7(2)$

PRINT \#1,

\section{RETURN}

180 CLS

PRINT "Site/facility socio-economic conditions "

PRINT "'"

PRINT " X_8 $=0.75$, good conditions"

PRINT " X_8 $=1.0$, not-so-good"

PRINT " X_8 $=1.5$, extremely difficult conditions"

PRINT "'!

PRINT "This attempts to measure the likelihood that the threat "

PRINT "organization can subvert/coopt insiders to support its "

PRINT "diversion attempt or that insiders will themselves attempt diversion."

PRINT "'!

INPUT "Input three $X \_8$ weights "; $X 8(1), X 8(2), X 8(3)$

IF ABS $(X 8(1)+X 8(2)+X 8(3)-1$ ! $)>$ EPS THEN

PRINT "Your weights for $X \_8$ should add up to 1.0."

PRINT "Please repeat the weight assignment."

PRINT "Touch any key to continue"

DO

LOOP UNTIL INKEY\$ <> "'

END IF

GOTO 180

PRINT \#1, "Variable X8 is Site/Facility Socio-Economic Conditions"

PRINT \#1, USING a3\$; "X_8 values are "; X8V(1); " "X8V(2); " "; X8V(3)

PRINT \#1, USING a3\$; "X_8 weights are "; X8(1); " "; X8(2); " "; X8(3)

PRINT \#1,

\section{RETURN}

210 CLS

PRINT "Material Surveillance "

PRINT "'!

PRINT " Y Y 1 $=0.5$, excellent system of material surveillance"

PRINT " $Y_{-}^{-1}=1.0$, good system of material surveillance"

PRINT " $Y_{-1}^{-1}=2.0$, poor system of material surveillance"

PRINT "'"

PRINT "This measures the material accounting and surveillance "

PRINT "infrastructure that enables facility management to detect "

PRINT "attempted or successful diversion."

PRINT "'"

INPUT "Input three $Y_{-} 1$ weights "; $Y 1(1), Y 1(2), Y 1(3)$

IF ABS $(Y 1(1)+Y 1(2)+Y 1(3)-1$ !) $>$ EPS THEN

PRINT "Your weights for $Y_{-} 1$ should add up to 1.0."

PRINT "Please repeat the weight assignment."

PRINT "Touch any key to continue" 
DO

LOOP UNTIL INKEY\$ <> "'" GOTO 210

END IF

PRINT \#1, "Variable Y1 is Site Material Surveillance"

PRINT \#1, USING a3\$; "Y_1 values are "; Y1V(1); " "; Y1V(2); " "; Y1V(3)

PRINT \#1, USING a3\$; "Y_1 weights are "; Y1(1); " "; Y1(2); " "; Y1(3)

PRINT \#1,

\section{RETURN}

220 CLS

PRINT "Transfer Controls "

PRINT "'"

PRINT " Y_2 $=0.5$, excellent system of transfer control"

PRINT " $Y-2=1.0$, good system of transfer control"

PRINT " $Y_{-2}=2.0$, poor system of transfer control"

PRINT "'!'

PRINT "This measures the security of material in transit from one "

PRINT "storage/utilization location to another."

PRINT "'"

INPUT "Input three Y_2 weights "; Y2(1), Y2(2), Y2(3)

IF ABS $(Y 2(1)+Y 2(2)+Y 2(3)-1 !)>$ EPS THEN

PRINT "Your weights for Y_2 should add up to 1.0."

PRINT "Please repeat the weight assignment."

PRINT "Touch any key to continue"

DO

LOOP UNTIL INKEY\$ <> "'"

GOTO 220

END IF

PRINT \#1, "Variable Y2 is Site Transfer Controls"

PRINT \#1, USING a3\$; "Y_2 values are "; Y2V(1); " "; Y2V(2); " "; Y2V(3)

PRINT \#1, USING a3\$; "Y_2 weights are "; Y2(1); " "; Y2(2); " "; Y2(3)

PRINT \#1,

\section{RETURN}

230 CLS

PRINT "Protective Forces"

PRINT "'"'

PRINT " Y_3 $=0.5$, excellent protective forces"

PRINT " Y_3 $3=1.0$, good protective forces"

PRINT " $Y_{-3}=2.0$, poor protective forces"

PRINT '"'

PRINT "This is a measure of the ability of the facility guard forces "

PRINT "to prevent or delay a diversion."

PRINT '"'

INPUT "Input three Y_3 weights "; Y3(1), Y3(2), Y3(3)

IF ABS(Y3(1) + Y3(2) + Y3(3) - 1!) > EPS THEN

PRINT "Your weights for $Y_{-} 3$ should add up to 1.0."

PRINT "Please repeat the weight assignment."

PRINT "Touch any key to continue" 
DO

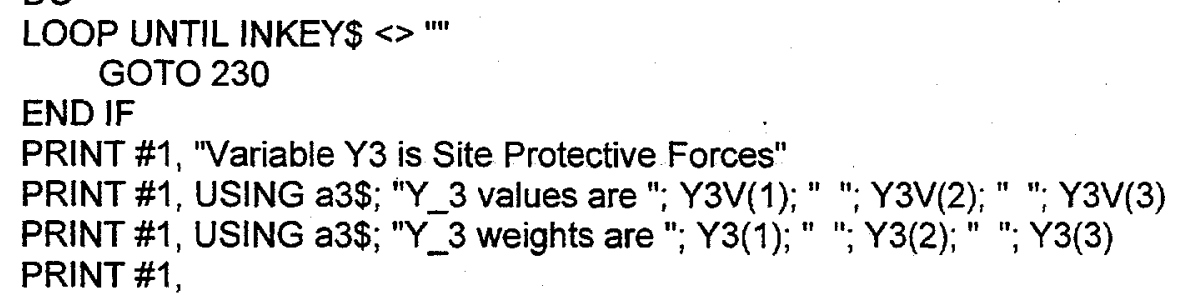

\section{RETURN}

250 CLS

PRINT "Barriers/Delays "

PRINT '"'

PRINT " Y $5=0.5$, excellent barriers/delays"

PRINT " Y_5 $=1.0$, good barriers/delays"

PRINT " Y $5=2.0$, poor barriers/delays"

PRINT "

PRINT "This measures facility design and operation features that "

PRINT "tend to delay a diversion attempt long enough for response "

PRINT "of off-site protection forces."

PRINT "'"

INPUT "Input three $Y \_5$ weights "; $Y 5(1), Y 5(2), Y 5(3)$

IF ABS $(Y 5(1)+Y 5(2)+Y 5(3)-1$ !) > EPS THEN

PRINT "Your weights for $Y \_5$ should add up to 1.0."

PRINT "Please repeat the weight assignment." 


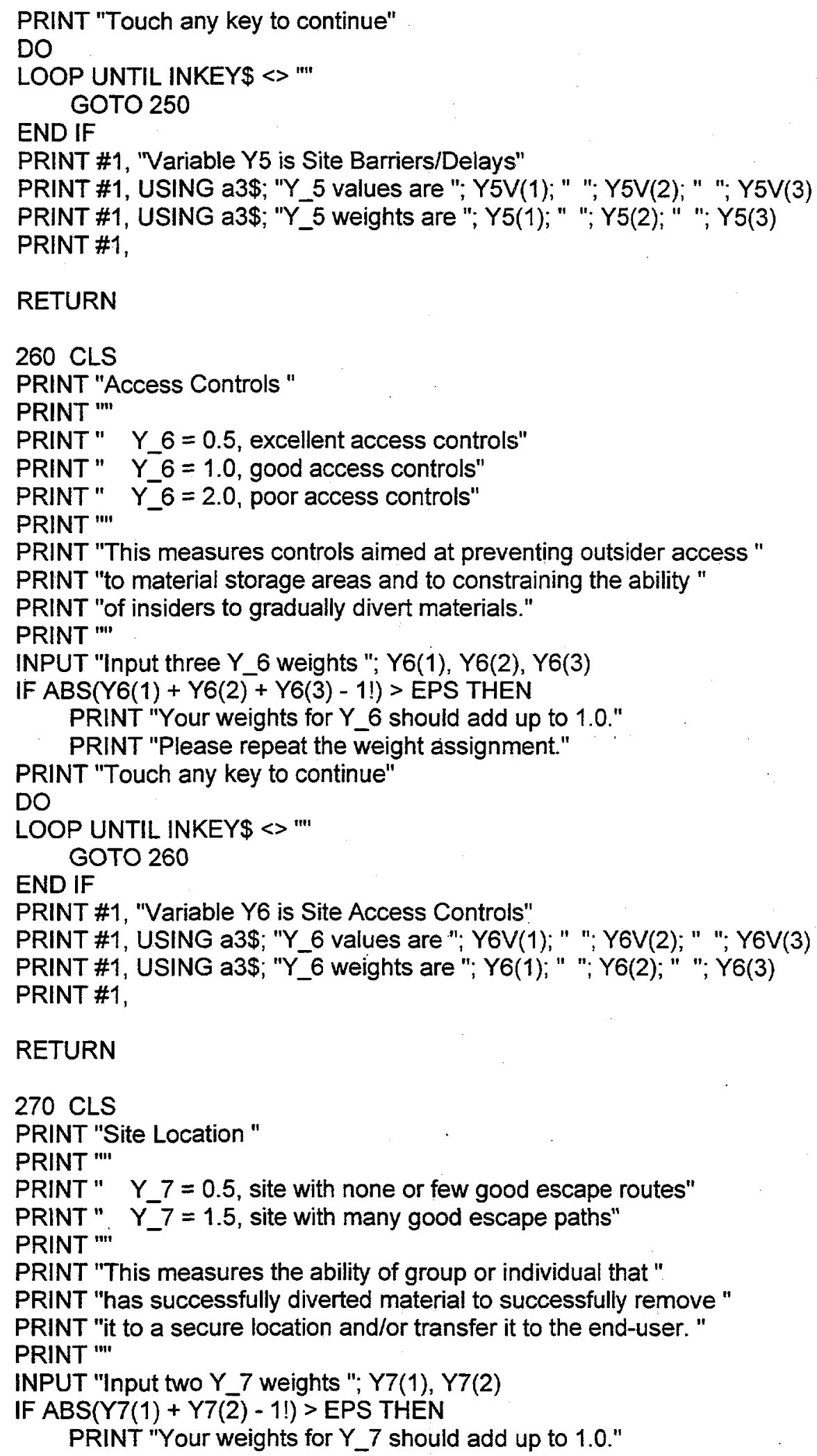


PRINT "Please repeat the weight assignment."

PRINT "Touch any key to continue"

DO

LOOP UNTIL INKEY\$ <> '"'

END IF

GOTO 270

PRINT \#1, "Variable Y7 is Site Location (as it affects interdiction of "

PRINT \#1, " diverted materials)"

PRINT \#1, USING a2\$; "Y_7 values are "; Y7V(1); " "; YTV(2)

PRINT \#1, USING a2\$; "Y_7 weights are "; Y7(1); " "; $Y 7(2)$

PRINT \#1,

\section{RETURN}

\section{CLS}

PRINT "Emergency Response "

PRINT "m

PRINT " Y_8 $=0.5$, site with excellent emergency response resources"

PRINT" $Y$ _ $8=1.0$, site with some emergency response"

PRINT" $Y \_8=2.0$, site with none or poor emergency response"

PRINT "'"

PRINT "This measures the ability of off-site emergency response to "

PRINT "interdict a forcible diversion in progress, given timely "

PRINT "notification. Note that measures $Y_{-} 4, Y_{-} 5$, and $Y_{-} 7$ also "

PRINT "condition the likelihood of successful interdiction of a "

PRINT "diversion in progress. "

PRINT '"'

INPUT "Input three $Y \_8$ weights "; $Y 8(1), Y 8(2), Y 8(3)$

IF ABS(Y8(1) + Y8(2) + Y8(3) - 1!) > EPS THEN

PRINT "Your weights for $Y_{-} 8$ should add up to 1.0."

PRINT "Please repeat the weight assignment."

PRINT "Touch any key to continue"

DO

LOOP UNTIL INKEY\$ <> "'"

GOTO 280

END IF

PRINT \#1, "Variable Y8 is Site Emergency Response (as it affects "

PRINT \#1, " interdiction of diverted materials)"

PRINT \#1, USING a3\$; "Y_8 values are "; Y8V(1); " "; Y8V(2); " "; Y8V(3)

PRINT \#1, USING a3\$; "Y_8 weights are "; Y8(1); " "; Y8(2); " "; Y8(3)

PRINT \#1,

\section{RETURN}

285 CLS

PRINT "Overall Capability of Host Country Safeguards Measures"

PRINT "'"

PRINT " Y_9 9.5 , Robust safeguards measures "

PRINT" Y_ $9=1.0$, Safeguards with some weaknesses in design "

PRINT " $\quad$ or implementation "

PRINT " $Y_{-} 9=2.0$, Weak or non-existent safeguards "

PRINT "' 
PRINT "This measures the overall capability of safeguards measures "

PRINT "to detect/alarm attempted state transfer of weapons material "

PRINT "to unauthorized state- or sub-state-organizations."

PRINT '"'

INPUT "Input three $Y \_9$ weights "; $Y 9(1), Y 9(2), Y 9(3)$

IF ABS $(Y 9(1)+Y 9(2)+Y 9(3)-1$ !) > EPS THEN

PRINT "Your weights for $Y_{-}$9 should add up to 1.0."

PRINT "Please repeat the weight assignment."

PRINT "Touch any key to continue"

DO

LOOP UNTIL INKEY\$ <> "'"

GOTO 285

END IF

PRINT \#1, "Variable Y9 is Overall Capability of Host Country Safeguards"

PRINT \#1, USING a3\$; "Y_9 values are "; Y9V(1); " "; Y9V(2); " "; Y9V(3)

PRINT \#1, USING a3\$; "Y_9 weights are "; Y9(1); " "; Y9(2); " "; Y9(3)

PRINT \#1,

\section{RETURN}

290 CLS

PRINT "Socio-political stability "

PRINT "'"

PRINT " $Y_{-} 10=0.5$, stable government with good foreign "

PRINT " relations, checks and balances"

PRINT " Y $\quad 10=1.0$, some instability, some foreign enemies, "

PRINT " some checks and balances"

PRINT " Y Y $10=2.0$, significant instability, many factions, "

PRINT " many enemies, no checks and balances"

PRINT "'"

PRINT "This attempts to measure the likelihood that all or part of "

PRINT "the relevant government organizations will formulate and "

PRINT "implement or will fail to impede a state transfer diversion "

PRINT "attempt. "

PRINT "'?

INPUT "Input three $Y \_10$ weights "; $Y 10(1), Y 10(2), Y 10(3)$

IF ABS $(Y 10(1)+Y 10(2)+Y 10(3)-1$ !) > EPS THEN

PRINT "Your weights for $Y_{-} 10$ should add up to 1.0."

PRINT "Please repeat the weight assignment."

PRINT "Touch any key to continue"

DO

LOOP UNTIL INKEY\$ <> "'"

GOTO 290

END IF

PRINT \#1, "Variable $Y 10$ is Host Country Socio-political Stability"

PRINT \#1, USING a3\$; "Y_10 values are "; Y10V(1); " "; Y10V(2); " "; Y10V(3)

PRINT \#1, USING a3\$; "Y_10 weights are "; Y10(1); " "; Y10(2); " "; Y10(3)

PRINT \#1,

\section{RETURN}

- Subroutine 4000 calculates Threat and Vulnerability Probabilities 
' for the State Transfer Theft scenario type

4000 GOSUB 100

GOSUB 140

GOSUB 150

GOSUB 160

GOSUB 170

GOSUB 180

GOSUB 285

GOSUB 290

DIM STTHREATV! $(2,4,3,2,3)$

DIM STTHREATP! $(2,4,3,2,3)$

DIM STVULNV! $(3,3)$

DIM STVULNP! $(3,3)$

FOR I $=1$ TO 2

FOR J $=1$ TO 4

FOR K $=1$ TO 3

FOR $L=1$ TO 2

FOR $M=1$ TO 3

STTHREATV! $(I, J, K, L, M)=X 4 V(l) * X 5 V(J) * X 6 V(K) * X 7 V(L) * X 8 V(M)$

STTHREATP! $(\mathrm{I}, \mathrm{J}, \mathrm{K}, \mathrm{L}, \mathrm{M})=\mathrm{X} 4(\mathrm{I}){ }^{*} \mathrm{X}(\mathrm{J}){ }^{*} \mathrm{X} 6(\mathrm{~K}) * \mathrm{X} 7(\mathrm{~L}) * \mathrm{X} 8(\mathrm{M})$

STTHREATPROB!((STTHREATV! $\left.\left.(I, J, K, L, M){ }^{*} 7\right)+1\right)$ = STTHREATPROB!((STTHREATV!(I, J, K, $L, M) * 7)+1)+$ STTHREATP! $(I, J, K, L, M)$

' PRINT \#1, "STTHREATV!(-,-,-,"; l; ","; J; ","; K; ","; L; ","; M; ") = "; STTHREATV!(I, J, K, L, M)

' PRINT \#1, "STTHREATP!(-,-,-,"; I; ","; J; ","; K; ","; L; ","; M; ") = "; STTHREATP!(I, J, K, L, M)

'PRINT \#1,

NEXTM

NEXT L

NEXT K

NEXT J

NEXT I

FOR I = 1 TO 3

FOR $\mathrm{J}=1$ TO 3

STVULNV!(l,J) $=Y 9 V(I)^{\star} Y 10 V(J)$

STVULNP! $(1, \mathrm{~J})=\mathrm{Y} 9(\mathrm{I})^{*} \mathrm{Y} 10(\mathrm{~J})$

STVULNPROB!((STVULNV!(I,J) * 24) + 1) = STVULNPROB!((STVULNV! $(1, J) * 24)+1)+$ STVULNP! $(I, J)$

- PRINT \#1, "STVULNV!("; l;",";J; ") = "; STVULNV!(I,J)

, PRINT \#1, "STVULNP!("; l;",,;J; ") = "; STVULNP!(I,J)

- PRINT \#1,

NEXT J

NEXT I

' FOR I = 1 TO 101

' PRINT \#1, USING tu\$; "For Threat value = "; $(I-1) / 7$; " the probability is "; STTHREATPROB!(I)

' NEXT I

' PRINT \#1,

' FOR I = 1 TO 101

- PRINT \#1, USING tw\$; "For Vulnerability value = "; (I - 1)/24; " the probability is "; STVULNPROB!(l)

' NEXT'

GOSUB 5000

GOSUB 5100

GOSUB 5200 


\section{RETURN}

- Subroutine 5000 accumulates the Threat probabilities values into

' four Threat category probability values for the State Transfer

' scenario type; it also accumulates Vulnerability probabilities into the

' four Vulnerability category probability values for the State Transfer

' scenario type.

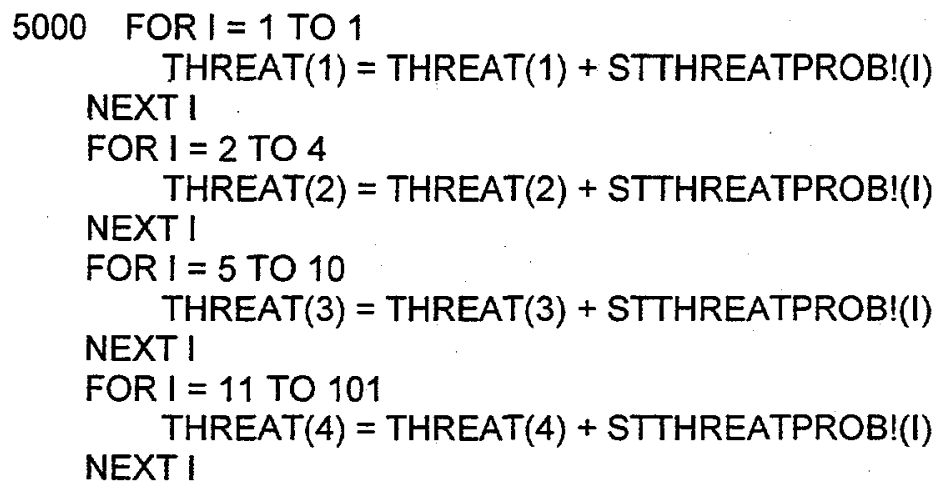

print \#1,

print \#1, using c\$; "For Threat category Negligible, the probability is ";THREAT(1)

print \#1, using $c \$$; "For Threat category Low, the probability is ";THREAT(2)

print \#1, using c\$; "For Threat category High, the probability is ";THREAT(3)

print \#1, using c\$; "For Threat category Very High, the probability is ";THREAT(4)

print \#1,

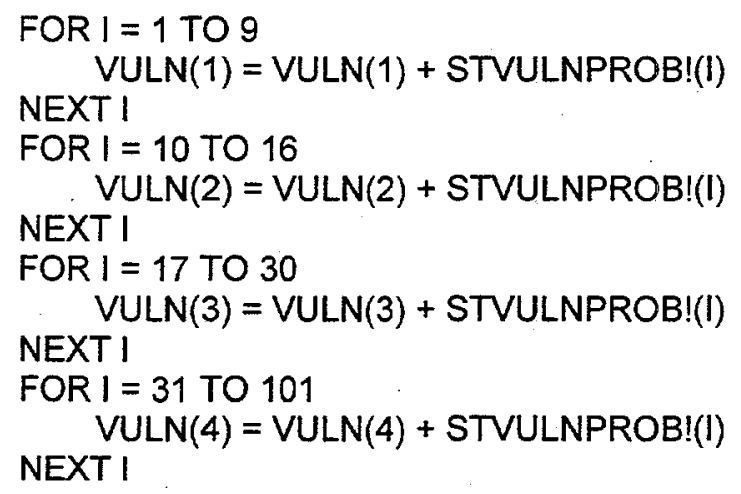

print \#1,

print \#1, using c\$; "For Vulnerability category Negligible, the probability is ";VULN(1)

print \#1, using c\$; "For Vulnerability category Low, the probability is ";VULN(2)

print \#1, using c\$; "For Vulnerability category High, the probability is ";VULN(3)

print \#1, using $c \$$; "For Vulnerability category Very High, the probability is ";VULN(4)

print \#1,

RETURN

- Subroutine 5100 computes the Likelihood matrix probability values and

' accumulates them into the five Likelihood category probabilities 
FOR $I=1 \mathrm{TO} 4$

FOR $\mathrm{J}=1$ TO 4

LMAT! $(I, J)=$ THREAT $(I) * \operatorname{VULN}(J)$ NEXT $J$

NEXT I

LHOOD! $(1)=$ LMAT! $(1,1)+$ LMAT! $(1,2)+$ LMAT! $(1,3)+$ LMAT! $(2,1)$

LHOOD! $(2)=\operatorname{LMAT} !(1,4)+\operatorname{LMAT} !(2,2)+\operatorname{LMAT} !(3,1)+\operatorname{LMAT} !(4,1)$

LHOOD! (3) = LMAT! $(2,3)+\operatorname{LMAT!}(3,2)+\operatorname{LMAT} !(4,2)$

LHOOD!(4) = LMAT! $(2,4)+\operatorname{LMAT!}(3,3)+\operatorname{LMAT} !(4,3)$

PRINT \#1,

LHOOD! $(5)=\operatorname{LMAT!}(3,4)+\operatorname{LMAT} !(4,4)$

PRINT \#1, USING c\$; "Probability for Likelihood category Negligible = "; LHOOD!(1)

PRINT \#1, USING c\$; "Probability for Likelihood category Low = "; LHOOD!(2)

PRINT \#1, USING c\$; "Probability for Likelihood category High = "; LHOOD!(3)

PRINT \#1, USING c\$; "Probability for Likelihood category Very High = "; LHOOD!(4)

PRINT \#1, USING c\$; "Probability for Likelihood category Very High = "; LHOOD!(5)

PRINT \#1,

RETURN

- Subroutine 5200 solicits input from the evaluator for the Consequence

' measure, given as weights/probabilities assigned to each of the five

' Consequence categories. This input is echoed to output. Finally,

- Risk Matrix probabilities are calculated and then accumulated into

' the five Risk category probabilities

5200 CLS

PRINT "Enter estimate of site/facility diversion consequences."

PRINT "Enter five weights/probabilities associated with the categories:"

PRINT "'!

PRINT " . Negligible $=$ Fission products or other non-weapons material"

PRINT " (IAEA Attractiveness Level E or less)"

PRINT "'"

PRINT " Low = Small amounts of difficult to convert materials"

PRINT " $\quad$ (IAEA Attractiveness Level D, Category IV)"

PRINT "'+

PRINT"

PRINT"

Medium = Small amounts of easily convertible or large" amounts of difficult to convert materials"

PRINT"

PRINT"

PRINT "'!

PRINT "

PRINT "

PRINT"

PRINT "

PRINT "'"

PRINT "

PRINT "

PRINT "'!

(IAEA Attractiveness Level D, Categories I,II, or III" and Attractiveness Levels B and C, Category IV)"

INPUT "Input the five weights "; CONSEQ(1), CONSEQ(2), CONSEQ(3), CONSEQ(4), CONSEQ(5)

IF ABS(CONSEQ(1) + CONSEQ(2) + CONSEQ(3) + CONSEQ(4) + CONSEQ(5) - 1!) > EPS THEN PRINT "Your weights for CONSEQ(l) should add up to 1.0." 
PRINT "Please repeat the weight assignment."

PRINT "'."

PRINT "Touch any key to continue"

DO

LOOP UNTIL INKEYS $<>$ '"'

END IF

GOTO 290

PRINT \#1,

PRINT \#1, USING c\$; "Probability for Consequence category Negligible = "; CONSEQ(1)

PRINT \#1, USING C\$; "Probability for Consequence category Low = "; CONSEQ(2)

PRINT \#1, USING c\$; "Probability for Consequence category Medium = "; CONSEQ(3)

PRINT \#1, USING C\$; "Probability for Consequence category High = "; CONSEQ(4)

PRINT \#1, USING c\$; "Probability for Consequence category Very High = "; CONSEQ(5)

PRINT \#1,

FOR I $=1$ TO 5

FOR $\mathrm{J}=1$ TO 5

RISK $(l, \mathrm{~J})=$ LHOOD! (I) * CONSEQ $(\mathrm{J})$

NEXT J

NEXT I

$\operatorname{RISKCAT}(1)=\operatorname{RISK}(1,1)+\operatorname{RISK}(1,2)+\operatorname{RISK}(1,3)+\operatorname{RISK}(2,1)+\operatorname{RISK}(3,1)+\operatorname{RISK}(4,1)+\operatorname{RISK}(5,1)$

$\operatorname{RISKCAT}(2)=\operatorname{RISK}(2,3)+\operatorname{RISK}(3,2)+\operatorname{RISK}(4,2)+\operatorname{RISK}(5,2)+\operatorname{RISK}(2,2)+\operatorname{RISK}(1,4)$

$\operatorname{RISKCAT}(3)=\operatorname{RISK}(2,4)+\operatorname{RISK}(3,3)+\operatorname{RISK}(1,5)+\operatorname{RISK}(5,3)+\operatorname{RISK}(4,3)$

$\operatorname{RISKCAT}(4)=\operatorname{RISK}(2,5)+\operatorname{RISK}(3,5)+\operatorname{RISK}(3,4)+\operatorname{RISK}(4,4)+\operatorname{RISK}(5,4)$

$\operatorname{RISKCAT}(5)=\operatorname{RISK}(4,5)+\operatorname{RISK}(5,5)$

PRINT \#1,

PRINT \#1, USING c\$; "Probability for Risk category Negligible = "; RISKCAT(1)

PRINT \#1, USING c\$; "Probability for Risk category Low = "; RISKCAT(2)

PRINT \#1, USING c\$; "Probability for Risk category Medium = "; RISKCAT(3)

PRINT \#1, USING c\$; "Probability for Risk category High = "; RISKCAT(4)

PRINT \#1, USING c\$; "Probability for Risk category Very High = "; RISKCAT(5)

PRINT \#1,

RETURN

END 


\section{Source code for the Transportation Diversion Scenario}

- This is a prototype, with a text-based user interface. It implements

- the proposed threat/vulnerability measure aggregation scheme,

- including handling of evaluator uncertainty

- The program will begin displaying the relevant threat and

- vulnerability sub-measures one by one

- and prompting the evaluator for his/her estimates of the weights (or

1 probabilities) to be assigned to each of the possible sub-measure

1 values. For each sub-measure, these weights should sum to 1 . The

- program will solicit weights for the consequence measure, which

' is based on the quantity and attractiveness of the nuclear materials

, available for diversion at the site.

When the input for all relevant sub-measures has been entered, the program will compute the aggregate values, will assign categorical values to the threat and vulnerability measures, and will compute the corresponding diversion risk measure. The evaluator-assigned uncertainties will be propagated all the way through to the diversion risk measure.

DEFSNG A-H, O-Z

DEFINT I-N

OPTION BASE 1

$E P S=.001$

DIM X1(3) ' Blood feud -- WEIGHTS

DIM X2(2) ' Political/ideological motivations - WEIGHTS

DIM X3(3) ' Religious motivations - WEIGHTS

DIM X4(2) ' Perception of target vulnerability -- WEIGHTS

DIM X5(4) 'Availability of resources - WEIGHTS

DIM X6(3) 'Training and leadership - WEIGHTS

DIM X7(2) 'Knowledge and intelligence - WEIGHTS

DIM X8(3) ' Site/facility socio-economic conditions - WEIGHTS

DIM Y1(3) ' Material surveillance -- WEIGHTS

DIM Y2(3) ' Transfer controls -- WEIGHTS

DIM Y3(3) 'Protective forces - WEIGHTS

DIM Y4(3) ' Intrusion detection -- WEIGHTS

DIM Y5(3) ' Barriers/delays - WEIGHTS

DIM Y6(3) ' Access controls -- WEIGHTS

DIM Y7(2) ' Site location - WEIGHTS

DIM Y8(3) ' Emergency response -- WEIGHTS

DIM Y9(3) ' 'Overall capability of safeguards -- WEIGHTS 


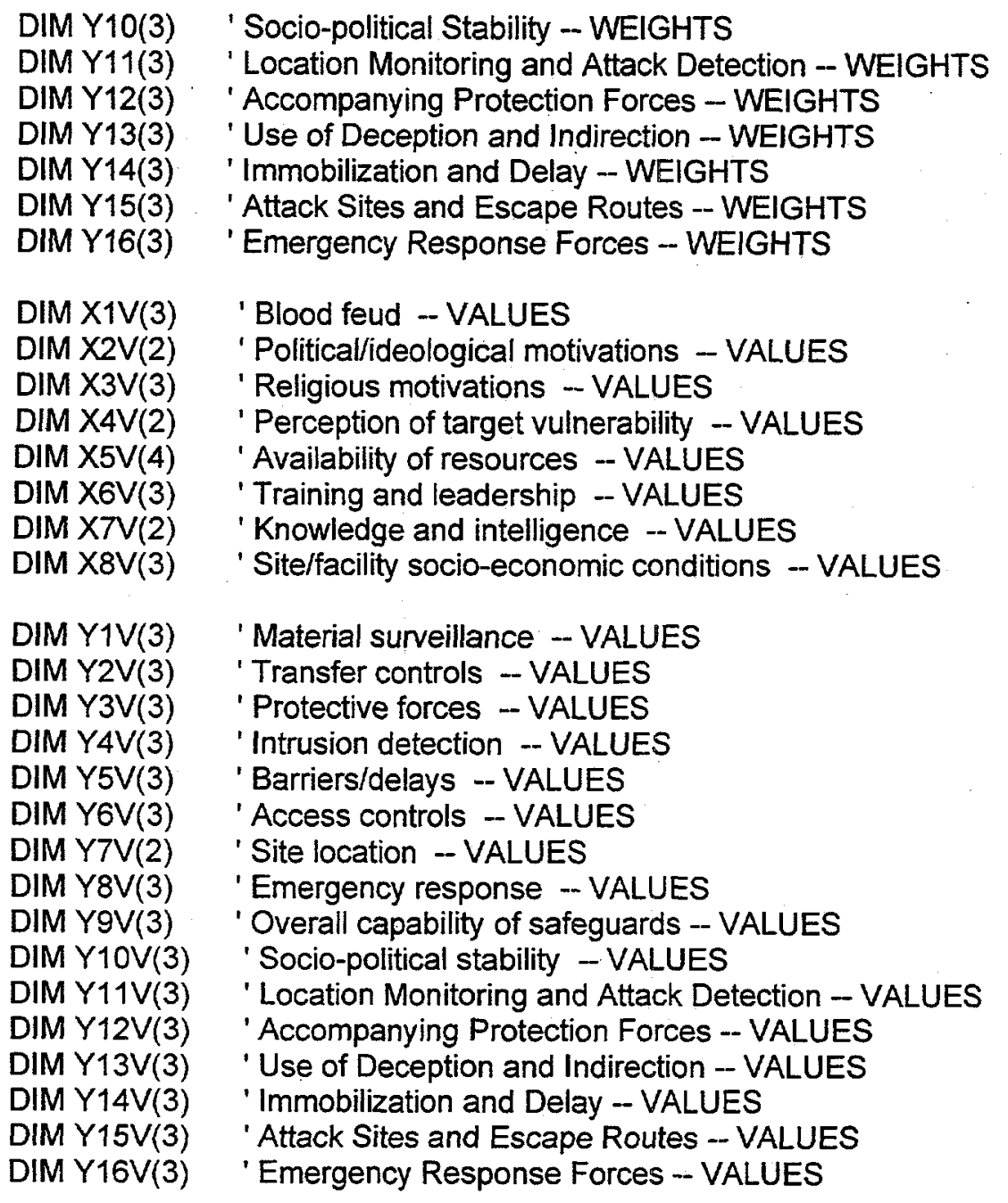

FOR I = 1 TO 3: READ X1V(I): NEXT I DATA 0.5, 1.0, 2.0

FOR I = 1 TO 2: READ X2V(I): NEXT I

DATA $0.5,1.0$

FOR I = 1 TO 3: READ X3V(I): NEXT I

DATA $0.5,1.0,2.0$

FOR I = 1 TO 2: READ X4V(I): NEXT I

DATA $0.5,1.5$

FOR I = 1 TO 4: READ X5V(I): NEXT I

DATA $0.0,0.5,1.0,2.0$

FOR I = 1 TO 3: READ X6V(1): NEXT I

DATA $0.5,1.0,2.0$

FOR I = 1 TO 2: READ X7V(I): NEXT I

DATA $0.5,1.5$

FOR I = 1 TO 3: READ X8V(I): NEXT I

DATA $0.75,1.0,1.5$

FOR I = 1 TO 3: READ Y1V(I): NEXT I

DATA $0.5,1.0,2.0$ 
FOR I = 1 TO 3: READ Y2V(I): NEXT I

DATA $0.5,1.0,2.0$

FOR I = 1 TO 3: READ Y3V(I): NEXT I

DATA 0.5, 1.0, 2.0

FOR I = 1 TO 3: READ Y4V(I): NEXT I

DATA $0.5,1.0,2.0$

FOR I = 1 TO 3: READ Y5V(I): NEXT I

DATA $0.5,1.0,2.0$

FOR I = 1 TO 3: READ YGV(I): NEXT I

DATA $0.5,1.0,2.0$

FOR I = 1 TO 2: READ Y7V(I): NEXT I

DATA $0.5,1.5$

FOR I = 1 TO 3: READ Y8V(I): NEXT I

DATA $0.5,1.0,2.0$

FOR I = 1 TO 3: READ Y YV(I): NEXT I

DATA $0.5,1.0,2.0$

FOR I = 1 TO $3:$ READ Y1OV(I): NEXT I

DATA 0.5, 1.0, 2.0

FOR I = 1 TO 3: READ Y $11 \mathrm{~V}(\mathrm{I})$ : NEXT I

DATA $0.5,1.0,2.0$

FOR I = 1 TO 3: READ Y12V(I): NEXT I

DATA $0.5,1.0,2.0$

FOR 1 = 1 TO 3 : READ Y $13 \mathrm{~V}(\mathrm{I})$ : NEXT I

DATA $0.75,1.0,1.5$

FOR I = 1 TO 3: READ Y $14 \mathrm{~V}(\mathrm{I})$ : NEXT I

DATA $0.5,1.0,1.25$

FOR I = 1 TO 3: READ Y15V(I): NEXT I

DATA $0.5,1.0,2.0$

FOR I = 1 TO 3: READ Y16V(I): NEXT I

DATA $0.5,1.0,2.0$

\section{DIM OATHREATPROB!(101)}

DIM OAVULNPROB!(101)

DIM THREAT(4) 'Threat category probabilities

DIM VULN(4) 'Vulnerability category probabilities

DIM LHOOD!(5) 'Likelihood category probabilities

DIM LMAT! $(4,4)$ 'Likelihood matrix probabilities

DIM CONSEQ(5) 'Consequence category probabilities

DIM RISK $(5,5)$ 'Risk matrix probabilities

DIM RISKCAT(5) 'Risk category probabilities

DIM scentype\$(5) ' Scenario type names

FOR I = 1 TO 5: READ scentype\$(I): NEXT I

DATA "Insider theft", "Outsider theft", "Outsider attack", "State transfer"

DATA "Diversion during Transportation"

' Define printout formats

$\mathrm{a} 2 \$=$ "\&\#.\#\# \&\#.\#"

D.73 
a3\$ = "\&\#.\#\# \& \#.\#\# \&\#.\#"

a4\$ = "\&\#.\#\# \& \#.\#\# \& \#.\#\# \&\#.\#\#"

tV $\$=$ "\& \#\#.\#\# \&\#.\#".

$\mathrm{C} \$=" \& \#$ \#\#"

IC\$ = "\&\# \&\#.\#"

INPUT "Enter name of output file "; outfile\$

OPEN outfile\$ FOR OUTPUT AS \#1

INPUT "Enter site/facility name "; sitename\$

PRINT \#1, "Evaluation of site/facility: "; sitename\$

PRINT \#1, "For scenario type: . "; scentype\$(5)

PRINT \#1, "Output file name: $\quad$ "; outfile\$

PRINT \#1, "Program and version Version 4 of XP_EVAL"

PRINT \#1,

GOSUB 5000

STOP

' Subroutines 110 through 360 solicit evaluator input for Threat and

' Vulnerability sub-measures. Subroutine 100 explains that the Threat

' and Vulnerability sub-measures are all treated as random variables,

' requiring that probabilities (or weights) summing to 1 must be assigned

' to each of the numerical values taken on by that sub-measure.

100 CLS

PRINT "The Threat and Vulnerability sub-measures used in this scheme"

PRINT "for estimating the risk of diversion of nuclear materials are"

PRINT "all treated as random variables, requiring that probabilities"

PRINT "(or weights) summing to 1 must be assigned to each of the"

PRINT "numerical values taken on by that sub-measure. The input routines"

PRINT "will enforce this requirement."

PRINT "'!'

PRINT "In addition, the assignment of probabilities to the sub-measure"

PRINT "can be used to represent the evaluators uncertainty about the"

PRINT "correct value of the sub-measure. For instance, in the absence of"

PRINT "any information about the true value of the sub-measure"

PRINT "(i.e., complete uncertainty), the evaluator should assign equal"

PRINT "weights to all of the sub-measure values."

PRINT "'!'

PRINT "Touch any key to continue"

DO

LOOP UNTIL INKEY\$ <> "'"

RETURN

110 CLS

PRINT "Existence of a Long Standing Conflict "

PRINT "'! 
PRINT " X_1 $=0.5$, no underlying conflict"

PRINT " $\quad X-1=1.0$, recently developed conflict (decades-long duration)"

PRINT " $X \_1=2.0$, long standing conflict (centuries-long duration)"

PRINT "'"

PRINT "*Long Standing Conflict* is shorthand for aspects of intensity "

PRINT "and long duration in the motivation of the threat groups. It can "

PRINT "derive from extreme religious prejudice, language/cultural "

PRINT "differences, and/or from a long history of oppression,"

PRINT "suppression, or warfare between two groups of people. Examples"

PRINT "include Catholic Irish vs. Irish Protestants and the British "

PRINT "or the Basques vs. the Spanish or Islamic fundamentals vs."

PRINT "secular Moslem governments. "

PRINT "'

INPUT "Input three X_1 weights "; X1(1), X1(2), X1(3)

IF ABS $(X 1(1)+X 1(2)+X 1(3)-1 !)>$ EPS THEN

PRINT "Your weights for $X$ 1 should add up to 1.0."

PRINT "Please repeat the weight assignment."

PRINT "Touch any key to continue"

DO

LOOP UNTIL INKEY\$ $>>$ '"'

GOTO 110

END IF

PRINT \#1, "Variable X1 is Existence of a Long Standing Conflict"

PRINT \#1, USING a3\$; "X_1 values are "; X1V(1); " "; X1V(2); " "; X1V(3)

PRINT \#1, USING a3\$; "X_1 weights are "; X1(1); " "; X1(2); " "; X1(3)

PRINT \#1,

RETURN

120 CLS

PRINT "Political/ldeological Motivations "

PRINT "'

PRINT " X_2 $=0.5$, no political/ideological basis "

PRINT " X_2 $=1.0$, political/ideological motivations"

PRINT "'!

PRINT "Examples would be support for terrorism as a component "

PRINT "of the cold war struggle between Communism and the West."

PRINT "'!

INPUT "Input two X_2 weights "; X2(1), X2(2)

IF ABS $(X 2(1)+X 2(\overline{2})-1$ !) $>$ EPS THEN

PRINT "Your weights for X_2 should add up to 1.0."

PRINT "Please repeat the weight assignment."

PRINT "Touch any key to continue"

DO

LOOP UNTIL INKEY\$ <> "'"

GOTO 120

END IF

PRINT \#1, "Variable X2 is Political//deological Motivations"

PRINT \#1, USING a2\$; "X_2 values are "; X2V(1); " "; X2V(2)

PRINT \#1, USING a2\$; "X_2 weights are "; X2(1); " "; X2(2)

PRINT \#1, 


\section{RETURN}

130 CLS

PRINT "Religious Motivations "

PRINT "

PRINT " X_3 $=0.5$, no religious animosity"

PRINT " $\quad X \_3=1.0$, some religious animosity"

PRINT " $\quad X \_3=2.0$, strong religious animosity"

PRINT "'!

PRINT "The intent here is to capture aspects of motivation distinct "

PRINT "from possible religious components of the "; blood; feud; " measure "

PRINT "above."

PRINT "'!

INPUT "Input three $X \_3$ weights "; $X 3(1), X 3(2), X 3(3)$

IF ABS $(X 3(1)+X 3(2)+X 3(3)-1$ !) $>$ EPS THEN

PRINT "Your weights for $X \_3$ should add up to 1.0."

PRINT "Please repeat the weight assignment."

PRINT "Touch any key to continue"

DO

LOOP UNTIL INKEY\$ $<>$ '"'

END IF GOTO 130

PRINT \#1, "Variable X3 is Religious Motivations"

PRINT \#1, USING a3\$; "X_3 values are "; X3V(1); " "; X3V(2); " "; X3V(3)

PRINT \#1, USING a3\$; "X_3 weights are "; X3(1);" "; X3(2); " "; X3(3)

PRINT \#1,

\section{RETURN}

140 CLS

PRINT "Perception of target vulnerability "

PRINT "'"

PRINT " X_4 $=0.5$, facility not perceived as vulnerable "

PRINT " X_4 $=1.5$, facility perceived as vulnerable"

PRINT '"'

PRINT "The reasoning here is that, even for a threat group predisposed "

PRINT "to action, if a particular facility is perceived as not vulnerable, "

PRINT "they will direct their attention elsewhere."

PRINT "'!

INPUT "Input two $X \_4$ weights "; $X 4(1), X 4(2)$

IF ABS $(X 4(1)+X 4(\overline{2})-1$ !) $>$ EPS THEN

PRINT "Your weights for $X \_4$ should add up to 1.0."

PRINT "Please repeat the weight assignment."

PRINT "Touch any key to continue"

DO

LOOP UNTIL INKEY\$ <> "'

GOTO 140

END IF

PRINT \#1, "Variable X4 is Perception of Target Vulnerability"

PRINT \#1, USING a2\$; "X_4 values are "; X4V(1); " "; X4V(2)

PRINT \#1, USING a2\$; "X_4 weights are "; $X 4(1)$; " "; X4(2)

PRINT \#1, 


\section{RETURN}

\section{CLS}

PRINT "Availability of resources "

PRINT "'"

PRINT " X_5 $=0.0$, no resources available"

PRINT " X_5 $=0.5$, modest, locally collected resources"

PRINT " X_5 $=1.0$, intermediate, state-supported"

PRINT " X_5 = 2.0, large, state-supported"

PRINT "'t

PRINT "This is a measure of the resource base of the threat group."

PRINT "'!

INPUT "Input four X_5 weights "; $X 5(1), X 5(2), X 5(3), X 5(4)$

IF ABS $(X 5(1)+X 5(2)+X 5(3)+X 5(4)-1$ !) $>$ EPS THEN

PRINT "Your weights for $X \_5$ should add up to $1.0 . "$

PRINT "Please repeat the weight assignment."

PRINT "Touch any key to continue"

DO

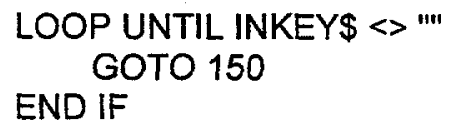




\section{RETURN}

170 CLS

PRINT "Knowledge and intelligence "

PRINT '"'

PRINT " X_7 $=0.5$, poor intelligence capabilities"

PRINT " $X \_7=1.5$, good intelligence capabilities"

PRINT "'!

PRINT "This is a measure of the access of the threat organization "

PRINT "to information about the potential target and their ability "

PRINT "to use that information."

PRINT "'!

INPUT "Input two $X \quad 7$ weights "; $X 7(1), X 7(2)$

IF ABS(X7(1) + X7(2) - 1!) > EPS THEN

PRINT "Your weights for $X \_7$ should add up to 1.0."

PRINT "Please repeat the weight assignment."

PRINT "Touch any key to continue"

DO

LOOP UNTIL INKEY\$ <> "'"

GOTO 170

END IF

PRINT \#1, "Variable $X 7$ is Threat Group Knowledge and Intelligence Capability"

PRINT \#1, USING a2\$; "X_7 values are "; X7V(1); " "; X7V(2)

PRINT \#1, USING a2\$; "X_7 weights are "; $X 7(1) ; "$ "; $X 7(2)$

PRINT \#1,

\section{RETURN}

$180 \mathrm{CLS}$

PRINT "Site/facility socio-economic conditions "

PRINT "'"

PRINT " X_8 $=0.75$, good conditions"

PRINT" $\quad X \_8=1.0$, not-so-good"

PRINT " $\quad X \_8=1.5$, extremely difficult conditions"

PRINT 'm'

PRINT "This attempts to measure the likelihood that the threat "

PRINT "organization can subvert/coopt insiders to support its "

PRINT "diversion attempt or that insiders will themselves attempt diversion."

PRINT '"'

INPUT "Input three $X \_8$ weights "; $X 8(1), X 8(2), X 8(3)$

IF ABS(X8(1) +X8(2) + X8(3) - 1!) > EPS THEN

PRINT "Your weights for $X \_8$ should add up to 1.0."

PRINT "Please repeat the weight assignment."

PRINT "Touch any key to continue"

DO

LOOP UNTIL INKEY\$ <> "'"

GOTO 180

END IF

PRINT \#1, "Variable X8 is Site/Facility Socio-Economic Conditions"

PRINT\#1, USING a3\$; "X_8 values are "; X8V(1); " "; X8V(2); " "; X8V(3)

PRINT \#1, USING a3\$; "X_8 weights are "; X8(1); " "; $X 8(2)$; " "; $X 8(3)$ 
PRINT \#1,

RETURN

210 CLS

PRINT "Material Surveillance "

PRINT "'

PRINT " $\quad Y_{-} 1=0.5$, excellent system of material surveillance"

PRINT " $Y_{-}^{-1}=1.0$, good system of material surveillance"

PRINT " $Y_{-}^{-1}=2.0$, poor system of material surveillance"

PRINT "'"

PRINT "This measures the material accounting and surveillance "

PRINT "infrastructure that enables facility management to detect "

PRINT "attempted or successful diversion."

PRINT "'!

INPUT "Input three $Y \_1$ weights "; $Y 1(1), Y 1(2), Y 1(3)$

IF ABS(Y1(1) + Y1(2) + Y1(3) - 1!) > EPS THEN

PRINT "Your weights for $Y_{-} 1$ should add up to 1.0."

PRINT "Please repeat the weight assignment."

PRINT "Touch any key to continue"

DO

LOOP UNTIL INKEY\$ $<>$

GOTO 210

END IF

PRINT \#1, "Variable $Y 1$ is Site Material Surveillance"

PRINT \#1, USING a3\$; "Y_1 values are "; Y1V(1); " "; Y1V(2); " "; Y1V(3)

PRINT \#1, USING a3\$; "Y_1 weights are "; $Y 1(1) ; "$ " "; $Y 1(2) ;$ " "; $Y 1(3)$

PRINT \#1,

\section{RETURN}

220 CLS

PRINT "Transfer Controls "

PRINT "'

PRINT " $\quad Y_{-} 2=0.5$, excellent system of transfer control"

PRINT " $\quad Y_{2}=1.0$, good system of transfer control"

PRINT " $Y_{-} 2=2.0$, poor system of transfer control"

PRINT "'m

PRINT "This measures the security of material in transit from one "

PRINT "storage/utilization location to another."

PRINT "'!

INPUT "Input three Y_2 weights "; Y2(1), Y2(2), Y2(3)

IF ABS(Y2(1) + Y2(2) + Y2(3) - 1!) > EPS THEN

PRINT "Your weights for $Y \_2$ should add up to 1.0."

PRINT "Please repeat the weight assignment."

PRINT "Touch any key to continue"

DO

LOOP UNTIL INKEY\$ <> "'

GOTO 220

END IF

PRINT \#1, "Variable Y2 is Site Transfer Controls" 
PRINT \#1, USING a3\$; "Y_2 values are "; Y2V(1); " "; Y2V(2); " "; Y2V(3)

PRINT \#1, USING a3\$; "Y_2 weights are "; Y2(1); " "; Y2(2); " "; Y2(3)

PRINT \#1,

\section{RETURN}

230 CLS

PRINT "Protective Forces "

PRINT "'!

PRINT" $Y \_3=0.5$, excellent protective forces"

PRINT " $Y^{-} 3=1.0$, good protective forces"

PRINT " Y $3=2.0$, poor protective forces"

PRINT "'!

PRINT "This is a measure of the ability of the facility guard forces "

PRINT "to prevent or delay a diversion."

PRINT "'

INPUT "Input three $Y \_3$ weights "; $Y 3(1), Y 3(2), Y 3(3)$

IF ABS(Y3(1) + Y3(2) + Y3(3) - 1!) > EPS THEN

PRINT "Your weights for $Y_{3} 3$ should add up to 1.0."

PRINT "Please repeat the weight assignment."

PRINT "Touch any key to continue"

DO

LOOP UNTIL INKEY\$ $<>$

GOTO 230

END IF

PRINT \#1, "Variable Y 3 is Site Protective Forces"

PRINT \#1, USING a3\$; "Y_3 values are "; Y3V(1); " "; Y3V(2); " "; Y3V(3)

PRINT \#1, USING a3\$; "Y_3 weights are "; Y3(1); " "; Y3(2); " "; Y3(3)

PRINT \#1,

\section{RETURN}

240 CLS

PRINT "Intrusion Detection "

PRINT '"'

PRINT " Y $4=0.5$, excellent intrusion detection"

PRINT " $Y^{-4} 4=1.0$, good intrusion detection"

PRINT " Y $4=2.0$, poor intrusion detection"

PRINT "'"

PRINT "This measures infrastructure provisions for the detection of "

PRINT "outsider attack or theft attempts."

PRINT "'"

INPUT "Input three Y_4 weights "; Y4(1), Y4(2), Y4(3)

IF ABS(Y4(1)+Y4(2)+Y4(3) - 1!) > EPS THEN

PRINT "Your weights for $Y_{4} 4$ should add up to 1.0."

PRINT "Please repeat the weight assignment."

PRINT "Touch any key to continue"

DO

LOOP UNTIL INKEY\$ <> "'"

GOTO 240

END IF

PRINT \#1, "Variable Y4 is Site Intrusion Detection" 
PRINT \#1, USING a3\$; "Y_4 values are "; Y4V(1); "; Y4V(2); " "; Y4V(3)

PRINT \#1, USING a3\$; "Y_4 weights are "; Y4(1); " "; Y4(2); " "; Y4(3)

PRINT \#1,

\section{RETURN}

250 CLS

PRINT "Barriers/Delays "

PRINT "'"'

PRINT " Y_5 $=0.5$, excellent barriers/delays"

PRINT " Y_5 = 1.0, good barriers/delays"

PRINT " $Y_{-}^{-5}=2.0$, poor barriers/delays"

PRINT "'"

PRINT "This measures facility design and operation features that "

PRINT "tend to delay a diversion attempt long enough for response "

PRINT "of off-site protection forces."

PRINT "'!

INPUT "Input three $Y \_5$ weights "; $Y 5(1), Y 5(2), Y 5(3)$

IF ABS(Y5(1) + Y5(2) + Y5(3) - 1!) > EPS THEN

PRINT "Your weights for $Y \_5$ should add up to 1.0."

PRINT "Please repeat the weight assignment."

PRINT "Touch any key to continue"

DO

LOOP UNTIL INKEY\$ <> "'

GOTO 250

END IF

PRINT \#1, "Variable Y5 is Site Barriers/Delays"

PRINT \#1, USING a3\$; "Y_5 values are "; Y5V(1); " "; Y5V(2); " "; Y5V(3)

PRINT \#1, USING a3\$; "Y_5 weights are "; Y5(1); " "; Y5(2); " "; Y5(3)

PRINT \#1,

\section{RETURN}

260 CLS

PRINT "Access Controls "

PRINT "m!

PRINT " $\quad Y_{-} 6=0.5$, excellent access controls"

PRINT " $Y 6=1.0$, good access controls"

PRINT " Y_6 $6=2.0$, poor access controls"

PRINT '"'

PRINT "This measures controls aimed at preventing outsider access "

PRINT "to material storage areas and to constraining the ability "

PRINT "of insiders to gradually divert materials."

PRINT "'!

INPUT "Input three $Y_{-} 6$ weights "; $Y 6(1), Y 6(2), Y 6(3)$

IF ABS(Y6(1) + Y6(2) + Y6(3) - 1!) > EPS THEN

PRINT "Your weights for $Y_{-} 6$ should add up to 1.0."

PRINT "Please repeat the weight assignment."

PRINT "Touch any key to continue"

DO

LOOP UNTIL INKEY\$ <> "'"

GOTO 260 
END IF

PRINT \#1, "Variable Y6 is Site Access Controls"

PRINT \#1, USING a3\$; "Y_6 values are "; Y6V(1); " "; Y6V(2); " "; Y6V(3)

PRINT \#1, USING a3\$; "Y_6 weights are "; Y6(1); " "; Y6(2); " "; Y6(3)

PRINT \#1,

RETURN

270 CLS

PRINT "Site Location "

PRINT "'"

PRINT " Y $7=0.5$, site with none or few good escape routes"

PRINT " $Y_{-} 7=1.5$, site with many good escape paths"

PRINT "'

PRINT "This measures the ability of group or individual that "

PRINT "has successfully diverted material to successfully remove "

PRINT "it to a secure location and/or transfer it to the end-user. "

PRINT "'!

INPUT "Input two $Y$ 7 weights "; $Y 7(1), Y 7(2)$

IF ABS $(Y 7(1)+Y 7(\overline{2})-1$ !) $>$ EPS THEN

PRINT "Your weights for $Y_{-} 7$ should add up to 1.0."

PRINT "Please repeat the weight assignment."

PRINT "Touch any key to continue"

DO

LOOP UNTIL INKEY\$ <> "'

GOTO 270

END IF

PRINT \#1, "Variable Y7 is Site Location (as it affects interdiction of "

PRINT \#1, " diverted materials)"

PRINT \#1, USING a2\$; "Y_7 values are "; Y7V(1); " "; Y7V(2)

PRINT \#1, USING a2\$; "Y_7 weights are "; Y7(1); " "; Y7(2)

PRINT \#1,

\section{RETURN}

\section{CLS}

PRINT "Emergency Response "

PRINT "m

PRINT " $\quad Y_{-} 8=0.5$, site with excellent emergency response resources"

PRINT " $Y$ - $8=1.0$, site with some emergency response"

PRINT " Y $Y_{-}^{-8}=2.0$, site with none or poor emergency response"

PRINT '"'

PRINT "This measures the ability of off-site emergency response to "

PRINT "interdict a forcible diversion in progress, given timely "

PRINT "notification. Note that measures $Y_{-} 4, Y_{-} 5$, and $Y_{-} 7$ also "

PRINT "condition the likelihood of successful interdiction of a "

PRINT "diversion in progress. "

PRINT "'!'

INPUT "Input three $Y_{-} 8$ weights "; $Y 8(1), Y 8(2), Y 8(3)$

IF ABS(Y8(1) + Y8(2) + Y8(3) - 1!) > EPS THEN

PRINT "Your weights for $Y_{-} 8$ should add up to 1.0."

PRINT "Please repeat the weight assignment." 
PRINT "Touch any key to continue"

DO

LOOP UNTIL INKEY\$ <> "' GOTO 280

END IF

PRINT \#1, "Variable Y8 is Site Emergency Response (as it affects "

PRINT \#1, " interdiction of diverted materials)"

PRINT \#1, USING a3\$; "Y_8 values are "; Y8V(1); " "; Y8V(2); " "; Y8V(3)

PRINT \#1, USING a3\$; "Y_8 weights are "; Y8(1); " "; Y8(2); " "; $Y 8(3)$

PRINT \#1,

\section{RETURN}

285 CLS

PRINT "Overall Capability of Host Country Safeguards Measures"

PRINT "'"

PRINT " Y $9=0.5$, Robust safeguards measures "

PRINT" Y_9 =1.0, Safeguards with some weaknesses in design "

PRINT " $\quad$ or implementation "

PRINT " Y_9 $=2.0$, Weak or non-existent safeguards "

PRINT "'+"

PRINT "This measures the overall capability of safeguards measures "

PRINT "to detect/alarm attempted state transfer of weapons material "

PRINT "to unauthorized state- or sub-state-organizations."

PRINT "'!

INPUT "Input three $Y$ _9 weights "; $Y 9(1), Y 9(2), Y 9(3)$

IF ABS(Y9(1) + Y9(2) + Y9(3) - 1!) > EPS THEN

PRINT "Your weights for $Y_{-} 9$ should add up to 1.0."

PRINT "Please repeat the weight assignment."

PRINT "Touch any key to continue"

DO

LOOP UNTIL INKEY\$ <> "'"

GOTO 285

END IF

PRINT \#1, "Variable Y9 is Overall Capability of Host Country Safeguards"

PRINT \#1, USING a3\$; "Y_9 values are "; Y9V(1); " "; Y9V(2); " "; Y9V(3)

PRINT \#1, USING a3\$; "Y_9 weights are "; Y9(1); " "; Y9(2); " "; Y9(3)

PRINT \#1,

\section{RETURN}

290 CLS

PRINT "Socio-political stability "

PRINT "'m

PRINT " Y_10 $=0.5$, stable government with good foreign "

PRINT" $\quad$ relations, checks and balances"

PRINT " Y_10 = 1.0, some instability, some foreign enemies, "

PRINT " $\quad$ some checks and balances"

PRINT " $Y_{-} 10=2.0$, significant instability, many factions, "

PRINT " - many enemies, no checks and balances"

PRINT "'!

PRINT "This attempts to measure the likelihood that all or part of " 
PRINT "the relevant government organizations will formulate and " PRINT "implement or will fail to impede a state transfer diversion "

PRINT "attempt. "

PRINT "':

INPUT "Input three Y_10 weights "; Y10(1), Y10(2), Y10(3)

IF ABS $(Y 10(1)+Y 10(2)+Y 10(3)-1$ !) $>$ EPS THEN

PRINT "Your weights for $Y_{-} 10$ should add up to 1.0."

PRINT "Please repeat the weight assignment."

PRINT "Touch any key to continue"

DO

LOOP UNTIL INKEY\$ <> "'!

GOTO 290

END IF

PRINT \#1, "Variable Y10 is Host Country Socio-political Stability"

PRINT \#1, USING a3\$; "Y_10 values are "; Y10V(1); " "; Y10V(2); " "; Y10V(3)

PRINT \#1, USING a3\$; "Y_10 weights are "; Y10(1); " "; Y10(2); " "; Y10(3)

PRINT \#1,

\section{RETURN}

$310 \mathrm{CLS}$

PRINT "Location Monitoring and Attack Detection"

PRINT '"'

PRINT " $Y_{-} 11=0.5$, excellent location monitoring and attack detection"

PRINT " $Y^{-11}=1.0$, good location monitoring and attack detection"

PRINT " $Y_{-}^{-11}=2.0$, poor location monitoring and attack detection"

PRINT "'"

PRINT "This measures the existence or lack of real-time systems for "

PRINT "monitoring the location of the shipment and detecting an attack"

PRINT "on the shipment. This information is needed to enable emergency"

PRINT "response by protection forces other than those accompanying the "

PRINT "shipment."

PRINT '!"'

INPUT "Input three Y_11 weights "; Y11(1), Y11(2), Y11(3)

IF ABS $(Y 11(1)+Y 11(2)+Y 11(3)-1$ !) $>$ EPS THEN

PRINT "Your weights for $Y_{-} 11$ should add up to 1.0."

PRINT "Please repeat the weight assignment."

PRINT "Touch any key to continue"

DO

LOOP UNTIL INKEY\$ <> "'

GOTO 310

END IF

PRINT \#1, "Variable Y_11 measures Location Monitoring and Attack Detection"

PRINT \#1, USING a3\$; "Y_11 values are "; Y11V(1); " "; Y11V(2); " "; Y11V(3)

PRINT \#1, USING a3\$; "Y_11 weights are "; Y11(1); " "; Y11(2); " "; Y11(3)

PRINT \#1,

RETURN

320 CLS

PRINT "Accompanying Protection Forces"

PRINT '"' 
PRINT " $\quad Y_{-} 12=0.5$, adequate protection forces accompanying shipment"

PRINT " $Y_{-}^{-12}=1.0$, some accompanying forces"

PRINT " $Y_{-}^{-12}=2.0$, no significant accompanying forces"

PRINT "'"

PRINT "This measures those protection forces accompanying the shipment and"

PRINT "available for immediate response to an attack on the shipment."

PRINT "'"

INPUT "Input three Y_12 weights "; Y12(1), Y12(2), Y12(3)

IF ABS $(Y 12(1)+Y 12(2)+Y 12(3)-1 !)>$ EPS THEN

PRINT "Your weights for $Y_{-} 12$ should add up to 1.0."

PRINT "Please repeat the weight assignment."

PRINT "Touch any key to continue"

DO

LOOP UNTIL INKEY\$ $<>$ "'"

END IF

GOTO 320

PRINT \#1, "Variable $Y$ _12 measures Accompanying Protection Forces"

PRINT \#1, USING a3\$; "Y_12 values are "; Y12V(1); " "; Y12V(2); " "; Y12V(3)

PRINT \#1, USING a3\$; "Y_12 weights are "; Y12(1); " "; $Y 12(2) ; "$ "; $Y 12(3)$

PRINT \#1,

\section{RETURN}

330 CLS

PRINT "Use of Deception and Indirection"

PRINT '"'

PRINT " Y_13 $=0.75$, Well-executed deception and indirection"

PRINT " $Y_{-} 13=1.0$, Some use of deception and indirection"

PRINT " $Y_{-}^{-13}=1.5$, No use of deception and indirection"

PRINT '"'

PRINT "This measures the use of deception and indirection to disguise the"

PRINT "time, planned itinerary, and planned protective systems for the"

PRINT "shipment."

PRINT "'!

INPUT "Input three $Y$ 13 weights "; $Y 13(1), Y 13(2), Y 13(3)$

IF ABS $(Y 13(1)+Y 13(2)+Y 13(3)-1$ ! $)>$ EPS THEN

PRINT "Your weights for $Y_{-} 13$ should add up to 1.0."

PRINT "Please repeat the weight assignment."

PRINT "Touch any key to continue"

DO

LOOP UNTIL INKEY\$ <> "'"

GOTO 330

END IF

PRINT \#1, "Variable $Y \_13$ is Deception and Indirection "

PRINT \#1, USING a3\$; "Y_13 values are "; Y13V(1); " "; Y13V(2);" ";Y13V(3)

PRINT \#1, USING a3\$; "Y_13 weights are "; Y13(1); " "; Y13(2);" ";Y13(3)

PRINT \#1,

RETURN

340 CLS

PRINT "Immobilization and Delay" 


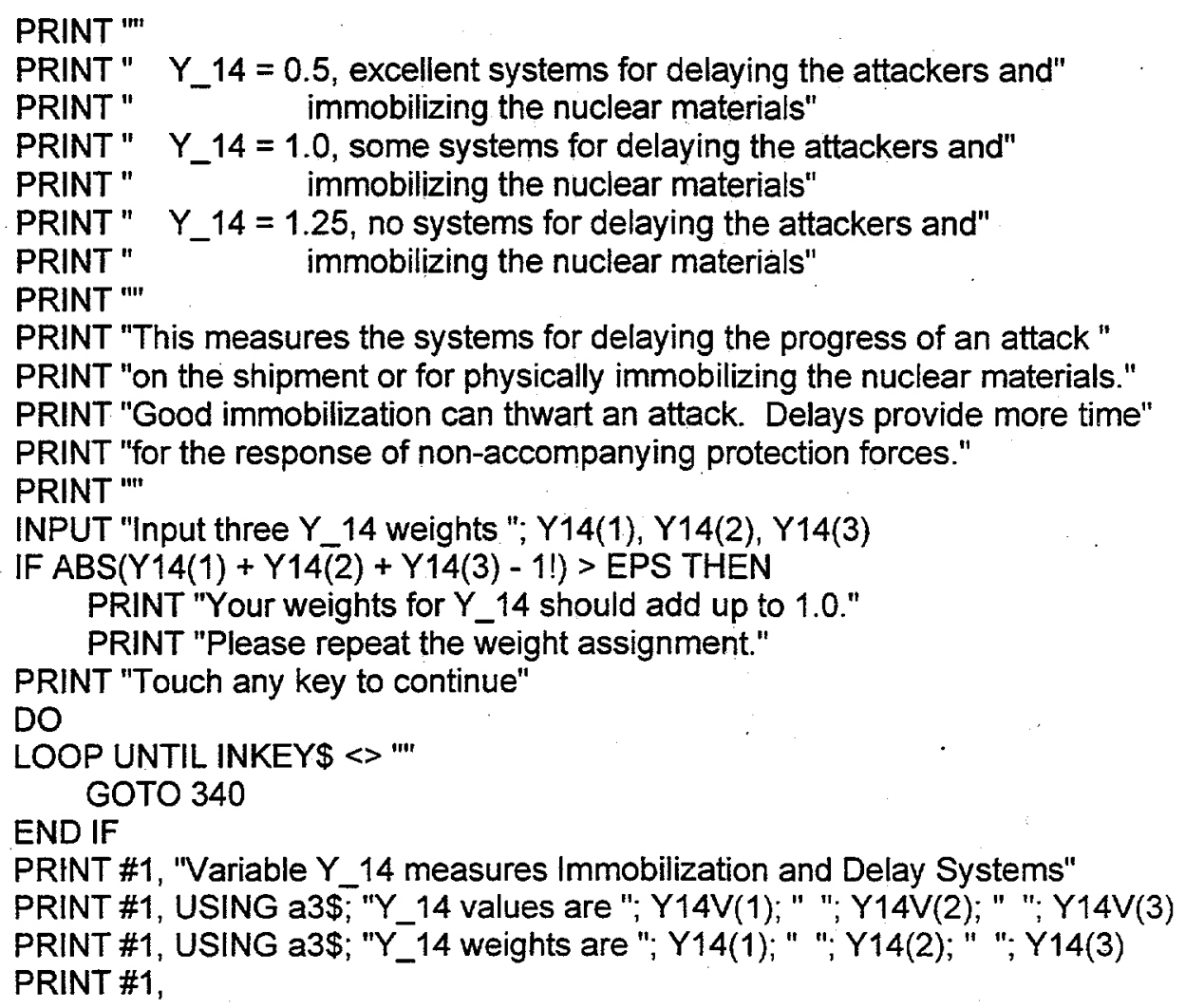

\section{RETURN}

350 CLS

PRINT "Attack Sites and Escape Routes"

PRINT "'"

PRINT " Y_15 $=0.5$, few attack sites and few escape routes"

PRINT " Y $Y_{-} 15=1.0$, intermediate mixture of attack sites and"

PRINT " $\quad$ escape routes"

PRINT " Y Y 15 = 2.0, many attack sites and many escape routes"

PRINT "'"

PRINT "This measures the ease with which a shipment can be attacked"

PRINT "and the variety of escape routes available after a successful"

PRINT "attack."

PRINT "'"

INPUT "Input three Y_15 weights "; Y15(1), Y15(2), Y15(3)

IF ABS $(Y 15(1)+Y 15 \overline{(2)}+Y 15(3)-1$ !) > EPS THEN

PRINT "Your weights for $Y_{-} 15$ should add up to 1.0."

PRINT "Please repeat the weight assignment."

PRINT "Touch any key to continue"

DO

LOOP UNTIL INKEY\$ <> "'"

END IF

GOTO 350

PRINT \#1, "Variable $Y \_15$ measures Attack Sites and Escape Routes"

PRINT \#1, USING a3\$; "Y_15 values are "; Y15V(1); " "; Y15V(2); " "; Y15V(3) 
PRINT \#1, USING a3\$; "Y_15 weights are "; Y15(1); " "; Y15(2); " "; Y15(3)

PRINT \#1,

\section{RETURN}

$360 \mathrm{CLS}$

PRINT "Non-accompanying Emergency Response Forces"

PRINT "'!

PRINT " Y_16 $=0.5$, excellent array of protection forces available to"

PRINT " $\quad$ respond to an attack in progress"

PRINT " $\quad Y_{-} 16=1.0$, some protection forces available to respond to"

PRINT " - respond to an attack in progress"

PRINT " Y_16 $=2.0$, no significant protection forces available to"

PRINT " - respond to an attack in progress"

PRINT "'"

PRINT "This measures the additional forces that can be brought to bear upon"

PRINT "an attack in progress or used to interdict the movement of diverted"

PRINT "material from the attack site to its intended customer."

PRINT "'”

INPUT "Input three Y_16 weights "; Y16(1), Y16(2), Y16(3)

IF ABS(Y16(1) + Y16(2) + Y16(3) - 1!) > EPS THEN

PRINT "Your weights for $Y_{-} 16$ should add up to 1.0."

PRINT "Please repeat the weight assignment."

PRINT "Touch any key to continue"

DO

LOOP UNTIL INKEY\$ <> "'"

GOTO 360

END IF

PRINT \#1, "Variable $Y \_16$ measures Non-accompanying Emergency Response Forces"

PRINT \#1, USING a3\$; "Y_16 values are "; Y16V(1); " "; Y16V(2); " "; Y16V(3)

PRINT \#1, USING a3\$; "Y_16 weights are "; Y16(1); " "; Y16(2); " "; Y16(3)

PRINT \#1,

\section{RETURN}

' Subroutine 5000 calculates Threat and Vulnerability Probabilities

' for the Outsider Attack scenario type

5000 GOSUB 100

GOSUB 110

GOSUB 120

GOSUB 140

GOSUB 150

GOSUB 160

GOSUB 170

GOSUB 180

GOSUB 310

GOSUB 320

GOSUB 330

GOSUB 340

GOSUB 350 


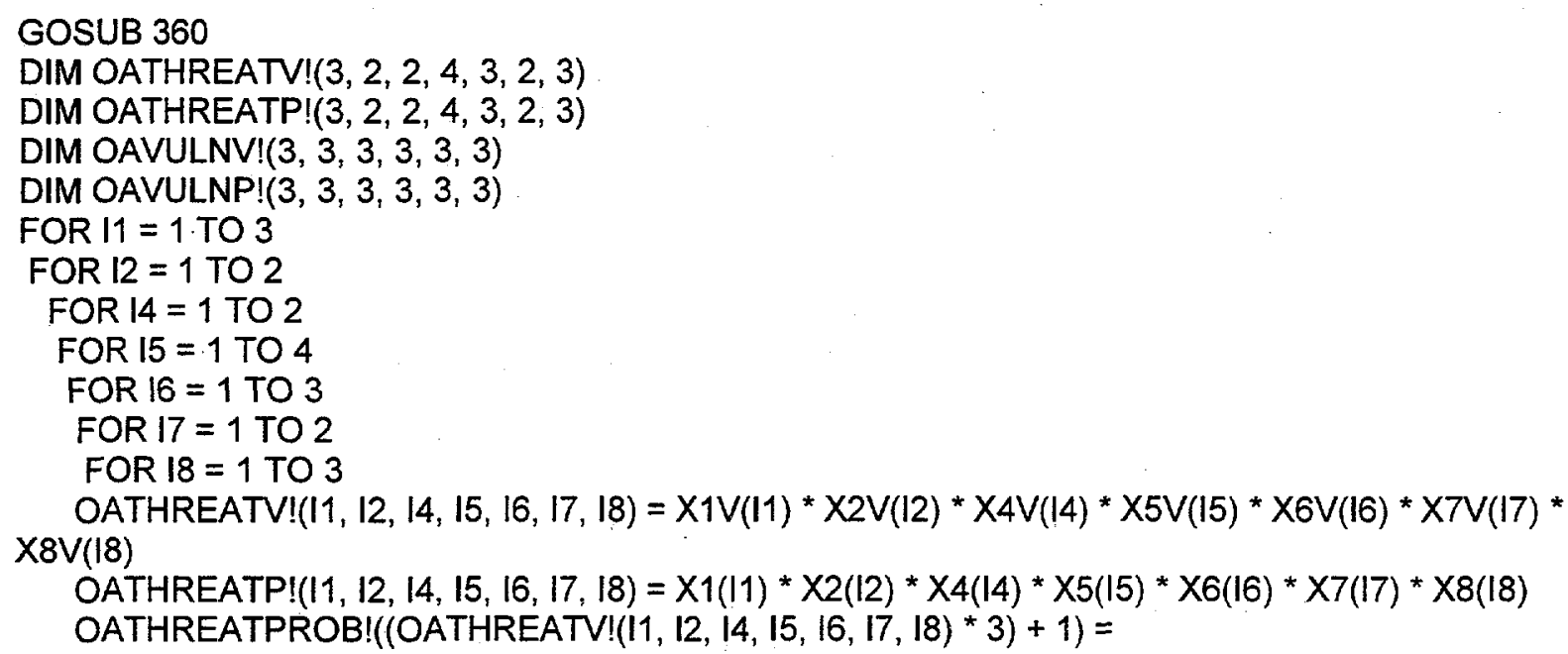


' NEXT I

PRINT \#1,

'FOR I = 1 TO 101

- PRINT \#1, USING tv\$; "For Vulnerability value = "; ( 1 - 1) / 3; " the probability is "; OAVULNPROB!(I)

' NEXTI

GOSUB 5050

GOSUB 5100

GOSUB 5200

\section{RETURN}

- Subroutine 5050 accumulates the Threat probabilities values into

' four Threat category probability values for the Outsider Attack

' scenario type; it also accumulates the Vulnerability probability

' values into four Vulnerability category probability values for the

' Outsider Attach scenario type.

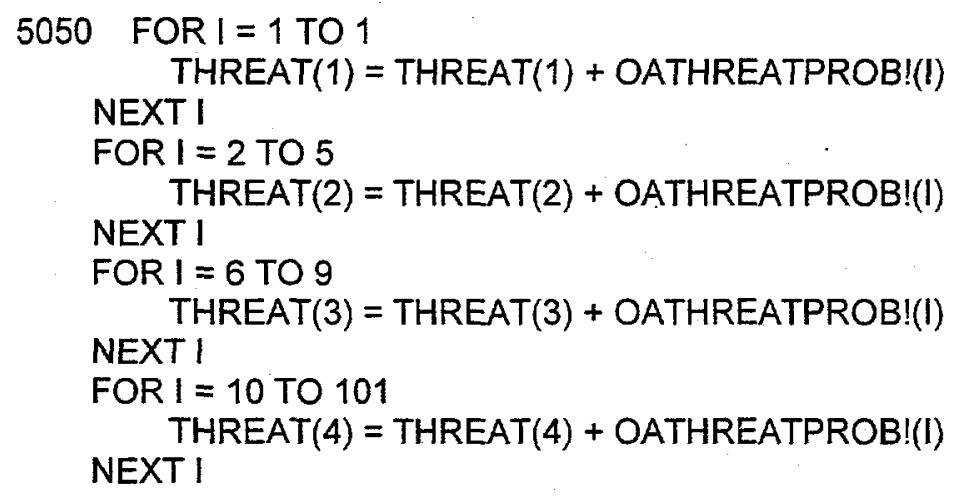

PRINT \#1,

PRINT \#1, USING c\$; "For Threat category Negligible, the probability is ";THREAT(1)

PRINT \#1, USING C\$; "For Threat category Low, the probability is ";THREAT(2)

PRINT \#1, USING C\$; "For Threat category High, the probability is ";THREAT(3)

PRINT \#1, USING C\$; "For Threat category Very High, the probability is ";THREAT(4)

PRINT \#1,

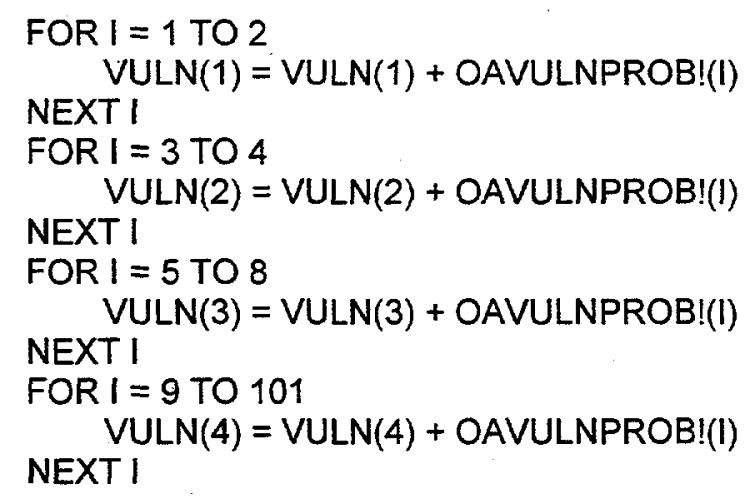


PRINT \#1,

PRINT \#1, USING c\$; "For Vulnerability category Negligible, the probability is ";VULN(1)

PRINT \#1, USING c\$; "For Vulnerability category Low, the probability is ";VULN(2)

PRINT \#1, USING C\$; "For Vulnerability category High, the probability is ";VULN(3)

PRINT \#1, USING c\$; "For Vulnerability category Very High, the probability is ";VULN(4)

PRINT \#1,

\section{RETURN}

- Subroutine 5100 computes the Likelihood matrix probability values and

' accumulates them into the five Likelihood category probabilities

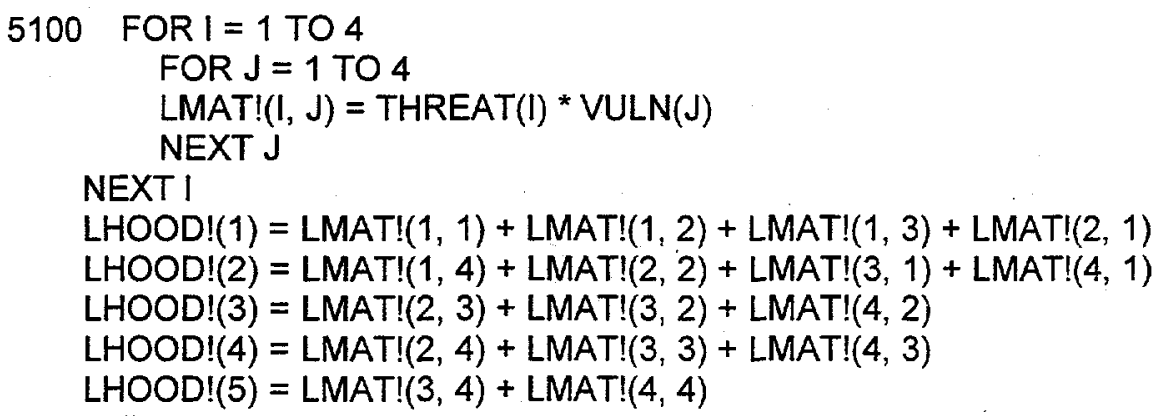

PRINT \#1,

PRINT \#1, USING c\$; "Probability for Likelihood category Negligible = "; LHOOD!(1)

PRINT \#1, USING c\$; "Probability for Likelihood category Low = "; LHOOD!(2)

PRINT \#1, USING c\$; "Probability for Likelihood category Medium = "; LHOOD!(3)

PRINT \#1, USING C\$; "Probability for Likelihood category High = "; LHOOD!(4)

PRINT \#1, USING C\$; "Probability for Likelihood category Very High = "; LHOOD!(5)

PRINT \#1,

\section{RETURN}

- Subroutine 5200 solicits input from the evaluator for the Consequence

- measure, given as weights/probabilities assigned to each of the five

- Consequence categories. This input is echoed to output. Finally,

- Risk Matrix probabilities are calculated and then accumulated into

' the five Risk category probabilities

\section{CLS}

PRINT "Enter estimate of site/facility diversion consequences."

PRINT "Enter five weights/probabilities associated with the categories:"

PRINT "'"

PRINT " Negligible = Fission products or other non-weapons material"

PRINT " (IAEA Attractiveness Level E or less)"

PRINT "'!

PRINT "

PRINT"

PRINT "

PRINT "

PRINT"

PRINT"

PRINT"

Low = Small amounts of difficult to convert materials"

(IAEA Attractiveness Level D, Category IV)"

Medium = Small amounts of easily convertible or large amounts" of difficult to convert materials"

(IAEA Attractiveness Level D, Categories I,II, or III" and Attractiveness Levels B and C, Category IV)" 


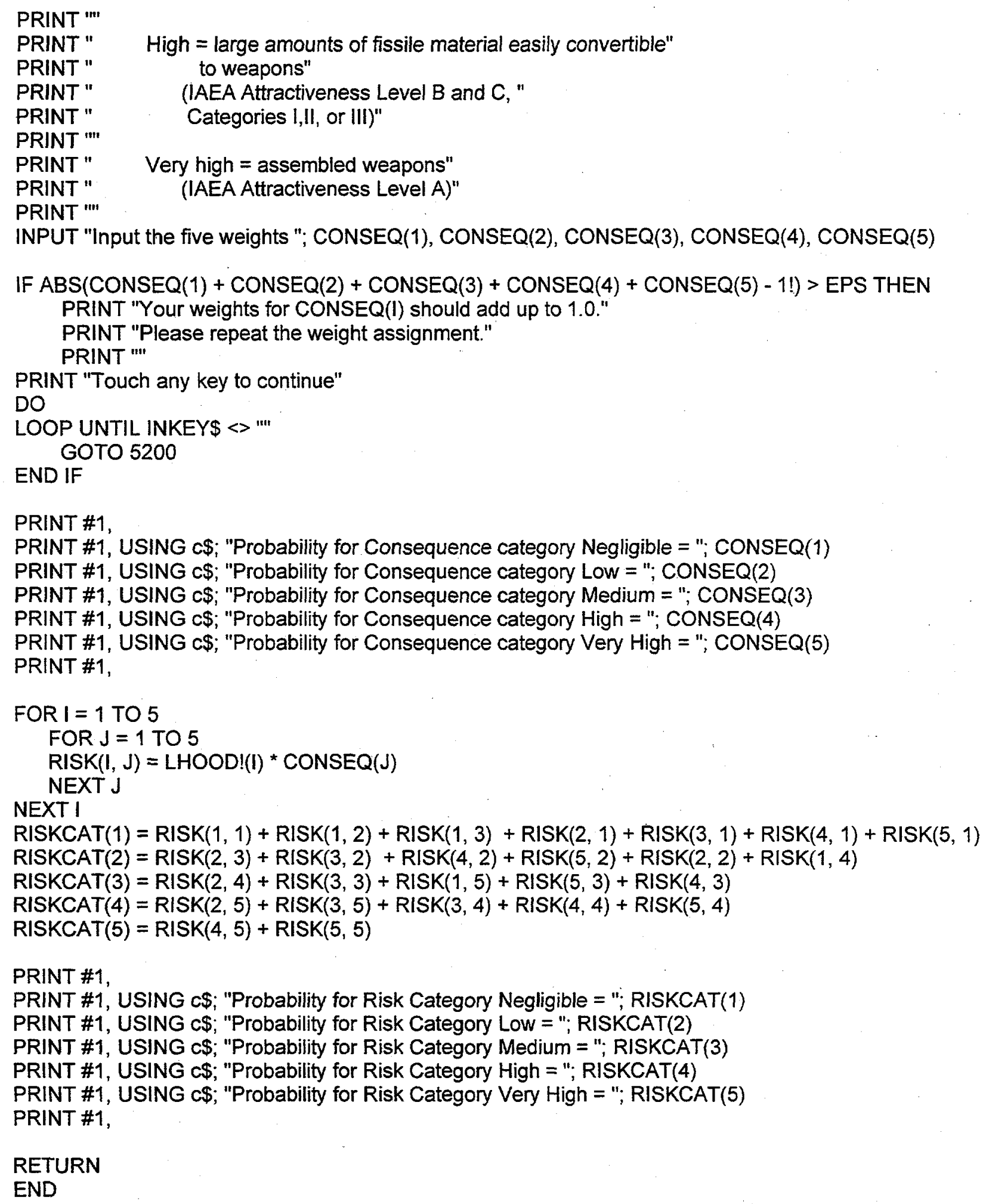




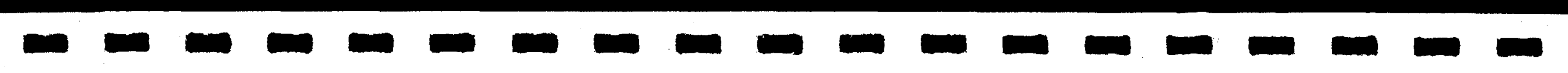

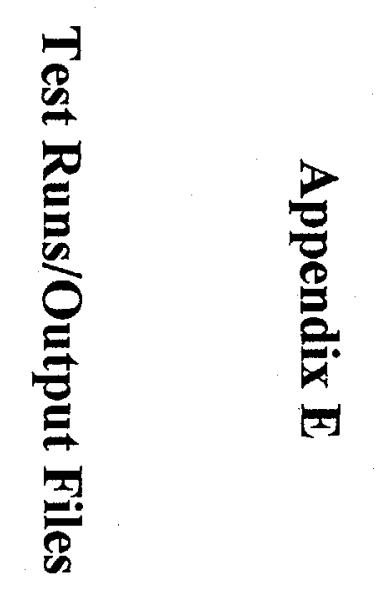




\section{APPENDIX E - Test Runs / Output Files}

\section{Test Output}

This Appendix contains 15 output files, three for each of the five generic diversion scenarios. Each output file reports the results of the test runs that were used to tune/calibrate the models. Each file has a header that gives the type of test input used and the scenario type, followed by an input echo, and finally the model output for that input.

Three types of test input were used: 1) a maximum uncertainty or non-informative input, 2) maximum threat and maximum vulnerability, and 3) minimum threat and minimum vulnerability.

Maximum uncertainty was represented by input probabilities equally split over the two, three, or four possible values of each of the threat and vulnerability sub-variables and the consequence variable.

Maximum threat and vulnerability assigned a probability of 0.7 to the largest of the possible values for each threat and vulnerability sub-variable and probability of 0.3 to the next largest.

Minimum threat and vulnerability did just the opposite.

Our expectation was that non-informative input should produce a roughly equal distribution of probabilities across the risk categories, that maximum input should produce risk category probabilities concentrated in the highest two categories, and that minimum input should produce risk category probabilities concentrated in the lowest two categories.

The programs were then tuned to produce the expected results.

E. 1 
Evaluation of site/facility: noinf

For scenario type: Insider theft

Output file name: noinf4it.ou4

Program and version: Version 4 of IT_EVAL

Variable $\mathrm{X} 4$ is Perception of Target Vulnerability

$X \_4$ values are $0.500 \quad 1.500$

$\mathrm{X}$ _4 weights are $0.500 \quad 0.500$

Variable $X 5$ is Availability of Threat Group Resources

$X \_5$ values are $0.500 \quad 1.500$

$X \_5$ weights are $0.500 \quad 0.500$

Variable $X 7$ is Threat Group Knowledge and Intelligence Capability

$X$. 7 values are $0.500 \quad 1.500$

$\mathrm{X} \_7$ weights are $0.500 \quad 0.500$

Variable $X 8$ is Site/Facility Socio-Economic Conditions

$X \_8$ values are $0.750 \quad 1.000 \quad 1.500$

$\begin{array}{llll}X \_8 \text { weights are } 0.333 & 0.333 & 0.334\end{array}$

Variable $\mathrm{Y} 1$ is Site Material Surveillance

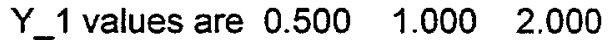

$\begin{array}{llll}Y_{-}{ }_{-} 1 \text { weights are } & 0.333 & 0.333 & 0.334\end{array}$

Variable $Y 2$ is Site Transfer Controls

Y_2 values are $0.500 \quad 1.000 \quad 2.000$

$\begin{array}{llll}Y_{-} \text {2 weights are } & 0.333 & 0.333 & 0.334\end{array}$

For Threat category Negligible, the probability is 0.125

For Threat category Low, the probability is 0.500

For Threat category High, the probability is 0.292

For Threat category Very High, the probability is 0.083

For Vulnerability category Negligible, the probability is 0.111

For Vulnerability category Low, the probability is 0.222

For Vulnerability category High, the probability is 0.333

For Vulnerability category Very High, the probability is 0.334

Probability for Likelihood category Negligible $=0.139$

Probability for Likelihood category Low $=0.194$

Probability for Likelihood category Medium $=0.250$

Probability for Likelihood category High $=0.292$

Probability for Likelihood category Very High $=0.125$

Probability for Consequence category Negligible $=0.200$

Probability for Consequence category Low $=0.200$ 
Probability for Consequence category Medium $=0.200$

Probability for Consequence category High $=0.200$

Probability for Consequence category Very High $=0.200$

Probability for Risk category Negligible $=0.255$

Probability for Risk category Low $=0.239$

Probability for Risk category Medium $=0.200$

Probability for Risk category High $=0.222$

Probability for Risk category Very High $=0.083$ 
Evaluation of site/facility: bigthreat_bigvuln_bigconseq_it

For scenario type:

Insider theft

Output file name:

it T\&V\&C.Ou 4

Program and version: Version 4 of IT_EVAL

Variable $\mathrm{X} 4$ is Perception of Target Vulnerability

$X \_4$ values are $0.500 \quad 1.500$

$\mathrm{X} \_4$ weights are $0.300 \quad 0.700$

Variable $X 5$ is Availability of Threat Group Resources

$X \quad 5$ values are $0.500 \quad 1.500$

$X \_5$ weights are $0.300 \quad 0.700$

Variable $\mathrm{X} 7$ is Threat Group Knowledge and Intelligence Capability

$X$. 7 values are $0.500 \quad 1.500$

$X \_7$ weights are $0.300 \quad 0.700$

Variable $\mathrm{X} 8$ is Site/Facility Socio-Economic Conditions

$X \_8$ values are $0.750 \quad 1.000 \quad 1.500$

$\begin{array}{llll}X \_8 \text { weights are } 0.000 & 0.300 & 0.700\end{array}$

Variable $Y 1$ is Site Material Surveillance

$Y$. 1 values are $0.500 \quad 1.000 \quad 2.000$

$\begin{array}{llll}Y_{-} 1 \text { 1 weights are } & 0.000 & 0.300 & 0.700\end{array}$

Variable $Y 2$ is Site Transfer Controls

$Y$ Y2 values are $0.500 \quad 1.000 \quad 2.000$

$\begin{array}{llll}Y \_2 \text { weights are } 0.000 & 0.300 & 0.700\end{array}$

For Threat category Negligible, the probability is 0.027

For Threat category Low, the probability is 0.189

For Threat category High, the probability is 0.441

For Threat category Very High, the probability is 0.343

For Vulnerability category Negligible, the probability is 0.000

For Vulnerability category Low, the probability is 0.000

For Vulnerability category High, the probability is 0.090

For Vulnerability category Very High, the probability is 0.910

Probability for Likelihood category Negligible $=0.002$

Probability for Likelihood category Low $=0.025$

Probability for Likelihood category Medium $=0.017$

Probability for Likelihood category High $=0.243$

Probability for Likelihood category Very High $=0.713$

Probability for Consequence category Negligible $=0.000$

Probability for Consequence category Low $=0.000$ 
Probability for Consequence category Medium $=0.300$

Probability for Consequence category High $=0.700$

Probability for Consequence category Very High $=0.000$

Probability for Risk category Negligible $=0.001$

Probability for Risk category Low $=0.009$

Probability for Risk category Medium $=0.309$

Probability for Risk category High $=0.681$

Probability for Risk category Very High $=0.000$ 
Evaluation of site/facility: smallthreat_smallvuln_smallconseq_it

For scenario type: Insider theft

Output file name: it-t-v-c.ou4

Program and version: Version 4 of IT_EVAL

Variable X4 is Perception of Target Vulnerability

$X 4$ values are $0.500 \quad 1.500$

$X$ _4 weights are $0.700 \quad 0.300$

Variable X5 is Availability of Threat Group Resources

$X$ _5 values are $0.500 \quad 1.500$

$X \_5$ weights are $0.700 \quad 0.300$

Variable $X 7$ is Threat Group Knowledge and Intelligence Capability

$X .7$ values are $0.500 \quad 1.500$

$X \_7$ weights are $0.700 \quad 0.300$

Variable X8 is Site/Facility Socio-Economic Conditions

$X \quad 8$ values are $0.750 \quad 1.000 \quad 1.500$

$\begin{array}{llll}X \text { _ } 8 \text { weights are } & 0.700 & 0.300 & 0.000\end{array}$

Variable $Y 1$ is Site Material Surveillance

$Y$ Y 1 values are $0.500 \quad 1.000 \quad 2.000$

$\begin{array}{llll}Y_{-}{ }_{-} 1 \text { weights are } & 0.700 & 0.300 & 0.000\end{array}$

Variable Y2 is Site Transfer Controls

$Y$ 2 values are $0.500 \quad 1.000 \quad 2.000$

$\begin{array}{llll}Y_{-} 2 \text { weights are } & 0.700 & 0.300 & 0.000\end{array}$

For Threat category Negligible, the probability is 0.343

For Threat category Low, the probability is 0.573

For Threat category High, the probability is 0.076

For Threat category Very High, the probability is 0.008

For Vulnerability category Negligible, the probability is 0.490

For Vulnerability category Low, the probability is 0.420

For Vulnerability category High, the probability is 0.090

For Vuinerability category Very High, the probability is 0.000

Probability for Likelihood category Negligible $=0.624$

Probability for Likelihood category Low $=0.282$

Probability for Likelihood category Medium $=0.087$

Probability for Likelihood category High $=0.008$

Probability for Likelihood category Very High $=0.000$

Probability for Consequence category Negligible $=0.700$ 
Probability for Consequence category Low $=0.300$

Probability for Consequence category Medium $=0.000$

Probability for Consequence category High $=0.000$

Probability for Consequence category Very High $=0.000$

Probability for Risk category Negligible $=0.887$

Probability for Risk category Low $=0.113$

Probability for Risk category Medium $=0.000$

Probability for Risk category High $=0.000$

Probability for Risk category Very High $=0.000$ 
Evaluation of site/facility: noinf

For scenario type: Outsider attack

Output file name: noinf4oa.ou4

Program and Version: Version 4 of OA_EVAL

Variable $\mathrm{X} 1$ is Existence of a Long Standing Conflict

$X$ 1 values are $0.500 \quad 1.000 \quad 2.000$

$\begin{array}{llll}X^{-} 1 \text { weights are } & 0.333 & 0.333 & 0.334\end{array}$

Variable $\mathrm{X} 2$ is Political/ldeological Motivations

$X \_2$ values are $0.500 \quad 1.000$

$X$ _2 weights are $0.500 \quad 0.500$

Variable X4 is Perception of Target Vulnerability

$X \quad 4$ values are $0.500 \quad 1.500$

$X$ _ 4 weights are $0.500 \quad 0.500$

Variable $X 5$ is Availability of Threat Group Resources

$X$ _5 values are $0.000 \quad 0.500 \quad 1.000 \quad 2.000$

$\begin{array}{lllll}X \_5 \text { weights are } & 0.250 & 0.250 & 0.250 & 0.250\end{array}$

Variable $X 6$ is Threat Group Training and Leadership

$X$ _6 values are $0.500 \quad 1.000 \quad 2.000$

$\begin{array}{llll}X \_6 \text { weights are } & 0.333 & 0.333 & 0.334\end{array}$

Variable $\mathrm{X} 7$ is Threat Group Knowledge and Intelligence Capability

$X$.7 values are $0.500 \quad 2.000$

$X \_7$ weights are $0.500 \quad 0.500$

Variable $X 8$ is Site/Facility Socio-Economic Conditions

$X \_8$ values are $0.750 \quad 1.000 \quad 1.500$

$\begin{array}{llll}X \_8 \text { weights are } & 0.333 & 0.333 & 0.334\end{array}$

Variable $Y 3$ is Site Protective Forces

$Y$ _3 values are $0.500 \quad 1.000 \quad 2.000$

$\begin{array}{llll}Y \_3 \text { weights are } & 0.333 & 0.333 & 0.334\end{array}$

Variable $Y 4$ is Site Intrusion Detection

$Y_{-}$4 values are $0.500 \quad 1.000 \quad 2.000$

$\begin{array}{llll}Y^{-} \text {_4 weights are } & 0.333 & 0.333 & 0.334\end{array}$

Variable $Y 5$ is Site Barriers/Delays

$Y$ Y 5 values are $0.500 \quad 1.000 \quad 2.000$

$\begin{array}{llll}Y \text { Y } 5 \text { weights are } & 0.333 & 0.333 & 0.334\end{array}$

Variable $Y 6$ is Site Access Controls

Y_6 values are $0.500 \quad 1.000 \quad 2.000$

$\begin{array}{llll}Y \text { Y 6 weights are } & 0.333 & 0.333 & 0.334\end{array}$

Variable $Y 7$ is Site Location (as it affects interdiction of diverted materials)

E.8 
$Y$ 7 values are $0.500 \quad 1.500$

$Y_{-}$7 weights are $0.500 \quad 0.500$

Variable $Y 8$ is Site Emergency Response (as it affects interdiction of diverted materials)

$Y$ Y 8 values are $0.500 \quad 1.000 \quad 2.000$

$\begin{array}{llll}Y \text { Y_8 weights are } & 0.333 & 0.333 & 0.334\end{array}$

For Threat category Negligible, the probability is 0.427

For Threat category Low, the probability is 0.268

For Threat category High, the probability is 0.184

For Threat category Very High, the probability is 0.121

For Vulnerability category Negligible, the probability is 0.232

For Vulnerability category Low, the probability is 0.232

For Vulnerability category High, the probability is 0.249

For Vulnerability category Very High, the probability is

Probability for Likelihood category Negligible $=0.367$

Probability for Likelihood category Low $=0.255$

Probability for Likelihood category Medium $=0.138$

Probability for Likelihood category High $=0.153$

Probability for Likelihood category Very High $=0.087$

Probability for Consequence category Negligible $=0.200$

Probability for Consequence category Low $=0.200$

Probability for Consequence category Medium $=0.200$

Probability for Consequence category High $=0.200$

Probability for Consequence category Very High $=0.200$

Probability for Risk Category Negligible $=0.347$

Probability for Risk Category Low $=0.251$

Probability for Risk Category Medium $=0.200$

Probability for Risk Category High $=0.154$

Probability for Risk Category Very High $=0.048$ 
Evaluation of site/facility: bigthreat_bigvuln_bigconseq_oa

For scenario type:

Output file name:

Outsider attack

Program and Version: Version 4 of OA_EVAL

Variable $\mathrm{X} 1$ is Existence of a Long Standing Conflict

$X \quad 1$ values are $0.500 \quad 1.000 \quad 2.000$

$\begin{array}{llll}X^{-} 1 \text { weights are } & 0.000 & 0.300 & 0.700\end{array}$

Variable $X 2$ is Political//deological Motivations

$X$ _2 values are $0.500 \quad 1.000$

$X \_2$ weights are $0.300 \quad 0.700$

Variable $\mathrm{X} 4$ is Perception of Target Vulnerability

$X \quad 4$ values are $0.500 \quad 1.500$

$\mathrm{X}$-4 weights are $0.300 \quad 0.700$

Variable X5 is Availability of Threat Group Resources

$X \_5$ values are $0.000 \quad 0.500 \quad 1.000 \quad 2.000$

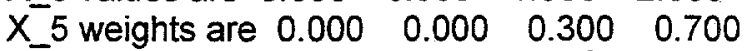

Variable $X 6$ is Threat Group Training and Leadership

$X$ 6 values are $0.500 \quad 1.000 \quad 2.000$

$\begin{array}{llll}X & -6\end{array}$ weights are $0.000 \quad 0.300 \quad 0.700$

Variable $\mathrm{X} 7$ is Threat Group Knowledge and Intelligence Capability

$X$.7 values are $0.500 \quad 2.000$

$\mathrm{X}$-7 weights are $0.300 \quad 0.700$

Variable $X 8$ is Site/Facility Socio-Economic Conditions

$X \_8$ values are $0.750 \quad 1.000 \quad 1.500$

$\mathrm{X} 8$ weights are $0.000 \quad 0.300 \quad 0.700$

Variable $Y 3$ is Site Protective Forces

$Y 3$ values are $0.500 \quad 1.000 \quad 2.000$

$\begin{array}{llll}Y \_3 & \text { weights are } 0.000 & 0.300 & 0.700\end{array}$

Variable Y4 is Site Intrusion Detection

$Y$ Y4 values are $0.500 \quad 1.000 \quad 2.000$

$\begin{array}{llll}Y_{-} \text {4 weights are } 0.000 & 0.300 & 0.700\end{array}$

Variable $Y 5$ is Site Barriers/Delays

$\begin{array}{llll}Y \text { Y } 5 \text { values are } & 0.500 & 1.000 & 2.000\end{array}$

$\begin{array}{llll}Y^{-} \text {5 weights are } & 0.000 & 0.300 & 0.700\end{array}$

Variable $Y 6$ is Site Access Controls

$Y$ Y 6 values are $0.500 \quad 1.000 \quad 2.000$

$\begin{array}{llll}Y_{-} 6 \text { weights are } & 0.000 & 0.300 & 0.700\end{array}$

Variable $Y 7$ is Site Location (as it affects interdiction of

E.10 
diverted materials)

$Y$.7 values are $0.500 \quad 1.500$

$Y_{-}^{-7}$ weights are $0.300 \quad 0.700$

Variable Y8 is Site Emergency Response (as it affects interdiction of diverted materials)

$Y$ _ 8 values are $0.500 \quad 1.000 \quad 2.000$

$\begin{array}{llll}Y & Y & 8\end{array}$

For Threat category Negligible, the probability is 0.001

For Threat category Low, the probability is 0.039

For Threat category High, the probability is 0.147

For Threat category Very High, the probability is 0.814

For Vulnerability category Negligible, the probability is 0.000

For Vulnerability category Low, the probability is 0.002

For Vulnerability category High, the probability is 0.042

For Vulnerability category Very High, the probability is 0.956

Probability for Likelihood category Negligible $=0.000$

Probability for Likelihood category Low $=0.001$

Probability for Likelihood category Medium $=0.004$

Probability for Likelihood category High $=0.077$

Probability for Likelihood category Very High $=0.918$

Probability for Consequence category Negligible $=0.000$

Probability for Consequence category Low $=0.000$

Probability for Consequence category Medium $=0.300$

Probability for Consequence category High $=0.700$

Probability for Consequence category Very High $=0.000$

Probability for Risk Category Negligible $=0.000$

Probability for Risk Category Low $=0.000$

Probability for Risk Category Medium $=0.300$

Probability for Risk Category High $=0.699$

Probability for Risk Category Very High $=0.000$ 
Evaluation of site/facility: smallthreat_smallvuln_smallconseq_oa

For scenario type:

Output file name:

Outsider attack

Program and Version: Version 4 of OA_EVAL

Variable $X 1$ is Existence of a Long Standing Conflict

$X$ _ 1 values are $0.500 \quad 1.000 \quad 2.000$

$\begin{array}{llll}X_{-} 1 \text { weights are } & 0.700 & 0.300 & 0.000\end{array}$

Variable $X 2$ is Political/ldeological Motivations

$X$ 2 values are $0.500 \quad 1.000$

$\mathrm{X} \_2$ weights are $0.700 \quad 0.300$

Variable $\mathrm{X} 4$ is Perception of Target Vulnerability

$X \quad 4$ values are $0.500 \quad 1.500$

$X \quad 4$ weights are $0.700 \quad 0.300$

Variable $X 5$ is Availability of Threat Group Resources

$\begin{array}{lllll}X & 5 \text { values are } 0.000 & 0.500 & 1.000 & 2.000\end{array}$

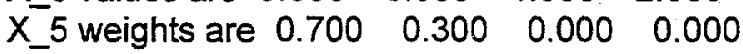

Variable $X 6$ is Threat Group Training and Leadership

$X \quad 6$ values are $0.500 \quad 1.000 \quad 2.000$

$\mathrm{X}$ 6 6 weights are $0.700 \quad 0.300 \quad 0.000$

Variable $X 7$ is Threat Group Knowledge and Intelligence Capability

$X \_7$ values are $0.500 \quad 2.000$

$X \_7$ weights are $0.700 \quad 0.300$

Variable $X 8$ is Site/Facility Socio-Economic Conditions

$X$ \& values are $0.750 \quad 1.000 \quad 1.500$

$X$ _ 8 weights are $0.700 \quad 0.300 \quad 0.000$

Variable $Y 3$ is Site Protective Forces

$Y$ Y 3 values are $0.500 \quad 1.000 \quad 2.000$

$Y_{-}$3 weights are $0.700 \quad 0.300 \quad 0.000$

Variable $Y 4$ is Site Intrusion Detection

$Y_{-} 4$ values are $0.500 \quad 1.000 \quad 2.000$

$Y_{-}^{-} 4$ weights are $0.700 \quad 0.300 \quad 0.000$

Variable $Y 5$ is Site Barriers/Delays

$Y$ Y5 values are $0.500 \quad 1.000 \quad 2.000$

$\begin{array}{llll}Y \text { _5 weights are } & 0.700 & 0.300 & 0.000\end{array}$

Variable $Y 6$ is Site Access Controls

$Y$ Y values are $0.500 \quad 1.000 \quad 2.000$

Y_6 weights are $0.700 \quad 0.300 \quad 0.000$

Variable $Y 7$ is Site Location (as it affects interdiction of

E.12 
diverted materials)

$Y$ Y 7 values are $0.500 \quad 1.500$

$\begin{array}{lll}Y_{-} & 7 \text { weights are } 0.700 \quad 0.300\end{array}$

Variable Y 8 is Site Emergency Response (as it affects interdiction of diverted materials)

$Y_{\text {_ } 8 \text { values are }} 0.500 \quad 1.000 \quad 2.000$

$\begin{array}{llll}Y_{-} \text {-8 weights are } & 0.700 & 0.300 & 0.000\end{array}$

For Threat category Negligible, the probability is 0.975

For Threat category Low, the probability is 0.024

For Threat category High, the probability is 0.001

For Threat category Very High, the probability is 0.000

For Vulnerability category Negligible, the probability is 0.977

For Vulnerability category Low, the probability is 0.023

For Vuinerability category High, the probability is 0.000

For Vuinerability category Very High, the probability is 0.000

Probability for Likelihood category Negligible $=0.999$

Probability for Likelihood category Low $=0.001$

Probability for Likelihood category Medium $=0.000$

Probability for Likelihood category High $=0.000$

Probability for Likelihood category Very High $=0.000$

Probability for Consequence category Negligible $=0.700$

Probability for Consequence category Low $=0.300$

Probability for Consequence category Medium $=0.000$

Probability for Consequence category High $=0.000$

Probability for Consequence category Very High $=0.000$

Probability for Risk Category Negligible $=1.000$

Probability for Risk Category Low $=0.000$

Probability for Risk Category Medium $=0.000$

Probability for Risk Category High $=0.000$

Probability for Risk Category Very High $=0.000$ 
Evaluation of site/facility: noinf

For scenario type: Outsider theft

Output file name: noinf4ot.ou8

Program and Version: Version 4 of OT_EVAL

Variable X4 is Perception of Target Vulnerability

$X \_4$ values are $0.500 \quad 1.500$

$X \_4$ weights are $0.500 \quad 0.500$

Variable $X 5$ is Availability of Threat Group Resources

$X$. 5 values are $0.000 \quad 0.500 \quad 1.000 \quad 2.000$

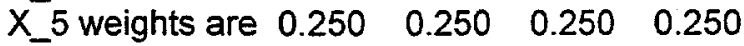

Variable $\mathrm{X} 7$ is Threat Group Knowledge and Intelligence Capability

$X$.7 values are $0.500 \quad 2.000$

$X$ _ 7 weights are $0.500 \quad 0.500$

Variable $\mathrm{X} 8$ is Site/Facility Socio-Economic Conditions

$X \quad 8$ values are $0.750 \quad 1.000 \quad 1.500$

$\begin{array}{llll}X \_8 \text { weights are } & 0.333 & 0.333 & 0.334\end{array}$

Variable $Y 2$ is Site Transfer Controls

$Y$ Y2 values are $0.500 \quad 1.000 \quad 2.000$

$\begin{array}{llll}Y \text { Y_2 weights are } & 0.333 & 0.333 & 0.334\end{array}$

Variable $Y 3$ is Site Protective Forces

$\begin{array}{llll}Y & 3 \text { values are } 0.500 & 1.000 & 2.000\end{array}$

$\begin{array}{llll}Y_{-} 3 \text { weights are } & 0.333 & 0.333 & 0.334\end{array}$

Variable $Y 4$ is Site Intrusion Detection

$Y_{\text {_ } 4 \text { values are }} 0.500 \quad 1.000 \quad 2.000$

$\begin{array}{llll}Y_{-} \text {-4 weights are } & 0.333 & 0.333 & 0.334\end{array}$

Variable $Y 5$ is Site Barriers/Delays

$\begin{array}{llll}Y \text { 5 values are } & 0.500 & 1.000 & 2.000\end{array}$

$\begin{array}{llll}Y \text { _5 weights are } & 0.333 & 0.333 & 0.334\end{array}$

Variable $Y 6$ is Site Access Controls

$Y$ Y_6 values are $0.750 \quad 1.000 \quad 2.500$

$\begin{array}{llll}Y \text { _6 weights are } & 0.333 & 0.333 & 0.334\end{array}$

Variable $Y 7$ is Site Location (as it affects interdiction of diverted materials)

$Y$ _ 7 values are $0.500 \quad 1.500$

$Y_{-}^{-7}$ weights are $0.500 \quad 0.500$

Variable $Y 8$ is Site Emergency Response (as it affects interdiction of diverted materials)

$\begin{array}{llll}Y \text { \& values are } & 0.500 & 1.000 & 2.000\end{array}$

$\begin{array}{llll}Y \text { _ } 8 \text { weights are } & 0.333 & 0.333 & 0.334\end{array}$ 
For Threat category Negligible, the probability is 0.375

For Threat category Low, the probability is 0.250

For Threat category High, the probability is 0.250

For Threat category Very High, the probability is 0.125

For Vulnerability category Negligible, the probability is 0.165

For Vulnerability category Low, the probability is 0.272

For Vulnerability category High, the probability is 0.366

For Vulnerability category Very High, the probability is 0.198

Probability for Likelihood Category Negligible $=0.342$

Probability for Likelihood Category Low $=0.204$

Probability for Likelihood Category Medium $=0.193$

Probability for Likelihood Category High $=0.187$

Probability for Likelihood Category Very High $=0.074$

Probability for Consequence category Negligible $=0.200$

Probability for Consequence category Low $=0.200$

Probability for Consequence category Medium $=0.200$

Probability for Consequence category High $=0.200$

Probability for Consequence category Very High $=0.200$

Probability for Risk category Negligible $=0.337$

Probability for Risk category Low $=0.241$

Probability for Risk category Medium $=0.200$

Probability for Risk category High $=0.170$

Probability for Risk category Very High $=0.052$ 
Evaluation of site/facility: bigthreat_bigvuln_bigconseq_ot

For scenario type:

Outsider theft

Output file name:

ot_T\&V\&C.ou8

Program and Version: Version 4 of OT_EVAL

Variable $\mathrm{X} 4$ is Perception of Target Vulnerability

$X \quad 4$ values are $0.500 \quad 1.500$

$X \_4$ weights are $0.300 \quad 0.700$

Variable X5 is Availability of Threat Group Resources

$X$ X values are $0.000 \quad 0.500 \quad 1.000 \quad 2.000$

$\begin{array}{lllll}X \text { _5 weights are } & 0.000 & 0.000 & 0.300 & 0.700\end{array}$

Variable $X 7$ is Threat Group Knowledge and Intelligence Capability

$X$ _ 7 values are $0.500 \quad 2.000$

$\mathrm{X}$ _ 7 weights are $0.300 \quad 0.700$

Variable $X 8$ is Site/Facility Socio-Economic Conditions

$X \quad 8$ values are $0.750 \quad 1.000 \quad 1.500$

$\begin{array}{llll}X \text { _8 weights are } & 0.000 & 0.300 & 0.700\end{array}$

Variable $Y 2$ is Site Transfer Controls

$Y$ _2 values are $0.500 \quad 1.000 \quad 2.000$

$\begin{array}{llll}Y \text { _. } 2 \text { weights are } 0.000 & 0.300 & 0.700\end{array}$

Variable $Y 3$ is Site Protective Forces

$Y 3$ values are $0.500 \quad 1.000 \quad 2.000$

$\begin{array}{llll}Y_{-} 3 \text { 3 weights are } & 0.000 & 0.300 & 0.700\end{array}$

Variable $Y 4$ is Site Intrusion Detection

$Y$ Y 4 values are $0.500 \quad 1.000 \quad 2.000$

$\begin{array}{llll}Y^{-} 4 \text { weights are } & 0.000 & 0.300 & 0.700\end{array}$

Variable $Y 5$ is Site Barriers/Delays

$Y$ 5 values are $0.500 \quad 1.000 \quad 2.000$

$\begin{array}{llll}Y \text { Y } 5 \text { weights are } 0.000 & 0.300 & 0.700\end{array}$

Variable $Y 6$ is Site Access Controls

$Y \quad 6$ values are $0.750 \quad 1.000 \quad 2.500$

$\begin{array}{llll}Y_{-} \text {_6 weights are } & 0.000 & 0.300 & 0.700\end{array}$

Variable $Y 7$ is Site Location (as it affects interdiction of diverted materials)

$Y$ _7 values are $0.500 \quad 1.500$

$Y^{-} 7$ weights are $0.300 \quad 0.700$

Variable Y8 is Site Emergency Response (as it affects interdiction of diverted materials)

$Y \quad 8$ values are $0.500 \quad 1.000 \quad 2.000$

$\begin{array}{llll}Y-8 \text { weights are } 0.000 & 0.300 & 0.700\end{array}$ 
For Threat category Negligible, the probability is 0.008

For Threat category Low, the probability is 0.101

For Threat category High, the probability is 0.298

For Threat category Very High, the probability is 0.593

For Vulnerability category Negligible, the probability is 0.000

For Vulnerability category Low, the probability is 0.001

For Vulnerability category High, the probability is 0.062

For Vulnerability category Very High, the probability is 0.937

Probability for Likelihood Category Negligible $=0.001$

Probability for Likelihood Category Low $=0.008$

Probability for Likelihood Category Medium $=0.007$

Probability for Likelihood Category High $=0.150$

Probability for Likelihood Category Very High $=0.835$

Probability for Consequence category Negligible $=0.000$

Probability for Consequence category Low $=0.000$

Probability for Consequence category Medium $=0.300$

Probability for Consequence category High $=0.700$

Probability for Consequence category Very High $=0.000$

Probability for Risk category Negligible $=0.000$

Probability for Risk category Low $=0.003$

Probability for Risk category Medium $=0.303$

Probability for Risk category High $=0.694$

Probability for Risk category Very High $=0.000$ 
Evaluation of site/facility: smallthreat_smallvuln_smallconseq_ot

For scenario type: Outsider theft

Output file name: ot-t-v-c.ou8

Program and Version: Version 4 of OT_EVAL

Variable $\mathrm{X} 4$ is Perception of Target Vulnerability

$X$ _4 values are $0.500 \quad 1.500$

$X$ _ 4 weights are $0.700 \quad 0.300$

Variable X5 is Availability of Threat Group Resources

$X \ldots 5$ values are $0.000 \quad 0.500 \cdot 1.000 \quad 2.000$

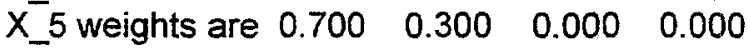

Variable $X 7$ is Threat Group Knowledge and Intelligence Capability

$X$.7 values are $0.500 \quad 2.000$

$X$ _ 7 weights are $0.700 \quad 0.300$

Variable $\mathrm{X} 8$ is Site/Facility Socio-Economic Conditions

$X \_8$ values are $0.750 \quad 1.000 \quad 1.500$

$X \_8$ weights are $0.700 \quad 0.300 \quad 0.000$

Variable $Y 2$ is Site Transfer Controls

$Y$ _2 values are $0.500 \quad 1.000 \quad 2.000$

$\begin{array}{llll}Y \text { Y_ weights are } & 0.700 & 0.300 & 0.000\end{array}$

Variable $Y 3$ is Site Protective Forces

$Y$ Y 3 values are $0.500 \quad 1.000 \quad 2.000$

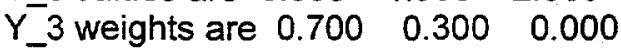

Variable Y4 is Site Intrusion Detection

$Y$ Y 4 values are $0.500 \quad 1.000 \quad 2.000$

$\begin{array}{llll}Y_{-} \text {- } 4 \text { weights are } & 0.700 & 0.300 & 0.000\end{array}$

Variable $Y 5$ is Site Barriers/Delays

$Y$ Y 5 values are $0.500 \quad 1.000 \quad 2.000$

$\begin{array}{llll}Y^{-} 5 \text { weights are } & 0.700 & 0.300 & 0.000\end{array}$

Variable $Y 6$ is Site Access Controls

Y_6 values are $0.750 \quad 1.000 \quad 2.500$

$\begin{array}{lll}Y \text { _6 weights are } 0.700 & 0.300 & 0.000\end{array}$

Variable $Y 7$ is Site Location (as it affects interdiction of diverted materials)

$Y$ Y 7 values are $0.500 \quad 1.500$

$Y_{-}$- 7 weights are $0.700 \quad 0.300$

Variable $Y 8$ is Site Emergency Response (as it affects interdiction of diverted materials)

$Y$ Y 8 values are $0.500 \quad 1.000 \quad 2.000$

$\begin{array}{lll}Y \text { _8 weights are } 0.700 & 0.300 & 0.000\end{array}$ 
For Threat category Negligible, the probability is 0.891

For Threat category Low, the probability is 0.082

For Threat category High, the probability is 0.027

For Threat category Very High, the probability is 0.000

For Vulnerability category Negligible, the probability is 0.957

For Vulnerability category Low, the probability is 0.043

For Vulnerability category High, the probability is 0.000

For Vulnerability category Very High, the probability is 0.000

Probability for Likelihood Category Negligible $=0.970$

Probability for Likelihood Category Low $=0.029$

Probability for Likelihood Category Medium $=0.001$

Probability for Likelihood Category High $=0.000$

Probability for Likelihood Category Very High $=0.000$

Probability for Consequence category Negligible $=0.700$

Probability for Consequence category Low $=0.300$

Probability for Consequence category Medium $=0.000$

Probability for Consequence category High $=0.000$

Probability for Consequence category Very High $=0.000$

Probability for Risk category Negligible $=0.991$

Probability for Risk category Low $=0.009$

Probability for Risk category Medium $=0.000$

Probability for Risk category High $=0.000$

Probability for Risk category Very High $=0.000$ 
Evaluation of site/facility: noinf

For scenario type: State transfer

Output file name: noinf4.ou6

Program and Version: Version 4 of ST_EVAL

Variable X4 is Perception of Target Vuinerability

$X \quad 4$ values are $0.500 \quad 1.500$

$X$ _4 weights are $0.500 \quad 0.500$

Variable $X 5$ is Availability of Threat Group Resources

$\begin{array}{lllll}X & 5 \text { values are } 0.000 & 0.500 & 1.000 & 2.000\end{array}$

$\begin{array}{lllll}X \_5 \text { weights are } & 0.250 & 0.250 & 0.250 & 0.250\end{array}$

Variable $X 6$ is Threat Group Training and Leadership

$X \quad 6$ values are $0.500 \quad 1.000 \quad 2.000$

$\begin{array}{llll}X \_6 \text { weights are } & 0.333 & 0.333 & 0.334\end{array}$

Variable $\mathrm{X} 7$ is Threat Group Knowledge and Intelligence Capability

$X \quad 7$ values are $0.500 \quad 1.500$

$X^{-7} 7$ weights are $0.500 \quad 0.500$

Variable $X 8$ is Site/Facility Socio-Economic Conditions

$X \_8$ values are $0.750 \quad 1.000 \quad 1.500$

$\begin{array}{llll}X \_8 \text { weights are } & 0.333 & 0.333 & 0.334\end{array}$

Variable Y9 is Overall Capability of Host Country Safeguards

Y_9 values are $0.500 \quad 1.000 \quad 2.000$

$\begin{array}{llll}Y \text { _9 weights are } & 0.333 & 0.333 & 0.334\end{array}$

Variable $Y 10$ is Host Country Socio-political Stability

$\begin{array}{llll}Y \text { 10 values are } & 0.500 & 1.000 & 2.000\end{array}$

$\begin{array}{llll}Y_{-}-10 \text { weights are } & 0.333 & 0.333 & 0.334\end{array}$

For Threat category Negligible, the probability is 0.264

For Threat category Low, the probability is 0.243

For Threat category High, the probability is 0.250

For Threat category Very High, the probability is 0.243

For Vulnerability category Negligible, the probability is 0.111

For Vulnerability category Low, the probability is 0.222

For Vulnerability category High, the probability is 0.333

For Vulnerability category Very High, the probability is 0.334

Probability for Likelihood category Negligible $=0.203$

Probability for Likelihood category Low $=0.197$

Probability for Likelihood category High $=0.190$

Probability for Likelihood category Very High $=0.246$

Probability for Likelihood category Very High $=0.165$ 
Probability for Consequence category Negligible $=0.200$

Probability for Consequence category Low $=0.200$

Probability for Consequence category Medium $=0.200$

Probability for Consequence category High $=0.200$

Probability for Consequence category Very High $=0.200$

Probability for Risk category Negligible $=0.281$

Probability for Risk category Low $=0.239$

Probability for Risk category Medium $=0.200$

Probability for Risk category High $=0.198$

Probability for Risk category Very High $=0.082$ 
Evaluation of site/facility: bigthreat_bigvuln_bigconseq_st

For scenario type: State transfer

Output file name: $\quad$ st_T\&V\&C.ou6

Program and Version: Version 4 of ST_EVAL

Variable X4 is Perception of Target Vuinerability

$X \_4$ values are $0.500 \quad 1.500$

$\mathrm{X} \_4$ weights are $0.300 \quad 0.700$

Variable $X 5$ is Availability of Threat Group Resources

$X \quad 5$ values are $0.000 \quad 0.500 \quad 1.000 \quad 2.000$

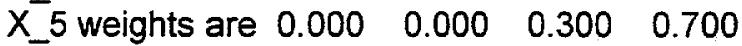

Variable $X 6$ is Threat Group Training and Leadership

$X$ _6 values are $0.500 \quad 1.000 \quad 2.000$

$\begin{array}{llll}X \text { _6 weights are } & 0.000 & 0.300 & 0.700\end{array}$

Variable $X 7$ is Threat Group Knowledge and Intelligence Capability

$X$ _ values are $0.500 \quad 1.500$

$X_{-}$- 7 weights are $0.300 \quad 0.700$

Variable $\mathrm{X} 8$ is Site/Facility Socio-Economic Conditions

$X \quad 8$ values are $0.750 \quad 1.000 \quad 1.500$

X_8 weights are $0.000 \quad 0.300 \quad 0.700$

Variable Y9 is Overall Capability of Host Country Safeguards

$Y$ Y9 values are $0.500 \quad 1.000 \quad 2.000$

$\begin{array}{llll}Y_{-} \text {-9 weights are } & 0.000 & 0.300 & 0.700\end{array}$

Variable $Y 10$ is Host Country Socio-political Stability

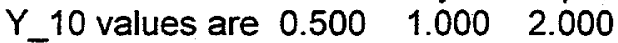

$\begin{array}{llll}Y_{-} \text {-10 weights are } & 0.000 & 0.300 & 0.700\end{array}$

For Threat category Negligible, the probability is 0.000

For Threat category Low, the probability is 0.019

For Threat category High, the probability is 0.077

For Threat category Very High, the probability is 0.903

For Vulnerability category Negligible, the probability is 0.000

For Vulnerability category Low, the probability is 0.000

For Vulnerability category High, the probability is 0.090

For Vulnerability category Very High, the probability is 0.910

Probability for Likelihood category Negligible $=0.000$

Probability for Likelihood category Low $=0.000$

Probability for Likelihood category High $=0.002$

Probability for Likelihood category Very High $=0.106$

Probability for Likelihood category Very High $=0.892$ 
Probability for Consequence category Negligible $=0.000$

Probability for Consequence category Low $=0.000$

Probability for Consequence category Medium $=0.300$

Probability for Consequence category High $=0.700$

Probability for Consequence category Very High $=0.000$

Probability for Risk category Negligible $=0.000$

Probability for Risk category Low $=0.000$

Probability for Risk category Medium $=0.300$

Probability for Risk category High $=0.700$

Probability for Risk category Very High $=0.000$ 
Evaluation of site/facility: smallthreat_smallvuin_smallconseq_st

For scenario type: State transfer

Output file name: st-t-v-c.ou6

Program and Version: Version 4 of ST_EVAL

Variable $\mathrm{X} 4$ is Perception of Target Vulnerability

$X \_4$ values are $0.500 \quad 1.500$

$X_{-} 4$ weights are $0.700 \quad 0.300$

Variable X5 is Availability of Threat Group Resources

$X$. 5 values are $0.000 \quad 0.500 \quad 1.000 \quad 2.000$

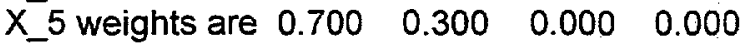

Variable $X 6$ is Threat Group Training and Leadership

$X$ _6 values are $0.500 \quad 1.000 \quad 2.000$

$\begin{array}{llll}X & 6\end{array}$ weights are $0.700 \quad 0.300 \quad 0.000$

Variable $X 7$ is Threat Group Knowledge and Intelligence Capability

$X \_7$ values are $0.500 \quad 1.500$

$X_{-7}$ weights are $0.700 \quad 0.300$

Variable $X 8$ is Site/Facility Socio-Economic Conditions

$X \quad 8$ values are $0.750 \quad 1.000 \quad 1.500$

$\begin{array}{llll}X \_ & 8 \text { weights are } 0.700 & 0.300 & 0.000\end{array}$

Variable $Y 9$ is Overall Capability of Host Country Safeguards

$Y$ _9 values are $0.500 \quad 1.000 \quad 2.000$

$\begin{array}{llll}Y \text { Y9 weights are } & 0.700 & 0.300 & 0.000\end{array}$

Variable $Y 10$ is Host Country Socio-political Stability

$Y$ _10 values are $0.500 \quad 1.000 \quad 2.000$

$\begin{array}{llll}Y_{-}-10 \text { weights are } & 0.700 & 0.300 & 0.000\end{array}$

For Threat category Negligible, the probability is 0.803

For Threat category Low, the probability is 0.183

For Threat category High, the probability is 0.014

For Threat category Very High, the probability is 0.000

For Vulnerability category Negligible, the probability is 0.490

For Vulnerability category Low, the probability is 0.420

For Vulnerability category High, the probability is 0.090

For Vulnerability category Very High, the probability is 0.000

Probability for Likelihood category Negligible $=0.893$

Probability for Likelihood category Low $=0.084$

Probability for Likelihood category High $=0.022$

Probability for Likelihood category Very High $=0.001$

Probability for Likelihood category Very High $=0.000$ 
Probability for Consequence category Negligible $=0.700$

Probability for Consequence category Low $=0.300$

Probability for Consequence category Medium $=0.000$

Probability for Consequence category High $=0.000$

Probability for Consequence category Very High $=0.000$

Probability for Risk category Negligible $=0.968$

Probability for Risk category Low $=0.032$

Probability for Risk category Medium $=0.000$

Probability for Risk category High $=0.000$

Probability for Risk category Very High $=0.000$ 
Evaluation of site/facility: noinf

For scenario type: Diversion during Transportation

Output file name: noinf $4 \times 1$.ou4

Program and version Version 4 of XP_EVAL

Variable $\mathrm{X} 1$ is Existence of a Long Standing Conflict

$\begin{array}{llll}X & 1 \text { values are } 0.500 & 1.000 & 2.000\end{array}$

$\begin{array}{llll}X & \ldots & 1\end{array}$ weights are $0.333 \quad 0.333 \quad 0.334$

Variable $X 2$ is Political/ldeological Motivations

$X \_2$ values are $0.500 \quad 1.000$

$X \_2$ weights are $0.500 \quad 0.500$

Variable $\mathrm{X} 4$ is Perception of Target Vulnerability

$X \_4$ values are $0.500 \quad 1.500$

$X 4$ weights are $0.500 \quad 0.500$

Variable $X 5$ is Availability of Threat Group Resources

$X \quad 5$ values are $0.000 \quad 0.500 \quad 1.000 \quad 2.000$

$\begin{array}{lllll}X \_5 \text { weights are } & 0.250 & 0.250 & 0.250 & 0.250\end{array}$

Variable $X 6$ is Threat Group Training and Leadership

$X \quad 6$ values are $0.500 \quad 1.000 \quad 2.000$

$\begin{array}{llll}X \text {-6 weights are } & 0.333 & 0.333 & 0.334\end{array}$

Variable $X 7$ is Threat Group Knowledge and Intelligence Capability

$X$. 7 values are $0.500 \quad 1.500$

$X \_7$ weights are $0.500 \quad 0.500$

Variable $X 8$ is Site/Facility Socio-Economic Conditions

$X \quad 8$ values are $0.750 \quad 1.000 \quad 1.500$

$\begin{array}{llll}X \_8 \text { weights are } & 0.333 & 0.333 & 0.334\end{array}$

Variable $Y_{-} 11$ measures Location Monitoring and Attack Detection

$Y$ _11 values are $0.500 \quad 1.000 \quad 2.000$

$\begin{array}{llll}Y_{-}-11 \text { weights are } & 0.333 & 0.333 & 0.334\end{array}$

Variable $Y 12$ measures Accompanying Protection Forces

$Y$ Y 12 values are $0.500 \quad 1.000 \quad 2.000$

$\begin{array}{llll}Y_{-}-12 \text { weights are } & 0.333 & 0.333 & 0.334\end{array}$

Variable $Y_{-} 13$ is Deception and Indirection

$Y$ 13 values are $0.750 \quad 1.000 \quad 1.500$

$\begin{array}{llll}Y_{-}^{-} 13 \text { weights are } 0.333 & 0.333 & 0.334\end{array}$

Variable $Y \_14$ measures Immobilization and Delay Systems

$Y$ Y 14 values are $0.500 \quad 1.000 \quad 1.250$

$\begin{array}{llll}Y & \\ Y & \end{array}$

Variable $Y_{-} 15$ measures Attack Sites and Escape Routes 
$Y$ _15 values are $0.500 \quad 1.000 \quad 2.000$

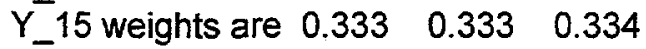

Variable $Y \_16$ measures Non-accompanying Emergency Response Forces $Y_{\text {_16 }}$ 16 values are $0.500 \quad 1.000 \quad 2.000$

$\begin{array}{llll}Y_{-}-16 \text { weights are } & 0.333 & 0.333 & 0.334\end{array}$

For Threat category Negligible, the probability is 0.386

For Threat category Low, the probability is 0.420

For Threat category High, the probability is 0.103

For Threat category Very High, the probability is 0.090

For Vulnerability category Negligible, the probability is 0.368

For Vulnerability category Low, the probability is 0.214

For Vulnerability category High, the probability is 0.216

For Vulnerability category Very High, the probability is 0.203

Probability for Likelihood category Negligible $=0.463$

Probability for Likelihood category Low $=0.239$

Probability for Likelihood category Medium $=0.132$

Probability for Likelihood category High $=0.127$

Probability for Likelihood category Very High $=0.039$

Probability for Consequence category Negligible $=0.200$

Probability for Consequence category Low $=0.200$

Probability for Consequence category Medium $=0.200$

Probability for Consequence category High $=0.200$

Probability for Consequence category Very High $=0.200$

Probability for Risk Category Negligible $=0.385$

Probability for Risk Category Low $=0.248$

Probability for Risk Category Medium $=0.200$

Probability for Risk Category High $=0.134$

Probability for Risk Category Very High $=0.033$ 
Evaluation of site/facility: bigthreat_bigvuin_bigconseq_xp

For scenario type: Diversion during Transportation

Output file name: $\quad x p \_T \& V \& C .044$

Program and version Version 4 of XP_EVAL

Variable $\mathrm{X} 1$ is Existence of a Long Standing Conflict

$X \ldots 1$ values are $0.500 \quad 1.000 \quad 2.000$

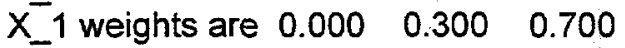

Variable $\mathrm{X} 2$ is Political//deological Motivations

$X$ 2 values are $0.500 \quad 1.000$

$\mathrm{X} \_2$ weights are $0.300 \quad 0.700$

Variable $\mathrm{X} 4$ is Perception of Target Vulnerability

$X \quad 4$ values are $0.500 \quad 1.500$

$X$ _4 weights are $0.300 \quad 0.700$

Variable X5 is Availability of Threat Group Resources

$X$.5 values are $0.000 \quad 0.500 \quad 1.000 \quad 2.000$

$\begin{array}{lllll}X \_5 \text { weights are } & 0.000 & 0.000 & 0.300 & 0.700\end{array}$

Variable $\mathrm{X} 6$ is Threat Group Training and Leadership

$X$ 6 values are $0.500 \quad 1.000 \quad 2.000$

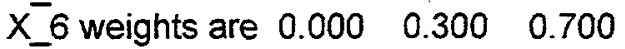

Variable $X 7$ is Threat Group Knowledge and Intelligence Capability

$X$.7 values are $0.500 \quad 1.500$

$X \_7$ weights are $0.300 \quad 0.700$

Variable $X 8$ is Site/Facility Socio-Economic Conditions

$X \_8$ values are $0.750 \quad 1.000 \quad 1.500$

$\begin{array}{llll}X & 8 \text { weights are } 0.000 & 0.300 & 0.700\end{array}$

Variable $Y_{-} 11$ measures Location Monitoring and Attack Detection

$Y$ Y_11 values are $0.500 \quad 1.000 \quad 2.000$

$\begin{array}{llll}Y^{-}-11 \text { weights are } & 0.000 & 0.300 & 0.700\end{array}$

Variable $Y_{-} 12$ measures Accompanying Protection Forces

$Y_{-}$12 values are $0.500 \quad 1.000 \quad 2.000$

$\begin{array}{llll}Y_{-}^{-} 12 \text { weights are } & 0.000 & 0.300 & 0.700\end{array}$

Variable $Y_{1} 13$ is Deception and Indirection

$Y$ 13 values are $0.750 \quad 1.000 \quad 1.500$

$\begin{array}{llll}Y_{-} \text {13 weights are } & 0.000 & 0.300 & 0.700\end{array}$

Variable Y_14 measures Immobilization and Delay Systems

$Y$ Y_14 values are $0.500 \quad 1.000 \quad 1.250$

$Y_{-}^{-} 14$ weights are $0.000 \quad 0.300 \quad 0.700$

Variable $Y_{\text {_ }} 15$ measures Attack Sites and Escape Routes

$Y$ Y_15 values are $0.500 \quad 1.000 \quad 2.000$ 
$Y_{-}$15 weights are $0.000 \quad 0.300 \quad 0.700$

Variable $Y \_16$ measures Non-accompanying Emergency Response Forces

$Y$ 16 values are $0.500 \quad 1.000 \quad 2.000$

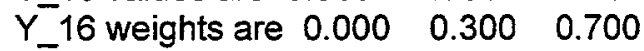

For Threat category Negligible, the probability is 0.000

For Threat category Low, the probability is 0.079

For Threat category High, the probability is 0.157

For Threat category Very High, the probability is 0.764

For Vulnerability category Negligible, the probability is 0.000

For Vulnerability category Low, the probability is 0.001

For Vulnerability category High, the probability is 0.030

For Vulnerability category Very High, the probability is 0.969

Probability for Likelihood category Negligible $=0.000$

Probability for Likelihood category Low $=0.000$

Probability for Likelihood category Medium $=0.003$

Probability for Likelihood category High $=0.104$

Probability for Likelihood category Very High $=0.893$

Probability for Consequence category Negligible $=0.000$

Probability for Consequence category Low $=0.000$

Probability for Consequence category Medium $=0.300$

Probability for Consequence category High $=0.700$

Probability for Consequence category Very High $=0.000$

Probability for Risk Category Negligible $=0.000$

Probability for Risk Category Low $=0.000$

Probability for Risk Category Medium $=0.300$

Probability for Risk Category High $=0.700$

Probability for Risk Category Very High $=0.000$ 
Evaluation of site/facility: smallthreat_smallvuln_smallconseq_xp

For scenario type:

Diversion during Transportation

Output file name:

xp-t-v-c.ou4

Program and version Version 4 of XP_EVAL

Variable $X 1$ is Existence of a Long Standing Conflict

$X$. 1 values are $0.500 \quad 1.000 \quad 2.000$

$\begin{array}{llll}X-1 \text { weights are } & 0.700 & 0.300 & 0.000\end{array}$

Variable $X 2$ is Political//deological Motivations

$X \_2$ values are $0.500 \quad 1.000$

$X \_2$ weights are $0.700 \quad 0.300$

Variable X4 is Perception of Target Vulnerability

$X$ 4 values are $0.500 \quad 1.500$

$X_{-} 4$ weights are $0.700 \quad 0.300$

Variable $X 5$ is Availability of Threat Group Resources

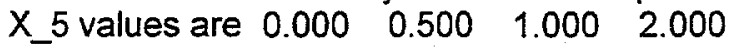

$\begin{array}{lllll}X \_5 \text { weights are } & 0.700 & 0.300 & 0.000 & 0.000\end{array}$

Variable $X 6$ is Threat Group Training and Leadership

$X$ _6 values are $0.500 \quad 1.000 \quad 2.000$

$\begin{array}{llll}X-6 \text { weights are } & 0.700 & 0.300 & 0.000\end{array}$

Variable $X 7$ is Threat Group Knowledge and Intelligence Capability

$X \quad 7$ values are $0.500 \quad 1.500$

$\mathrm{X}-7$ weights are $0.700 \quad 0.300$

Variable $X 8$ is Site/Facility Socio-Economic Conditions

$X \quad 8$ values are $0.750 \quad 1.000 \quad 1.500$

X_8 weights are $0.700 \quad 0.300 \quad 0.000$

Variable Y_11 measures Location Monitoring and Attack Detection

$Y$ _.11 values are $0.500 \quad 1.000 \quad 2.000$

$\begin{array}{llll}Y_{-}-11 \text { weights are } & 0.700 & 0.300 & 0.000\end{array}$

Variable $Y_{-} 12$ measures Accompanying Protection Forces

$Y$ _12 values are $0.500 \quad 1.000 \quad 2.000$

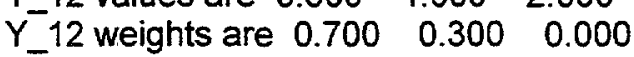

Variable $Y_{-} 13$ is Deception and Indirection

$Y$ 13 values are $0.750 \quad 1.000 \quad 1.500$

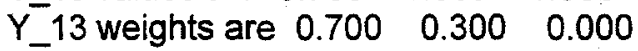

Variable $Y_{-} 14$ measures Immobilization and Delay Systems

$Y$ Y_14 values are $0.500 \quad 1.000 \quad 1.250$

$\begin{array}{llll}Y^{-} \text {-14 weights are } & 0.700 & 0.300 & 0.000\end{array}$

Variable Y_15 measures Attack Sites and Escape Routes

$Y_{\text {_ }} 15$ values are $0.500 \quad 1.000 \quad 2.000$ 
$Y_{-}$15 weights are $0.700 \quad 0.300 \quad 0.000$

Variable $Y_{-} 16$ measures Non-accompanying Emergency Response Forces

$Y$ Y_16 values are $0.500 \quad 1.000 \quad 2.000$

$\begin{array}{llll}Y_{-} \text {_16 weights are } & 0.700 & 0.300 & 0.000\end{array}$

For Threat category Negligible, the probability is 0.972

For Threat category Low, the probability is 0.028

For Threat category High, the probability is 0.000

For Threat category Very High, the probability is 0.000

For Vulnerability category Negligible, the probability is 0.998

For Vulnerability category Low, the probability is 0.002

For Vulnerability category High, the probability is 0.000

For Vulnerability category Very High, the probability is 0.000

Probability for Likelihood category Negligible $=1.000$

Probability for Likelihood category Low $=0.000$

Probability for Likelihood category Medium $=0.000$

Probability for Likelihood category High $=0.000$

Probability for Likelihood category Very High $=0.000$

Probability for Consequence category Negligible $=0.700$

Probability for Consequence category Low $=0.300$

Probability for Consequence category Medium $=0.000$

Probability for Consequence category High $=0.000$

Probability for Consequence category Very High $=0.000$

Probability for Risk Category Negligible $=1.000$

Probability for Risk Category Low $=0.000$

Probability for Risk Category Medium $=0.000$

Probability for Risk Category High $=0.000$

Probability for Risk Category Very High $=0.000$ 
Appendix F

Sample Calculation for a Simplified Model 


\title{
APPENDIX F - Sample Calculation for a Simplified Model
}

\author{
This version of the evaluation program will produce a "trace" file \\ that summarizes all of the calculations. It uses a simplified version \\ of the original ST_EVAL4 model, which has only two threat sub- \\ variables rather than the original five. It will echo the input and \\ print out statements to a "trace" file that echo each significant \\ calculational step.
}

The program has been calibrated to produce somewhat reasonable output, but no attempt has been made to have it make sense from an actual risk evaluation viewpoint. It is based on a modification of the previous State Transfer model.

The program asks the user for the name of a "trace" file. Statements summarizing and tracing the calculational steps are written out to trace file.

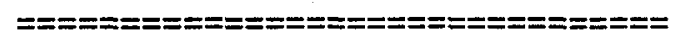

This is a prototype, with a text-based user interface. It implements the proposed threat/vulnerability measure aggregation scheme, including handling of evaluator uncertainty

The program will begin displaying the relevant threat and vulnerability sub-measures one by one and prompting the evaluator for his/her estimates of the weights (or probabilities) to be assigned to each of the possible sub-measure values. For each sub-measure, these weights should sum to 1 . The program will solicit weights for the consequence measure, which is based on the quantity and attractiveness of the nuclear materials available for diversion at the site.

When the input for all relevant sub-measures has been entered, the program will compute the aggregate values, will assign categorical values to the threat and vulnerability measures, and will compute the corresponding diversion risk measure. The evaluator-assigned uncertainties will be propagated all the way through to the diversion risk measure.

\section{DEFSNG A-H, O-Z \\ DEFINT I-N \\ OPTION BASE 1}

$\mathrm{EPS}=.001$

DIM X1(3) ' Long Standing Conflict - WEIGHTS 


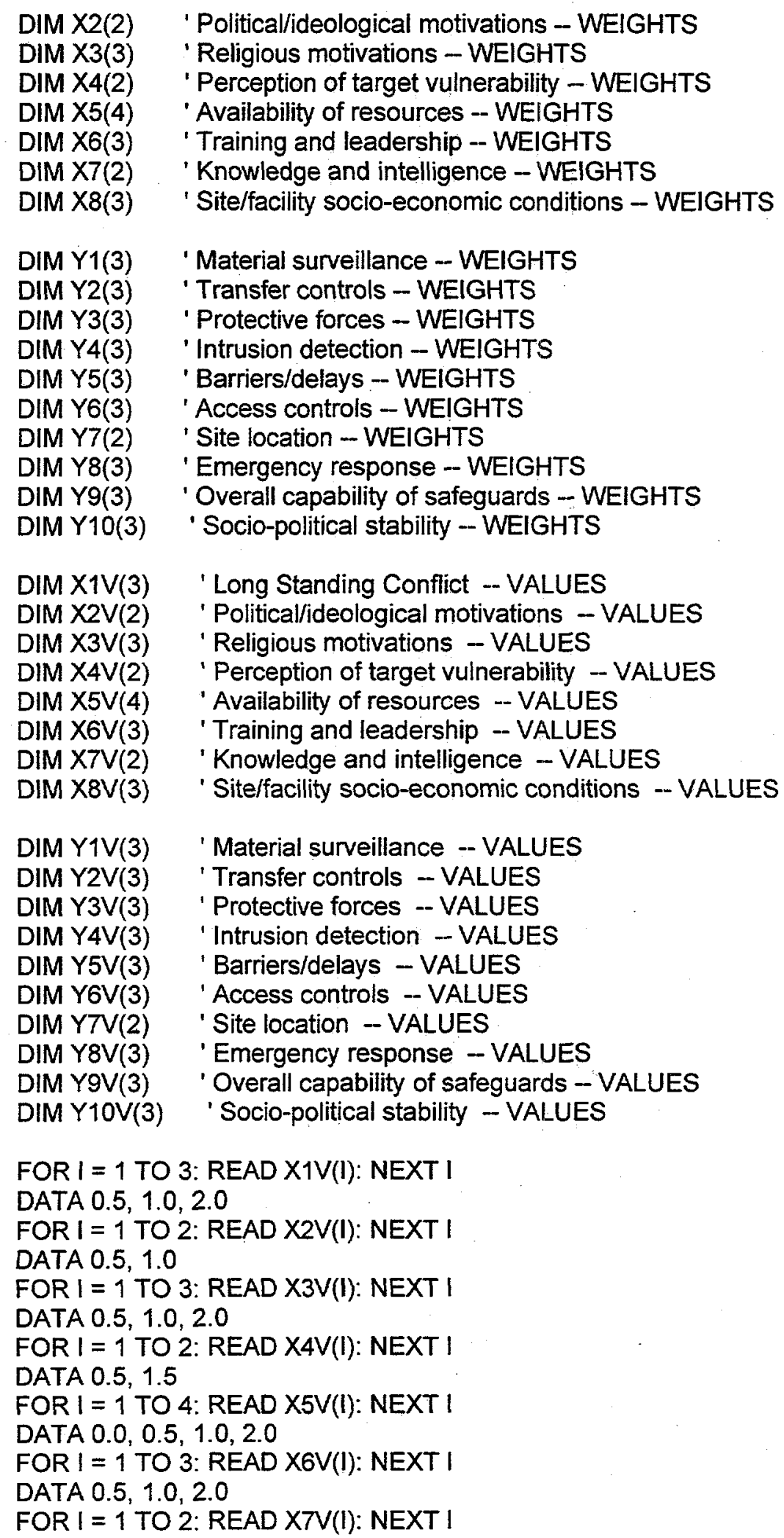


DATA $0.5,1.5$

FOR I = 1 TO 3: READ X8V(I): NEXT I

DATA $0.75,1.0,1.5$

FOR I = 1 TO 3: READ Y1V(I): NEXT I

DATA $0.5,1.0,2.0$

FOR I = 1 TO 3: READ Y2V(I): NEXT I

DATA 0.5, 1.0, 2.0

FOR I = 1 TO 3: READ Y3V(I): NEXT I

DATA 0.5, 1.0, 2.0

FOR I = 1 TO 3: READ Y4V(I): NEXT I

DATA 0.5, 1.0, 2.0

FOR I = 1 TO 3: READ Y5V(I): NEXT I

DATA 0.5, 1.0, 2.0

FOR I = 1 TO 3: READ Y6V(I): NEXT I

DATA $0.5,1.0,2.0$

FOR I = 1 TO 2: READ YTV(I): NEXT I

DATA $0.5,1.5$

FOR I = 1 TO 3: READ Y8V(I): NEXT I

DATA 0.5, 1.0, 2.0

FOR I = 1 TO 3: READ Y9V(I): NEXT I

DATA $0.5,1.0,2.0$

FOR I = 1 TO 3: READ Y $10 \mathrm{~V}(\mathrm{I}):$ NEXT I

DATA $0.5,1.0,2.0$

\section{DIM STTHREATPROB!(101)}

DIM STVULNPROB!(101)

DIM THREAT(4) 'Threat category probabilities

DIM VULN(4) 'Vulnerability category probabilities

DIM LHOOD!(5) 'Likelihood category probabilities

DIM LMAT! (4, 4) 'Likelihood matrix probabilities

DIM CONSEQ(5) 'Consequence category probabilities

DIM RISK $(5,5)$ 'Risk matrix probabilities

DIM RISKCAT(5) 'Risk category probabilities

DIM scentype\$(6) ' Scenario type names

FOR I = 1 TO 6: READ scentype\$(I): NEXT I

DATA "Insider theft", "Outsider theft", "Outsider attack", "State transfer"

DATA "Transportation", "Calculation Trace"

INPUT "Enter name of output file "; outfile\$

OPEN outfile\$ FOR OUTPUT AS \#1

INPUT "Enter site/facility name "; sitename\$

INPUT "Enter name of calculation trace file "; tracefile\$

OPEN tracefile\$ FOR OUTPUT AS \#2

- Define printout formats 
$a 2 \$=" \& \#$ \#\# \&\#.\#"

a3\$ $=$ "\&\#.\#\# \&\#.\#\# \&\#.\#"

a4\$ = "\&\#.\#\# \&\#.\#\# \&\#.\#\# \&\#.\#\#"

tv $\$=$ "\& \#\#.\#\# \&\#.\#"

C\$ = "\&\#.\#"

IC\$ = "\&\# \&\#.\#\#"

PRINT \#1,

PRINT \#1, "Evaluation of site/facility: "; sitename\$

PRINT \#1, "For scenario type: $\quad$ "; scentype\$(6)

PRINT \#1, "Output file name: "; outfile\$

PRINT \#1, "Program and Version: Version 4 of TRACE"

PRINT \#1,

PRINT \#2,

PRINT \#2, "Evaluation of site/facility: "; sitename\$

PRINT \#2, "For scenario type: $\quad$ "; scentype\$(6)

PRINT \#2, "Calculation Trace file name: "; tracefile\$

PRINT \#2, "Program and Version: Version 4 of TRACE"

PRINT \#2,

GOSUB 4000

STOP

' Subroutines 110 through 290 solicit evaluator input for Threat and

' Vulnerability sub-measures. Subroutine 100 explains that the Threat

' and Vulnerability sub-measures are all treated as random variables,

' requiring that probabilities (or weights) summing to 1 must be assigned

' to each of the numerical values taken on by that sub-measure.

100 CLS

PRINT "The Threat and Vulnerability sub-measures used in this scheme"

PRINT "for estimating the risk of diversion of nuclear materials are"

PRINT "all treated as random variables, requiring that probabilities"

PRINT "(or weights) summing to 1 must be assigned to each of the"

PRINT "numerical values taken on by that sub-measure. The input routines"

PRINT "will enforce this requirement."

PRINT '"'

PRINT "In addition, the assignment of probabilities to the sub-measure"

PRINT "can be used to represent the evaluators uncertainty about the"

PRINT "correct value of the sub-measure. For instance, in the absence of"

PRINT "any information about the true value of the sub-measure"

PRINT "(i.e., complete uncertainty), the evaluator should assign equal"

PRINT "weights to all of the sub-measure values."

PRINT '"'

PRINT "Touch any key to continue"

DO

LOOP UNTIL INKEY\$ <> "' 
PRINT \#2,

PRINT \#2, "The following statements will echo the user input"

PRINT \#2,

RETURN

110 CLS

PRINT "Existence of a Long Standing Conflict "

PRINT "'"

PRINT " X_1 $=0.5$, no underlying conflict"

PRINT " $\quad X-1=1.0$, recently developed conflict (decades-long duration)"

PRINT " $X \_1=2.0$, long standing conflict (centuries-long duration)"

PRINT "'!

PRINT "*Long Standing Conflict* is shorthand for aspects of intensity"

PRINT "and long duration in the motivation of the threat groups. It can "

PRINT "derive from extreme religious prejudice, language/cultural "

PRINT "differences, and/or from a long history of oppression, "

PRINT "suppression, or warfare between two groups of people. Examples"

PRINT "include Catholic Irish vs. Irish Protestants and the British "

PRINT "or the Basques vs. the Spanish or Islamic fundamentals vs. "

PRINT "secular Moslem governments. "

PRINT "'!

INPUT "Input three X_1 weights "; X1(1), X1(2), X1(3)

IF ABS $\left(X 1(1)+X 1(2)^{-}+X 1(3)-1 !\right)>$ EPS THEN

PRINT "Your weights for $X \_1$ should add up to 1.0."

PRINT "Please repeat the weight assignment."

PRINT "Touch any key to continue"

DO

LOOP UNTIL INKEY\$ <> "'"

GOTO 110

END IF

PRINT \#1, "Variable $X 1$ is Existence of a Long Standing Conflict"

PRINT \#1, USING a3\$; "X_1 values are "; X1V(1); " "; X1V(2); " "; X1V(3)

PRINT \#1, USING a3\$; "X_1 weights are "; X1(1); " "; X1(2); " "; X1(3)

PRINT \#1,

RETURN

$120 \mathrm{CLS}$

PRINT "Political//deological Motivations "

PRINT "'"

PRINT " X_2 $=0.5$, no political/ideological basis "

PRINT " $X \_2=1.0$, political/ideological motivations"

PRINT "'

PRINT "Examples would be support for terrorism as a component "

PRINT "of the cold war struggle between Communism and the West."

PRINT "'!

INPUT "Input two X_2 weights "; X2(1), X2(2)

IF ABS $(X 2(1)+X 2(\overline{2})-1$ !) $>$ EPS THEN

PRINT "Your weights for $X \_2$ should add up to 1.0."

PRINT "Please repeat the weight assignment."

PRINT "Touch any key to continue" 


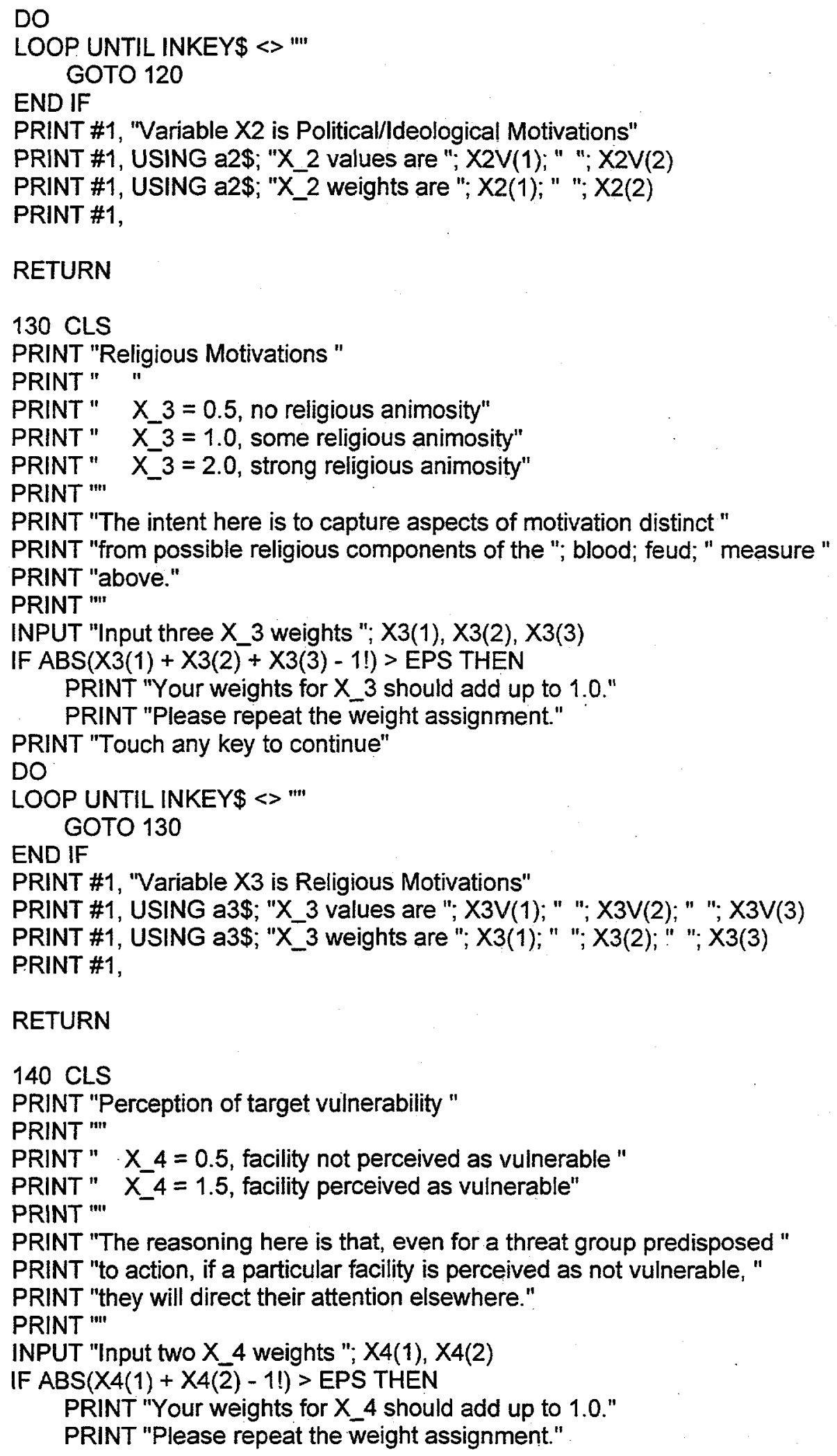




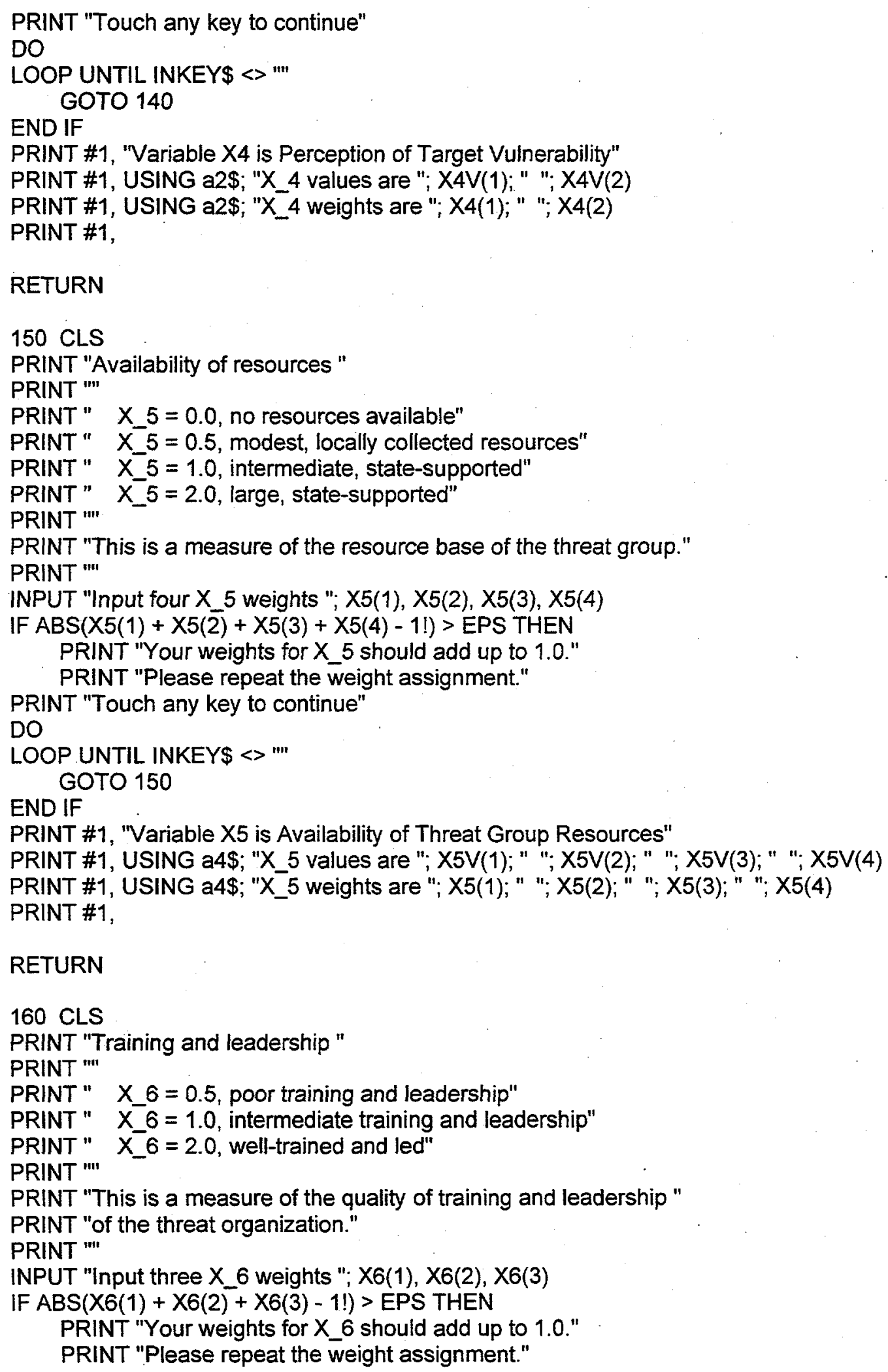


PRINT "Touch any key to continue"

DO

LOOP UNTIL INKEY\$ $<>" \cdots$ GOTO 160

END IF

PRINT \#1, "Variable X6 is Threat Group Training and Leadership"

PRINT \#1, USING a3\$; "X_6 values are "; X6V(1); " "; X6V(2); " "; X6V(3)

PRINT \#1, USING a3\$; "X_6 weights are "; X6(1); ". "; X6(2); " "; X6(3)

PRINT \#1,

\section{RETURN}

170 CLS

PRINT "Knowledge and intelligence "

PRINT "'

PRINT " $\quad X \_7=0.5$, poor intelligence capabilities"

PRINT " $X-7=1.5$, good intelligence capabilities"

PRINT "'!

PRINT "This is a measure of the access of the threat organization "

PRINT "to information about the potential target and their ability "

PRINT "to use that information."

PRINT "'!

INPUT "Input two $X \_7$ weights "; $X 7(1), X 7(2)$

IF ABS $(X 7(1)+X 7(\overline{2})-1$ !) $>$ EPS THEN

PRINT "Your weights for $X \_7$ should add up to 1.0."

PRINT "Please repeat the weight assignment."

PRINT "Touch any key to continue"

DO

LOOP UNTIL INKEY\$ $<>$ "'! GOTO 170

END IF

PRINT \#1, "Variable $X 7$ is Threat Group Knowledge and Intelligence Capability"

PRINT \#1, USING a2\$; "X_7 values are "; X7V(1); " "; X7V(2)

PRINT\#1, USING a2\$; "X_7 weights are "; $X 7(1)$; " "; $X 7(2)$

PRINT \#1,

PRINT \#2,

PRINT \#2, "Variable $X 7$ is Threat Group Knowledge and Intelligence Capability"

PRINT \#2, USING a2\$; "X_7 values are "; X7V(1); " "; X7V(2)

PRINT \#2, USING a2\$; "X_7 weights are "; $X 7(1) ;$ " "; $X 7(2)$

PRINT \#2,

RETURN

180 CLS

PRINT "Site/facility socio-economic conditions "

PRINT "'!

PRINT " X_8 $=0.75$, good conditions"

PRINT " $X \_8=1.0$, not-so-good"

PRINT " $X \_8=1.5$, extremely difficult conditions"

PRINT "'!

PRINT "This attempts to measure the likelihood that the threat "

PRINT "organization can subvert/coopt insiders to support its" 
PRINT "diversion attempt or that insiders will themselves attempt diversion." PRINT "'"

INPUT "Input three $X \_8$ weights "; $X 8(1), X 8(2), X 8(3)$

IF ABS(X8(1) + X8(2) + X8(3) - 1!) > EPS THEN

PRINT "Your weights for $X \_8$ should add up to 1.0."

PRINT "Please repeat the weight assignment."

PRINT "Touch any key to continue"

DO

LOOP UNTIL INKEY\$ <> "'"

GOTO 180

END IF

PRINT \#1, "Variable X8 is Site/Facility Socio-Economic Conditions"

PRINT \#1, USING a3\$; "X_8 values are "; X8V(1); " "; X8V(2); " "; X8V(3)

PRINT \#1, USING a3\$; "X_8 weights are "; X8(1); " "; X8(2); " "; X8(3)

PRINT \#1,

PRINT \#2,

PRINT \#2, "Variable $X 8$ is Site/Facility Socio-Economic Conditions"

PRINT \#2, USING a3S; "X_8 values are "; X8V(1); " "; X8V(2); " "; X8V(3)

PRINT \#2, USING a3\$; "X_8 weights are "; X8(1); " "; X8(2); " "; X8(3)

PRINT \#2,

\section{RETURN}

\section{CLS}

PRINT "Material Surveillance "

PRINT "'"

PRINT " $Y_{-1} 1=0.5$, excellent system of material surveillance"

PRINT " $Y^{-} 1=1.0$, good system of material surveillance"

PRINT " $\quad Y_{-}^{-} 1=2.0$, poor system of material surveillance"

PRINT "'!

PRINT "This measures the material accounting and surveillance "

PRINT "infrastructure that enables facility management to detect "

PRINT "attempted or successful diversion."

PRINT "'!

INPUT "Input three $Y$ 1 weights "; $Y 1(1), Y 1(2), Y 1(3)$

IF ABS(Y1(1) + Y1(2) + Y1(3) - 1!) > EPS THEN

PRINT "Your weights for $Y_{-} 1$ should add up to 1.0."

PRINT "Please repeat the weight assignment."

PRINT "Touch any key to continue"

DO

LOOP UNTIL INKEY\$ <> "'!

GOTO 210

END IF

PRINT \#1, "Variable $Y 1$ is Site Material Surveillance"

PRINT \#1, USING a3\$; "Y_1 values are "; Y1V(1); " "; Y1V(2); " "; Y1V(3)

PRINT \#1, USING a3\$; "Y_1 weights are "; Y1(1); " "; Y1(2); " "; Y1(3)

PRINT \#1,

\section{RETURN}

CLS 
PRINT "Transfer Controls"

PRINT "'"

PRINT " $\quad Y \_2=0.5$, excellent system of transfer control"

PRINT" Y_2 $=1.0$, good system of transfer control"

PRINT " $Y_{-} 2=2.0$, poor system of transfer control"

PRINT "'!

PRINT "This measures the security of material in transit from one "

PRINT "storage/utilization location to another."

PRINT "'"'

INPUT "Input three $Y \_2$ weights "; Y2(1), Y2(2), Y2(3)

IF ABS(Y2(1) + Y2(2) + Y2(3) - 1!) > EPS THEN

PRINT "Your weights for $Y_{-} 2$ should add up to 1.0."

PRINT "Please repeat the weight assignment."

PRINT "Touch any key to continue"

DO

LOOP UNTIL INKEY\$ $>>$ '"'

GOTO 220

END IF

PRINT \#1, "Variable Y2 is Site Transfer Controls"

PRINT \#1, USING a3\$; "Y_2 values are "; Y2V(1); ". "; Y2V(2); " "; Y2V(3)

PRINT \#1, USING a3\$; "Y_2 weights are "; Y2(1); " "; Y2(2); " "; Y2(3)

PRINT \#1,

RETURN

$230 \mathrm{CLS}$

PRINT "Protective Forces "

PRINT "'"'

PRINT " $Y_{3} 3=0.5$, excellent protective forces"

PRINT " $Y_{-} 3=1.0$, good protective forces"

PRINT " Y_3 $=2.0$, poor protective forces"

PRINT "'"

PRINT "This is a measure of the ability of the facility guard forces "

PRINT "to prevent or delay a diversion."

PRINT '"'

INPUT "Input three $Y \_3$ weights "; $Y 3(1), Y 3(2), Y 3(3)$

IF ABS $(Y 3(1)+Y 3(2)+Y 3(3)-1$ !) $>$ EPS THEN

PRINT "Your weights for $Y \_3$ should add up to 1.0."

PRINT "Please repeat the weight assignment."

PRINT "Touch any key to continue"

DO

LOOP UNTIL INKEY\$ <> "'"

GOTO 230

END IF

PRINT \#1, "Variable Y3 is Site Protective Forces"

PRINT \#1, USING a3\$; "Y_3 values are "; Y3V(1); " "; Y3V(2); " "; Y3V(3)

PRINT \#1, USING a3\$; "Y_3 weights are "; Y3(1); " "; Y3(2); " "; Y3(3)

PRINT \#1,

RETURN

240 CLS 


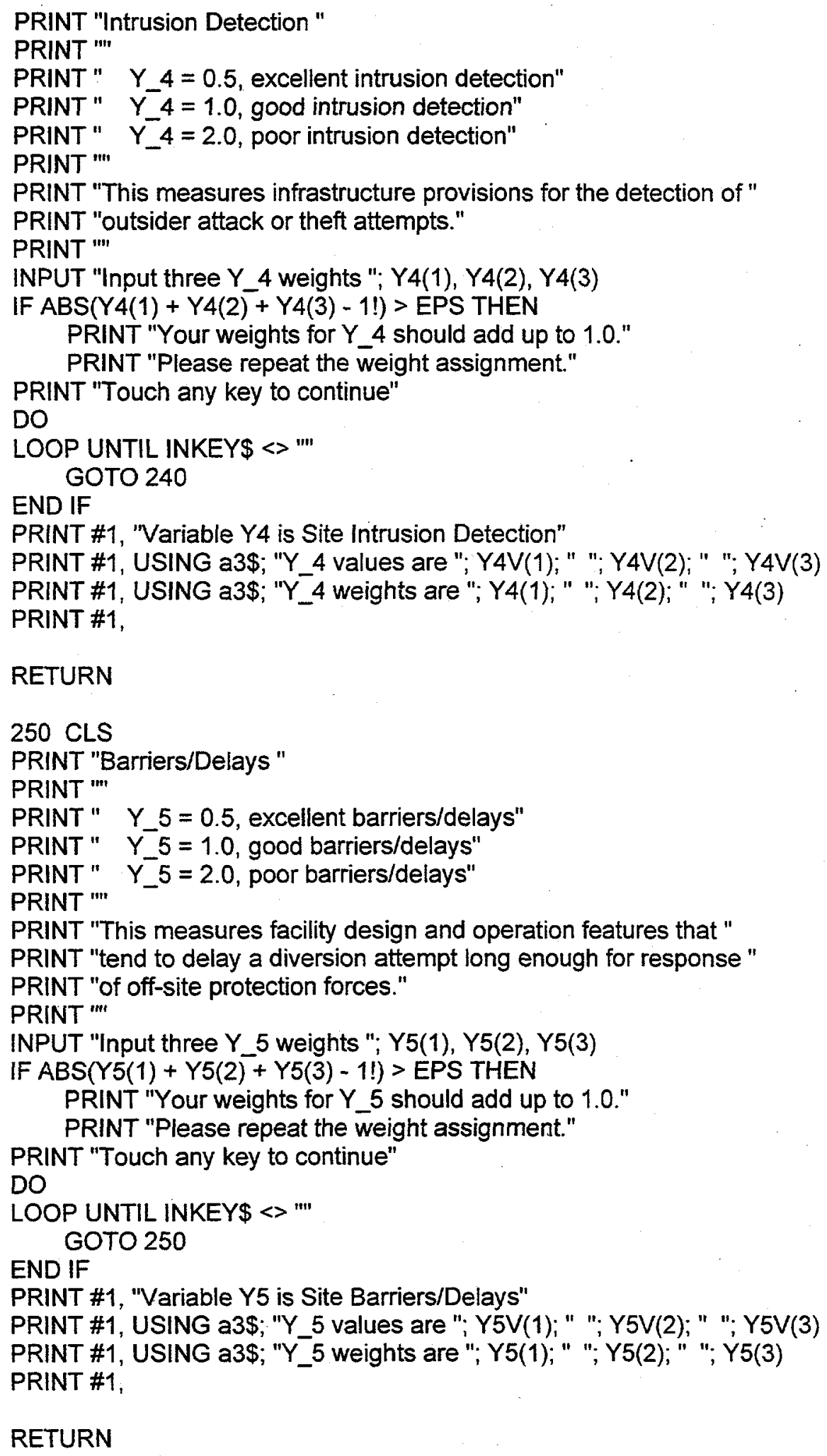


260 CLS

PRINT "Access Controls "

PRINT "'"

PRINT " Y_6 $=0.5$, excellent access controls"

PRINT " Y6 $6=1.0$, good access controls"

PRINT " Y $6=2.0$, poor access controls"

PRINT "'"

PRINT "This measures controls aimed at preventing outsider access "

PRINT "to material storage areas and to constraining the ability ".

PRINT "of insiders to gradually divert materials."

PRINT "'!

INPUT "Input three $Y$ 6 weights "; $Y 6(1), Y 6(2), Y 6(3)$

IF ABS(Y6(1) + Y6(2) + Y6(3) - 1!) > EPS THEN

PRINT "Your weights for $Y_{-} 6$ should add up to 1.0."

PRINT "Please repeat the weight assignment."

PRINT "Touch any key to continue"

DO

LOOP UNTIL INKEY\$ $<>m$

END IF

GOTO 260

PRINT \#1, "Variable Y6 is Site Access Controls"

PRINT \#1, USING a3\$; "Y_6 values are "; Y6V(1); " "; Y6V(2); " "; Y6V(3)

PRINT \#1, USING a3\$; "Y_6 weights are "; Y6(1); " "; Y6(2);" "; Y6(3)

PRINT \#1,

\section{RETURN}

\section{CLS}

PRINT "Site Location "

PRINT "'"

PRINT " $Y 7=0.5$, site with none or few good escape routes"

PRINT " $Y_{-}^{-} 7=1.5$, site with many good escape paths"

PRINT "',

PRINT "This measures the ability of group or individual that "

PRINT "has successfully diverted material to successfully remove "

PRINT "it to a secure location and/or transfer it to the end-user."

PRINT "'!

INPUT "Input two $Y 7$ weights "; $Y 7(1), Y 7(2)$

IF ABS(Y7(1) + Y7( $(2)-1$ !) $>$ EPS THEN

PRINT "Your weights for $Y_{-} 7$ should add up to 1.0."

PRINT "Please repeat the weight assignment."

PRINT "Touch any key to continue"

DO

LOOP UNTIL INKEY\$ $<>$ "'t

END IF

GOTO 270

PRINT \#1, "Variable Y7 is Site Location (as it affects interdiction of "

PRINT \#1, " diverted materials)"

PRINT \#1, USING a2\$; "Y_7 values are "; Y7V(1); " "; Y7V(2)

PRINT \#1, USING a2\$; "Y_7 weights are "; Y7(1); " "; Y7(2)

PRINT \#1, 


\section{RETURN}

\section{CLS}

PRINT "Emergency Response "

PRINT "'!

PRINT " Y $\quad 8=0.5$, site with excellent emergency response resources"

PRINT " Y_8 $=1.0$, site with some emergency response"

PRINT " Y_8 $=2.0$, site with none or poor emergency response"

PRINT "'!

PRINT "This measures the ability of off-site emergency response to "

PRINT "interdict a forcible diversion in progress, given timely "

PRINT "notification. Note that measures $Y_{-} 4, Y_{-} 5$, and $Y_{-} 7$ also"

PRINT "condition the likelihood of successful interdiction of a "

PRINT "diversion in progress."

PRINT "'

INPUT "Input three $Y$ \& 8 weights "; $Y 8(1), Y 8(2), Y 8(3)$

IF ABS(Y8(1) + Y8(2) + Y8(3) - 1!) > EPS THEN

PRINT "Your weights for $Y 8$ should add up to 1.0."

PRINT "Please repeat the weight assignment."

PRINT "Touch any key to continue"

DO

LOOP UNTIL INKEY\$ <> "'"

END IF

GOTO 280

PRINT \#1, "Variable Y8 is Site Emergency Response (as it affects" PRINT \#1," interdiction of diverted materials)"

PRINT \#1, USING a3\$; "Y_8 values are "; Y8V(1); " "; Y8V(2); " "; Y8V(3)

PRINT \#1, USING a3\$; "Y_8 weights are "; Y8(1); " "; Y8(2); " "; Y8(3)

PRINT \#1,

RETURN

285 CLS

PRINT "Overall Capability of Host Country Safeguards Measures"

PRINT "'"

PRINT " Y_9 $=0.5$, Robust safeguards measures "

PRINT " Y $9=1.0$, Safeguards with some weaknesses in design "

PRINT " $\quad$ or implementation "

PRINT " Y_9 $=2.0$, Weak or non-existent safeguards "

PRINT '"'

PRINT "This measures the overall capability of safeguards measures "

PRINT "to detect/alarm attempted state transfer of weapons material "

PRINT "to unauthorized state- or sub-state-organizations."

PRINT "m

INPUT "Input three $Y$ 9 weights "; $Y 9(1), Y 9(2), Y 9(3)$

IF ABS(Y9(1) + Y9(2) + Y9(3) - 1!) > EPS THEN

PRINT "Your weights for $Y_{-} 9$ should add up to 1.0."

PRINT "Please repeat the weight assignment."

PRINT "Touch any key to continue"

DO

LOOP UNTIL INKEY\$ <> "'"

GOTO 285 


\section{END IF}

PRINT \#1, "Variable Y9 is Overall Capability of Host Country Safeguards"

PRINT \#1, USING a3\$; "Y_9 values are "; Y9V(1); " "; Y9V(2); " "; Y9V(3)

PRINT \#1, USING a3\$; "Y_9 weights are "; Y9(1); " "; Y9(2); " "; Y9(3)

PRINT \#1,

PRINT \#2,

PRINT \#2, "Variable Y9 is Overall Capability of Host Country Safeguards"

PRINT \#2, USING a3\$; "Y_9 values are "; Y9V(1); " "; Y9V(2); " "; Y9V(3)

PRINT \#2, USING a3\$; "Y_9 weights are "; Y9(1); " "; Y9(2); " "; Y9(3)

PRINT \#2,

\section{RETURN}

290 CLS

PRINT "Socio-political stability "

PRINT "'!

PRINT " Y $10=0.5$, stable government with good foreign "

PRINT " $\quad$ relations, checks and balances"

PRINT " $\quad Y_{-} 10=1.0$, some instability, some foreign enemies, "

PRINT " - some checks and balances"

PRINT " Y $\_10=2.0$, significant instability, many factions, "

PRINT " - many enemies, no checks and balances"

PRINT "'!

PRINT "This attempts to measure the likelihood that all or part of "

PRINT "the relevant government organizations will formulate and "

PRINT "implement or will fail to impede a state transfer diversion "

PRINT "attempt. "

PRINT "'!

INPUT "Input three $Y \_10$ weights "; $Y 10(1), Y 10(2), Y 10(3)$

IF ABS $\left(Y 10(1)+Y 10 \overline{(2)}+Y_{10}(3)-1\right.$ !) > EPS THEN

PRINT "Your weights for $Y_{-} 10$ should add up to 1.0."

PRINT "Please repeat the weight assignment."

PRINT "Touch any key to continue"

DO

LOOP UNTIL INKEY\$ <> "'"

GOTO 290

\section{END IF}

PRINT \#1, "Variable Y10 is Host Country Socio-political Stability"

PRINT \#1, USING a3\$; "Y_10 values are "; Y10V(1); " "; Y10V(2); " "; Y10V(3)

PRINT\#1, USING a3\$; "Y_10 weights are "; Y10(1); " "; Y10(2); " "; Y10(3)

PRINT \#1,

PRINT \#2,

PRINT \#2, "Variable Y10 is Host Country Socio-political Stability"

PRINT \#2, USING a3\$; "Y_10 values are "; Y10V(1); " "; Y10V(2); " n; Y10V(3)

PRINT \#2, USING a3\$; "Y_10 weights are "; Y10(1); " "; Y10(2); " "; Y10(3)

PRINT \#2,

\section{RETURN}

- Subroutine 4000 calculates Threat and Vulnerability Probabilities

' for the State Transfer Theft scenario type 
4000 GOSUB 100

- GOSUB 140

- GOSUB 150

- GOSUB 160

GOSUB 170

GOSUB 180

GOSUB 285

GOSUB 290

PRINT \#2, "The following statements summarize the calculation of each of the "

PRINT \#2, "possible combinations of threat sub-variable values and the"

PRINT \#2, "probability associated with each of them.

PRINT \#2,

PRINT \#2, "In addition, the calculation of the variable STTHREATPROB!"

PRINT \#2, "accumulates the probabilities associated with particular threat"

PRINT \#2, "variable values into 100 threat variable bins, essentially"

PRINT \#2, "producing a 100 value histogram of the probabilities assigned"

PRINT \#2, "to threat variable values."

PRINT \#2,

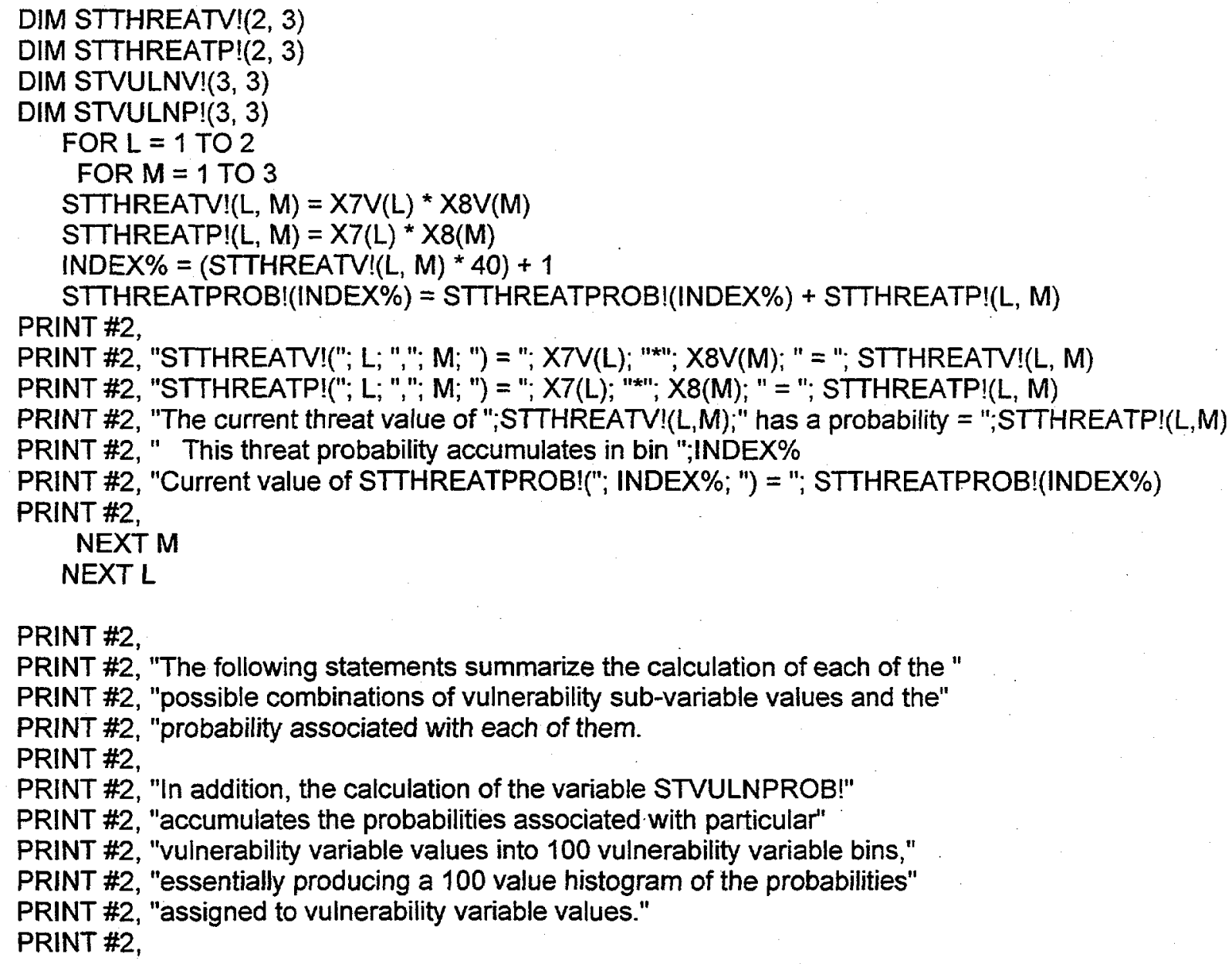

PRINT \#2,

PRINT \#2, "The following statements summarize the calculation of each of the "

PRINT \#2, "possible combinations of vulnerability sub-variable values and the"

PRINT \#2, "probability associated with each of them.

PRINT \#2,

PRINT \#2, "In addition, the calculation of the variable STVULNPROB!"

PRINT \#2, "accumulates the probabilities associated with particular"

PRINT \#2, "vulnerability variable values into 100 vulnerability variable bins,"

PRINT \#2, "essentially producing a 100 value histogram of the probabilities"

PRINT \#2, "assigned to vulnerability variable values."

PRINT \#2, 


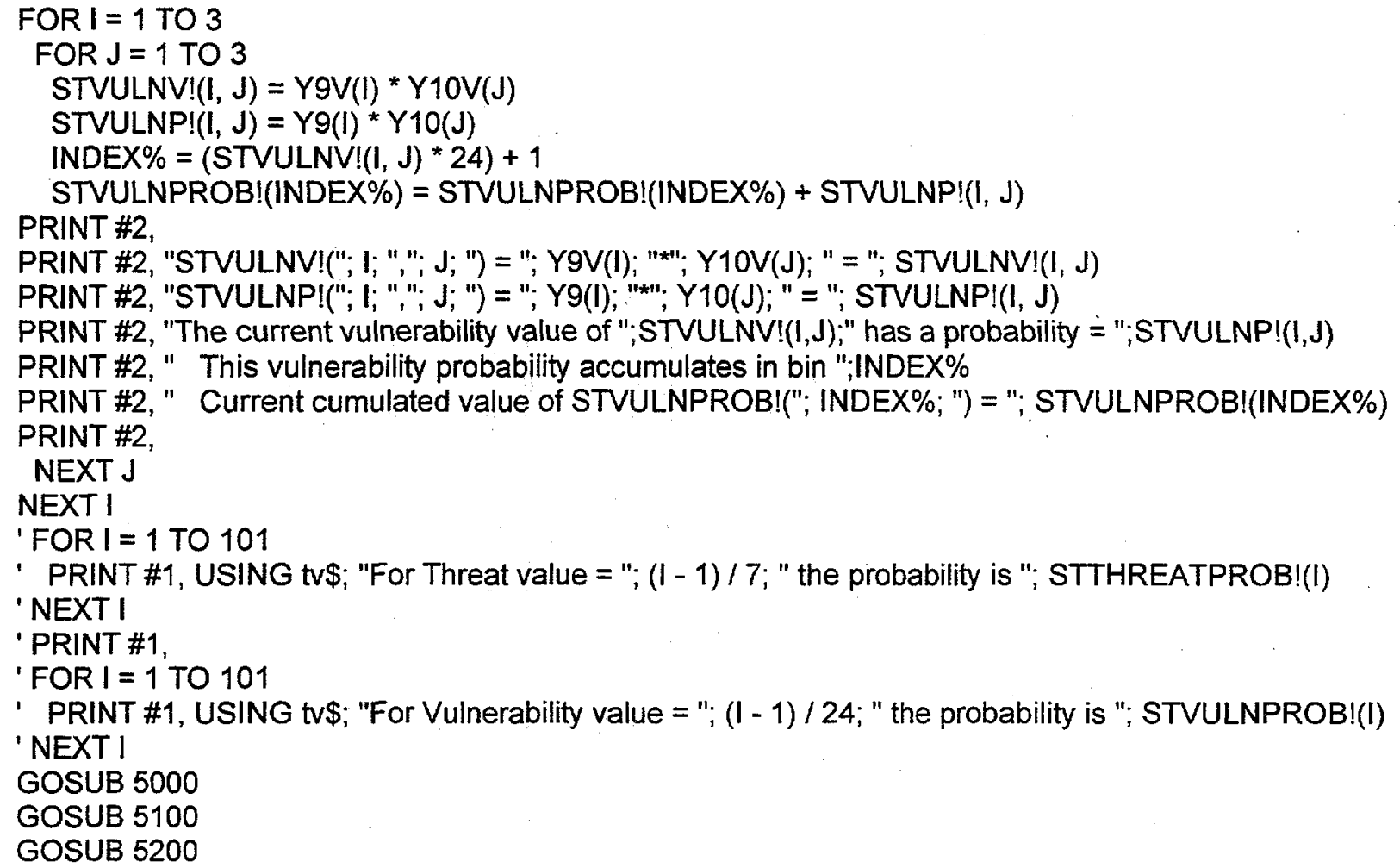

\section{RETURN}

- Subroutine 5000 accumulates the Threat probabilities values into

' four Threat category probability values for the State Transfer

' scenario type; it also accumulates Vulnerability probabilities into the

- four Vulnerability category probability values for the State Transfer

' scenario type.

5000 PRINT \#2,

PRINT \#2, "The following statements track the accumulating of the 100 threat"

PRINT \#2, "variable bins into the 4 threat variable categories."

PRINT \#2,

FOR I $=1$ TO 10

$$
\text { THREAT(1) = THREAT(1) + STTHREATPROB!(l) }
$$

PRINT \#2, "For I = ";I; "the current value of THREAT(1) = "; THREAT(1) NEXT I

PRINT \#2, FOR I $=11$ TO 20

$$
\text { THREAT(2) = THREAT(2) + STTHREATPROB!(I) }
$$

PRINT \#2, "For I = ";l; "the current value of THREAT(2) = "; THREAT(2) NEXT I

PRINT \#2,

FORI $=21$ TO 49

$$
\text { THREAT(3) = THREAT(3) + STTHREATPROB!(1) }
$$

PRINT \#2, "For 1 = ";l; "the current value of THREAT(3) = "; THREAT(3) NEXT I 


\section{PRINT \#2,}

FOR I = 50 TO 101

THREAT(4) = THREAT(4) + STTHREATPROB!(I)

PRINT \#2, "For I = ";:; "the current value of THREAT(4) = "; THREAT(4) NEXT I

PRINT \#2,

PRINT \#2, "The final values of the Threat Category probabilities are:"

PRINT \#1,

PRINT \#1, USING c\$; "For Threat category Negligible, the probability is "; THREAT(1)

PRINT \#1, USING c\$; "For Threat category Low, the probability is "; THREAT(2)

PRINT \#1, USING C\$; "For Threat category High, the probability is "; THREAT(3)

PRINT \#1, USING C\$; "For Threat category Very High, the probability is "; THREAT(4)

PRINT \#1,

PRINT \#2,

PRINT \#2, USING c\$; "For Threat category Negligible, the probability is "; THREAT(1)

PRINT \#2, USING c\$; "For Threat category Low, the probability is "; THREAT(2)

PRINT \#2, USING c\$; "For Threat category High, the probability is "; THREAT(3)

PRINT \#2, USING c\$; "For Threat category Very High, the probability is "; THREAT(4)

PRINT \#2,

PRINT \#2,

PRINT \#2, "The following statements track the accumulating of the 100 "

PRINT \#2, "vulnerability variable bins into the 4 vulnerability variable categories."

PRINT \#2,

FOR $I=1$ TO 9

VULN $(1)=$ VULN $(1)+$ STVULNPROB!(I)

PRINT \#2, "For I = "; $\mid ;$ " the current value of VULN(1) = "; VULN(1) NEXT I

PRINT \#2,

FOR $I=10$ TO 16

VULN(2) = VULN(2) + STVULNPROB!(I)

PRINT \#2, "For I = ";;; " the current value of VULN(2) = "; VULN(2) NEXT I

PRINT \#2,

FOR I $=17$ TO 30

VULN(3) $=$ VULN(3) + STVULNPROB!(I)

PRINT \#2, "For $1=$ "; 1 ; " the current value of $\operatorname{VULN}(3)=$ "; $\operatorname{VULN}(3)$ NEXT I

PRINT \#2,

FOR $\mid=31$ TO 101

$$
\text { VULN }(4)=\text { VULN }(4)+\text { STVULNPROB!(I) }
$$

PRINT \#2, "For I ="; l; " the current value of VULN(4) = "; VULN(4) NEXT I

PRINT \#2,

PRINT \#1,

PRINT \#1, USING c\$; "For Vulnerability category Negligible, the probability is "; VULN(1)

PRINT \#1, USING c\$; "For Vulnerability category Low, the probability is "; VULN(2)

PRINT \#1, USING c\$; "For Vulnerability category High, the probability is "; VULN(3)

PRINT \#1, USING C\$; "For Vulnerability category Very High, the probability is "; VULN(4)

PRINT \#1,

PRINT \#2, "The final values of the Vulnerability category probabilities are:"

PRINT \#2,

PRINT \#2, USING c\$; "For Vuinerability category Negligible, the probability is "; VULN(1) 
PRINT \#2, USING c\$; "For Vulnerability category Low, the probability is "; VULN(2) PRINT \#2, USING c\$; "For Vulnerability category High, the probability is "; VULN(3) PRINT \#2, USING c\$; "For Vulnerability category Very High, the probability is "; VULN(4) PRINT \#2, RETURN

- Subroutine 5100 computes the Likelihood matrix probability values and ' accumulates them into the five Likelihood category probabilities

PRINT \#2,

PRINT \#2, "The following statements track the calculation of the 16 probabilities"

PRINT \#2, "in the THREAT vs PROBABILITY Likelihood matrix and their accumulation" PRINT \#2, "into the five Likelihood categories."

PRINT \#2,

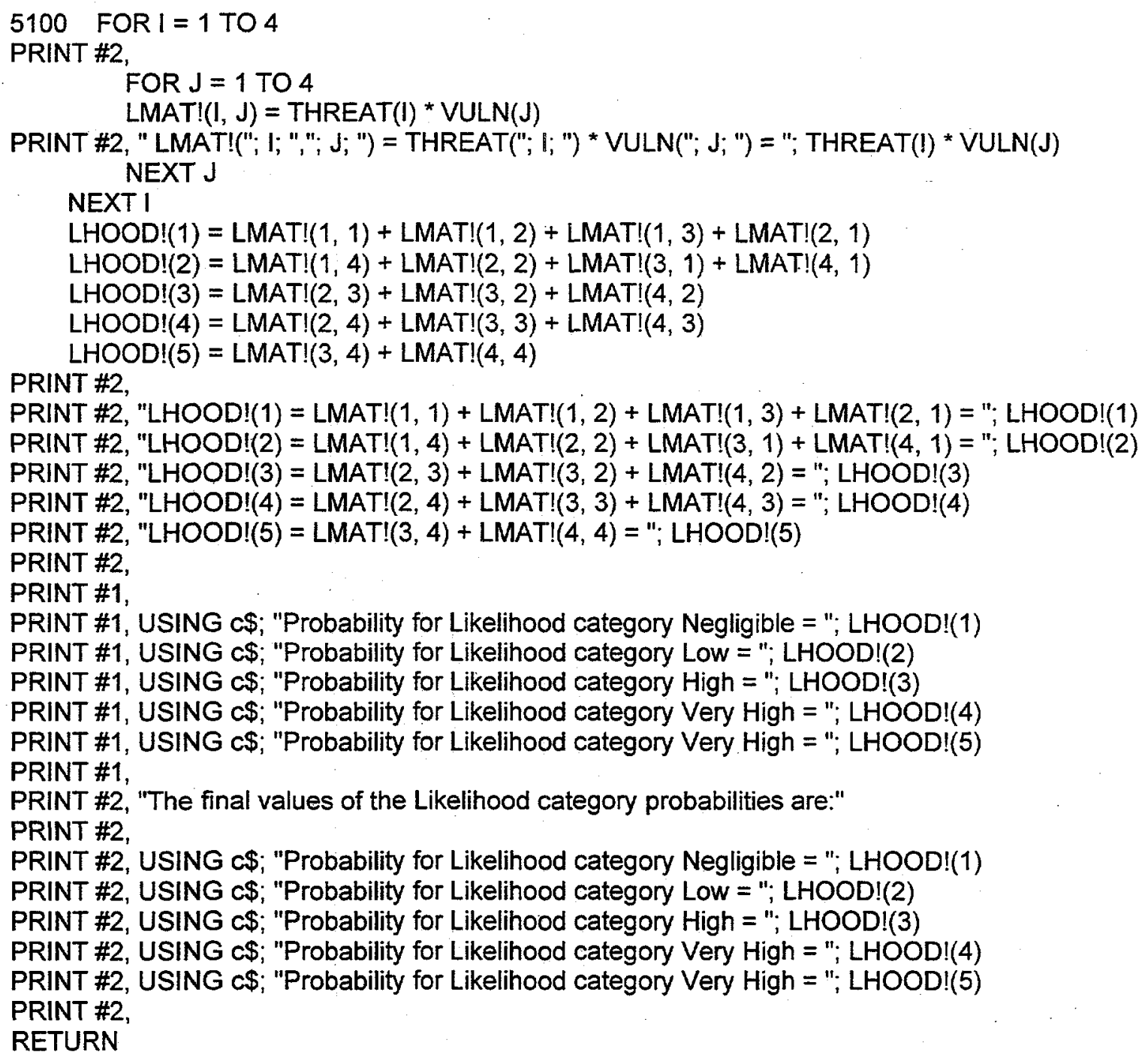


- Subroutine 5200 solicits input from the evaluator for the Consequence

- measure, given as weights/probabilities assigned to each of the five

' Consequence categories. This input is echoed to output. Finally,

' Risk Matrix probabilities are calculated and then accumulated into

' the five Risk category probabilities

5200 CLS

PRINT "Enter estimate of site/facility diversion consequences."

PRINT "Enter five weights/probabilities associated with the categories:"

PRINT "'!

PRINT " $\quad$ Negligible = Fission products or other non-weapons material"

PRINT " $\quad$ (IAEA Attractiveness Level E or less)"

PRINT "'!

PRINT "

PRINT "

PRINT "'!

PRINT "

PRINT "

PRINT "

PRINT "

PRINT "'

PRINT "

PRINT "

PRINT "

PRINT "

PRINT "'!

PRINT "

PRINT "

PRINT "'!

Low = Small amounts of difficult to convert materials"

(IAEA Attractiveness Level D, Category IV)"

Medium = Small amounts of easily convertible or large" amounts of difficult to convert materials"

(IAEA Attractiveness Level D, Categories I,II, or III"

and Attractiveness Levels B and C, Category IV)"

High = Large amounts of fissile material easily convertible" to weapons"

(IAEA Attractiveness Level B and C, "

Categories I,II, or III)"

INPUT "Input the five weights "; CONSEQ(1), CONSEQ(2), CONSEQ(3), CONSEQ(4), CONSEQ(5)

IF ABS(CONSEQ(1) + CONSEQ(2) + CONSEQ(3) + CONSEQ(4) + CONSEQ(5) - 1!) > EPS THEN

PRINT "Your weights for CONSEQ(I) should add up to 1.0."

PRINT "Please repeat the weight assignment."

PRINT "'"

PRINT "Touch any key to continue"

DO

LOOP UNTIL INKEY\$ $<>$ '"'

END IF.

GOTO 290

PRINT \#1,

PRINT \#1, USING c\$; "Probability for Consequence category Negligible = "; CONSEQ(1)

PRINT \#1, USING c\$; "Probability for Consequence category Low = "; CONSEQ(2)

PRINT \#1, USING c\$; "Probability for Consequence category Medium = "; CONSEQ(3)

PRINT \#1, USING c\$; "Probability for Consequence category High = "; CONSEQ(4)

PRINT \#1, USING c\$; "Probability for Consequence category Very High = "; CONSEQ(5)

PRINT \#1,

PRINT \#2, "The input values of the Consequence category probabilities are:"

PRINT \#2,

PRINT \#2, USING c\$; "Probability for Consequence category Negligible = "; CONSEQ(1)

PRINT \#2, USING c\$; "Probability for Consequence category Low = "; CONSEQ(2) 
PRINT \#2, USING c\$; "Probability for Consequence category Medium = "; CONSEQ(3)

PRINT \#2, USING c\$; "Probability for Consequence category High = "; CONSEQ(4)

PRINT \#2, USING c\$; "Probability for Consequence category Very High = "; CONSEQ(5)

PRINT \#2,

PRINT \#2,

PRINT \#2, "The following statements track the computation of the 25 probability"

PRINT \#2, "entries in the LIKELIHOOD vs CONSEQUENCES Risk matrix and the "

PRINT \#2, "accumulation of those probabilities into the 5 Risk categories."

PRINT \#2,

FOR I = 1 TO 5

PRINT \#2,

FOR $\mathrm{J}=1$ TO 5

RISK $(I, J)=$ LHOOD! $(I) * \operatorname{CONSEQ}(J)$

PRINT \#2, " RISK("; l; ","; J; ") = LHOOD!("; I; ") * CONSEQ("; J; ") = "; LHOOD!(I) * CONSEQ(J)

NEXT J

NEXT I

PRINT \#2,

$\operatorname{RISKCAT}(1)=\operatorname{RISK}(1,1)+\operatorname{RISK}(1,2)+\operatorname{RISK}(1,3)+\operatorname{RISK}(2,1)+\operatorname{RISK}(3,1)+\operatorname{RISK}(4,1)+\operatorname{RISK}(5,1)$

$\operatorname{RISKCAT}(2)=\operatorname{RISK}(2,3)+\operatorname{RISK}(3,2)+\operatorname{RISK}(4,2)+\operatorname{RISK}(5,2)+\operatorname{RISK}(2,2)+\operatorname{RISK}(1,4)$

$\operatorname{RISKCAT}(3)=\operatorname{RISK}(2,4)+\operatorname{RISK}(3,3)+\operatorname{RISK}(1,5)+\operatorname{RISK}(5,3)+\operatorname{RISK}(4,3)$

$\operatorname{RISKCAT}(4)=\operatorname{RISK}(2,5)+\operatorname{RISK}(3,5)+\operatorname{RISK}(3,4)+\operatorname{RISK}(4,4)+\operatorname{RISK}(5,4)$

$\operatorname{RISKCAT}(5)=\operatorname{RISK}(4,5)+\operatorname{RISK}(5,5)$

PRINT \#2, "RISKCAT(1) = RISK(1, 1) + RISK(1, 2) + RISK(1,3)+RISK(2, 1) + RISK(3, 1) + RISK(4, 1) + $\operatorname{RISK}(5,1)=$ "; RISKCAT(1)

PRINT \#2, "RISKCAT(2) $=\operatorname{RISK}(2,3)+\operatorname{RISK}(3,2)+\operatorname{RISK}(4,2)+\operatorname{RISK}(5,2)+\operatorname{RISK}(2,2)+\operatorname{RISK}(1,4)$

$=$ "; RISKCAT(2)

PRINT \#2, "RISKCAT(3) = RISK $(2,4)+\operatorname{RISK}(3,3)+\operatorname{RISK}(1,5)+\operatorname{RISK}(5,3)+\operatorname{RISK}(4,3)=$ ";

RISKCAT(3)

PRINT \#2, "RISKCAT(4) = RISK(2, 5) + RISK(3, 5) + $\operatorname{RISK}(3,4)+\operatorname{RISK}(4,4)+\operatorname{RISK}(5,4)$ = ";

RISKCAT(4)

PRINT \#2, "RISKCAT(5) = RISK $(4,5)+\operatorname{RISK}(5,5)$ = "; $\operatorname{RISKCAT}(5)$

PRINT \#2,

PRINT \#1,

PRINT \#1, USING c\$; "Probability for Risk category Negligible = "; RISKCAT(1)

PRINT \#1, USING c\$; "Probability for Risk category Low = "; RISKCAT(2)

PRINT \#1, USING c\$; "Probability for Risk category Medium = "; RISKCAT(3)

PRINT \#1, USING c\$; "Probability for Risk category High = "; RISKCAT(4)

PRINT \#1, USING c\$; "Probability for Risk category Very High = "; RISKCAT(5)

PRINT \#1,

PRINT \#2, "The final values of the Risk category probabilities are:"

PRINT \#2,

PRINT \#2, USING c\$; "Probability for Risk category Negligible = "; RISKCAT(1)

PRINT \#2, USING c\$; "Probability for Risk category Low = "; RISKCAT(2)

PRINT \#2, USING C\$; "Probability for Risk category Medium = "; RISKCAT(3)

PRINT \#2, USING c\$; "Probability for Risk category High = "; RISKCAT(4)

PRINT \#2, USING C\$; "Probability for Risk category Very High = "; RISKCAT(5)

PRINT \#2,

RETURN 


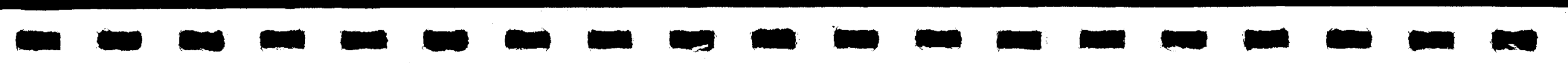


Evaluation of site/facility: TRACETST_C

For scenario type:

Output file name:

Program and Version:

Calculation Trace

TRACE4_C.OUT

Version 4 of TRACE

Variable $X 7$ is Threat Group Knowledge and Intelligence Capability

$X 7$ values are $0.500 \quad 1.500$

$X \_7$ weights are $0.300 \quad 0.700$

Variable $X 8$ is Site/Facility Socio-Economic Conditions

$X \_8$ values are $0.750 \quad 1.000 \quad 1.500$

$\begin{array}{llll}X & 8 \text { weights are } 0.200 & 0.500 & 0.300\end{array}$

Variable Y9 is Overall Capability of Host Country Safeguards

$Y 9$ values are $0.500 \quad 1.000 \quad 2.000$

$\begin{array}{llll}Y_{-} \text {99 weights are } & 0.300 & 0.600 & 0.100\end{array}$

Variable $\mathrm{Y} 10$ is Host Country Socio-political Stability

$Y \quad 10$ values are $0.500 \quad 1.000 \quad 2.000$

$\begin{array}{llll}Y_{-} \text {10 weights are } & 0.200 & 0.400 & 0.400\end{array}$

For Threat category Negligible, the probability is 0.000

For Threat category Low, the probability is 0.060

For Threat category High, the probability is 0.380

For Threat category Very High, the probability is 0.560

For Vulnerability category Negligible, the probability is 0.060

For Vulnerability category Low, the probability is 0.240

For Vulnerability category High, the probability is 0.380

For Vulnerability category Very High, the probability is 0.320

Probability for Likelihood category Negligible $=0.004$

Probability for Likelihood category Low $=0.071$

Probability for Likelihood category High $=0.248$

Probability for Likelihood category Very High $=0.376$

Probability for Likelihood category Very High $=0.301$

Probability for Consequence category Negligible $=0.000$

Probability for Consequence category Low $=0.100$

Probability for Consequence category Medium $=0.300$

Probability for Consequence category High $=0.600$

Probability for Consequence category Very High $=0.000$

Probability for Risk category Negligible $=0.001$

Probability for Risk category Low $=0.123$ 
Probability for Risk category Medium $=0.320$

Probability for Risk category High $=0.555$

Probability for Risk category Very HIgh $=0.000$ 
Evaluation of site/facility: TRACETST_C

For scenario type: Calculation Trace

Calculation Trace file name: TRACE4_C.TRC

Program and Version: Version 4 of TRACE

The following statements will echo the user input

Variable $X 7$ is Threat Group Knowledge and Intelligence Capability

$X$.7 values are $0.500 \quad 1.500$

$X \quad 7$ weights are $0.300 \quad 0.700$

Variable $X 8$ is Site/Facility Socio-Economic Conditions

$X \_8$ values are $0.750 \quad 1.000 \quad 1.500$

$\begin{array}{llll}X \_8 \text { weights are } & 0.200 & 0.500 & 0.300\end{array}$

Variable Y9 is Overall Capability of Host Country Safeguards

$Y$ Y9 values are $0.500 \quad 1.000 \quad 2.000$

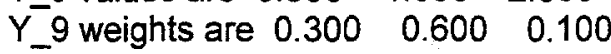

Variable $Y 10$ is Host Country Socio-political Stability

$Y$ _ 10 values are $0.500 \quad 1.000 \quad 2.000$

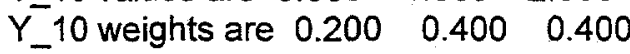

The following statements summarize the calculation of each of the possible combinations of threat sub-variable values and the probability associated with each of them.

In addition, the calculation of the variable STTHREATPROB! accumulates the probabilities associated with particular threat variable values into 100 threat variable bins, essentially producing a 100 value histogram of the probabilities assigned to threat variable values.

STTHREATV! $(1,1)=.5^{*} .75=.375$

STTHREATP! $(1,1)=.300000011920929 * .2000000029802322=6.000000238418579 \mathrm{E}-002$

The current threat value of .375 has a probability $=6.000000238418579 \mathrm{E}-002$

This threat probability accumulates in bin 16

Current value of STTHREATPROB! $(16)=6.000000238418579 \mathrm{E}-002$

STTHREATV! $(1,2)=.5 * 1=.5$

STTHREATP! $(1,2)=.300000011920929 * .5=.1500000059604645$

The current threat value of .5 has a probability $=.1500000059604645$

This threat probability accumulates in bin 21 
Current value of STTHREATPROB! $(21)=.1500000059604645$

STTHREATV! $(1,3)=.5 * 1.5=.75$

STTHREATP! $(1,3)=.300000011920929 * .300000011920929=9.000000357627869 E-002$

The current threat value of .75 has a probability $=9.000000357627869 \mathrm{E}-002$

This threat probability accumulates in bin 31

Current value of STTHREATPROB! $(31)=9.000000357627869 \mathrm{E}-002$

STTHREATV! $(2,1)=1.5 * .75=1.125$

STTHREATP! $(2,1)=.699999988079071 * .2000000029802322=.1400000005960464$

The current threat value of 1.125 has a probability $=.1400000005960464$

This threat probability accumulates in bin 46

Current value of STTHREATPROB! $(46)=.1400000005960464$

STTHREATV! $(2,2)=1.5 * 1=1.5$

STTHREATP! $(2,2)=.699999988079071 * .5=.3499999940395355$

The current threat value of 1.5 has a probability $=.3499999940395355$

This threat probability accumulates in bin 61

Current value of STTHREATPROB! $(61)=.3499999940395355$

STTHREATV! $(2,3)=1.5 * 1.5=2.25$

STTHREATP! $(2,3)=.699999988079071 * .300000011920929=.2100000083446503$

The current threat value of 2.25 has a probability $=.2100000083446503$

This threat probability accumulates in bin 91

Current value of STTHREATPROB! $(91)=.2100000083446503$

The following statements summarize the calculation of each of the possible combinations of vulnerability sub-variable values and the probability associated with each of them.

In addition, the calculation of the variable STVULNPROB! accumulates the probabilities associated with particular vulnerability variable values into 100 vulnerability variable bins, essentially producing a 100 value histogram of the probabilities assigned to vulnerability variable values.

STVULNV! $(1,1)=.5 * .5=.25$

STVULNP! $(1,1)=.300000011920929 * .2000000029802322=6.000000238418579 E-002$

The current vulnerability value of .25 has a probability $=6.000000238418579 \mathrm{E}-002$

This vulnerability probability accumulates in bin 7

Current cumulated value of STVULNPROB! 7 ) $=6.000000238418579 E-002$

STVULNV! $(1,2)=.5 * 1=.5$

STVULNP! $(1,2)=.300000011920929 * .4000000059604645=.1200000047683716$

The current vulnerability value of .5 has a probability $=.1200000047683716$ 
This vulnerability probability accumulates in bin 13

Current cumulated value of STVULNPROB! $(13)=.1200000047683716$

STVULNV! $(1,3)=.5 * 2=1$

STVULNP! $(1,3)=.300000011920929 * .4000000059604645=.1200000047683716$

The current vulnerability value of 1 has a probability $=.1200000047683716$

This vulnerability probability accumulates in bin 25

Current cumulated value of STVULNPROB! $(25)=.1200000047683716$

STVULNV! $(2,1)=1 * .5=.5$

STVULNP! $(2,1)=.6000000238418579 * .2000000029802322=.1200000047683716$

The current vulnerability value of .5 has a probability $=.1200000047683716$

This vulnerability probability accumulates in bin 13

Current cumulated value of STVULNPROB! $(13)=.2400000095367432$

STVULNV! $(2,2)=1 * 1=1$

STVULNP! $(2,2)=.6000000238418579 * .4000000059604645=.2400000095367432$

The current vulnerability value of 1 has a probability $=.2400000095367432$

This vulnerability probability accumulates in bin 25

Current cumulated value of STVULNPROB! $(25)=.3600000143051147$

STVULNV! $(2,3)=1 * 2=2$

STVULNP! $(2,3)=.6000000238418579 * .4000000059604645=.2400000095367432$

The current vulnerability value of 2 has a probability $=.2400000095367432$

This vulnerability probability accumulates in bin 49

Current cumulated value of STVULNPROB! $(49)=.2400000095367432$

STVULNV! $(3,1)=2 * .5=1$

STVULNP! $(3,1)=.1000000014901161 * .2000000029802322=2.000000141561031 \mathrm{E}-002$

The current vulnerability value of 1 has a probability $=2.000000141561031 \mathrm{E}-002$

This vulnerability probability accumulates in bin 25

Current cumulated value of STVULNPROB! $(25)=.3800000250339508$

$\operatorname{STVULNV!}(3,2)=2 * 1=2$

STVULNP! $(3,2)=.1000000014901161 * .4000000059604645=4.000000283122063 E-002$

The current vulnerability value of 2 has a probability $=4.000000283122063 \mathrm{E}-002$

This vulnerability probability accumulates in bin 49

Current cumulated value of STVULNPROB! $(49)=.2800000011920929$

STVULNV! $(3,3)=2 * 2=4$

STVULNP! $(3,3)=.1000000014901161 * .4000000059604645=4.000000283122063 E-002$

The current vuinerability value of 4 has a probability $=4.000000283122063 \mathrm{E}-002$

This vulnerability probability accumulates in bin 97

Current cumulated value of STVULNPROB! $(97)=4.000000283122063 E-002$ 
The following statements track the accumulating of the 100 threat variable bins into the 4 threat variable categories.

For $I=1$ the current value of $\operatorname{THREAT}(1)=0$

For $1=2$ the current value of THREAT(1) $=0$

For $I=3$ the current value of THREAT(1) $=0$

For $I=4$ the current value of THREAT( 1$)=0$

For $I=5$ the current value of THREAT(1) $=0$

For $I=6$ the current value of THREAT(1) $=0$

For $1=7$ the current value of THREAT $(1)=0$

For $I=8$ the current value of THREAT(1) $=0$

For $I=9$ the current value of THREAT( 1$)=0$

For $I=10$ the current value of $\operatorname{THREAT}(1)=0$

For $I=11$ the current value of $\operatorname{THREAT}(2)=0$

For $I=12$ the current value of THREAT(2) $=0$

For $I=13$ the current value of $\operatorname{THREAT}(2)=0$

For $I=14$ the current value of THREAT $(2)=0$

For $1=15$ the current value of $\operatorname{THREAT}(2)=0$

For $1=16$ the current value of THREAT( 2$)=6.000000238418579 \mathrm{E}-002$

For $I=17$ the current value of THREAT $(2)=6.000000238418579 \mathrm{E}-002$

For $l=18$ the current value of THREAT $(2)=6.000000238418579 \mathrm{E}-002$

For $1=19$ the current value of THREAT(2) $=6.000000238418579 \mathrm{E}-002$

For $1=20$ the current value of THREAT $(2)=6.000000238418579 \mathrm{E}-002$

For $\mathrm{I}=21$ the current value of THREAT(3) $=.1500000059604645$

For $I=22$ the current value of THREAT $(3)=.1500000059604645$

For $I=23$ the current value of THREAT $(3)=.1500000059604645$

For $1=24$ the current value of THREAT $(3)=.1500000059604645$

For $1=25$ the current value of THREAT $(3)=.1500000059604645$

For $I=26$ the current value of THREAT $(3)=.1500000059604645$

For $l=27$ the current value of THREAT $(3)=.1500000059604645$

For $\mathrm{I}=28$ the current value of THREAT $(3)=.1500000059604645$

For $I=29$ the current value of THREAT(3) $=.1500000059604645$

For I $=30$ the current value of THREAT $(3)=.1500000059604645$

For I $=31$ the current value of THREAT $(3)=.2400000095367432$

For $I=32$ the current value of THREAT $(3)=.2400000095367432$

For I $=33$ the current value of THREAT(3) $=.2400000095367432$

For $I=34$ the current value of $\operatorname{THREAT}(3)=.2400000095367432$

For I $=35$ the current value of THREAT $(3)=.2400000095367432$

For $\mathrm{l}=36$ the current value of THREAT( 3$)=.2400000095367432$

For $\mathrm{I}=37$ the current value of THREAT(3) $=.2400000095367432$

For $\mathrm{I}=38$ the current value of THREAT(3) $=.2400000095367432$

For I $=39$ the current value of THREAT(3) $=.2400000095367432$

For I $=40$ the current value of THREAT $(3)=.2400000095367432$

For I $=41$ the current value of THREAT $(3)=.2400000095367432$

For $\mathrm{I}=42$ the current value of THREAT( 3$)=.2400000095367432$

For $\mathrm{I}=43$ the current value of THREAT(3) $=.2400000095367432$

For $\mathrm{I}=44$ the current value of THREAT $(3)=.2400000095367432$

For $I=45$ the current value of THREAT(3) $=.2400000095367432$

For I $=46$ the current value of THREAT(3) $=.3799999952316284$ 
For I $=47$ the current value of THREAT(3) $=.3799999952316284$

For I $=48$ the current value of THREAT(3) $=.3799999952316284$

For $\mathrm{I}=49$ the current value of THREAT $(3)=.3799999952316284$

For $I=50$ the current value of $\operatorname{THREAT}(4)=0$

For $1=51$ the current value of $\operatorname{THREAT}(4)=0$

For $l=52$ the current value of THREAT(4) $=0$

For $I=53$ the current value of $\operatorname{THREAT}(4)=0$

For $1=54$ the current value of THREAT(4) $=0$

For $I=55$ the current value of THREAT(4) $=0$

For $1=56$ the current value of THREAT $(4)=0$

For $I=57$ the current value of THREAT(4) $=0$

For $1=58$ the current value of THREAT(4) $=0$

For $1=59$ the current value of THREAT(4) $=0$

For $I=60$ the current value of THREAT(4) $=0$

For $I=61$ the current value of THREAT(4) $=.3499999940395355$

For $\mathrm{I}=62$ the current value of THREAT(4) $=.3499999940395355$

For $1=63$ the current value of THREAT(4) $=.3499999940395355$

For $1=64$ the current value of THREAT(4) $=.3499999940395355$

For $I=65$ the current value of THREAT(4) $=.3499999940395355$

For $I=66$ the current value of THREAT(4) $=.3499999940395355$

For $1=67$ the current value of THREAT(4) $=.3499999940395355$

For $I=68$ the current value of THREAT(4) $=.3499999940395355$

For $I=69$ the current value of THREAT(4) $=.3499999940395355$

For $1=70$ the current value of THREAT(4) $=.3499999940395355$

For $I=71$ the current value of THREAT(4) $=.3499999940395355$

For I $=72$ the current value of THREAT(4) $=.3499999940395355$

For I $=73$ the current value of THREAT(4) $=.3499999940395355$

For I $=74$ the current value of THREAT(4) $=.3499999940395355$

For $\mathrm{I}=75$ the current value of THREAT(4) $=.3499999940395355$

For I $=76$ the current value of THREAT(4) $=.3499999940395355$

For $I=77$ the current value of THREAT(4) $=.3499999940395355$

For I $=78$ the current value of THREAT(4) $=.3499999940395355$

For I $=79$ the current value of THREAT(4) $=.3499999940395355$

For $I=80$ the current value of THREAT(4) $=.3499999940395355$

For $I=81$ the current value of THREAT(4) $=.3499999940395355$

For $\mathrm{I}=82$ the current value of THREAT $(4)=.3499999940395355$

For $I=83$ the current value of THREAT(4) $=.3499999940395355$

For $I=84$ the current value of THREAT(4) $=.3499999940395355$

For $\mathrm{I}=85$ the current value of THREAT(4) $=.3499999940395355$

For $I=86$ the current value of THREAT(4) $=.3499999940395355$

For $I=87$ the current value of THREAT(4) $=.3499999940395355$

For $I=88$ the current value of THREAT(4) $=.3499999940395355$

For $\mathrm{I}=89$ the current value of THREAT(4) $=.3499999940395355$

For $I=90$ the current value of THREAT(4) $=.3499999940395355$

For $I=91$ the current value of THREAT(4) $=.5600000023841858$

For $I=92$ the current value of THREAT(4) $=.5600000023841858$

For $\mathrm{I}=93$ the current value of THREAT(4) $=.5600000023841858$

For $\mathrm{I}=94$ the current value of THREAT(4) $=.5600000023841858$

For $\mathrm{I}=95$ the current value of THREAT(4) $=.5600000023841858$

For $I=96$ the current value of THREAT(4) $=.5600000023841858$

For $I=97$ the current value of THREAT(4) $=.5600000023841858$ 
For $1=98$ the current value of THREAT(4) $=.5600000023841858$

For $l=99$ the current value of THREAT $(4)=.5600000023841858$

For $I=100$ the current value of THREAT $(4)=.5600000023841858$

For $I=101$ the current value of THREAT $(4)=.5600000023841858$

The final values of the Threat Category probabilities are:

For Threat category Negligible, the probability is 0.000

For Threat category Low, the probability is 0.060

For Threat category High, the probability is 0.380

For Threat category Very High, the probability is 0.560

The following statements track the accumulating of the 100

vulnerability variable bins into the 4 vulnerability variable categories.

For I $=1$ the current value of $\operatorname{VULN}(1)=0$

For I $=2$ the current value of VULN $(1)=0$

For I $=3$ the current value of VULN(1) $=0$

For $I=4$ the current value of $\operatorname{VULN}(1)=0$

For $I=5$ the current value of VULN $(1)=0$

For $1=6$ the current value of $\operatorname{VULN}(1)=0$

For $I=7$ the current value of VULN $(1)=6.000000238418579 E-002$

For $I=8$ the current value of VULN $(1)=6.000000238418579 \mathrm{E}-002$

For I $=9$ the current value of VULN(1) $=6.000000238418579 \mathrm{E}-002$

For $I=10$ the current value of $\operatorname{VULN}(2)=0$

For I $=11$ the current value of $\operatorname{VULN}(2)=0$

For $I=12$ the current value of VULN(2) $=0$

For $I=13$ the current value of VULN $(2)=.2400000095367432$

For I $=14$ the current value of VULN(2) $=.2400000095367432$

For I $=15$ the current value of VULN $(2)=.2400000095367432$

For I $=16$ the current value of VULN $(2)=.2400000095367432$

For $i=17$ the current value of $\operatorname{VULN}(3)=0$

For $I=18$ the current value of VULN $(3)=0$

For $I=19$ the current value of $\operatorname{VULN}(3)=0$

For $I=20$ the current value of $\operatorname{VULN}(3)=0$

For I $=21$ the current value of VULN $(3)=0$

For $1=22$ the current value of VULN $(3)=0$

For $1=23$ the current value of VULN $(3)=0$

For $1=24$ the current value of $\operatorname{VULN}(3)=0$

For $1=25$ the current value of VULN $(3)=.3800000250339508$

For $I=26$ the current value of VULN(3) $=.3800000250339508$

For $1=27$ the current value of VULN(3) $=.3800000250339508$

For $1=28$ the current value of VULN $(3)=.3800000250339508$

For $\mathrm{I}=29$ the current value of VULN(3) $=.3800000250339508$

For $I=30$ the current value of VULN $(3)=.3800000250339508$

For I $=31$ the current value of VULN $(4)=0$

For $1=32$ the current value of $\operatorname{VULN}(4)=0$

For I $=33$ the current value of VULN $(4)=0$ 
For $1=34$ the current value of $\operatorname{VULN}(4)=0$

For $1=35$ the current value of VULN(4) $=0$

For $1=36$ the current value of $\operatorname{VULN}(4)=0$

For $1=37$ the current value of VULN $(4)=0$

For $I=38$ the current value of $\operatorname{VULN}(4)=0$

For $1=39$ the current value of $\operatorname{VULN}(4)=0$

For $I=40$ the current value of $\operatorname{VULN}(4)=0$

For $1=41$ the current value of VULN(4) $=0$

For $1=42$ the current value of VULN $(4)=0$

For $I=43$ the current value of VULN $(4)=0$

For I $=44$ the current value of VULN(4) $=0$

For I $=45$ the current value of VULN $(4)=0$

For I $=46$ the current value of VULN $(4)=0$

For $I=47$ the current value of VULN(4) $=0$

For $I=48$ the current value of VULN $(4)=0$

For $I=49$ the current value of VULN $(4)=.2800000011920929$

For $\mathrm{I}=50$ the current value of VULN(4) $=.2800000011920929$

For I $=51$ the current value of VULN(4) $=.2800000011920929$

For $\mathrm{I}=52$ the current value of VULN(4) $=.2800000011920929$

For $I=53$ the current value of VULN(4) $=.2800000011920929$

For $I=54$ the current value of VULN(4) $=.2800000011920929$

For $\mathrm{I}=55$ the current value of VULN(4) $=.2800000011920929$

For $\mathrm{I}=56$ the current value of VULN(4) $=.2800000011920929$

For $I=57$ the current value of VULN(4) $=.2800000011920929$

For $I=58$ the current value of VULN $(4)=.2800000011920929$

For $1=59$ the current value of VULN $(4)=.2800000011920929$

For $I=60$ the current value of VULN(4) $=.2800000011920929$

For $\mathrm{I}=61$ the current value of VULN $(4)=.2800000011920929$

For $\mathrm{I}=62$ the current value of VULN $(4)=.2800000011920929$

For $I=63$ the current value of VULN $(4)=.2800000011920929$

For $\mathrm{I}=64$ the current value of VULN $(4)=.2800000011920929$

For $I=65$ the current value of VULN $(4)=.2800000011920929$

For $\mathrm{I}=66$ the current value of VULN(4) $=.2800000011920929$

For $\mathrm{I}=67$ the current value of VULN(4) $=.2800000011920929$

For $I=68$ the current value of VULN $(4)=.2800000011920929$

For $1=69$ the current value of VULN(4) $=.2800000011920929$

For $1=70$ the current value of VULN(4) $=.2800000011920929$

For $I=71$ the current value of VULN(4) $=.2800000011920929$

For $1=72$ the current value of VULN(4) $=.2800000011920929$

For $1=73$ the current value of VULN(4) $=.2800000011920929$

For $I=74$ the current value of VULN(4) $=.2800000011920929$

For $I=75$ the current value of VULN $(4)=.2800000011920929$

For $\mathrm{I}=76$ the current value of VULN $(4)=.2800000011920929$

For $1=77$ the current value of VULN $(4)=.2800000011920929$

For $\mathrm{I}=78$ the current value of VULN(4) $=.2800000011920929$

For $\mathrm{I}=79$ the current value of VULN(4) $=.2800000011920929$

For $\mathrm{I}=80$ the current value of VULN $(4)=.2800000011920929$

For $I=81$ the current value of VULN (4) $=.2800000011920929$

For $I=82$ the current value of VULN(4) $=.2800000011920929$

For $I=83$ the current value of VULN(4) $=.2800000011920929$

For $1=84$ the current value of VULN $(4)=.2800000011920929$

For $\mathrm{l}=85$ the current value of VULN(4) $=.2800000011920929$ 
For $I=86$ the current value of VULN(4) $=.2800000011920929$

For $I=87$ the current value of VULN(4) $=.2800000011920929$

For I $=88$ the current value of VULN(4) $=.2800000011920929$

For I $=89$ the current value of VULN(4) $=.2800000011920929$

For I $=90$ the current value of VULN(4) $=.2800000011920929$

For I $=91$ the current value of VULN(4) $=.2800000011920929$

For $\mathrm{I}=92$ the current value of VULN(4) $=.2800000011920929$

For I $=93$ the current value of VULN(4) $=.2800000011920929$

For I $=94$ the current value of VULN(4) $=.2800000011920929$

For $I=95$ the current value of VULN(4) $=.2800000011920929$

For $\mathrm{I}=96$ the current value of VULN(4) $=.2800000011920929$

For I $=97$ the current value of VULN(4) $=.3199999928474426$

For I $=98$ the current value of VULN(4) $=.3199999928474426$

For $\mathrm{l}=99$ the current value of VULN(4) $=.3199999928474426$

For $I=100$ the current value of VULN $(4)=.3199999928474426$

For I $=101$ the current value of VULN(4) $=.3199999928474426$

The final values of the Vulnerability category probabilities are:

For Vulnerability category Negligible, the probability is 0.060

For Vulnerability category Low, the probability is 0.240

For Vulnerability category High, the probability is 0.380

For Vulnerability category Very High, the probability is 0.320

$\operatorname{LMAT}(1,1)=\operatorname{THREAT}(1) * \operatorname{VULN}(1)=0$
$\operatorname{LMAT!}(1,2)=\operatorname{THREAT}(1) * \operatorname{VULN}(2)=0$
$\operatorname{LMAT}(1,3)=\operatorname{THREAT}(1) * \operatorname{VULN}(3)=0$
$\operatorname{LMAT}(1,4)=\operatorname{THREAT}(1) * \operatorname{VULN}(4)=0$

LMAT! $(2,1)=$ THREAT( 2$) * \operatorname{VULN}(1)=3.600000286102301 E-003$

LMAT! $(2,2)=$ THREAT $(2) * \operatorname{VULN}(2)=1.44000011444092 \mathrm{E}-002$

LMAT! $(2,3)=$ THREAT( 2$) * \operatorname{VULN}(3)=2.280000240802771 \mathrm{E}-002$

LMAT! $(2,4)=$ THREAT $(2) * \operatorname{VULN}(4)=1.920000033378599 \mathrm{E}-002$

LMAT! $(3,1)=$ THREAT $(3) * \operatorname{VULN}(1)=2.280000061988829 \mathrm{E}-002$

LMAT! $(3,2)=$ THREAT ( 3$) * \operatorname{VULN}(2)=9.120000247955318 \mathrm{E}-002$

LMAT! $(3,3)=$ THREAT $(3) *$ VULN $(3)=.14440000770092$

LMAT! $(3,4)=$ THREAT $(3) * \operatorname{VULN}(4)=.1215999957561493$

LMAT! $(4,1)=$ THREAT $(4) * \operatorname{VULN}(1)=3.36000014781952 \mathrm{E}-002$

LMAT! $(4,2)=$ THREAT $(4) * \operatorname{VULN}(2)=.1344000059127808$

LMAT! $(4,3)=$ THREAT $(4) * \operatorname{VULN}(3)=.2128000149250031$

LMAT! $(4,4)=$ THREAT $(4) * \operatorname{VULN}(4)=.1791999967575073$

LHOOD!(1) = LMAT! $(1,1)+\operatorname{LMAT!}(1,2)+\operatorname{LMAT} !(1,3)+\operatorname{LMAT} !(2,1)=3.600000170990825 E-003$

LHOOD! $(2)=\operatorname{LMAT}(1,4)+\operatorname{LMAT}(2,2)+\operatorname{LMAT} !(3,1)+\operatorname{LMAT}(4,1)=7.080000638961792 \mathrm{E}-002$

LHOOD! $(3)=$ LMAT! $(2,3)+\operatorname{LMAT}(3,2)+\operatorname{LMAT} !(4,2)=.248400017619133$

LHOOD! $(4)=$ LMAT! $(2,4)+$ LMAT $(3,3)+$ LMAT! $(4,3)=.3764000236988068$

LHOOD! $(5)=\operatorname{LMAT} !(3,4)+\operatorname{LMAT} !(4,4)=.3007999956607819$

The final values of the Likelihood category probabilities are: 
Probability for Likelihood category Negligible $=0.004$

Probability for Likelihood category Low $=0.071$

Probability for Likelihood category High $=0.248$

Probability for Likelihood category Very High $=0.376$

Probability for Likelihood category Very High $=0.301$

The input values of the Consequence category probabilities are:

Probability for Consequence category Negligible $=0.000$

Probability for Consequence category Low $=0.100$

Probability for Consequence category Medium $=0.300$

Probability for Consequence category High $=0.600$

Probability for Consequence category Very High $=0.000$

The following statements track the computation of the 25 probability entries in the LIKELIHOOD vs CONSEQUENCES Risk matrix and the accumulation of those probabilities into the 5 Risk categories.

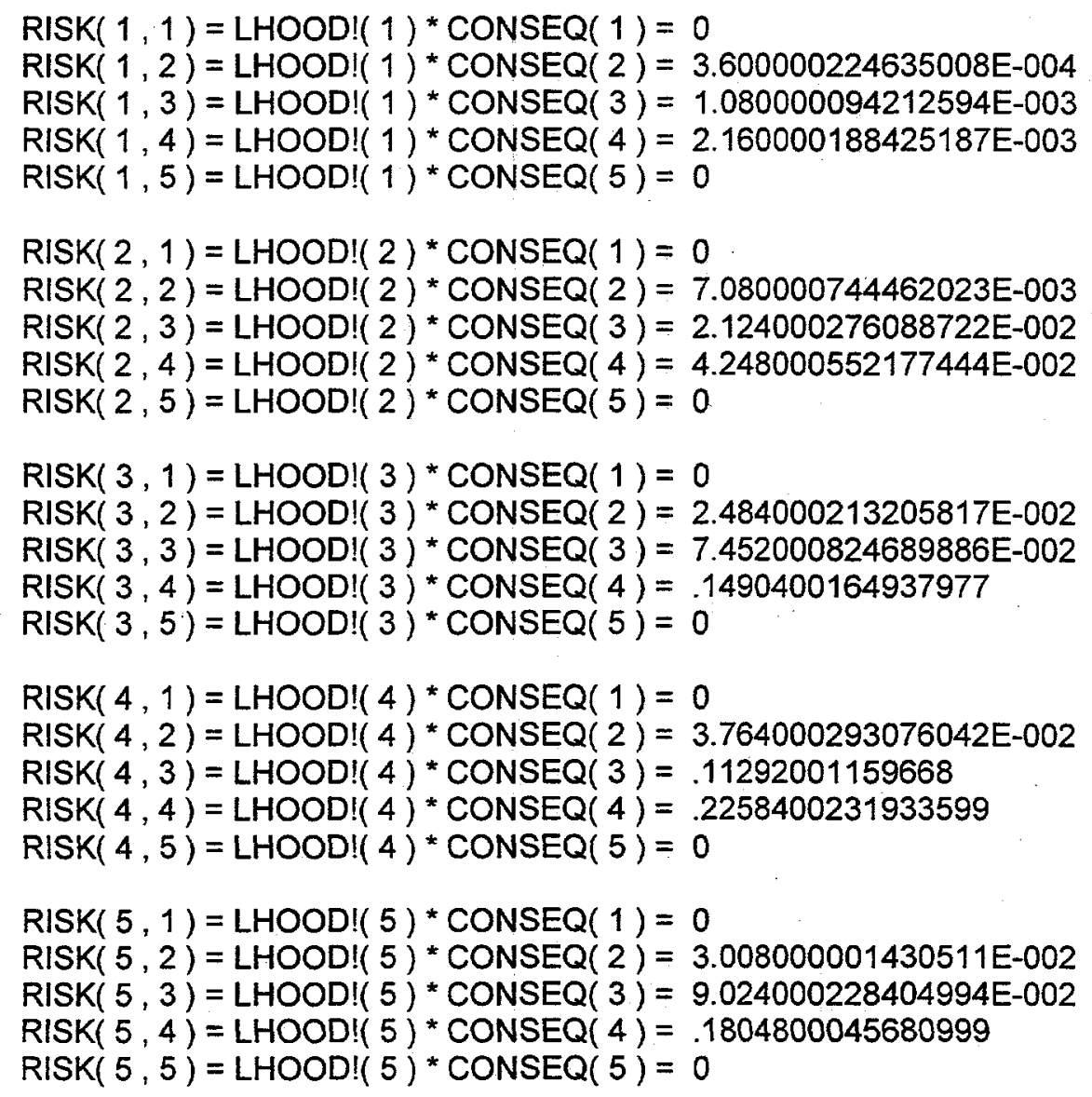


$\operatorname{RISKCAT}(1)=\operatorname{RISK}(1,1)+\operatorname{RISK}(1,2)+\operatorname{RISK}(1,3)+\operatorname{RISK}(2,1)+\operatorname{RISK}(3,1)+\operatorname{RISK}(4,1)+\operatorname{RISK}(5,1)$ $=1.440000138245523 \mathrm{E}-003$

$\operatorname{RISKCAT}(2)=\operatorname{RISK}(2,3)+\operatorname{RISK}(3,2)+\operatorname{RISK}(4,2)+\operatorname{RISK}(5,2)+\operatorname{RISK}(2,2)+\operatorname{RISK}(1,4)=$ .1230400055646896

$\operatorname{RISKCAT}(3)=\operatorname{RISK}(2,4)+\operatorname{RISK}(3,3)+\operatorname{RISK}(1,5)+\operatorname{RISK}(5,3)+\operatorname{RISK}(4,3)=.3201600313186646$

$\operatorname{RISKCAT}(4)=\operatorname{RISK}(2,5)+\operatorname{RISK}(3,5)+\operatorname{RISK}(3,4)+\operatorname{RISK}(4,4)+\operatorname{RISK}(5,4)=.5553600192070007$

$\operatorname{RISKCAT}(5)=\operatorname{RISK}(4,5)+\operatorname{RISK}(5,5)=0$

The final values of the Risk category probabilities are:

Probability for Risk category Negligible $=0.001$

Probability for Risk category Low $=0.123$

Probability for Risk category Medium $=0.320$

Probability for Risk category High $=0.555$

Probability for Risk category Very High $=0.000$ 



\section{APPENDIX G - Distribution Disk}

\section{Distribution Disk:}

\section{Contents:}

\begin{tabular}{|l|l|l|}
\hline \multicolumn{1}{|c|}{ FILE } & \multicolumn{1}{c|}{ FORMAT } & \multicolumn{1}{c|}{ Content } \\
\hline trace4.exe & Executable file & Trace Model \\
\hline It_eval4.exe & Executable file & Insider Theft \\
\hline ot_eval4.exe & Executable file & Outsider Theft \\
\hline oa_eval4.exe & Executable file & Outsider Attack \\
\hline st_eval4.exe & Executable file & State Transfer \\
\hline xp_eval.exe & Executable file & Transportation \\
\hline summary.xls & Excel & User summary / Table \\
\hline Presentation.ppt & PowerPoint & Summary - Project Overview \\
\hline
\end{tabular}

G.1 
PNNL-11993

\section{Distribution}

No. of

Copies

\section{OFFSITE}

10 E. K. Vaden

Office of Intelligence, $\mathbb{N}-1$

Forrestal Building

1000 Independence Avenue

Washington, DC 20585

4 D. Whitford (3)

R. O. Gilbert

Battelle Washington Operations

901 D Street

370 L'Enfant Promenade, S.W.

Suite 900

Washington, DC 20024-2115
No. of

Copies

\section{ONSITE}

15 Pacific Northwest National Laboratory

J. S. Dukelow (3)

K8-37

G. M. Gelston

K6-80

R. L. Gruel

K8-60

R. C. Hanlen

K6-40

W. A. Hesser

A0-21

J. E. Loftis

S7-41

D. A. Seaver

K8-03

C. E. Willingham

P8-50

Technical Report Files (5)

Distr. 1 